\title{
Palladium-mediated organofluorine chemistry
}

\author{
Ana C. Albéniz, Juan A. Casares \\ IU CINQUIMA/Química Inorgánica. Universidad de Valladolid. 47071-Valladolid. \\ Spain.
}

\section{Contents}

1. Introduction

2. C-C Coupling reactions of fluorinated reagents.

2.1. Overview of catalytic $\mathrm{C}-\mathrm{C}$ coupling reactions of fluorinated derivatives

2.1.1. C-C coupling reactions of fluorinated alkyls.

2.1.2. C-C coupling reactions of fluorinated aryl derivatives

2.1.3. $\mathrm{C}-\mathrm{C}$ coupling reactions of fluorinated arenes

2.1.4. C-C coupling reactions of fluorinated alkenyls

2.2. The $\mathrm{Pd}-\mathrm{R}_{\mathrm{F}}$ bond

2.2.1 Pd-Alkyl ${ }_{\mathrm{F}}$ bonds

2.2.2 $\mathrm{Pd}-\mathrm{Ar}_{\mathrm{F}}$ bonds

2.3. Elementary steps in organofluorine palladium catalyzed $\mathrm{C}-\mathrm{C}$ coupling processes

2.3.1. Oxidative addition and related processes

2.3.2. Transmetalation

2.3.3. Reductive Elimination

2.3.4. 2,1-Insertion

2.3.5. 1,1-Insertion (migratory insertion)

3. C-F activation and fluorination

3.1. Overview of catalytic $\mathrm{C}-\mathrm{C}$ and $\mathrm{C}-\mathrm{X}$ coupling reactions where a $\mathrm{C}-\mathrm{F}$ bond is cleaved.

3.1.1. C-C coupling of fluorinated aryls

3.1.2. C-C coupling of fluorinated alkenes

3.1.3. Allylic Substitutions of a fluorine atom.

3.1.4. Hydrodefluorination reactions.

3.2. Overview of catalytic $\mathrm{C}-\mathrm{F}$ forming reactions.

3.2.1. Pd-catalyzed fluorination with electrophilic fluorine sources.

3.2.2. Pd-catalyzed fluorination with nucleophilic fluorine sources.

3.3. The Pd-F bond

3.4. Oxidative Addition of the C-F bond

3.5. $\beta-\mathrm{F}$ elimination and $\alpha-\mathrm{F}$ elimination

3.6. Other activation routes for $\mathrm{C}-\mathrm{F}$ cleavage.

3.7. Reductive elimination of $\mathrm{R}-\mathrm{F}$

4. Conclusion

Acknowledgements

References 


\section{Introduction}

The substitution of fluorine for hydrogen in a molecule may result in profound changes in its properties and behaviour. Fluorine does not introduce special steric constraints since the $\mathrm{F}$ atom has a small size. However, the changes in bond polarity and the possibility of forming hydrogen bonds with other hydrogen donor fragments in the same or other molecules, may change the solubility and physical properties of the fluorinated compound when compared to the non-fluorinated one. Fluorine forms strong bonds to other elements and this ensures a good chemical stability. Altogether, fluorinated compounds are very attractive in materials chemistry and in medicinal chemistry, where many biologically active molecules and pharmaceuticals do contain fluorine in their structure and this has been shown to be essential for their activity. ${ }^{1,2}$

The synthesis of fluorinated compounds is a thriving field, driven by the huge interest of these molecules. A non-comprehensive list of recent reviews is given in references 3-19. ${ }^{1,3-19}$ Some of the challenges for the preparation of organofluorine derivatives lay on the development of suitable fluorine reagents that are not too reactive, as fluorine itself, or too unreactive because in order to give the desired reaction there is the need of the cleavage of a strong E-F bond. Some of the well developed and useful catalytic reactions for non-fluorinated compounds have been applied to the synthesis of fluoroderivatives but, in many cases, the reactions are not as effective as expected or simply do not work. In the last years, a strong impulse has been given to this field and, being palladium one of the most important metal in $\mathrm{C}-\mathrm{C}$ bond forming reactions, new catalytic processes have been developed using complexes of this metal. Other metallic catalysts have also been introduced in organofluorine syntesis, specially copper. ${ }^{3,10,13,18-}$ 
This chapter is intended to give an overview of the synthetic procedures of fluorinated molecules based on the use of palladium complexes, paying attention to the reported studies on the fundamental organometallic reactions that are involved as individual steps in those synthetic procedures. This is specially interesting since the understanding of these steps has allowed to explain the failure of some reactions and to develop new effective processes.

Long before the outburst of the catalytic synthesis of fluorinated compounds, the special stability of the organometallic palladium derivatives with fluorinated groups, specially fluoroaryls, had been recognized. Thus, a huge number of perfluoro and fluorohaloaryl complexes of palladium are known. ${ }^{21}$ Perfluoroalkyl derivatives have also been prepared. ${ }^{22}$ These fluorinated groups play the role of auxiliary ligands in many reactions but they have also allowed the isolation and study of unusual coordination modes of the aryl groups, ${ }^{23}$ unusual oxidation states for the metal, ${ }^{24}$ etc. The presence of a fluorinated group in the complexes is very convenient since ${ }^{19} \mathrm{~F}$ NMR spectroscopy, having high sensitivity and displaying a wide chemical shift range, can be used as structural tool. In this way the Pd-fluoroaryl moiety has been used as a reporter of the symmetry of the molecule and has allowed the study of a variety of complex fluxional processes, isomerization, and ligand substitution and exchange reactions, ${ }^{25}$ as well as the study of palladium-catalyzed polymerization reactions. ${ }^{26} \mathrm{~A}$ review covering the use of fluroraryl groups as reporter tools is available. ${ }^{27}$ Also, the use of ${ }^{19} \mathrm{~F}$ NMR has been very important in the study of other processes where the $\mathrm{Pd}-\mathrm{R}_{\mathrm{F}}$ bond is cleaved and these examples will be discussed here.

The contents of this chapter are organized in two main parts devoted, on one hand, to the formation of $\mathrm{C}-\mathrm{C}$ bonds where one of the coupled groups is a fluorinated one, and to those reactions that involve the cleavage or the formation of a C-F bond on 
the other. In each part a survey of the known palladium catalytic processes are given followed by the reported studies of individual organometallic reactions that play a role in the catalytic processes and give support to the studied or proposed mechanisms for them.

\section{C-C Coupling reactions of fluorinated reagents.}

\subsection{Overview of catalytic $\mathrm{C}-\mathrm{C}$ coupling reactions of fluorinated derivatives.}

The number of catalytic reactions that use fluorinated substituents is enormous. For example, it is common to find a fluorine atom or a $\mathrm{CF}_{3}$ group in an aromatic reagent to modulate the electronic properties of the aryl group. Most of these reactions are not different from other catalysis with non-fluorinated reagents. Thus, only a selection of fluorinated groups will be mentioned in this chapter, mainly polyfluorinated species or those examples where the fluorine atoms in any of the reagents is close to the substituted position and may introduce enough differences in the reaction rate or outcome. Efficient catalytic processes for these substrates have been developed with the aid of bulky phosphines, that have also proved to be very effective for the coupling of other reluctant groups such as alkyls. Figure 1 shows some of these ligands that will be mentioned in this chapter when discussing the coupling reactions they are involved in.

Fluorinated groups have been used as auxiliaries in catalytic $\mathrm{C}-\mathrm{C}$ reactions in such a way that they do not get incorporated into the final product. Among them, perfluoroalkyl sulfonates (mostly the so called nonaflates), ${ }^{28-30}$ and pentafluorobenzoyl oximes $^{31}$ as electrophilic reagents that undergo oxidative addition to $\operatorname{Pd}(0)$ in cross coupling reactions. This application will not be further discussed here. 
<smiles>[R]c1cc([R7])c(-c2ccccc2)c(P)c1</smiles>

$\begin{array}{lcccl}\mathrm{R}^{1} & \mathrm{R}^{2} & \mathrm{R}^{3} & \mathrm{R}^{4} & \text { name } \\ \text { OMe } & \mathrm{OMe} & \mathrm{H} & \mathrm{Cy} & \text { SPhos } \\ \text { Oi-Pr } & \mathrm{O} i-\mathrm{Pr} & \mathrm{H} & \mathrm{Cy} & \text { RuPhos } \\ i-\mathrm{Pr} & i-\mathrm{Pr} & i-\mathrm{Pr} & \mathrm{Cy} & \text { XPhos } \\ \mathrm{Cy} & \mathrm{Cy} & \mathrm{Cy} & \mathrm{t}-\mathrm{Bu} & \text { Cy-t-BuXPhos } \\ \mathrm{H} & \mathrm{H} & \mathrm{H} & t-\mathrm{Bu} & \text { JohnPhos }\end{array}$<smiles>[R7]c1cc(P)cc(P)c1-c1c(OC)ccc(OC)c1P</smiles>

$\begin{array}{ccccc}\mathrm{R}^{1} & \mathrm{R}^{2} & \mathrm{R}^{3} & \mathrm{R}^{4} & \text { name } \\ \mathrm{Pr} & i-\operatorname{Pr} & i-\operatorname{Pr} & \mathrm{Cy} & \text { BrettPhos } \\ \mathrm{Pr} & i-\operatorname{Pr} & i-\operatorname{Pr} & t \text {-Bu } & t \text {-BuBrettPhos }\end{array}$<smiles>CC1(C)c2cccc(P)c2OC2C(Pc3ccccc3)=CC=CC21</smiles>

Xantphos<smiles>Pc1ccccc1OC1CC=CC=C1Pc1ccccc1</smiles>

DPEPhos<smiles>Pc1c(-c2c(Pc3ccccc3)ccc3ccccc23)ccc2ccccc12</smiles>

(R)-binap<smiles>CC(C)[C@H]1COC(c2ccccc2Pc2ccccc2)=N1</smiles>

(S)-XPHOS

Figure 1. Bulky phosphine ligands used in Pd-catalyzed coupling reactions of organofluorides.

Palladium catalysis has also benefited from the introduction of fluoroalkyl chains in ligands or reaction partners to gain solubility of either the catalyst or the reagents in fluorous solvents or super-critical $\mathrm{CO}_{2}$. Some of these catalytic systems can be recyclable. Fluorinated surfactants or stabilizers of nanoparticles have also been reported. Both uses of fluoroorganyls will not be discussed in this chapter.

The reactions gathered in this section are ordered according to the type of fluorinated group that participates in the coupling reaction. In each of them, subsections labeled with the different type of processes can be found. 


\subsubsection{C-C coupling of fluorinated alkyl derivatives}

The palladium-catalyzed fluoroalkylation of organic derivatives is not an easy task. Two main problems arise for the application of the well known Pd-catalyzed C-C coupling reactions to these groups: i) the availability of suitable fluoroalkyl transmetalating reagents and ii) the sluggish reductive elimination of perfluoroalkyl palladium derivatives, so other reaction pathways can compete to give undesired byproducts. The advances in this field have concentrated in solving these two problems, specially problematic for the simplest member of the fluoroalkyls, the $\mathrm{CF}_{3}$ group. Thus, the functionalization with the trifluoromethyl group relies on the use of new silicon or copper trifluoromethyl reagents. The bulky phosphines collected in Figure 1, which ensure low coordination numbers and bidentate binding modes when required, have overcome the reductive elimination problem.

Some palladium-catalyzed processes are used to transform substrates that already bear the fluorinated group such as the enantioselective hydrogenation of fluorinated imines. ${ }^{32}$ However the examples collected here refer to Pd-mediated processes that involve the introduction of a fluorinated group in the molecule mainly, but not exclusively, with the formation of a new C-C bond.

\section{Radical reactions}

Perfluoroalkyl halides are well known sources of perfluoroakyl radicals. These are kinetically more stable than non-fluorinated alkyl radicals due to the limited number of termination processes which affect them. ${ }^{1}$ Radical mechanisms are often invoked in palladium catalyzed perfluoroalkylation reactions, and in fact most of the early metal catalyzed processes involving perfluoroalkyl reagents were classified as radical 
reactions. The radical initiation reaction can be promoted by irradiation, heating, use of radical initiators, and also by metals. ${ }^{1}$

In 1987 Huang and Zhou reported the formation of perfluoroalkyldialkylamines and perfluoroalkylalkylenamines in the reaction of trialkylamines with perfluoroalkyl iodides catalyzed by triphenylphosphine complexes of palladium, platinum, or nickel (Scheme 1). ${ }^{33}$ The reaction is proposed to follow a radical pathway in which a palladium(0) complex act as radical initiator.

$$
\begin{aligned}
& 2 \mathrm{R}_{\mathrm{F}} \mathrm{CF}_{2} \mathrm{I}+3 \mathrm{RCH}_{2} \mathrm{CH}_{2} \mathrm{NR}^{1} \mathrm{R}^{2} \stackrel{\left[\mathrm{M}\left(\mathrm{PPh}_{3}\right)_{4}\right]}{\longrightarrow} \mathrm{R}_{\mathrm{F}} \mathrm{CF}_{2} \mathrm{CR}=\mathrm{CHNR}^{1} \mathrm{R}^{2}+\mathrm{R}_{\mathrm{F}} \mathrm{CF}_{2} \mathrm{H} \\
& \mathrm{M}=\mathrm{Ni}, \mathrm{Pd}, \mathrm{Pt} \\
& +2\left[\mathrm{RCH}_{2} \mathrm{CH}_{2} \mathrm{NHR}_{1}{ }_{2}\right]_{l} \\
& \mathrm{R}_{\mathrm{F}} \mathrm{CF}_{2} \mathrm{l}+\left[\mathrm{Pd}^{0}\right] \longrightarrow \mathrm{R}_{\mathrm{F}} \mathrm{CF}_{2} \cdot+[\mathrm{Pdl} \cdot] \\
& \mathrm{R}_{\mathrm{F}} \mathrm{CF}_{2}+\mathrm{RCH}_{2} \mathrm{CH}_{2} \mathrm{NR}^{1} \mathrm{R}^{2} \longrightarrow \mathrm{R}_{\mathrm{F}} \mathrm{CF}_{2}^{-}+\mathrm{RCH}_{2} \mathrm{CH}_{2} \stackrel{\oplus}{\mathrm{NR}^{1} \mathrm{R}^{2}}
\end{aligned}
$$

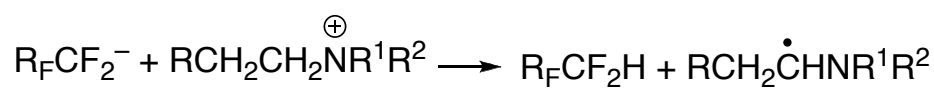

$$
\begin{aligned}
& \mathrm{RCH}_{2} \dot{\mathrm{C}} \mathrm{HNR}^{1} \mathrm{R}^{2}+[\mathrm{Pdl} \cdot] \longrightarrow \mathrm{RCH}=\mathrm{CHNR}^{1} \mathrm{R}^{2}+[\mathrm{HPdl}] \\
& {[\mathrm{HPdl}]+\mathrm{RCH}_{2} \mathrm{CH}_{2} \mathrm{NR}^{1} \mathrm{R}^{2} \longrightarrow\left[\mathrm{RCH}_{2} \mathrm{CH}_{2} \mathrm{NHR}^{1} \mathrm{R}^{2}\right] \mathrm{I}+\left[\mathrm{Pd}^{0}\right]}
\end{aligned}
$$

Scheme 1

A year before, Ishihara et al. had reported that the addition of fluoroalkyl iodides to olefins and alkynes is efficiently catalyzed by $\left[\mathrm{Pd}\left(\mathrm{PPh}_{3}\right)_{4}\right]$, and that radical scavengers retarded the reaction. ${ }^{34}$ Qiu and Burton extended the scope of the reaction to the use of alkene,1,2-diols and they found that the same reaction can be performed by using by peroxides as radical initiators supporting a radical mechanism (Scheme 2). ${ }^{35}$

$$
\begin{aligned}
\mathrm{R}_{\mathrm{F}} \mathrm{l}+\mathrm{CH}_{2}=\mathrm{CHR} \stackrel{\text { Cat. }}{\longrightarrow} & \mathrm{R}_{\mathrm{F}} \mathrm{CH}_{2} \mathrm{CHIR} \\
& \text { Cat. }=\left[\mathrm{Pd}\left(\mathrm{PPh}_{3}\right)_{4}\right] \text { or }\left(\mathrm{PhCO}_{2}\right)_{2}
\end{aligned}
$$

Scheme 2 
In the presence of a catalytic amount of $\left[\mathrm{Pd}\left(\mathrm{PPh}_{3}\right)_{4}\right]$, iododifluoromethyl alkyl and phenyl ketones react with alkenes to give the corresponding $\alpha, \alpha$-difluoro- $\gamma$ iodoketones (Scheme 3). ${ }^{36}$ The reaction can be completely suppressed by a radical inhibitors such, di-tert-butyl nitroxide or hydroquinone. A radical mechanism initiated by the palladium assisted homolytic breaking of the $\mathrm{C}-\mathrm{I}$ bond is proposed.

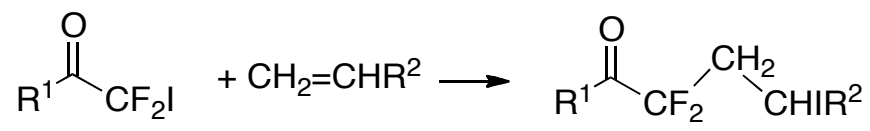

Scheme 3

Motoda et al. have shown that the radical addition takes place under very mild conditions by the use of a heterogeneous aqueous system, without the use of hydrophilic co-solvents or phase transfer catalysts (Scheme 4). ${ }^{37}$ The reaction takes place in two hours at room temperature.

$$
\begin{aligned}
& \mathrm{R}^{1}=n-\mathrm{C}_{10} \mathrm{H}_{21}, \mathrm{AcO}\left(\mathrm{CH}_{2}\right)_{3}, \mathrm{CH}_{3} \mathrm{CO}\left(\mathrm{CH}_{2}\right)_{3} \\
& \mathrm{~L}=\mathrm{dppf} \\
& \mathrm{G}=\mathrm{O},\left(\mathrm{MeOCH}_{2}\right)_{2} \mathrm{C}, \mathrm{BnN}
\end{aligned}
$$

Scheme 4

Looking for a wider scope of the synthetic procedures, cross-coupling methodologies have been applied to perfluoroalkyl groups. The fluorinated group can be introduced in the oxidative addition step (typically as $\mathrm{R}_{\mathrm{F}}-\mathrm{X} \mathrm{X}=\mathrm{I}$, TfO, etc.) or as 
nucleophile through a transmetallation reaction. Examples of the different cross coupling reactions applied to fluoroalkyls are given below.

Perfluoroalkyl groups in the Stille reaction

Matsubara et. al. succeeded in the cross coupling of alkynyl, alkenyl and allyl tributyltin derivatives with perfluoroalkyl iodides such $\mathrm{CF}_{3} \mathrm{I}, n-\mathrm{C}_{4} \mathrm{~F}_{9} \mathrm{I}$ or $\mathrm{C}_{6} \mathrm{~F}_{13} \mathrm{I}$, using $\left[\mathrm{Pd}\left(\mathrm{PPh}_{3}\right)_{4}\right](10 \%)$ as catalyst (Scheme 5). ${ }^{38}$ The reaction was performed in hexane at 70 ${ }^{\circ} \mathrm{C}$. No details about the mechanism were provided.

$$
\mathrm{R}_{\mathrm{F}} \mathrm{I}+\mathrm{R}^{2} \mathrm{SnBu}_{3} \stackrel{\left[\mathrm{Pd}\left(\mathrm{PPh}_{3}\right)_{4}\right]}{\longrightarrow} \mathrm{R}_{\mathrm{F}}-\mathrm{R}^{2}+\mathrm{ISnBu}_{3}
$$

Scheme 5

The cross-coupling of $\beta$-perfluoroalkyl-substituted alkyl halides with organostannanes such as allyl, arylethynyl, and aryltributylin is efficiently catalyzed by $\left[\mathrm{PdCl}_{2}\left(\mathrm{PPh}_{3}\right)_{2}\right]$ and by $\left[\mathrm{Pd}\left(\mathrm{PPh}_{3}\right)_{4}\right]$. When the reaction is carried out under $\mathrm{CO}$ pressure, carbonylative coupling of the halides took place affording the corresponding fluorinecontaining ketones in good yields (Scheme 6). ${ }^{39}$

$$
\begin{aligned}
\mathrm{R}_{\mathrm{F}} \mathrm{CH}_{2} I+\mathrm{R}^{2} \mathrm{SnBu}_{3} \stackrel{[\mathrm{Pd}]}{\longrightarrow} \mathrm{R}_{\mathrm{F}} \mathrm{CH}_{2}-\mathrm{R}^{2}+\mathrm{ISnBu}_{3} \\
\mathrm{R}_{\mathrm{F}} \mathrm{CH}_{2} \mathrm{I}+\mathrm{R}^{2} \mathrm{SnBu}_{3} \stackrel{[\mathrm{Pd}]}{\mathrm{CO}} \mathrm{R}_{\mathrm{F}}+\mathrm{ISnBu}_{3} \\
{[\mathrm{Pd}]=\left[\mathrm{Pd}\left(\mathrm{PPh}_{3}\right)_{4}\right] ;\left[\mathrm{PdCl}_{2}\left(\mathrm{PPh}_{3}\right)\right] } \\
\mathrm{R}^{2}=\text { phenyl, phenylethynyl, phenylehtenyl, allyl } \\
\mathrm{R}_{\mathrm{F}}=\mathrm{CF}_{3}, \mathrm{C}_{3} \mathrm{~F}_{7}, \mathrm{C}_{6} \mathrm{~F}_{13}, \mathrm{C}_{8} \mathrm{~F}_{17}
\end{aligned}
$$

Scheme 6 
The synthesis of group 15 and 16 perfluoroalkyl derivatives can be carried out by the palladium catalyzed cross coupling of perfluoroalkyliodides with organotin compounds such as $\mathrm{Bu}_{3} \mathrm{SnPPh}_{2}, \mathrm{Bu}_{3} \mathrm{SnAsPh}_{2}, \mathrm{Bu}_{3} \mathrm{SnSePh}$. The formation of perfluoroalkylphosphine oxides (the coupling reaction was followed by the oxidation of the phosphine), perfluoroalkylarsine or perfluoroalkyl selenide took place. ${ }^{40,41}$ The best results were obtained by using $\left[\mathrm{PdCl}_{2}\left(\mathrm{PPh}_{3}\right)_{2}\right]$ as catalyst with a small excess of $\mathrm{PPh}_{3}$ and $\mathrm{CsF}$ as aditive (Scheme 7). The use of other palladium sources (such $\left[\mathrm{Pd}\left(\mathrm{PPh}_{3}\right)_{4}\right]$ or $\left.\mathrm{Pd}_{2}(\mathrm{dba})_{3}\right)$, or other phosphines as ligands led to worse results. ${ }^{42,43}$

$$
\begin{aligned}
& \mathrm{R}_{\mathrm{F}} \mathrm{I}+\mathrm{R}_{2} \mathrm{P}-\mathrm{SnBu}_{3} \stackrel{[\mathrm{Pd}]}{\mathrm{CsF}, \mathrm{L}} \stackrel{\text { air }}{\longrightarrow} \mathrm{R}_{\mathrm{F}} \mathrm{P}(\mathrm{O}) \mathrm{R}_{2}+\mathrm{ISnBu}_{3} \\
& \mathrm{R}_{\mathrm{F}} \mathrm{I}+\mathrm{R}_{\mathrm{n}} \mathrm{E}-\mathrm{SnBu}_{3} \stackrel{[\mathrm{Pd}]}{\mathrm{CsF}, \mathrm{L}} \longrightarrow \mathrm{R}_{\mathrm{F}}-\mathrm{ER}_{\mathrm{n}}+\mathrm{ISnBu}_{3} \\
& {[\mathrm{Pd}]=\left[\mathrm{Pd}\left(\mathrm{PPh}_{3}\right)_{4}\right],\left[\mathrm{PdCl}_{2}\left(\mathrm{PPh}_{3}\right)_{2}\right],\left[\mathrm{Pd}_{2}(\mathrm{dba})_{3}\right],\left[\mathrm{Pd}_{2}(\mu-\mathrm{Cl})_{2}(\text { allyl })_{2}\right]} \\
& \mathrm{L}=\mathrm{PPh}_{3} \mathrm{P}(\mathrm{o}-\mathrm{tol})_{3}, \mathrm{PCy}_{3} \\
& \mathrm{ER}_{\mathrm{n}}=\mathrm{SePh}, \mathrm{AsPh}_{2}
\end{aligned}
$$

Scheme 7

Introduction of fluorinated groups using the Negishi reaction

The Negishi cross-coupling between perfluoroalkylzinc iodides (including $\mathrm{CF}_{3}$, $i-\mathrm{C}_{3} \mathrm{~F}_{7}, n-\mathrm{C}_{3} \mathrm{~F}_{7}$, and $\left.n-\mathrm{C}_{4} \mathrm{~F}_{9}\right)$ and allyl or vinyl bromides, or aryl iodides has been described. ${ }^{44,45}$ The reactions were performed one pot reacting the perfluoroalkyl iodides with ultrasonically dispersed zinc in the presence of the palladium catalyst and the aryl, vinyl or allyl halide (Scheme 8). In a previous work, Kitazume et al. had shown that under this conditions the organozinc $\mathrm{ZnIR}_{\mathrm{F}}$ is formed. ${ }^{46} \mathrm{Pd}(\mathrm{AcO})_{2},\left[\mathrm{PdCl}_{2}\left(\mathrm{PPh}_{3}\right)_{2}\right]$ and $\left[\mathrm{Pd}\left(\mathrm{PPh}_{3}\right)_{4}\right]$ were used as catalyst, and no difficulties derived from the reductive elimination step were reported. 


$$
\begin{aligned}
& \mathrm{R}_{\mathrm{F}} \mathrm{I}+\mathrm{R}^{2}-\mathrm{X} \underset{[\mathrm{Pd}]}{\stackrel{\mathrm{Zn} / \mathrm{THF}}{\longrightarrow}} \mathrm{R}_{\mathrm{F}}-\mathrm{R}^{2} \\
& {[\mathrm{Pd}]=\left[\mathrm{Pd}\left(\mathrm{PPh}_{3}\right)_{4}\right],\left[\mathrm{PdCl}_{2}\left(\mathrm{PPh}_{3}\right)_{2}\right],\left[\mathrm{Pd}(\mathrm{OAc})_{2}\right],\left[\mathrm{Pd}_{2}(\mu-\mathrm{Cl})_{2}(\text { allyl })_{2}\right]} \\
& \mathrm{R}_{\mathrm{F}}=\mathrm{CF}_{3}, \mathrm{C}_{2} \mathrm{~F}_{5}, n-\mathrm{C}_{3} \mathrm{~F}_{7}, i-\mathrm{C}_{3} \mathrm{~F}_{7}, n-\mathrm{C}_{4} \mathrm{~F}_{9} . \\
& \mathrm{R}^{2}=\text { aryl, vynyl, styryl, phenylallyl. }
\end{aligned}
$$

Scheme 8

Also based on the Negishi cross-coupling methodology, the synthesis of 2perfluoroalkylpirrole by the reaction of the organozinc with perfluoroalkyliodide has been reported. $^{47}$

\section{Introduction of fluorinated groups using the Suzuki reaction}

Suzuki-Miyaura protocols have been used to synthesize different types of pefluoroalkylated molecules. For example, trifluoroacetyl and trifluoroacetimidoyl moieties can be successfully introduced to aromatic compounds in this way (Scheme 9). ${ }^{48}$

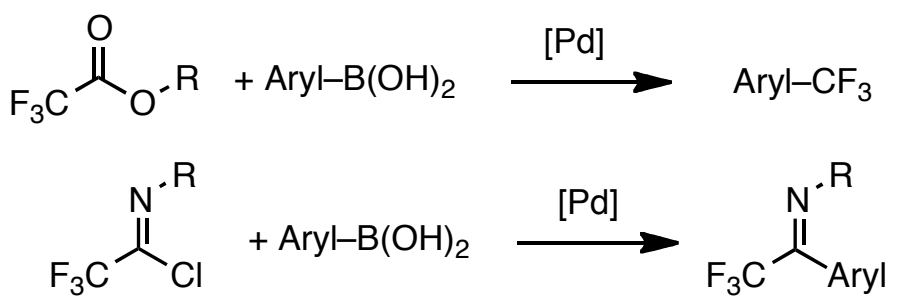

Scheme 9

The Suzuki reaction has been applied to $\beta$-fluorinated alkyl derivatives as organic electrophiles. Liang et. al. have reported the cross coupling of $\mathrm{CF}_{3} \mathrm{CH}_{2} \mathrm{I}$ wih heteroarylboronic esthers. ${ }^{49}$ The use of bulky dialkylbiphenylphosphines, particularly XPhos, allow the cross coupling of pyridyl pinacol boronate and other aryl boronates in moderate yields $(28 \%-60 \%)$. Other fluoroalkyl iodides such $\mathrm{CF}_{3} \mathrm{CF}_{2} \mathrm{CH}_{2} \mathrm{I}$ gave poorer yields (about $20 \%$ ). The reaction proceeds in DMF as solvent and requires the use of additives such $\mathrm{CsF}$, water, and $\mathrm{CuCl}$ (Scheme 10). The role of the $\mathrm{CuCl}$ is unclear: 
Although the copper catalyzed cross-coupling of alkyl-halides and arylboronic acids has been described, ${ }^{50}$ Liang et al. reported that the use of $\mathrm{CuCl}$, without palladium, as catalyst yielded only traces of the cross coupling product.

$$
\begin{aligned}
& \mathrm{CF}_{3} \mathrm{CH}_{2} \mathrm{l}+\mathrm{R}^{2}-\mathrm{B}(\mathrm{OR})_{2} \underset{\text { "aditives" }}{\stackrel{[\mathrm{Pd}]}{\longrightarrow}} \mathrm{R}_{\mathrm{F}} \mathrm{CH}_{2}-\mathrm{R}^{2} \\
& \mathrm{R}^{2}=\text { aryl or heteroaryl ; } \mathrm{B}(\mathrm{OR})_{2}=\mathrm{B}(\mathrm{OH})_{2} \text { or } \mathrm{B}(\text { pinacolate }) \\
& {[\mathrm{Pd}]=\left[\mathrm{Pd}_{2}(\mathrm{dba})_{3}\right] \cdot \mathrm{CHCl}_{3}+\mathrm{XPhos} ; \text { "aditives": CuCl, CsF, } \mathrm{H}_{2} \mathrm{O}}
\end{aligned}
$$

Scheme 10

So far, the best results in the Suzuki cross-coupling of trifluoroethyl derivatives have been reported by Zhao and $\mathrm{Hu} .{ }^{51}$ The use of $\mathrm{Pd}_{2}(\mathrm{dba})_{3} \cdot \mathrm{CHCl}_{3}$ and a large excess of Xantphos as catalytic system allow the synthesis or trifluoroethyl arenes with yields up to $92 \%$. In this system $\mathrm{Cs}_{2} \mathrm{CO}_{3}$ was used as base and a mixture of dioxane/water was used as solvent.

Not only trifluoroethyl iodide, but also trifluoroethyl tosylate undergo the Suzuki cross coupling. ${ }^{52}$ The reaction takes place under harsh conditions ( $4 \mathrm{~h}$ at $150^{\circ} \mathrm{C}$ in DMSO as solvent). The best catalytic system for this reaction involves two different palladium catalysts: one palladacycle complex and $\mathrm{Pd}(\mathrm{AcO})_{2}$ (Scheme 11). No explanation of the role of these two catalysts is reported, but the absence of any of them has a great effect on the yield, which is always moderate (25-60\%). Several "additives" have also to be added: $\mathrm{NaI}$ (up to 3 equivalents) base (3 equivalents) and extra ligand $\left(\mathrm{PPh}_{3}\right.$ or other phosphine). 


$$
\begin{aligned}
& \mathrm{CF}_{3} \mathrm{CH}_{2} \mathrm{I}+\mathrm{R}^{2}-\mathrm{B}(\mathrm{OH})_{2} \underset{\text { "aditives" }}{\stackrel{[\mathrm{Pd}]}{\longrightarrow}} \mathrm{R}_{\mathrm{F}} \mathrm{CH}_{2}-\mathrm{R}^{2} \\
& \text { aditives: } \mathrm{PPh}_{3}, \mathrm{~K}_{3} \mathrm{PO}_{4}, \mathrm{Nal}
\end{aligned}
$$

Scheme 11

As it is the case for trifluoromethylation, most successful metal catalyzed procedures to introduce the difluoromethyl group have focused in the use of copper as catalyst. $^{53}$ Palladium catalyzed reactions are a rarity. Nonetheless, the palladiumcatalyzed difluoroalkylation of aryl boronic acids containing functional groups (ester, thio- ether, trimethylsilyl, etc.) with commercially available bromodifluoromethylphosphonate, and with bromodifluoroacetate has been reported by Feng et al. ${ }^{54}$ The catalytic system includes the use of $\left[\mathrm{Pd}\left(\mathrm{PPh}_{3}\right)_{4}\right]$ or $\left[\mathrm{PdCl}_{2}\left(\mathrm{PPh}_{3}\right)_{2}\right]$ as palladium source, and Xantphos (2 to 4 equivalents respect to the palladium catalyst) as auxiliar ligand (Scheme 12). The role of Xantphos is not clear but, although different palladium sources could be used, the addition of Xanphos was critical for the catalysis. In the cross coupling reaction of bromodifluoroacetate the use of $\mathrm{CuI}$ as "co-promoter" was also required. The reaction has been used for the late-stage synthesis of biologically active compounds.

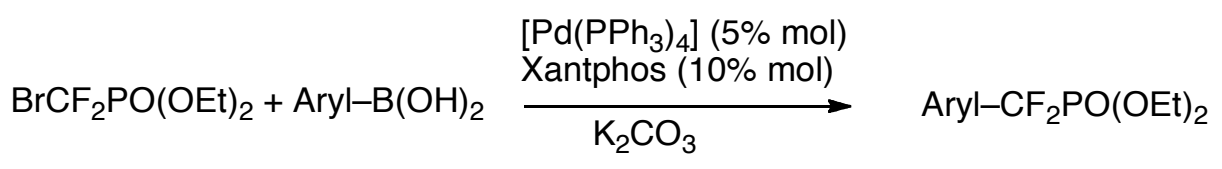

Scheme 12 
Zhang's group has also reported the use of the Suzuki-Miyaura methodology for the cross coupling of 3-bromo-3,3-difluoropropene with a variety of aryl boronates and arylboronic acids to produce the gem-difluoroallylated arenes. ${ }^{55}$ The reaction occurs in very high yields with low catalyst loading $\left(0.8 \%-0.01 \%\right.$ of $\mathrm{Pd}_{2}\left(\mathrm{dba}_{3}\right)$ or $\left.\left[\mathrm{Pd}\left(\mathrm{PPh}_{3}\right)_{4}\right]\right)$. The procedure has been scaled up to obtain $10 \mathrm{~g}$ of product, and applied to the synthesis of bioactive molecules (Scheme 13)

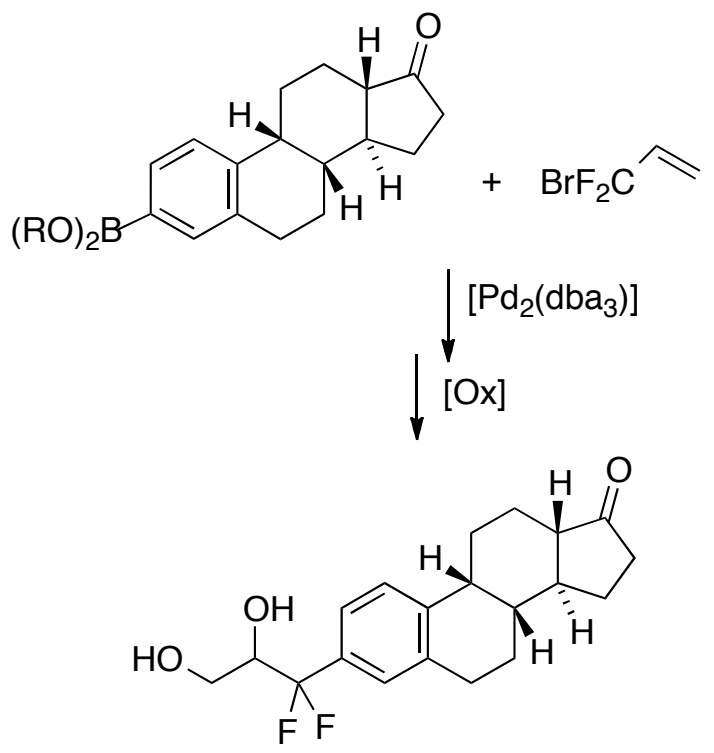

Scheme 13

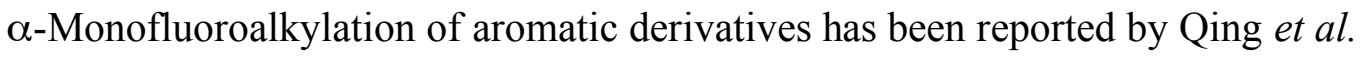
using the palladium-catalyzed Suzuki-Miyaura cross-coupling reactions of ethyl $\alpha$ bromo- $\alpha$-fluoroacetate with various structurally diverse arylboronic acids using a phosphine ligand, to afford $\alpha$-aryl- $\alpha$-fluoroacetates in moderate to good yields. ${ }^{56}$ The reaction also produces the hydrogenation of the bromofluoroacetate (Scheme 14), although this side-reaction may be minimized by using $\mathrm{PPh}_{3}$ as ligand. 


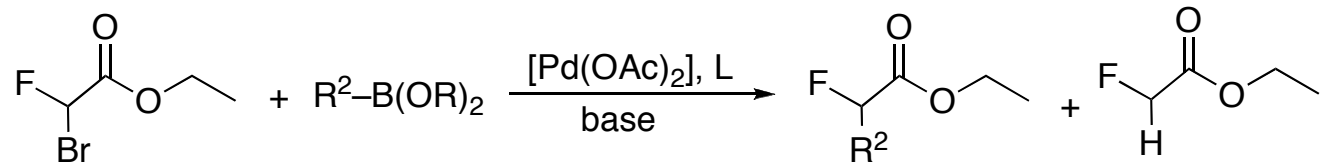

$\mathrm{R}_{2}=$ Aryl; $\mathrm{L}=$ biphenyldialkylphosphine, $\mathrm{PPh}_{3}$, dppe, dppb

base $=\mathrm{K}_{3} \mathrm{PO}_{4}, \mathrm{NaOAc}, \mathrm{Cs}_{2} \mathrm{CO}_{3}, \mathrm{CsF}$

Scheme 14

Use of fluoroalkyl silanes as fluoroalkylating reagents

Fluoroalkyl silyl derivatives combined with fluoride salts, have proved to be useful fluoroalkylating reagents in palladium catalysis. The most prominent compound of this class is trifluoromethyltrimethylsilane, also known as the Ruppert's reagent, which has been widely used to generate $\mathrm{CF}_{3}^{-}$in solution. ${ }^{8,57,58}$ Trifluoromethyltriethylsilane is commercially available, it is increasingly used and its applications have been reviewed. ${ }^{4,58,59}$ The reagent requires the use of a nucleophile (typically $\mathrm{F}^{-}$) to activate the $\mathrm{CF}_{3}-\mathrm{Si}$ bond. The $\mathrm{CF}_{3}^{-}$that is generated readily decomposes to difluorocarbene $\left(\mathrm{F}_{2} \mathrm{C}\right.$ : $)$ and fluoride $\left(\mathrm{F}^{-}\right)$, so the reaction rate of the reaction of this anion with the final reagent (the palladium complex) has to be high enough to avoid its accumulation in solution and its eventual decomposition. The main difficulty for the application of these reagents to palladium catalyzed reactions is the sluggish reductive elimination of perfluoroalkyl palladium derivatives, that makes possible the competition of other reaction pathways leading to unwanted products. This difficulty has been overcome by Buchwald et al. by using very bulky biphenyldialkylphosphines, such Bretphos or RuPhos (Scheme 15). With these ligands they succeeded in producing the palladium catalyzed cross coupling of arylchlorides with $\mathrm{CF}_{3} \mathrm{SiMe}_{3}$ or $\mathrm{CF}_{3} \mathrm{SiEt}_{3}$ in the presence of $\mathrm{CsF}$ at $120-140{ }^{\circ} \mathrm{C}$ in dioxane. ${ }^{60}$ The system shows a high tolerance for functional groups on the aryl moiety. The transmetalation of the $\mathrm{CF}_{3}$ group from $\mathrm{CF}_{3} \mathrm{SiEt}_{3}$ to palladium occurs at room temperature, allowing the isolation of the reaction intermediate $\left[\operatorname{PdAr}\left(\mathrm{CF}_{3}\right)(\right.$ BrettPhos $\left.)\right]$. 
The X-Ray structure of this complex shows the coordination of the BrettPhos ligand in a chelating mode, through the phosphorus and one of the oxygen atoms (Scheme 15). The same coordination mode is possible for the other ligands that are able to promote the reductive elimination: Ruphos and Xantphos.

$$
\begin{aligned}
& \mathrm{CF}_{3} \mathrm{SiEt}_{3}+\mathrm{R}^{2}-\mathrm{Cl} \underset{\mathrm{KF} \text {, dioxane }}{\stackrel{[\mathrm{Pd}], \mathrm{L}}{\longrightarrow}} \mathrm{CF}_{3}-\mathrm{R}^{2} \\
& \mathrm{R}^{2}=\text { aryl or heteroaryl } \\
& {[\mathrm{Pd}]=\mathrm{Pd}_{2}(\mathrm{dba})_{2} ;\left[\mathrm{Pd}_{2}(\mu-\mathrm{Cl})_{2}(\text { allyl })_{2}\right]} \\
& \mathrm{L}: \text { BrettPhos, RuPhos }
\end{aligned}
$$

Scheme 15

This complex undergoes a reductive elimination reaction in the presence of a different aryl chloride at the same rate and with identical yield of the trifluoromethylated aryl than that observed without the additional aryl chloride (Scheme 16). This supports the classical catalytic cycle for cross-coupling reactions in which only $\operatorname{Pd}(\mathrm{II})$ and $\operatorname{Pd}(0)$ species are involved in the catalytic cycle, ruling out the involvement of $\mathrm{Pd}(\mathrm{IV})$ complexes.

$$
\begin{aligned}
{\left[\mathrm{PdAr}^{1} \mathrm{CF}_{3}(\text { BrettPhos })\right] } & \stackrel{\text { Dioxane }}{80^{\circ} \mathrm{C}} \mathrm{Ar}^{1}-\mathrm{CF}_{3} \\
{\left[\mathrm{PdAr}^{1} \mathrm{CF}_{3}(\text { BrettPhos })\right]+\mathrm{Ar}^{2} \mathrm{Cl} } & \stackrel{\text { Dioxane }}{\stackrel{80^{\circ} \mathrm{C}}{\longrightarrow}}\left[\mathrm{PdAr}^{2} \mathrm{Cl}(\text { BrettPhos })\right]+\mathrm{Ar}^{1}-\mathrm{CF}_{3}
\end{aligned}
$$

Scheme 16

In 2011 Cho and Buchwald extended this procedure to the catalytic trifluoromethylation of cyclic vinyl triflates or nonaflates using $\mathrm{CF}_{3} \mathrm{SiMe}_{3}$ in the presence of $\mathrm{KF}$ or $\mathrm{CF}_{3} \mathrm{SiEt}_{3}$ with $\mathrm{RbF}$ as trifluoromethylating mixtures in a Hiyama-like cross-coupling reaction. ${ }^{61}$ The success of the reaction depends critically on the ligand 
used. For these reagents BrettPhos and ${ }^{\mathrm{t}} \mathrm{BuBrettPhos}$ gave poor results but the reaction performed well using ${ }^{t} \mathrm{BuXPhos}$ or $\mathrm{Cy}^{\mathrm{t}} \mathrm{BuXPhos}$. Cyclohexenyl triflates or nonaflates as electrophiles gave the best results. Five or seven membered alkenyl rings have either lower reactivity or undergo side-reactions that precluded the formation of the desired products in high yields.

\section{Other fluoroalkylating reagents: fluoroalkyl copper}

Although there are several methods for the synthesis of trifluoromethylcopper(I) complexes, ${ }^{62,20}$ the isolated complexes are not generally used as trifluoromethylating reagents in palladium catalyzed processes. This is probably related with the fact that, even in low concentration, the trifluoromethylcopper derivatives induce polytrifluoromethylation of palladium complexes and lead to inert derivatives of generic formula $\left[\mathrm{Pd}\left(\mathrm{CF}_{3}\right)_{\mathrm{n}} \mathrm{L}\right]^{(\mathrm{n}-2)}$. However, they are useful when generated in situ during the reaction using an equimolar mixture of $\mathrm{FSO}_{2} \mathrm{CF}_{2} \mathrm{COOMe}$ and $\mathrm{CuI}$. The use of readily available reagents derived from tetrafluoroethane $\beta$-sultone, which includes fluorosulfonyldifluoroacetic acid derivatives such as $\mathrm{FSO}_{2} \mathrm{CF}_{2} \mathrm{COOH}$ or $\mathrm{FSO}_{2} \mathrm{CF}_{2} \mathrm{COOMe}$ as trifluoromethylating and difluoromethylating reagents, has been recently revised (Scheme 17). ${ }^{11}$

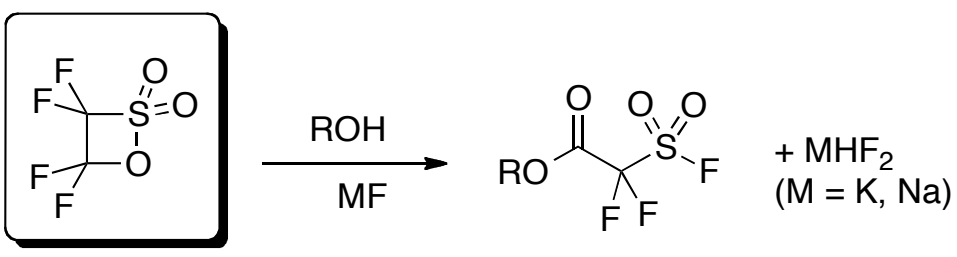

Tetrafluoroethane $\beta$-sultone

Scheme 17

Chen and $\mathrm{Wu}$ have found that $\mathrm{FSO}_{2} \mathrm{CF}_{2} \mathrm{COOMe}$ readily eliminates $\mathrm{CO}_{2}$ and $\mathrm{SO}_{2}$ in the presence of $\mathrm{CuI}$ in DMF at $60-80{ }^{\circ} \mathrm{C}$ to produce $\mathrm{CuCF}_{3}$ species. ${ }^{63}$ By using this 
system, Roche and Dolbier reported the aromatic trifluoromethylation of octafluoro[2.2]paracyclophanes, using $\mathrm{PdCl}_{2}$ as palladium source (Scheme 18). ${ }^{64}$ This substrate was particularly difficult to trifluoromethylate under standard coppercatalyzed trifluoromethylation and, in fact, the reaction takes place without palladium chloride but in very poor yields $(<20 \%)$. In the following year, Dolbier extended the use of this reaction to the trifluoromethylation of several octafluoro[2.2]paracyclophanes diiodides, furnishing the desired products in high yields. $^{65}$<smiles>COC(=O)OOS(=O)(=O)C(F)(F)c1ccc(C(F)(F)F)cc1</smiles>

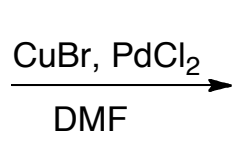<smiles>FC(F)(F)c1ccc(C(F)(F)F)c(C(F)(F)F)c1</smiles>

Scheme 18

This procedure has also been employed in the palladium catalyzed trifluoromethylation of porphyrins. ${ }^{66,67}$ Since porphyrins efficiently coordinate copper(I), [Cu(TPPBr)] (TPP: meso-tetraphenylporphyrin) were used as substrate for the catalysis to avoid the capture of $\mathrm{CuI}$ by the substrate (Figure 2). The reaction takes place in $\mathrm{DMF}$ at $100{ }^{\circ} \mathrm{C}$, being the combination of $\mathrm{Pd}_{2}(\mathrm{dba})_{3}$ and $\mathrm{AsPh}_{3}$ as ligand the best system for the catalysis, while the use phosphine ligands gave lower yields. The same procedure has been employed in the trifluoromethylation of analogous nickel porphyrin derivatives. ${ }^{68}$ 


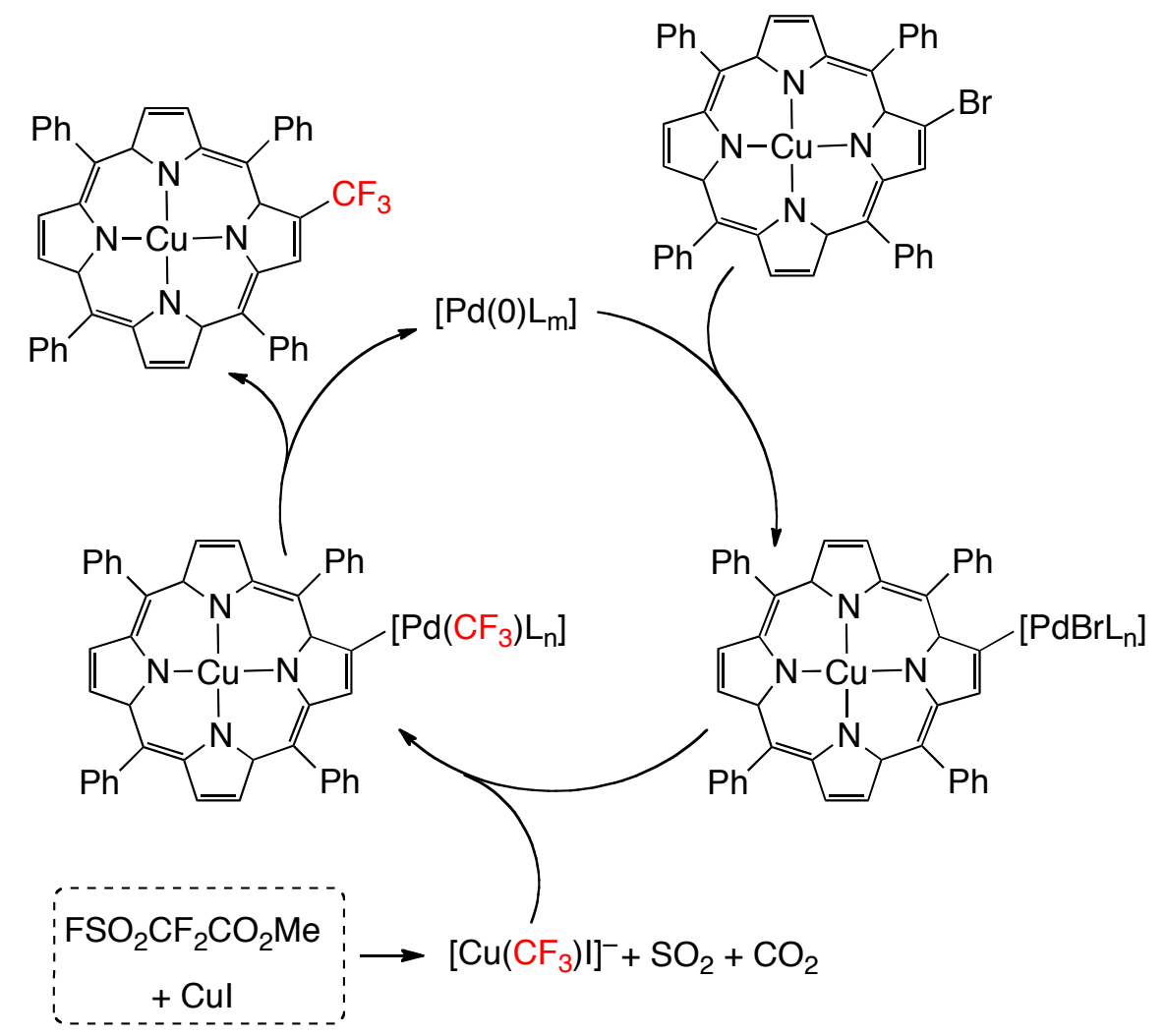

Figure 2. Proposed mechanism for the trifluoromethylation of brominated porphyrins with $\mathrm{FSO}_{2} \mathrm{CF}_{2} \mathrm{COOMe} / \mathrm{CuI}$ as trifluoromethyl source.

The mixture $\mathrm{FSO}_{2} \mathrm{CF}_{2} \mathrm{COOMe}, \mathrm{CuBr}$, and the use of $\mathrm{PdCl}_{2}$ as catalyst allow the trifluoromethylation of highly functionalized bio-active imidazol derivatives Scheme $19 .^{69}$<smiles>[R]c1nc([R7])n([R7])c1I</smiles>

Scheme 19

The copper salt is usually employed in stoichoimetric amounts or even in excess in these reactions, but there have been attempts to use this metal as co-catalyst. The trifluoromethylation of 2-aryl-1,1-dibromo-1-alkenes with an excess of $\mathrm{FSO}_{2} \mathrm{CF}_{2} \mathrm{CO}_{2} \mathrm{CH}_{3}$ (5 equivalents) in the presence of a substoichiometric amount of $\mathrm{CuI}$ 
$(30 \%)$ and $\left[\mathrm{Pd}\left(\mathrm{PPh}_{3}\right)_{4}\right](5 \%)$, led to the bis-trifluoromethylated compounds shown in Scheme 20. Under the same reaction conditions, mono-trifluoromethylated products were obtained exclusively in the case of 2-alkyl-1,1- dibromo-1-alkenes. ${ }^{70}$

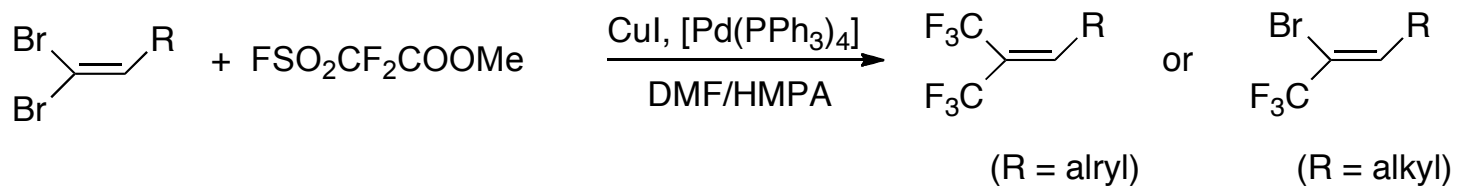

Scheme 20

In most of these bimetallic reactions the role of copper and palladium in the mechanism is not clear. Both metals are capable of activating carbon halogen bonds and to produce $\mathrm{C}-\mathrm{C}$ coupling. In fact, the copper catalyzed cross coupling reactions of aryl halides without added palladium have been profusely used and studied, many of them perfluoroakylation reactions..$^{20,62,71}$ On the other hand, the oxidative addition of aryl halides to $\operatorname{Pd}(0)$ is a well known step in many catalytic reactions, although the reductive elimination from $\mathrm{Pd}(\mathrm{II})$ complexes, also a well known process for many catalytic reactions, is specially difficult for perfluoroakyl $\mathrm{Pd}(\mathrm{II})$ derivatives. Because of this difficulty, and since it is known that $\mathrm{FSO}_{2} \mathrm{CF}_{2} \mathrm{CO}_{2} \mathrm{CH}_{3}$, is activated by copper to produce trifluoromethyl copper derivatives, Tomashenko and Grushin have proposed that the role of the palladium could be limited to the activation of the aryl halide in the oxidative activation step. This would give an aryl palladium(II) complex which can transfer the aryl group to copper to produce the elimination on this metal. ${ }^{62}$ Unfortunately, there are no studies on the transmetalation reaction of aryl or perfluoroalkyl groups between these two metals under the conditions in which the reaction can take place. 


\section{Allylic alkylation reactions}

The nucleophilic attack of fluoroalkyl stabilized carbanions generated in situ to palladium allyls has been used to synthesize fluorine-functionalized molecules. Komatsu, et al. reported the synthetic application of the palladium(0)-catalyzed allylation reactions using enolate type precursors where a methylene group is activated by fluoroalkyl and carbonyl substituents. In these systems, the 2,2,2-trifluoroethyl moiety has been introduced onto the allylic position (Scheme 21$){ }^{72}$

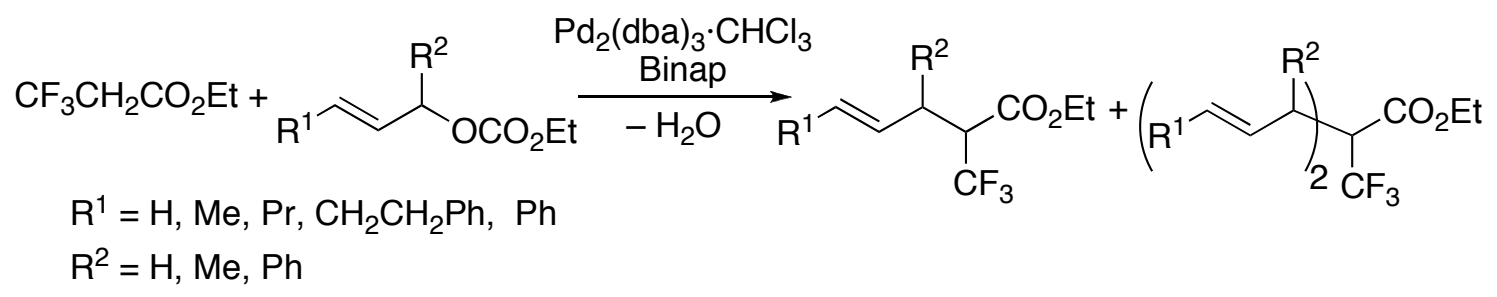

Scheme 21

1-phenylsulfonyl-2,2,2-trifluoroethane has also been used in the alkylation of allyl carbonates. $^{73}$ The reaction conditions can be controlled to produce either the mono or the diallylated alkyl. The reaction is catalyzed by $\left[\mathrm{Pd}\left(\mathrm{PPh}_{3}\right)_{4}\right]$ and the products are obtained in high yields without observation of the competing $\beta$-elimination of fluoride (Scheme 22).

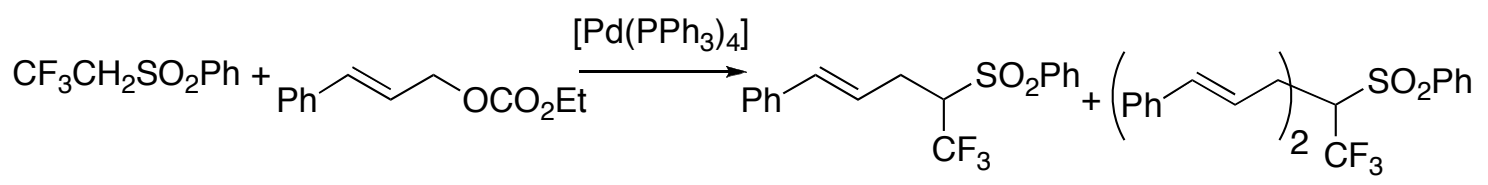

Scheme 22

Palladium catalyzed monofluoromethylation has been achieved by allyl alkylation reactions using mainly 1-fluorobis-(phenylsulfonyl)methane as nucleophile. With this reagent, the palladium catalyzed asymmetric allylic alkylation produce fluorobis(phenylsulfonyl)methylated allyl derivatives with very good enantioselectivities 
(up to $97 \%$ ee) (Scheme 23). ${ }^{74}$ The sulfonyl groups at the fluorinated carbon can be removed by reaction with $\mathrm{Mg}$, affording the chiral monofluoromethylated compounds. Monofluorinated analogs of ibuprophen, or fluorinated carbohydrates such as 5-deoxy-5fluoro- $\beta$-D-carbaribo-furanose have been synthesized applying this protocol.

$$
[\mathrm{Pd}]=\left[\mathrm{Pd}_{2}(\mu-\mathrm{Cl})_{2}\left(\mathrm{C}_{3} \mathrm{H}_{5}\right)_{2}\right] ; \mathrm{L}=(\mathrm{S}) \cdot \mathrm{PHOX}
$$

Scheme 23

$\operatorname{Pd}\left(\mathrm{PPh}_{3}\right)_{4}$ catalyzes the fluoromethylation of alkynes with monofluorinated sulfones as pronucleophiles in the presence of acetic acid (Scheme 24). ${ }^{75}$ The authors propose the alkyne transformation into an allyl-acetate that reacts with palladium and the fluoromethylating reagent in an allylic substitution pathway.

$$
\begin{aligned}
& \mathrm{R}^{1}=\mathrm{CH}_{2} \mathrm{R}^{2}+\mathrm{HFC}_{\mathrm{R}^{3}}^{-\mathrm{SO}_{2} \mathrm{Ph}} \stackrel{\left[\mathrm{Pd}\left(\mathrm{PPh}_{3}\right)_{4}\right], \mathrm{HOAc}}{1,4 \text {-dioxane }} \\
& \mathrm{R}^{1}=\operatorname{aryl} ; \mathrm{R}^{2}=\mathrm{H}, \mathrm{OMe}, \mathrm{OBn} ; \mathrm{R}^{3}=\mathrm{SO}_{2} \mathrm{Ph}, \mathrm{COPh} .
\end{aligned}
$$

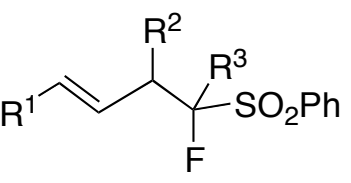

Scheme 24

\section{Catalysis through palladium mediated $C$-H activation}

In an interesting refinement of the cross-coupling reactions, Loi and Sanford reported the direct perfluoroalkylation of arenes with perfluoroalkyl iodides, using $\mathrm{Pd}_{2}(\mathrm{dba})_{3} / \mathrm{BINAP}$ as the catalytic system (Scheme 25$){ }^{76}$ This reaction proved to be resistant to several radical traps, and the yield does not change when carried out with light or in the dark, thus a radical mechanism can be ruled out. However it could not be 
clearly established whether the reaction follows a $\mathrm{Pd}(\mathrm{IV})$ pathway or if only $\operatorname{Pd}(0)$ Pd(II) species are involved.

$$
\begin{aligned}
& \mathrm{R} \frac{\sqrt{1}}{\mathrm{Cs}_{2} \mathrm{CO}_{3}}+\mathrm{R}_{\mathrm{F}} \stackrel{\mathrm{Pd}_{2}(\mathrm{dba})_{3}, \mathrm{BINAP}}{\longrightarrow} \\
& \mathrm{R}_{\mathrm{F}}=\mathrm{C}_{6} \mathrm{~F}_{13}, \mathrm{C}_{10} \mathrm{~F}_{21}
\end{aligned}
$$

Scheme 25

Feng et al. have reported the trifluoroethylation of terminal alkynes catalyzed by $\mathrm{Pd}_{2}(\mathrm{dba})_{3} / \mathrm{DPEphos}$ and using dabco (1,4-diazabicyclo[2.2.2]octane) as base, in a "copper free" Sonogashira catalysis (Scheme 26). ${ }^{77}$ The reaction tolerates a large number of different functional groups.

$$
\mathrm{R}=+\mathrm{CF}_{3} \mathrm{CH}_{2} \mathrm{I} \quad \stackrel{\mathrm{Pd}_{2}(\mathrm{dba})_{3}, \text { DPEPhos }}{\text { Dabco }} \mathrm{R} \rightleftharpoons \mathrm{CF}_{3}
$$

Scheme 26

Most of the trifluoromethylation processes involving the $\mathrm{C}-\mathrm{H}$ activation make use of electrophilic trifluoromethylating reagents, being the most commonly used the Togni's, ${ }^{78,79}$ and Unemoto's reagents (Figure 3$)^{9}$

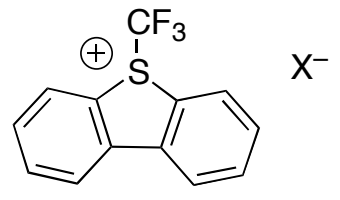

$\mathrm{X}^{-}=\mathrm{TfO}^{-}, \mathrm{BF}_{4}^{-}$.

Unemoto's reagents<smiles>CC1(C)OI(C(F)(F)F)c2ccccc21</smiles>

Togni's reagents

Figure 3. Electrophilic trifluoromethylating reagents. 
A major breakthrough was the report by the group of $\mathrm{Yu}$ of the first palladium catalyzed trifluoromethylation of arenes. ${ }^{80} \mathrm{Yu}$ et al. used the Unemoto's reagent and generated the aryl-palladium intermediate by a $\mathrm{C}-\mathrm{H}$ activation in a ortho-metalation process. The reaction requires the presence of trifluoroacetic acid and some $\mathrm{Cu}$ (II) salt that presumably acts as dibenzothiophene scavenger (Scheme 27). The reaction has been extended to the trifluoromethylation of ortho $\mathrm{C}-\mathrm{H}$ bonds in $\mathrm{N}$-arylbenzamides. ${ }^{81}$
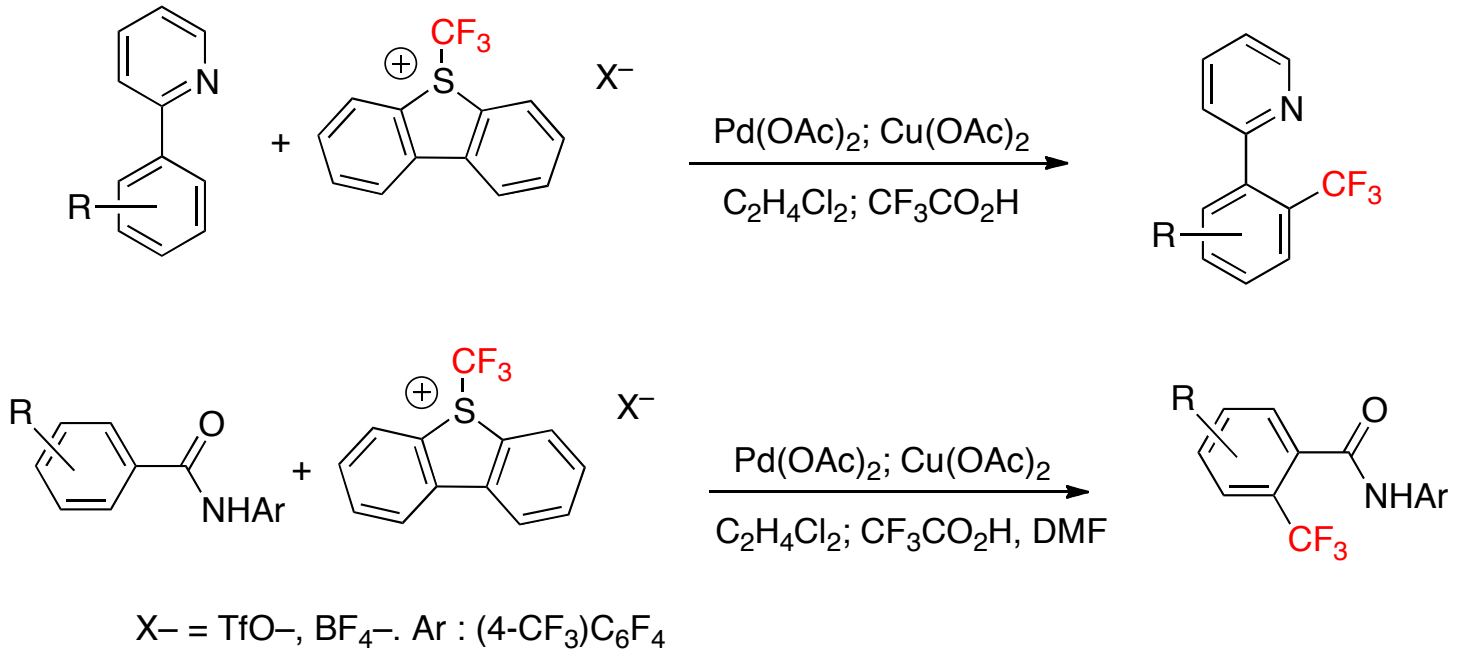

Scheme 27

The use of CsF instead of copper salts allowed the isolation of a palladium complex after the $\mathrm{C}-\mathrm{H}$ activation, whose $\mathrm{X}$-ray structure shows the coordination of the amide group that explain its role as directing groups. The trifluorometylation of this complex with the Unemoto's reagent to produce the trifluoromethyl amide requires the presence of $\mathrm{Cu}(\mathrm{AcO})_{2}$ and $\mathrm{DMF}$ to be effective. The mechanism of this reaction has not been elucidated.

$\mathrm{Mu}$ et al. reported the Pd-catalyzed oxidative trifluoromethylation of indoles using $\mathrm{Me}_{3} \mathrm{SiCF}_{3} / \mathrm{CsF}$ as trifluoromethylating reagent, $\mathrm{Pd}(\mathrm{OAc})_{2}$ as palladium source and $\mathrm{PhI}(\mathrm{OAc})_{2}$ as oxidant. ${ }^{82}$ The reaction requires the addition of an $\mathrm{N}, \mathrm{N}$ donor chelating ligand. After six hours at room temperature the reaction produces trifluorometylated 
indoles in moderate to good yield. The authors hypothesized that, as shown in Scheme 28, a reaction sequence can take place in which the $\mathrm{C}-\mathrm{H}$ bond is activated in a palladium(II) complex producing a 2-indolpalladium(II) derivative. This complex is oxidized by the strong oxidant and undergoes transmetalation from the Rupert-Prakash reagent in the presence of $\mathrm{CsF}$ to give a trifluoromethyl palladium (IV) derivative from which the reductive elimination of the trifluoromethylated indol takes place. Although the reaction sequence is quite plausible, no evidence of it was presented other than experiments that excluded a radical mechanism.

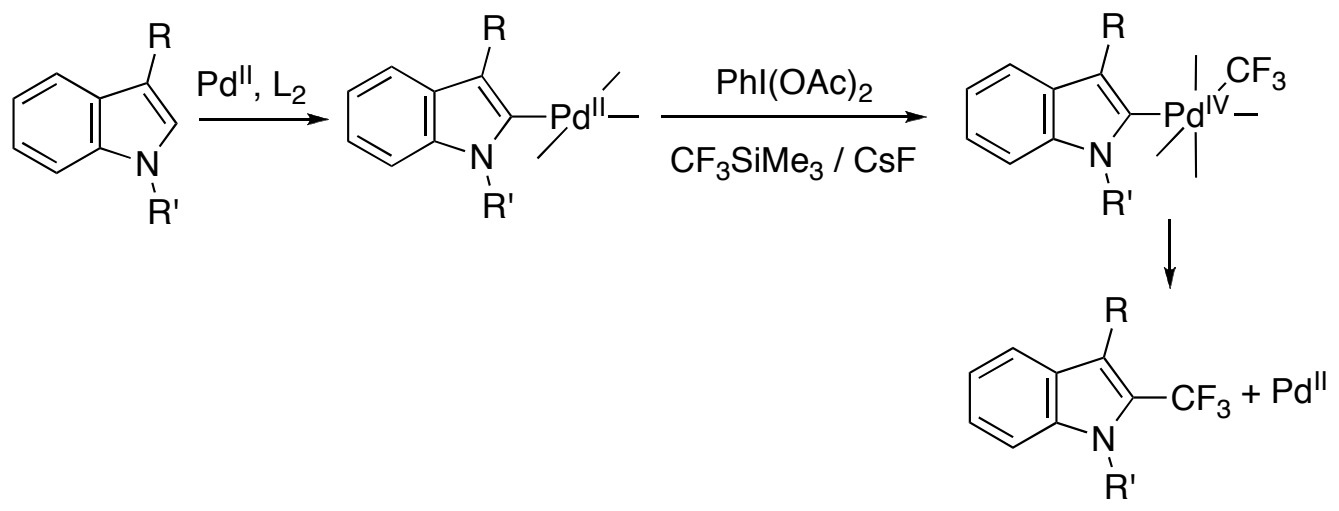

Scheme 28

In this system only hypervalent iodine reagents such as $\mathrm{PhI}(\mathrm{OAc})_{2}$ or $\mathrm{PhI}(\mathrm{OPiv})_{2}$ were effective, while oxidants incorporating the trifluoromethyl group (Togni's and Unemoto's reagents) produced lower yields. The same procedure has been applied to the trifluoromethylation of activated alkenes to produce oxindole derivatives (Scheme 29) ${ }^{83}$

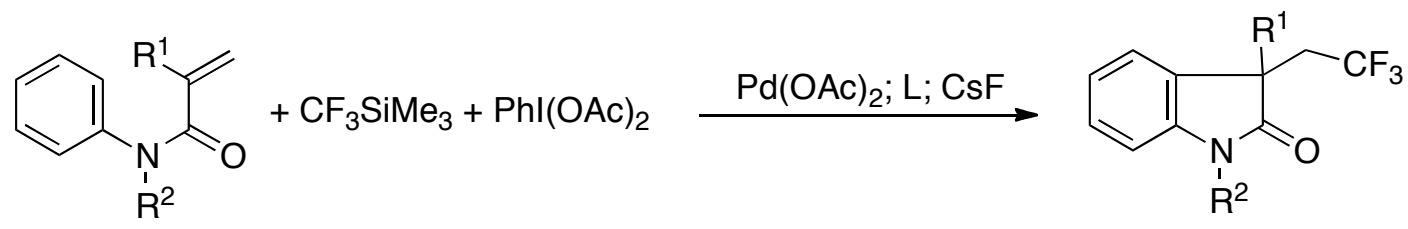

Scheme 29 
Based on spectroscopic data and ESI-MS experiments, the authors propose a reaction mechanism involving the cyclization of the amide forming a metalated $\mathrm{Pd}(\mathrm{II})$ intermediate which is oxidized by the hypervalent iodo(III) compound in the presence of $\mathrm{CF}_{3} \mathrm{SiMe}_{3} / \mathrm{CsF}$ to produce the trifluoromethyl $\mathrm{Pd}(\mathrm{IV})$ complex, which in turn yield the final product by reductive elimination step (Scheme 30).

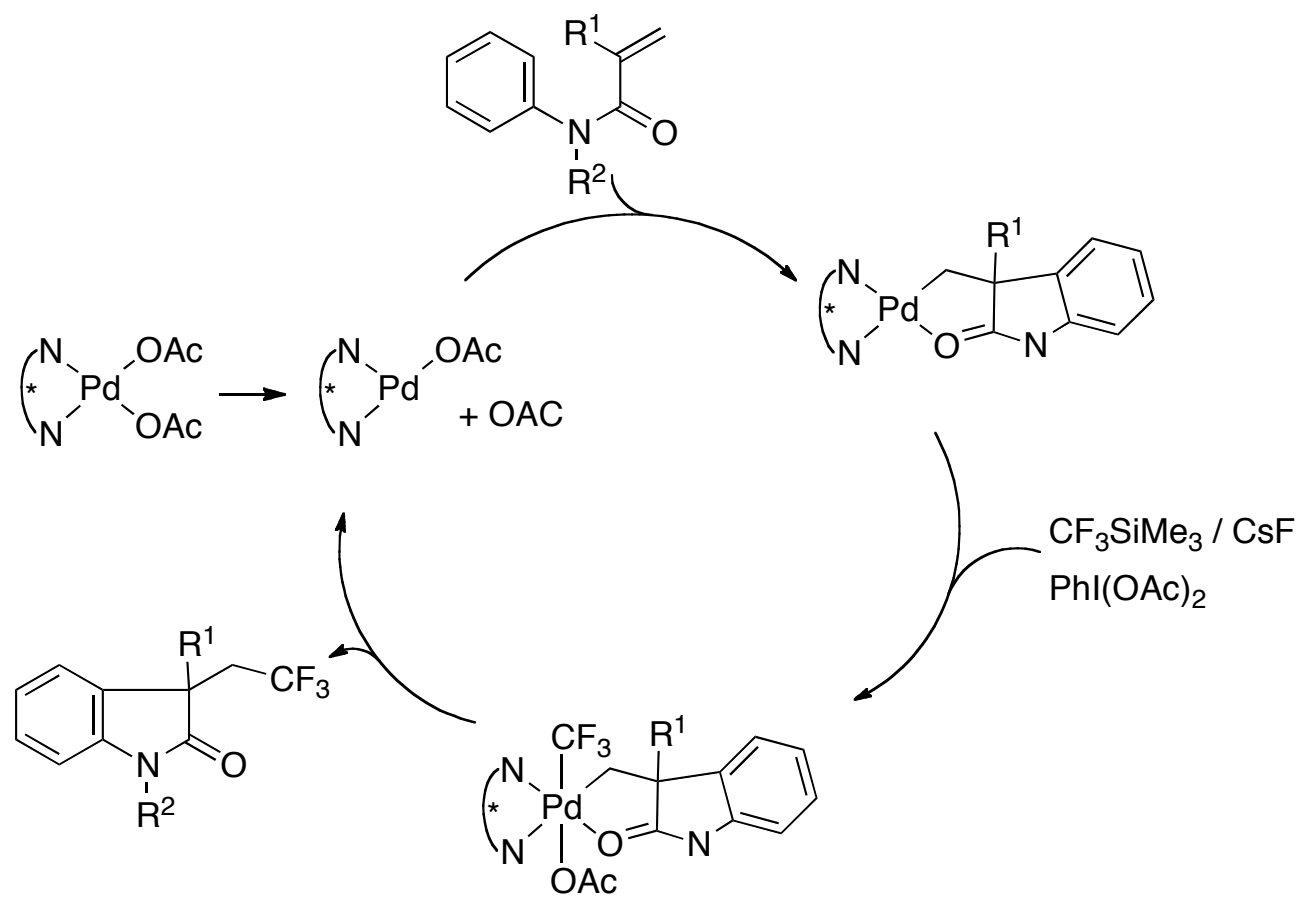

Scheme 30.

\section{Perfluoroalkylation of unsaturated molecules}

The use of the Heck reaction with fluoroalkyl complexes is quite limited due to the low reactivity of the $\mathrm{Pd}-\mathrm{Alkyl}_{\mathrm{F}}$ bond. In 1993 Filippini et al. reported the perfluoroalkylation of 1-(2-methyl prop-2-enyl)-piperidine with perfluorodecyl or perfluorododecyl iodides in ethanol. ${ }^{84}$ The catalyst used was $\left[\mathrm{PdCl}_{2}\left(\mathrm{PPh}_{3}\right)_{2}\right]$ and $\mathrm{K}_{2} \mathrm{CO}_{3}$ as base.

A more recent example is the reaction of [(Br-difluoromethyl)sulphonyl]bencene with substituted styrenes (Scheme 31). ${ }^{85}$ The reaction works with very high catalyst loadings (up to $40 \%$ of $\left[\mathrm{Pd}\left(\mathrm{PPh}_{3}\right)_{4}\right]$ ) in the presence of $\mathrm{K}_{2} \mathrm{CO}_{3}$ as 
base to yield up to $60 \%$ of the fluorinated olefin. The reaction has been extended to the synthesis of vinylfuranes, vinylthiophenes and vinylpirroles.<smiles>O=S(=O)(c1ccccc1)C(F)(F)Br</smiles><smiles>O=S(=O)(c1ccccc1)C(F)(F)Br</smiles><smiles>C=CC1=CCC=CC1</smiles><smiles>[PbH3+]</smiles><smiles>O=S(=O)(c1ccccc1)C(F)/C=C/c1ccccc1</smiles><smiles>C1=CPC=CP1</smiles><smiles>[PbH2]C1C=C[PH3+]1</smiles><smiles></smiles>

$$
E=S, O, R^{\prime}-N
$$

Scheme 31

\subsection{2. $\mathrm{C}-\mathrm{C}$ coupling of fluorinated aryl derivatives}

\section{Heck reactions}

Fluoroaryl-substituted alkenes can be prepared by the Heck reaction of fluoro and perfluorinated aryl halides with alkenes. Our group studied this reaction, long thought to be unfeasible since these reagents, specially perfluoroaryls, were considered to be too reluctant towards 1,2-insertion of alkenes into the $\mathrm{Pd}-\mathrm{Ar}_{\mathrm{F}}$ bond. ${ }^{86,87}$ Scheme 32 shows some of the reactions reported and the catalytic conditions used. A palladium complex devoid of other ligands different from the reagents, solvent and halides was used and the reaction is very efficient for styrenes and acrylates, gives moderate yields for alkyl substituted olefins and poor results for vinyl ethers. A study of the mechanism of the reaction showed that depending on the actual fluoroaryl and the reaction conditions, either the oxidative addition of the fluoroaryl bromide or the coordinationinsertion of the olefin may be rate limiting (see section 2.3.4). 


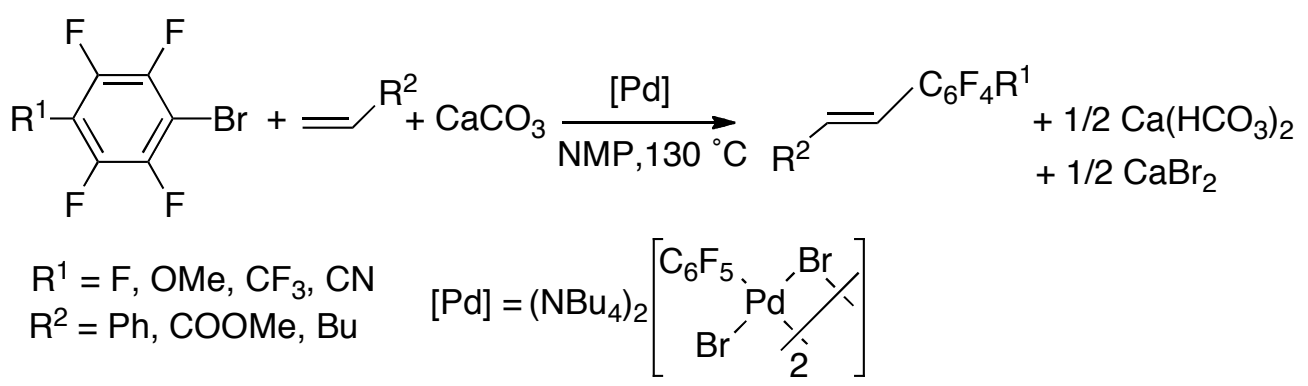

Scheme 32

An alternative approach to synthesize fluoroaryl alkenes by Heck coupling is the use of fluorinated alkenes and non-fluorinated arylhalides and this has been more frequently used. Reactions of fluorostyrenes and perfluoroalkyl ethenes with aryldiazonium salts, ${ }^{88}$ perfluroalkyl ethenes with arylhalides, ${ }^{89,90}$ and other fluoro alkene precursors have been reported. ${ }^{91,92}$

\section{Suzuki-Miyaura reactions}

The Suzuki coupling is probably one of the most extended palladium catalyzed C-C reactions involving aryl groups. Some examples of aryl-aryl coupling including a polyfluorinated $\operatorname{Ar}_{\mathrm{F}}$ groups have been reported and this has been used to synthesize complex molecules useful as materials. Thus, a combinatorial Suzuki coupling has been reported that involves polyfluorinated aryl bromides and boronic acids to synthesize molecules with liquid crystalline properties. ${ }^{93}$ The introduction of fluorine-containing groups is interesting in the design of electronic organic conducting molecules such as derivatives of oactafluoroanthracene, ${ }^{94}$ and hexabenzocoronenes, ${ }^{95}$ and this has been achieved using the Suzuki cross coupling reaction.

Selective monoarylation of mono or difluoro dibromo benzenes is possible and only one of the bromo atoms is substituted in the Pd-catalyzed reaction with boronic acids (Scheme 33). The yields of the reactions are moderate but the second possible isomer is 
not observed. The authors attribute the selectivity observed to the less steric hindrance and higher electrophilicity of the position of the substituted bromide. ${ }^{96}$<smiles>Fc1cc(F)c(Br)c(Br)c1</smiles><smiles>Fc1cc(Br)ccc1Br</smiles><smiles>Fc1ccc(Br)cc1Br</smiles><smiles>Fc1cc(F)c(Br)c(Br)c1</smiles>

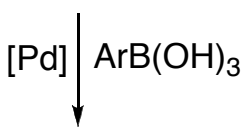<smiles>Fc1cc(Br)ccc1Br</smiles><smiles>Fc1ccc(Br)cc1Br</smiles>

Scheme 33

Many Suzuki couplings use a fluorinated aryl boronic acid as the reagent to introduce the fluoroaryl group. Partially fluorinated $\operatorname{Ar}_{\mathrm{F}} \mathrm{B}(\mathrm{OH})_{2}$ derivatives have been used, ${ }^{97}$ but as the number of fluorine atoms increase the $\operatorname{Ar}_{\mathrm{F}}$ group becomes less nucleophilic and the transmetalation step to palladium is more difficult. This situation is patent for $\mathrm{C}_{6} \mathrm{~F}_{5} \mathrm{~B}(\mathrm{OH})_{2}$ that is quite unreactive under conventional Suzuki conditions and often undergoes nucleophilic substitution of the $F_{\text {para }}$ in the presence of base. Nonetheless Korenaga, Sakai et al. found suitable reaction conditions to achieve efficient biaryl synthesis using $\mathrm{C}_{6} \mathrm{~F}_{5} \mathrm{~B}(\mathrm{OH})_{2}$ (Scheme 34). ${ }^{98}$ Key to this success is the use of $\mathrm{CsF}$ as a base, to suppress the nucleophilic fluorine substitution, and stoichiometric amounts of $\mathrm{Ag}_{2} \mathrm{O}$ which forms $\mathrm{AgX}$ ensuring the formation of electrophilic cationic palladium intermediates more prone to undergo a transmetalation process. 


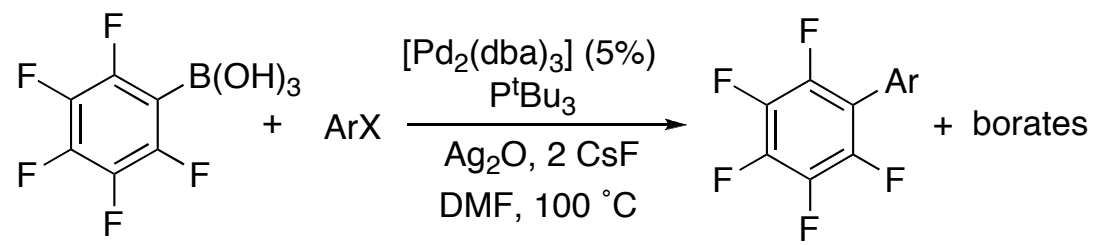

Scheme 34

Fluoroaryl trifluoroborates, $\mathrm{K}\left(\mathrm{Ar}_{\mathrm{F}} \mathrm{BF} \mathrm{F}_{3}\right)$, have also been used in the Suzuki reaction and when $\operatorname{Ar}_{\mathrm{F}}=\mathrm{C}_{6} \mathrm{~F}_{5}, \mathrm{Ag}_{2} \mathrm{O}$ is again used in the reactions either as the only base added or in combination with a carbonate salt. ${ }^{99,100,101}$

Recently, Hartwig et al. developed a very convenient formation of fluoroaryl pinacol boronates by the $\mathrm{Ir}$-catalyzed reaction of fluoroarenes and $\mathrm{B}_{2} \operatorname{pin}_{2}$ (pin $=$ pinacolate). These boronates can be isolated if needed but they can also be used in situ in a subsequent Suzuki coupling to form fluorinated biaryls (Scheme 35). ${ }^{102}$

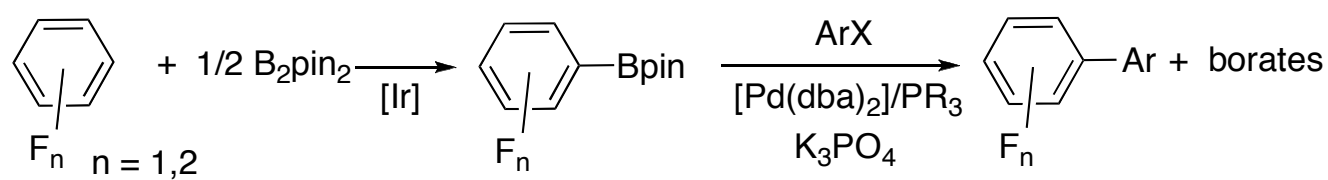

Scheme 35

\section{Stille reactions}

Few Stille reactions of polyfluoroaryl derivatives have been reported. ${ }^{103,104,105,106}$ Nonetheless it is worth noting that this coupling reaction is frequently used in the preparation of polymers and other molecules of interest in materials chemistry. In this context it is common that one of the reagents, generally the organic halide, contains one or several fluorinated positions which introduce interesting features for certain applications. ${ }^{107,108}$ The Stille coupling of pentafluorophenyliodide and a polymeric tin reagent has been reported. The fluorinated aryl is obtained in moderate yields with very low tin contamination and the stannylated polymer can be recovered and reused several 
times (Scheme 36). ${ }^{105,106}$ Stille couplings with fluoroaryl tin derivatives are also known. ${ }^{109}$

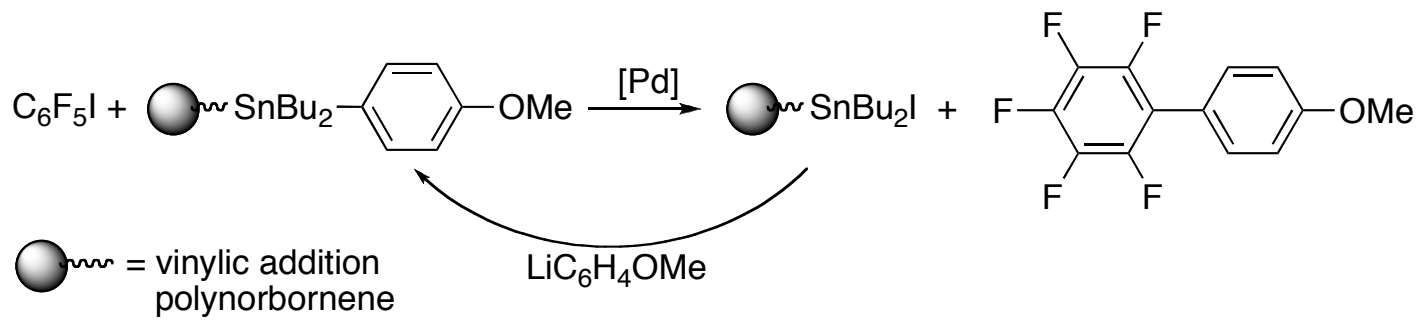

Scheme 36

\section{Sonogashira reactions}

The synthesis of fluorinated aryl alkynes via the Sonogashira reaction is not specially problematic and was reported some time ago starting from either polyfluoroaryl sulfonates, ${ }^{110}$ or polyfluoroaryl halides. ${ }^{111}$ Later reports have extended this reaction of fluorinated substrates to more complex molecules, ${ }^{94,112}$ other catalytic systems, ${ }^{113}$ or have studied the competition with other side reactions like aryl dehalogenation. ${ }^{114}$

\subsubsection{C-C coupling of fluorinated arenes.}

Reactions of fluorinated compounds where the $\mathrm{C}-\mathrm{H}$ activation occurs on the non-fluorinated reagent can be found in the literature. Coupling of fluorinated aryl halides with enolate precursors, ${ }^{115,116}$ polyfluorovinyl chlorides with thiophenes, ${ }^{117}$ and perfluorophenyl carboxylic acids with arenes have been reported. ${ }^{118}$ However, in this section, more attention will be paid to those reactions where $\mathrm{C}-\mathrm{H}$ activation occurs on the fluorinated coupling partner.

Coupling reactions of compounds that involve $\mathrm{C}-\mathrm{H}$ activation are very interesting since they do not require a previous functionalization of the reagents, making 
the process more convenient and sustainable. These reactions can be classified in different types according to the reagents that are combined and to $\mathrm{C}-\mathrm{H}$ cleavage occurring on one or both coupling partners. In most cases a $\mathrm{Pd}(0) / \mathrm{Pd}(\mathrm{II})$ catalytic cycle explains the process observed and the oxidation of $\operatorname{Pd}(0)$ to $\mathrm{Pd}(\mathrm{II})$ can be brought about by one of the reagents or by an added oxidant. According to this, two major groups of reactions can be considered: a) An organic halide or related derivative that can oxidatively add to $\operatorname{Pd}(0)$ is used as one of the reaction partners; $\mathrm{C}-\mathrm{H}$ activation occurs on the other coupling partner and no oxidizing additive is needed (A, Figure 4). b) None of the reagents is capable of oxidizing $\mathrm{Pd}(0)$ to $\mathrm{Pd}(\mathrm{II})$ because, either $\mathrm{C}-\mathrm{H}$ activation occurs in both coupling partners or the reaction combines $\mathrm{C}-\mathrm{H}$ activation on one reagent and the other one is only capable of reacting with $\mathrm{Pd}(\mathrm{II})$ (as main group organometallics for example). These reactions require the presence of an oxidant as additional reagent (B, Figure 4). Coupling reactions belonging to either group have been described for fluorinated derivatives. If strong oxidants are used the mechanism can shift to a $\mathrm{Pd}(\mathrm{II}) / \mathrm{Pd}(\mathrm{IV})$ cycle as occurs for $\mathrm{C}-\mathrm{H}$ activation in the presence of iodine(III) derivatives or electrophilic fluoroalkylating reagents as shown above (section 2.1.1).
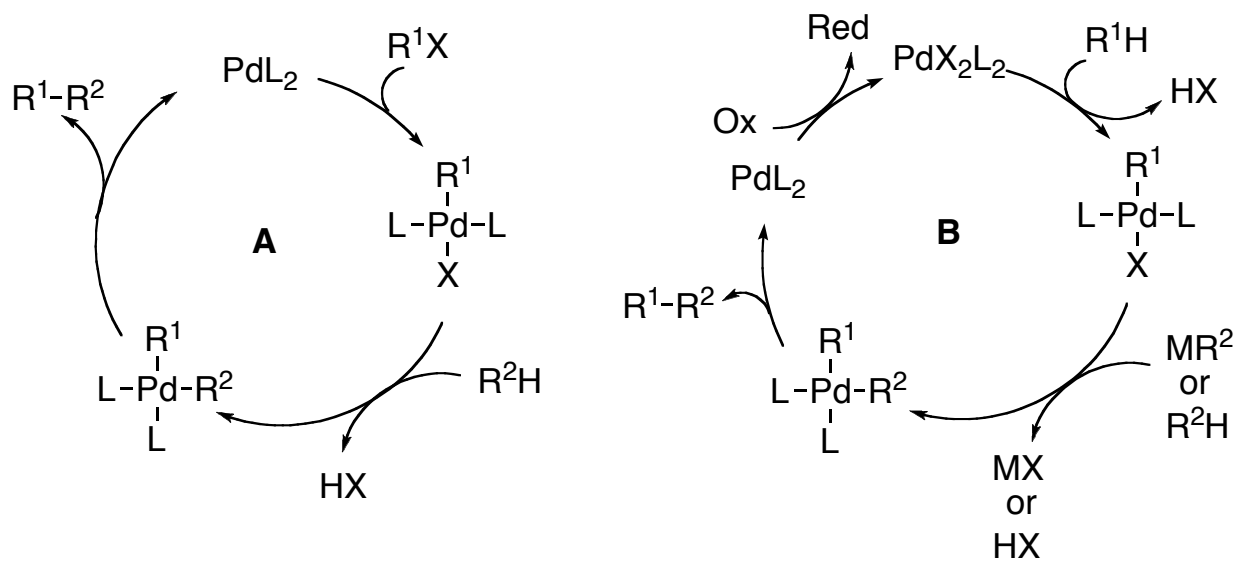

Figure 4. Schematic representation of the common operating catalytic cycles in C-H activation reactions of arenes: non-oxidative (A) and oxidative (B). 
Fagnou et al. described the first examples of coupling reactions between an aryl halide and pentafluorobenzene or other substituted tetrafluorobenzenes (Scheme 37). ${ }^{119}$ The reaction avoids the need to use polyfluorinated organometallic derivatives and works with a wide variety of aryl iodides, including those sterically hindered. ${ }^{120}$ An electrophilic aromatic susbstitution mechanism $\left(\mathrm{S}_{\mathrm{E}} \mathrm{Ar}\right)$ had been traditionally proposed for the metalation of electron rich arenes by palladium, mostly in orthometalation reactions. However, the electronic features of pentafluorobenzene do not favor such a mechanism. Experimental mechanistic data and calculations on the system led the authors to propose a concerted metalation-proton abstraction to a base as the plausible $\mathrm{C}-\mathrm{H}$ activation pathway (Scheme 37). This mechanism is also favored for other arenes. $^{121,122}$

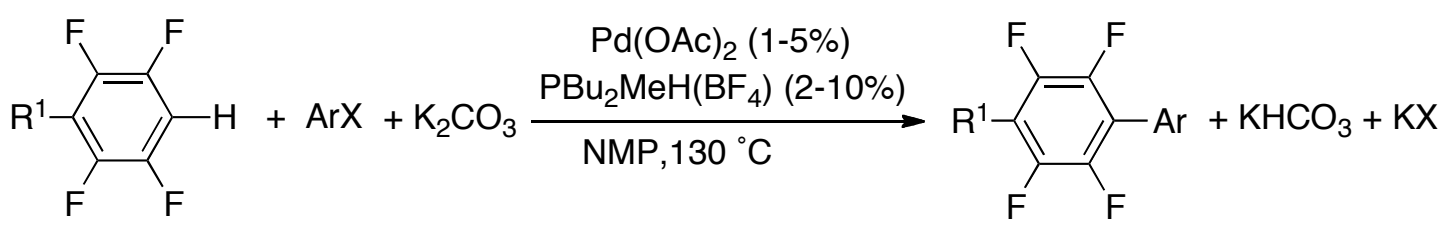

$\mathrm{R}^{1}=\mathrm{F}, \mathrm{OMe}, \mathrm{Me}, \mathrm{H}$ $\mathrm{X}=\mathrm{Br}, \mathrm{I}$

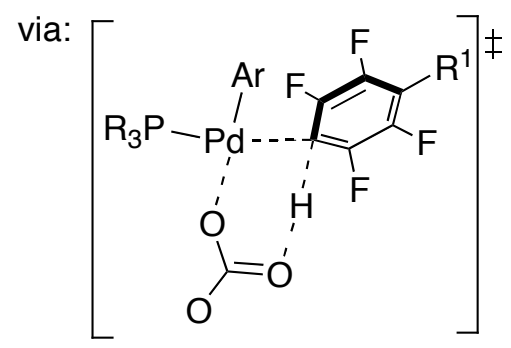

Scheme 37

Later on, Zhang et al. have extended this reaction using other organic derivatives instead of ArX. Thus new polyfluoroaryl substituted compounds have been prepared by reaction of polyfluoroarenes and aryl tosylates, ${ }^{123}$ allylic halides, ${ }^{124,125}$ allylic esters, ${ }^{126}$ and heteroaryl halides. ${ }^{127}$

In 2007, Fagnou et al. reported the direct coupling of an arene and indol derivatives by double C-H activation. ${ }^{128}$ Since then, the reaction has been extended to 
many organic compounds, including fluorinated arenes (Scheme 38). The catalytic cycle involves two $\mathrm{C}-\mathrm{H}$ activations on $\mathrm{Pd}(\mathrm{II})$ centers to give a $\left[\mathrm{PdAr}_{\mathrm{F}} \mathrm{R}^{2} \mathrm{~L}_{2}\right]$ intermediate that gives the final product by reductive elimination. The $\operatorname{Pd}(0)$ complex formed needs to be reoxidize to $\mathrm{Pd}(\mathrm{II})$ and for this reason, the reaction employs a stoichiometric amount of an oxidant. Copper(II) salts have been used for this purpose as well as silver derivatives, often $\mathrm{Ag}_{2} \mathrm{CO}_{3}$ or $\mathrm{Ag}_{2} \mathrm{O}$ which also introduce a base needed for most transformations. As shown in Scheme, this oxidative coupling has been reported for polyfluoroarenes reacting with arenes, ${ }^{129,130}$ heterocycles. ${ }^{131}$ Recently, the coupling of polyfluoroarenes and thiophenes has been carried out using catalytic amounts of $\operatorname{Ag}_{2} \mathrm{O}$ and oxygen as the oxidant which is a substantial improvement in this oxidative coupling. ${ }^{132}$

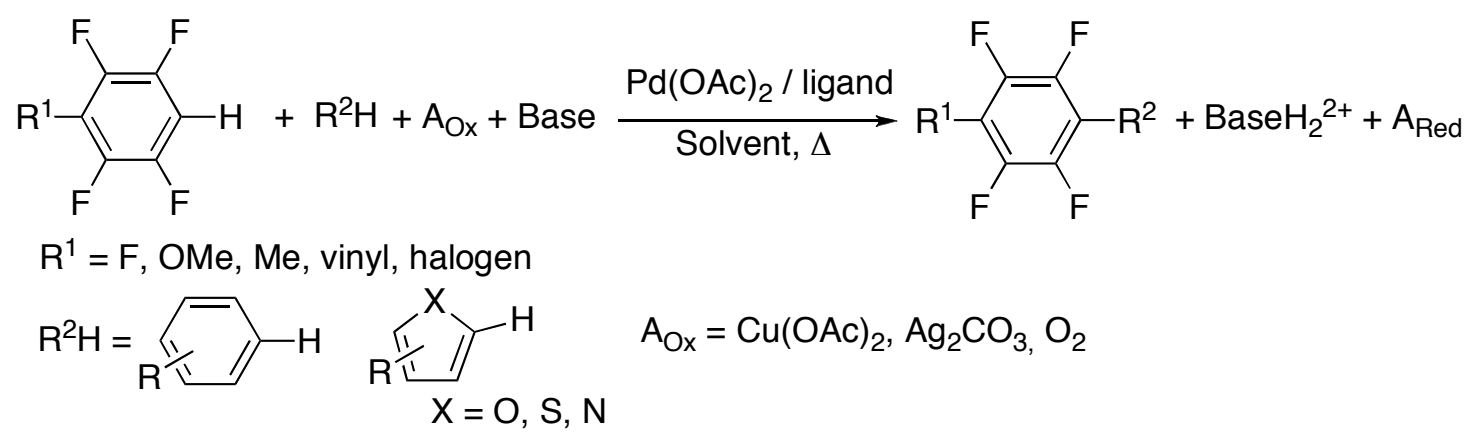

Scheme 38

Oxidative Heck couplings, i.e. a Heck reaction between an arene and an alkene, have been reported for polyfluoroarenes and the reaction can be applied to alkenes with electron withdrawing substituents or 2-alkyl olefins (Scheme 39). ${ }^{133}$ Allylic esters also undergo this reaction under the oxidative conditions shown in Scheme 39. ${ }^{134}$ It is noteworthy that in the absence of an oxidant, oxidative addition of the allylic ester to $\operatorname{Pd}(0)$ can occur and the reaction outcome is different with loss of the carboxylate fragment and the allylic substitution of the arene (as in Scheme 37 but allylX instead of ArX). ${ }^{126}$ 


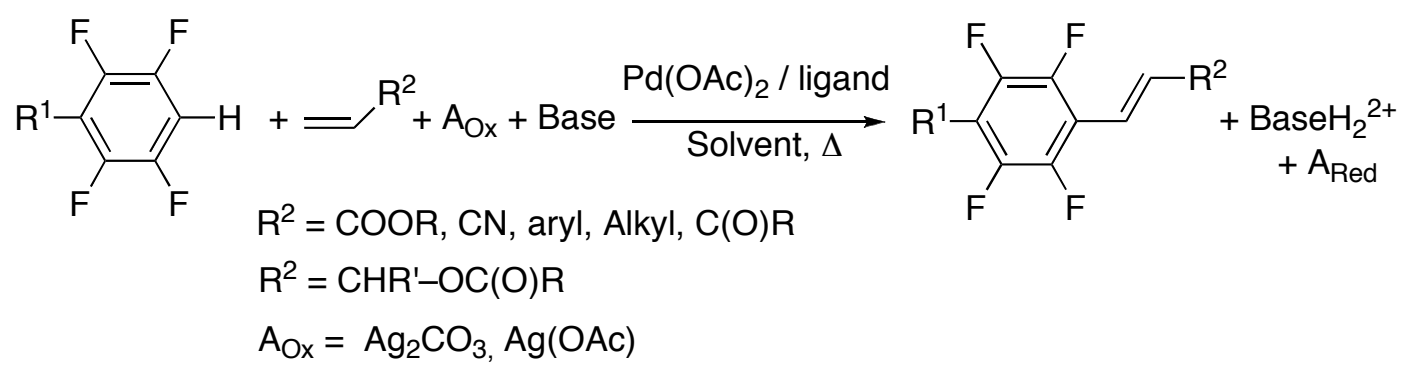

Scheme 39

The oxidative coupling of polyfluoroarenes with a arylfluoroborates has been reported. ${ }^{135}$ The reaction of aromatic carboxylic acids $(\mathrm{ArCOOH})$ and polyfluoroarenes $\left(\mathrm{Ar}_{\mathrm{F}} \mathrm{H}\right)$ lead to the synthesis of biaryls. ${ }^{136}$ The reaction occurs under oxidative conditions since both arylations, to give the intermediate [PdAr $\left.\operatorname{PrL}_{2}\right]$, occur on $\mathrm{Pd}(\mathrm{II})$ derivatives: coordination of $\mathrm{ArCOO}^{-}$and decarboxylation for the Ar fragment and subsequent $\mathrm{C}-\mathrm{H}$ activation for $\mathrm{Ar}_{\mathrm{F}}$.

\subsubsection{C-C coupling of fluorinated alkenyls.}

Most Pd-catalyzed reactions reported for fluoroalkenyl derivatives are Negishi and Stille couplings. Examples of the other common Pd-catalyzed reactions can be found but to a less extent. Only reactions of perfluorinated alkenyls or $\alpha$ fluorosubstituted derivatives will be mentioned here; the reactions of compounds with fluoro-substituents far from the carbon where the new bond is formed have been left aside.

Barton et al. reported a general method for the synthesis of fluorostyrenes by the Negishi coupling of aryl halides and poly- or perfluorovinyl zinc halides (Scheme 40). ${ }^{137,138}$ This type of reagents have been used to introduce fluorovinyl fragments in other substrates such as uracil, ${ }^{139}$ porphyrins, ${ }^{140}$ and ferrocene ${ }^{141}$ 


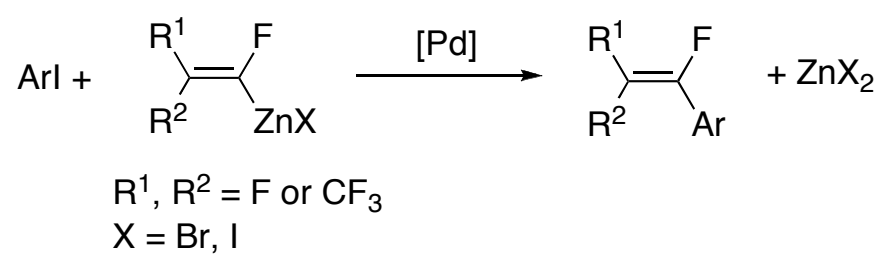

Scheme 40

The coupling of fluorinated vinylic and allylic halides with perfluorovinylzinc reagents has been carried out, although the yields obtained are lower than those reported for the analogous Stille coupling. ${ }^{142}$ The synthesis of fluorinated alkenes by reaction of a fluorovinyl halide and a non-fluorinated zinc organometallic compound has also been reported. ${ }^{143,144}$

Fluorovinyl tin compounds are known and have been used in the Stille reaction with non-fluorinated aryl halides, acylhalides and other organic halides (Scheme 41). ${ }^{141,145-148}$ Some other reactions combine a fluorovinyl stannane with fluorinated alkenyl halides in the presence of a palladium catalyst. ${ }^{142,149}$ Fluorinated dienes have been synthesized in this way and, in some cases, the addition of a copper salt as cocatalyst has been used. ${ }^{148,149}$

$$
\begin{aligned}
& \mathrm{R}^{1} \mathrm{X}+\underset{\mathrm{R}^{3}}{\mathrm{\textrm {R } ^ { 2 }}}=\left\langle\underset{\mathrm{SnR}_{3}}{\mathrm{~F}} \stackrel{[\mathrm{Pd}]}{\longrightarrow} \underset{\mathrm{R}^{3}}{\mathrm{R}^{2}}=\left\langle_{\mathrm{R}^{1}}^{\mathrm{F}}+\mathrm{SnR}_{3} \mathrm{X}\right.\right. \\
& \mathrm{R}^{1}=\text { aryl, acyl, } \mathrm{CF}_{2}=\mathrm{CF} \quad \mathrm{R}^{2}, \mathrm{R}^{3}=\mathrm{F}, \mathrm{CF}_{3} \text {, etc. } \\
& \mathrm{X}=\mathrm{Br}, \mathrm{I}
\end{aligned}
$$

Scheme 41

Most reactions employ a preformed stannane but in some cases the organometalic tin derivative is formed in situ, as in the synthesis of symmetrical fluorinated dienes from fluoroalkenylhalides and hexaalkylditin (Scheme 42). ${ }^{150}$ 


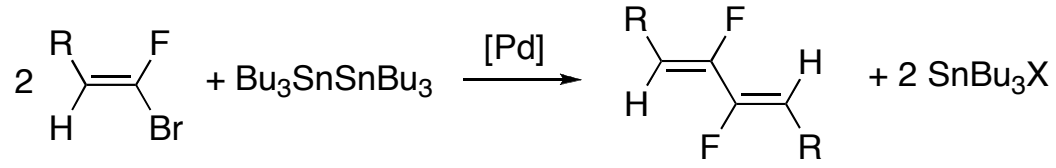

Scheme 42

Distannanes have been used in the synthesis of fluorinated polymers, specially polyarylenevinylenes which show interesting optical properties (Scheme 43). ${ }^{151,152}$ For example, the presence of fluorine atoms in the structure produces a shift in the emission wavelength of these polymers to the visible region.

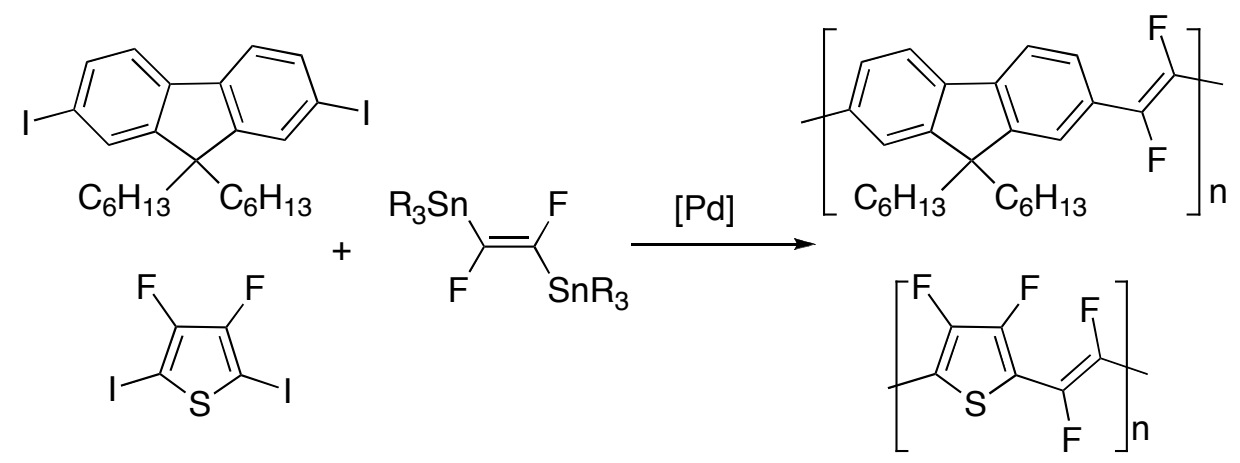

Scheme 43

Other Pd-catalyzed coupling reactions that use fluorinated alkenyl halides as substrates can be found in the literature and generally the coupling partner is a nonfluorinated molecule. Thus, examples of Suzuki reactions for the synthesis of fluoroalkenyl substituted arenes involve a fluorinated alkenyl bromide or iodide and a non-fluorinated arylboronic acid. ${ }^{153-155}$ In a series of related papers Barton et al. and others reported some time ago the synthesis of fluorinated enynes by reaction of fluorovinyl iodides or bromides with alkynes in the presence of a palladium complex and a copper(I) salt, i.e. a Sonogashira coupling. ${ }^{156-158}$ Fluorinated vinyl halides have been transformed into fluorinated formyl derivatives by a Pd-catalyzed carbonylation 
reaction. ${ }^{159} \mathrm{~A}$ Pd-catalyzed benzannulation reaction of fluorinated enynes with dialkynes leads to fluoroaromatic derivatives (Scheme 44). ${ }^{160}$

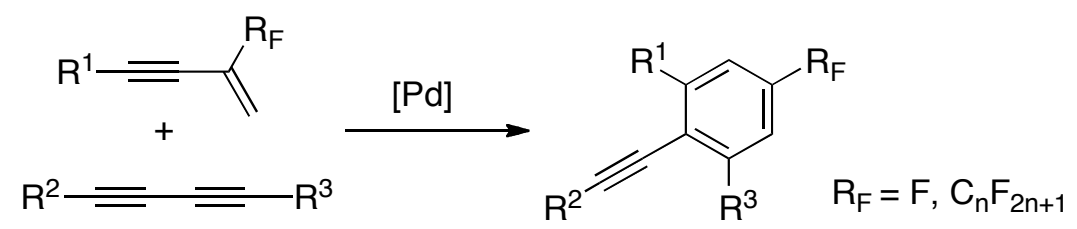

Scheme 44

Allylic substitution reactions on fluorinated substrates have been reported where either the allylic fragment is the fluorinated one and the product is a fluorinated alkene (Scheme 45, a), ${ }^{161}$ or the nucleophile contains a fluorine atom as in the enantioselective allylation of enol ethers described by Paquin et al. that produces fluoroallyl derivatives (Scheme 45, b). ${ }^{162}$

a)

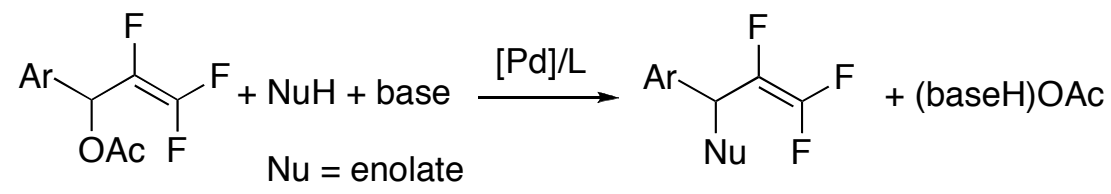

b)

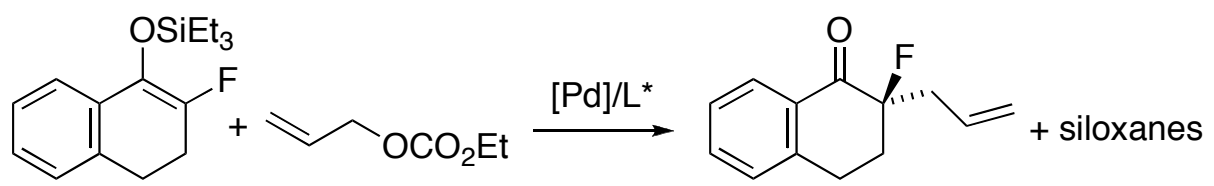

Scheme 45

\subsection{The Pd-R Fond}

\subsubsection{Pd-Alkyl bonds}

The perfluoroalkyl-metal bond is quite different from other $\mathrm{M}-\mathrm{C}$ bonds, being the most relevant distinct feature its chemical inertness, that is particularly striking in the case of trifluoromethylpalladium(II) complexes. In spite of the expected large 
electronegativity of the trifluoromethyl group, that should decrease its donor capability when compared with $\mathrm{CH}_{3}$, the trans effect of the $\mathrm{CF}_{3}$ group is similar to that of the methyl group, and the C-M bond distances are also the same within the experimental error. In addition, the group does not participate easily in reductive elimination processes neither in $\mathrm{Pd}(\mathrm{II})$ nor in $\mathrm{Pd}(\mathrm{IV})$ complexes. These properties are quite general, valid for other transition metal trifluoromethyl derivatives and have caught the eye of several theoretical and computational chemists.

Algarra et al. have performed a DFT study of a series of trifluoromethyl complexes including $\left[\mathrm{Pd}\left(\mathrm{CF}_{3}\right) \mathrm{Cl}(\mathrm{dppe})\right],{ }^{163,164}$ but also complexes of other metals, such as platinum(II), rhodium(I), nickel(II), copper(I), gold(I), and manganese(I), and compare the calculated structural features of these complexes with the analogous methyl derivatives. Table 1 gathers the experimental and calculated data for the palladium complexes. It is shown in this study that the $\mathrm{Pd}-\mathrm{C}$ bond distances to $\mathrm{CF}_{3}$ and to $\mathrm{CH}_{3}$ are almost the same, and the distances Pd-P corresponding to the phosphorous atom trans to $\mathrm{CX}_{3}$ are almost equal for both groups. Thus, a comparable trans influence is exerted by both groups. Also, it is quite relevant the analysis of the charge distribution in these complexes. In spite of the electron withdrawing properties of the $\mathrm{CF}_{3}$ group, the metal bonded to it has less positive charge than the metal bonded to the $\mathrm{CH}_{3}$, although the opposite rules for the carbon atoms. The charge at the phosphorus trans to the methyl group is the same in both complexes. This behavior is not particular for the palladium and in other metals a analogous charge distribution was found. 
Table 1. Experimental and calculated parameters for $[\mathrm{PdRCl}(\mathrm{dppe})]$ complexes $(\mathrm{R}=$ $\left.\mathrm{CF}_{3}, \mathrm{CH}_{3}\right)$.

\begin{tabular}{|l|l|l|l|l|l|}
\hline Complex & $\begin{array}{l}\mathrm{d}(\mathrm{Pd}-\mathrm{C})(\AA) \\
(\text { calcd/exp })\end{array}$ & $\begin{array}{l}\mathrm{d}\left(\mathrm{Pd}-\mathrm{P}_{\text {trans }}\right)(\AA) \\
(\text { calcd/exp })\end{array}$ & $\mathrm{q}_{\mathrm{M}}{ }^{\mathrm{a}}$ & $\mathrm{q}_{\mathrm{C}}{ }^{\mathrm{b}}$ & $\mathrm{q}_{\text {Ptrans }}{ }^{\mathrm{c}}$ \\
\hline$\left[\mathrm{Pd}\left(\mathrm{CH}_{3}\right) \mathrm{Cl}(\right.$ dppe $\left.)\right]$ & $2.089 / 2.135(4)$ & $2.379 / 2.376(1)$ & +0.23 & -0.89 & +0.45 \\
\hline$\left[\mathrm{Pd}\left(\mathrm{CF}_{3}\right) \mathrm{Cl}(\right.$ dppe $\left.)\right]$ & $2.048 / 2.084(3)$ & $2.374 / 2.345(1)$ & +0.19 & +0.82 & +0.47 \\
\hline
\end{tabular}

(a) Calculated charge at the metal. (b) Calculated charge at the carbon. (c) Calculated charge at the phosphorus trans to the $\mathrm{CF}_{3}$ or the $\mathrm{CH}_{3}$ group.

In addition the authors performed a complete NBO (Natural Bond Analysis) of the $\mathrm{M}-\mathrm{CF}_{3}$ in the model complex $\left[\mathrm{Pt}\left(\mathrm{CF}_{3}\right) \mathrm{H}_{3}\right]^{2-}$, and compared it with the same analysis for the analogous methyl model complex $\left[\mathrm{Pt}\left(\mathrm{CH}_{3}\right) \mathrm{H}_{3}\right]^{2-}$. The conclusions of this study agree with the calculation and experimental data obtained by Yang et al. in $1990 .{ }^{165}$ They found that one effect of the $\mathrm{CF}_{3}$ group is to stabilize all the Pt-based lone pairs by ca. $0.02 \mathrm{au}$, what is consistent with a general field stabilization of the metal-based orbitals (Figure 5). A major change in going from $\mathrm{CH}_{3}$ to $\mathrm{CF}_{3}$ is the hybridization of the carbon: The orbital describing the electron pair bonded to the metal changes from being $\mathrm{sp}^{3.15}$ in the former to $\mathrm{sp}^{1.88}$ in the $\mathrm{CF}_{3}$ that produces a stabilization on the molecular orbital involved in the C-M $\sigma$ bond. An additional stabilization came from the relatively low energy of the antibonding C-F orbitals when compared with the $\sigma^{*} \mathrm{C}-\mathrm{H}$, which facilitates their participation in the $\sigma-\mathrm{CF}_{3}-\mathrm{M}$ bonding. The energy of these orbitals in comparison with those of the methyl analogue is shown in Figure 5. The retrodonation effects are less important: For the platinum complex the $\pi$-bacbonding $\mathrm{M} \rightarrow \mathrm{CF}_{3}$ has been estimated to be only $8 \%$ of the strength of the $\sigma \mathrm{CF}_{3}-\mathrm{M}$.

These works explain the stabilizing effect of the fluorine substituents and for this reason the chemical inertness of the perfluoroalkyl groups bound to a metal. 


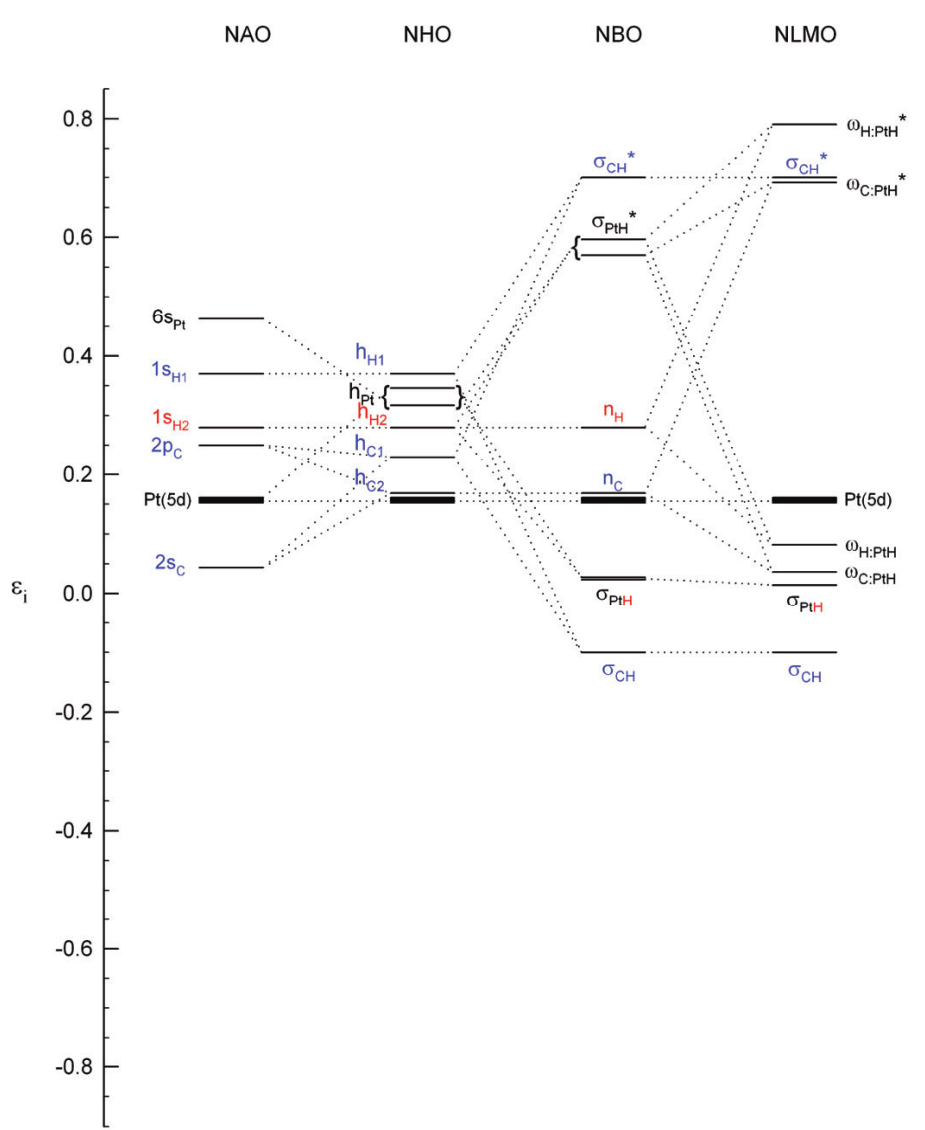

$\left[\mathrm{PtH}_{3}\left(\mathrm{CH}_{3}\right)\right]^{2-}$

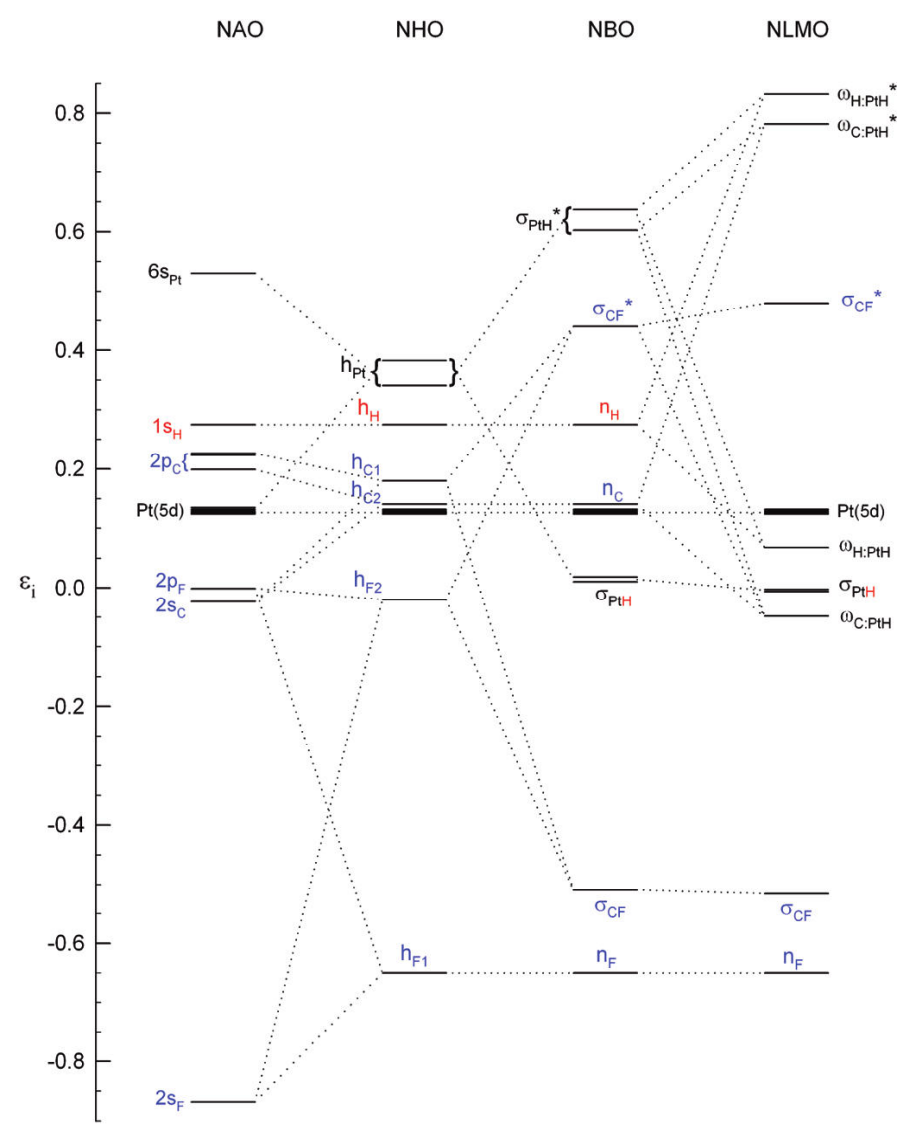

$\left[\mathrm{PtH}_{3}\left(\mathrm{CF}_{3}\right)\right]^{2-}$

Figure 5. Computed energy correlation diagrams (au) for the NAO $\rightarrow \mathrm{NHO} \rightarrow \mathrm{NBO} \rightarrow \mathrm{NLMO}$ (natural atomic orbitals $\rightarrow$ natural hybrid orbitals $\rightarrow$ natural bond orbitals $\rightarrow$ natural localized molecular orbitals) transformations in $\left[\mathrm{PtH}_{3}\left(\mathrm{CH}_{3}\right)\right]^{2-}$ (left) and $\left[\mathrm{PtH}_{3}\left(\mathrm{CH}_{3}\right)\right]^{2-}(\mathrm{right})$. In both diagrams the Pt(5d) NAOs and the subsequent Pt-based orbitals not involved in bonding are shown by a single heavy line at their average energy; an average energy is also shown for any set of three near-degenerate orbitals involved in $\mathrm{CX}_{3}$ interactions. Reprinted with permission from reference 164 . Copyright 2011 American Chemical Society 


\subsubsection{Pd-Ar F bonds}

Metal-fluoroaryl bonds are stronger than the analogous M-aryl bonds and the higher ionic character of the $\mathrm{M}-\mathrm{Ar}_{\mathrm{F}}$ bond has been deemed responsible. Indeed, the electron withdrawing ability of $\mathrm{C}_{6} \mathrm{~F}_{5}$ is higher than that of a phenyl group. ${ }^{166}$ More recently, Perutz, Eisenstein et al. have analyzed the strength $\mathrm{M}-\mathrm{Ar}_{\mathrm{F}}$ bond by DFT calculations for different metals, ${ }^{167}$ and also for palladium. ${ }^{168}$ The effect of fluorine substitution in the ring affects the charge distribution and increases the polarity of the M-C bond, i.e its ionic character, making the bond stronger, in accordance with the accepted view. The number and position of the fluorine atoms in the ring affect the $\mathrm{M}-\mathrm{C}$ dissociation energy and the calculated $\mathrm{M}-\mathrm{Ar}_{\mathrm{F}}$ bond dissociation energy relative to $\mathrm{M}$ $\mathrm{C}_{6} \mathrm{H}_{5}\left(\Delta \mathrm{D}(\mathrm{M}-\mathrm{C})_{\text {rel }}\right)$ follow the linear equation: $\left.\Delta \mathrm{D}(\mathrm{M}-\mathrm{C})_{\text {rel }}\right)=a+b \mathrm{n}_{\text {ortho }}+c\left(\mathrm{n}_{\text {meta }}+\mathrm{n}_{\text {para }}\right)$ $\mathrm{kJ} \mathrm{mol}^{-1}$, where $\mathrm{n}$ is the number of $\mathrm{F}$ atoms at the given positions and $a-c$ are the increments in relative metal aryl bond energy. The coefficients $a-c$ have different values, and $b$ is about $20-30 \mathrm{~kJ} \mathrm{~mol}^{-1}$ whereas $c$ is eight to ten times lower and $a$ is negligible. This means that the $F_{\text {ortho }}$ have the largest influence in making the $M-A_{F}$ stronger. It has been found that there is a linear correlation between the $\mathrm{C}-\mathrm{H}$ and $\mathrm{M}-\mathrm{C}$ bond energy for the arenes and the analogous organometallic complexes. This correlation has a slope close to 2 , indicating that although the $\mathrm{C}-\mathrm{H}$ bond of fluorinated arenes is stronger that of $\mathrm{C}_{6} \mathrm{H}_{6}$, the energy difference between them $\left(\Delta \mathrm{D}(\mathrm{C}-\mathrm{H})_{\text {rel }}\right)$ is lower than $\left(\Delta \mathrm{D}(\mathrm{M}-\mathrm{C})_{\text {rel }}\right)$ so there is a thermodynamic advantage in the formation of a $\mathrm{M}-\mathrm{Ar}_{\mathrm{F}}$ bond from $\mathrm{Ar}_{\mathrm{F}} \mathrm{H}$. In this way, the reactions that involve the formation of $\mathrm{a} M-\mathrm{Ar}_{\mathrm{F}}$ bond will be favored whereas those where the $\mathrm{M}-\mathrm{Ar}_{\mathrm{F}}$ needs to be cleaved are expected to be slower. When different metal complexes were analyzed, these correlations are not affected by the electron count on the metal, so $\pi$-effects should be considered unimportant. 
A complete calculation on the palladium catalyzed direct arylation of fluoroarenes shown in Scheme 37 illustrates those findings. ${ }^{168}$ Two important transition states for the reaction were calculated for different isomeric di- tri- tetra- and pentafluoroarenes, corresponding to the $\mathrm{C}-\mathrm{H}$ cleavage (A, Scheme 46) and the reductive elimination steps (B, Scheme 46). The lowest energy for the first one corresponds to $\mathrm{C}_{6} \mathrm{~F}_{5} \mathrm{H}$, the presence of $\mathrm{F}_{\text {ortho }}$ having the stronger influence and determining the selectivity of the reaction: when several isomers can be formed, there is a strong preference for activating the $\mathrm{C}-\mathrm{H}$ bond ortho to the fluorine atoms, so the stronger $\mathrm{M}$ $\operatorname{Ar}_{\mathrm{F}}$ bond is formed. In the second one (B, Scheme 46), the opposite is observed and the higher barrier is found for those fluoroaryl with ortho-fluorine substitution.

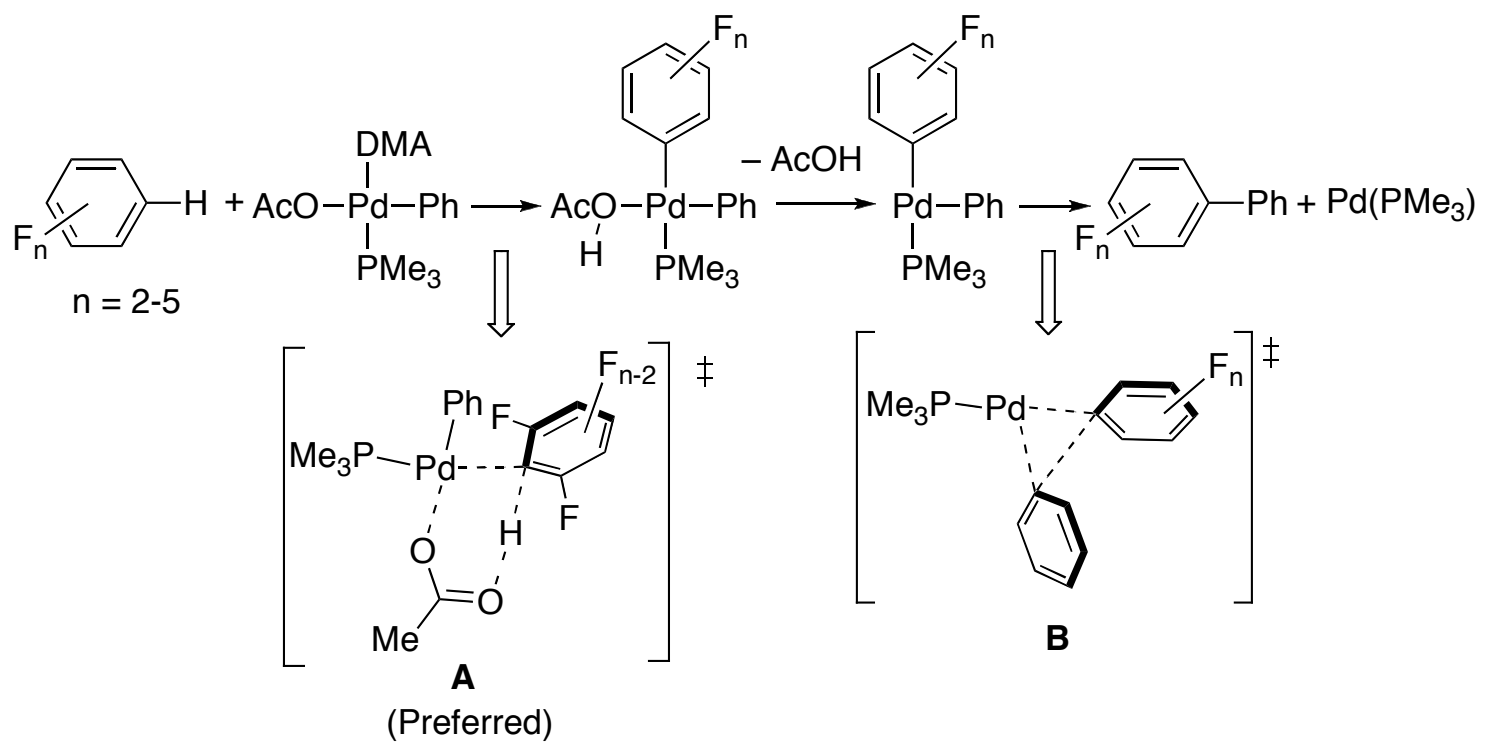

Scheme 46

As a result of the strong $\mathrm{M}-\mathrm{Ar}_{\mathrm{F}}$ bonds, fluoroaryls allow to isolate stable organometallic complexes, and a large number of fluoroaryl palladium derivatives are known. ${ }^{21,27}$ As just mentioned, they show slower kinetics than aryl complexes in reactions involving the cleavage of the $\mathrm{M}-\mathrm{Ar}_{\mathrm{F}}$ bond. These features are ideal for the study of reaction mechanisms by isolating intermediates and analyzing elementary steps 
separately, which are the basis for the understanding of catalytic processes and some examples will be given below.

\subsection{Elementary steps in organofluorine $\mathrm{C}-\mathrm{C}$ coupling palladium}

\section{catalyzed processes.}

Because of the unique properties of fluorine in NMR spectroscopy, fluoroaryl palladium complexes have been used to monitor reactions, many of them under catalytic conditions, and to identify intermediates in complex reactions systems. In addition, the stability of fluoroaryl palladium derivatives and their slow kinetics in reactions involving the cleavage of the $\mathrm{M}-\mathrm{Ar}_{\mathrm{F}}$ bond allow the isolation of reaction intermediates that can be detected rarely when non-fluorinated aryls are used. These features have been applied to the study of fundamental organometallic reactions that are important steps in catalytic transformations as shown below. A simplified catalytic cycle for a C-C coupling reaction shows some of this fundamental steps in Figure 5; other C-C forming reactions such as insertion of unsaturated substrates into $\mathrm{Pd}-\mathrm{R}_{\mathrm{F}}$ bonds are also included.

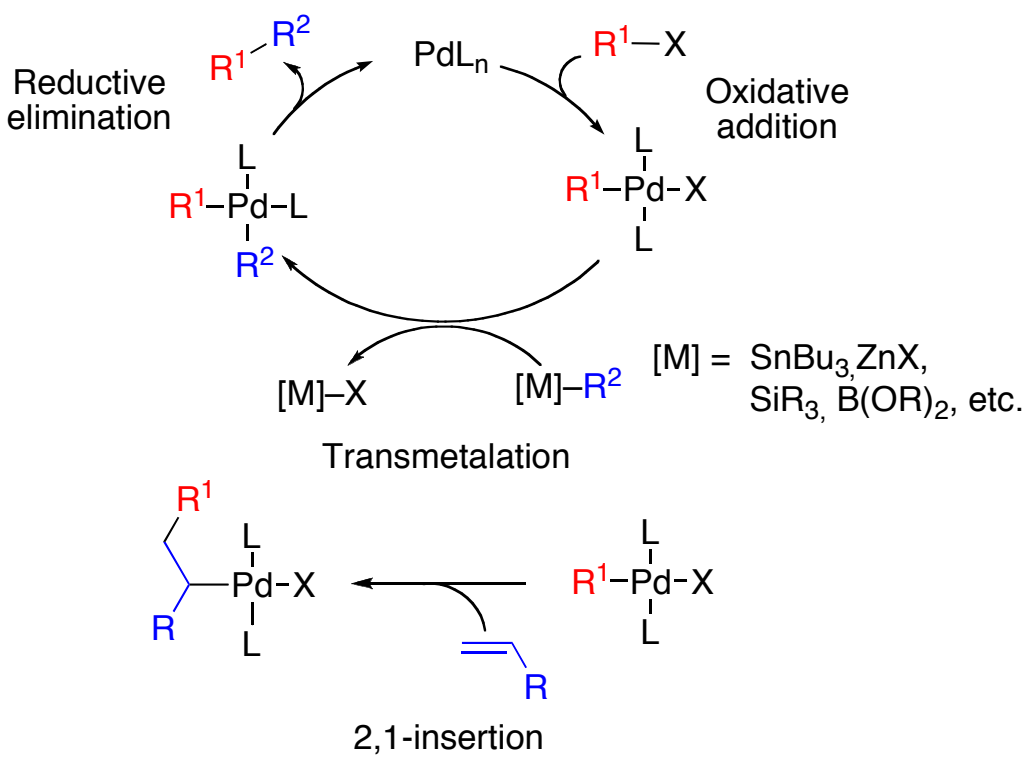


Figure 6. Simplified scheme for some of the elementary steps involved in Pd-catalyzed reactions.

\subsubsection{Oxidative addition and related processes}

\section{Oxidative addition to $P d(0)$ compounds}

The oxidative addition of perfluorinated substrates is a common step in different classes of palladium catalyzed reactions. The oxidative addition of perfluoroalkyl reagents adds to the difficulty of the inertness of the $\mathrm{C}\left(\mathrm{sp}^{3}\right)-\mathrm{X}$ bond, the low polarity of the $\mathrm{R}_{\mathrm{F}}-\mathrm{X}$ bond. The reactions are often performed under harsh conditions and radical intermediates have often been invoked to explain the results of the catalytic processes. No surprisingly, there are no detailed reports on the mechanism of the oxidative addition of fluoroalkyl halides, triflates or tosylates to palladium (0) complexes.

The oxidative addition of fluoroaryl halides is kinetically more favorable, and the addition of $\operatorname{Ar}_{\mathrm{F}}-\mathrm{I}$ to palladium(0) complexes is a well known procedure for the synthesis of perfluoroaryl palladium(II) complexes. Under catalytic conditions and nonfluorinated aryls, this reaction leads to trans-[PdArXL 2$]$ as the only observable product. However the use of fluorinated aryls allows the detection of other reaction intermediates. Thus, the oxidative addition of $\operatorname{Ar}_{\mathrm{F}} \mathrm{I}\left(\mathrm{Ar}_{\mathrm{F}}=3,5\right.$-dichloro-2,4,6trifluorophenyl) to $\left[\mathrm{Pd}\left(\mathrm{PPh}_{3}\right)_{4}\right]$ was shown to proceed in two steps, giving in a fast reaction $c i s-\left[\mathrm{PdAr}_{\mathrm{F}} \mathrm{IL}_{2}\right],\left(\mathrm{L}=\mathrm{PPh}_{3}\right)$, the actual product of oxidative addition, which then slowly isomerizes to the trans derivative. ${ }^{169}$ This supports a concerted, three-centered mechanism for the oxidative addition of aryl halides to $\operatorname{Pd}(0)$. The slow isomerization makes possible the isolation of the cis isomer cis-[ $\left.\operatorname{PdAr}_{\mathrm{F}} \mathrm{IL}_{2}\right]$, quite unusual in the chemistry of palladiun(II), and to study its isomerization process to the 
thermodynamically favored trans-[PdAr $\left.\mathrm{IL}_{2}\right]$. The process has been monitored by ${ }^{19} \mathrm{~F}$ NMR (Figure 7).

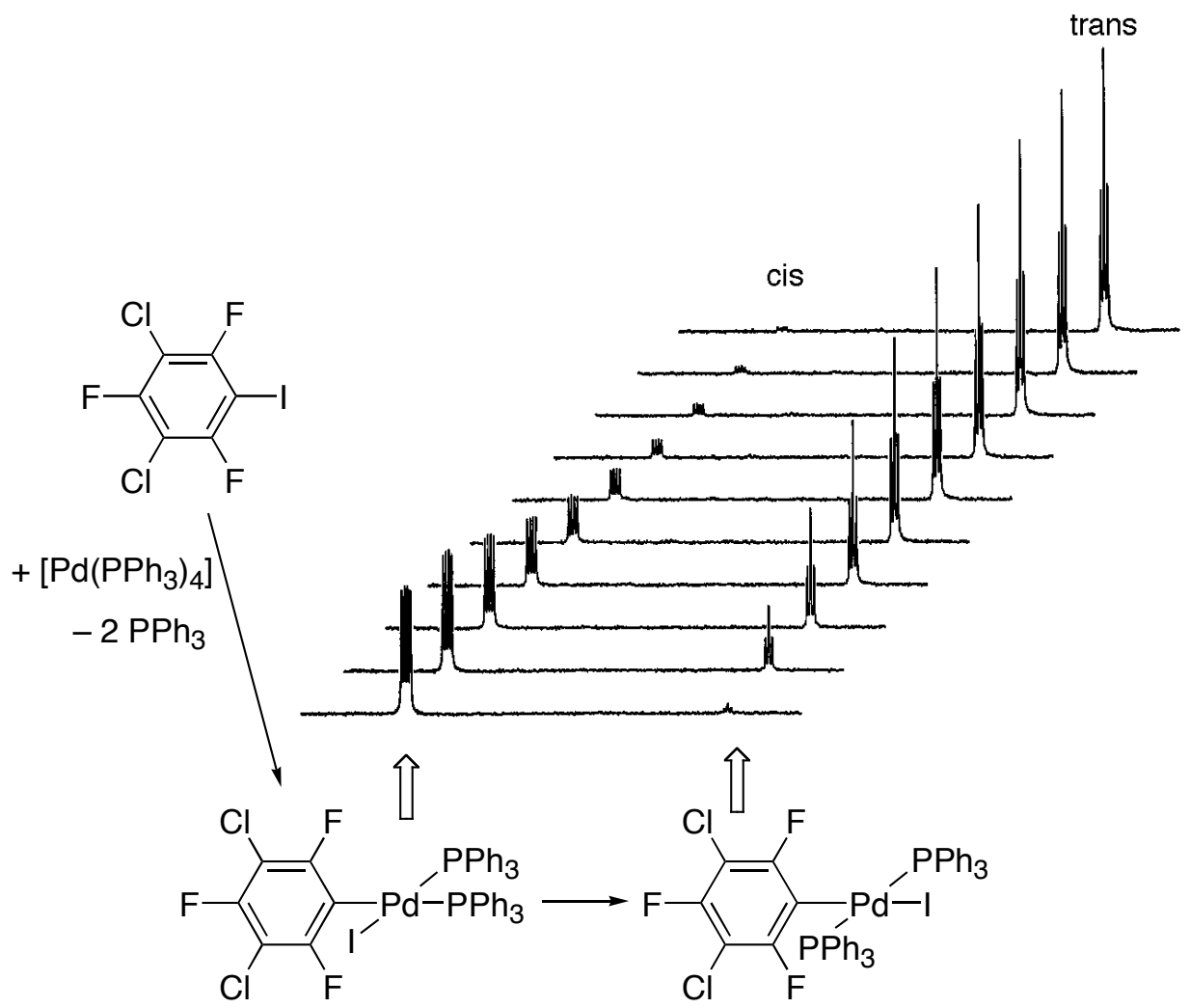

Figure 7. Isomerization of cis-[ $\left.\mathrm{PdAr}_{\mathrm{F}} \mathrm{IL}_{2}\right]$ formed by oxidative addition of $\mathrm{Ar}_{\mathrm{F}} \mathrm{I}$ to $\left[\mathrm{Pd}\left(\mathrm{PPh}_{3}\right)_{4}\right]$ as monitored by ${ }^{19} \mathrm{~F}$ NMR at 10 min intervals. Only the region of $\mathrm{F}_{\text {ortho }}$ in the complexes is shown. Under the reaction conditions the oxidative addition is fast and has been already completed in the first recording. Reproduced with permission from ref. 169 Copyright 1998, American Chemical Society.

The isomerization shows a double dependence on the concentration of the neutral ligand $\mathrm{PPh}_{3}$; at low concentration of $\mathrm{PPh}_{3}$ there is a strong retarding effect but this effect is very small above a certain threshold. In addition, the pseudo first order rate constant includes a second order dependence on the concentration of the complexes $\left[\operatorname{PdAr}_{\mathrm{F}} \mathrm{IL}_{2}\right]$. A complex four-pathway mechanism for the cis-trans isomerization is proposed consistent with these data, three of them are shown in Scheme 47, being the 
fourth one the Berry pseudorotation processes induced by the coordination of the solvent (THF) to the starting complex cis-[ $\left.\mathrm{PdAr}_{\mathrm{F}} \mathrm{IL}_{2}\right]$.

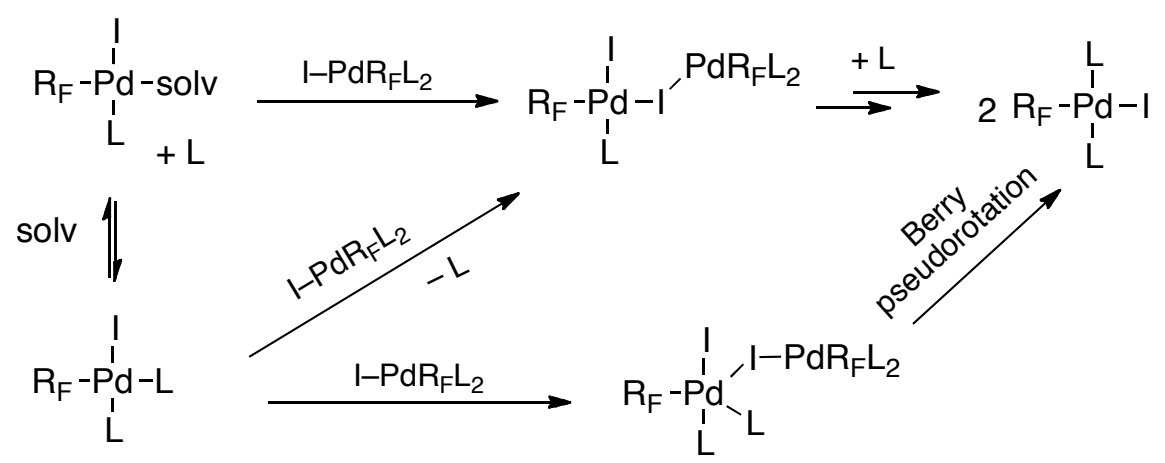

Scheme 47

As stated before, the trans isomer is usually found after the oxidative addition reaction when the isomerization step is faster than the addition reaction and this is the most common situation. During the oxidative addition of with $4^{\prime}$-chloro- $2^{\prime}, 3^{\prime}, 5^{\prime}, 6^{\prime}-$ tetrafluorostilbazole only the trans isomer is observed. ${ }^{170}$ The stilbazole can coordinate to palladium(0) through the double bound and the resulting palladium(0) complex has been isolated and has been characterized by X-ray diffraction. On this complex, the oxidative addition may progress by two pathways: i) by intramolecular migration of the palladium through the fluoroaromatic ring to reach the $\mathrm{C}-\mathrm{Cl}$ bond or ii) by dissociation of the stilbazole and a bimolecular reaction (Scheme 48). DFT calculations have shown that the second pathway is favored in the presence of phosphine $\left(\mathrm{PEt}_{3}\right)$ that is able to remove the coordinated olefin and generate the highly reactive intermediate $\left[\mathrm{Pd}\left(\mathrm{PEt}_{3}\right)_{3}\right]$. 


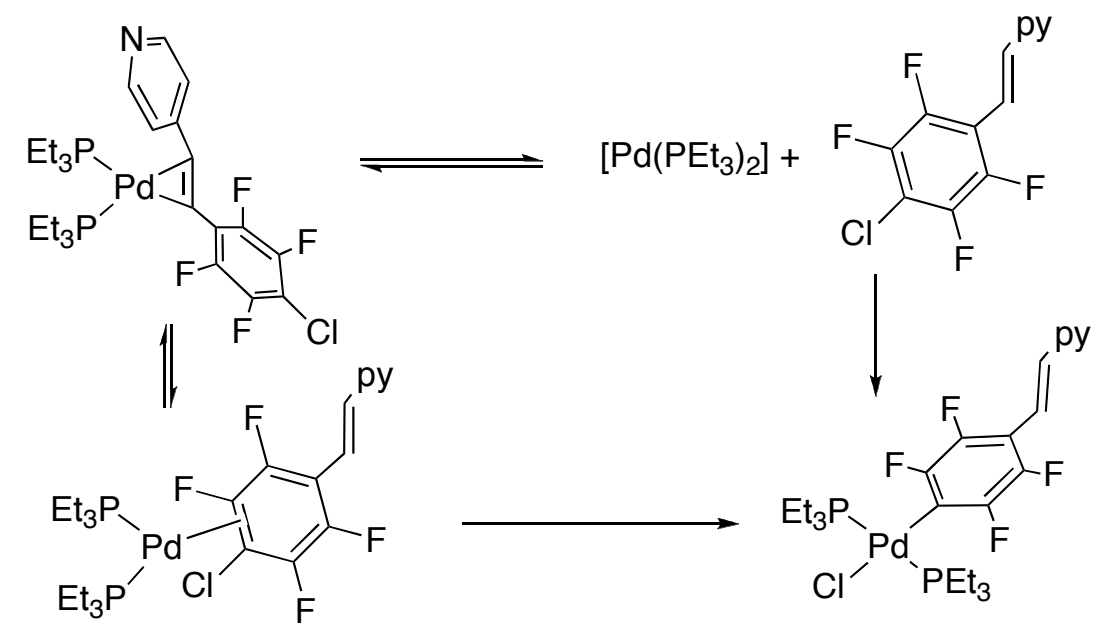

Scheme 48

Oxidation of Pd(II) compounds leading to organofluorine Pd(IV) derivatives

The oxidation of palladium(II) complexes to palladium(IV) with trifluoromethylating oxidants, such as Togni's and Unemoto's reagents, or with hipervalent iodine derivatives in the presence of trifluoromethylating reagens, is a key step in Pd(IV) catalyzed trifluoromethylation reactions. ${ }^{18,80,83}$ Ritter has proved that the product of oxidation of palladium(II) acetate complexes with $\mathrm{PhI}(\mathrm{OAc})_{2}$ is, in fact, a palladium(III) dimer (Scheme 49, a), and that the reductive elimination of aryl acetates takes place easier from a $\operatorname{Pd}(\mathrm{III})$ than from a $\mathrm{Pd}(\mathrm{IV})$ intermediates. ${ }^{171}$ Thus, the formation of palladium(III) dimeric complexes provides a low energy pathway in redox processes involving the formation of C-X bonds. ${ }^{172}$ In contrast, Sanford et al. reported in 2010 that the treatment of the binuclear complex shown in Scheme 49 b with Togni's reagent affords a mononuclear trifluoromethyl Pd(IV) complex (Scheme 49, b). ${ }^{173}$ In the same work, oxidation of trifluoromethyl palladium(II) complex $\left[\mathrm{Pd}\left(\mathrm{CF}_{3}\right)(2-\right.$ $\left.\left.\mathrm{PhC}_{6} \mathrm{H}_{4}\right)(\mathrm{dtbpy})\right]$ with $\mathrm{PhICl}_{2}$ produces cis- $\left[\mathrm{Pd}\left(\mathrm{CF}_{3}\right)\left(2-\mathrm{PhC}_{6} \mathrm{H}_{4}\right) \mathrm{Cl}_{2}(\mathrm{dtbpy})\right]$ without any observable intermediate, ${ }^{174}$ suggesting a different behavior when the trifluoromethyl group is involved in the reaction. 
(a)

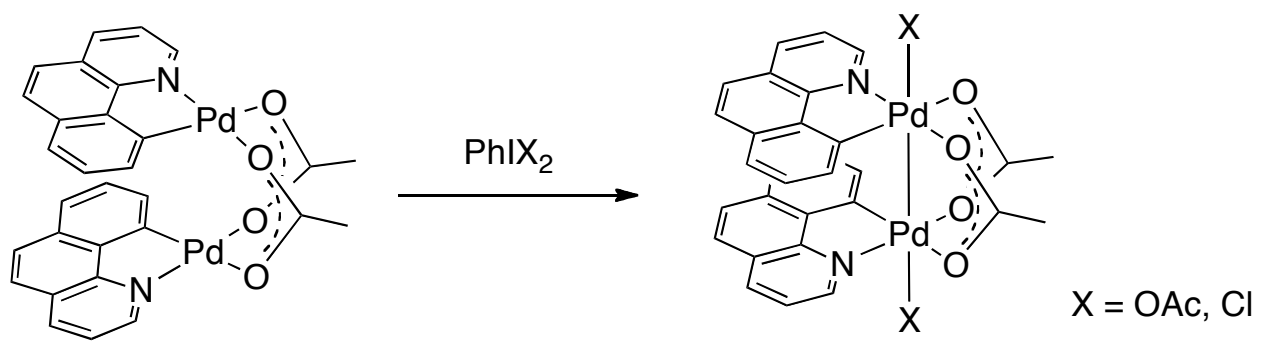

(b)
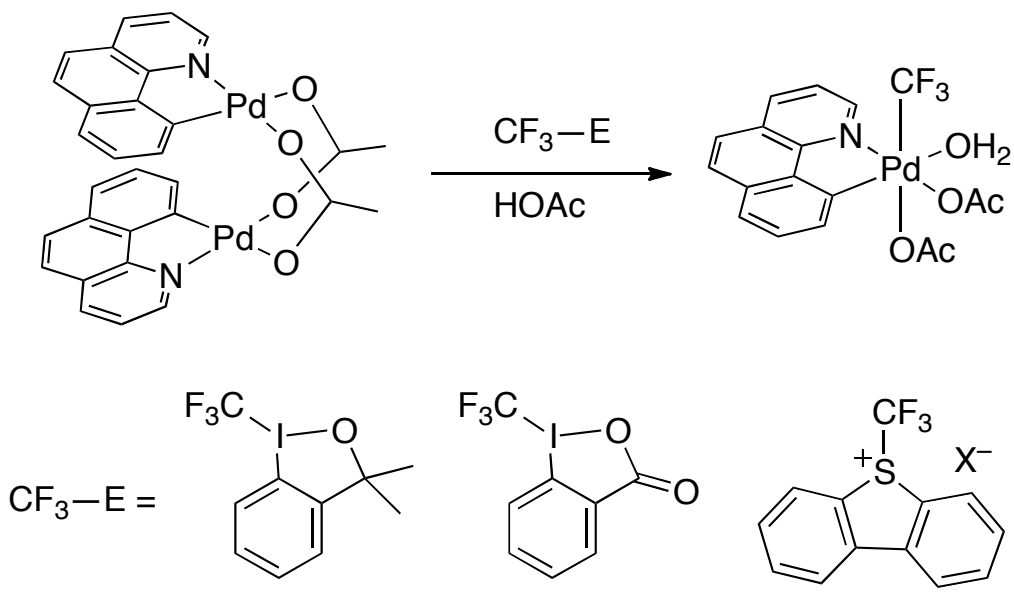

Scheme 49

In a elegant work the groups of Ritter and Sanford addressed this problem by a kinetic study and DFT calculations on the oxidation of Pd(II) acetate complexes with Togni's reagent and also on the oxidation with other hipervalent iodine reagents and reaction with a $\mathrm{CF}_{3}^{-}$source, typically the $\mathrm{CF}_{3} \mathrm{SiMe}_{3} / \mathrm{CsF}$ system. They found that starting from the dinuclear $[\mathrm{Pd}(\mu-\mathrm{OAc})(\text { benzoquinolinato })]_{2}$ complex, the kinetics of oxidation with Togni's reagent follow the rate law $\mathrm{r}=\mathrm{k}[\mathrm{Pd}(\mathrm{II})][$ oxidant $][\mathrm{AcOH}]$, consistent with the oxidation of the dimeric species with the protonated Togni's reagent. This rate law suggests the formation of a dimeric trifluoromethyl intermediate as shown in Scheme 50 (a), which subsequently, in the presence of acetate, splits into the observed $\mathrm{Pd}(\mathrm{IV})$ complex and a $\mathrm{Pd}(\mathrm{II})$ acetate complex which after dimerization can reenter the oxidation process. In addition, they were able to trifluoromethylate a $\mathrm{Pd}(\mathrm{III})$ 
complex produced by oxidation of the $\mathrm{Pd}(\mathrm{II})$ starting material with $\mathrm{XeF}_{2}$. The reaction of this complex with $\mathrm{HAcO}$, produces the hydrolysis of one of the trifluoromethyl groups and a fast decomposition of the intermediate in a $\mathrm{Pd}(\mathrm{IV})$ trifluoromethyl derivative and the $\mathrm{Pd}(\mathrm{II})$ complex (Scheme 50, b).

a)

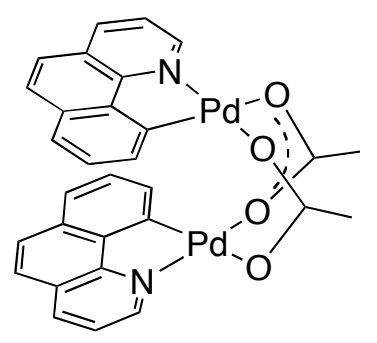

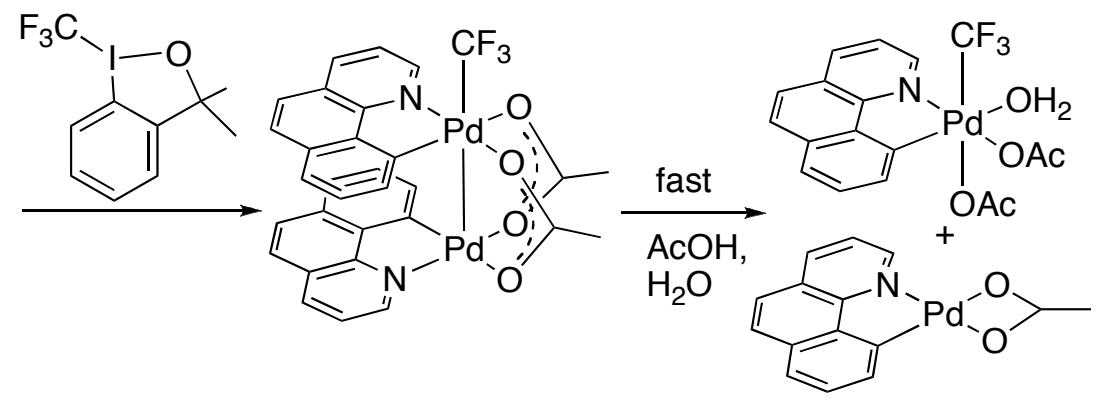

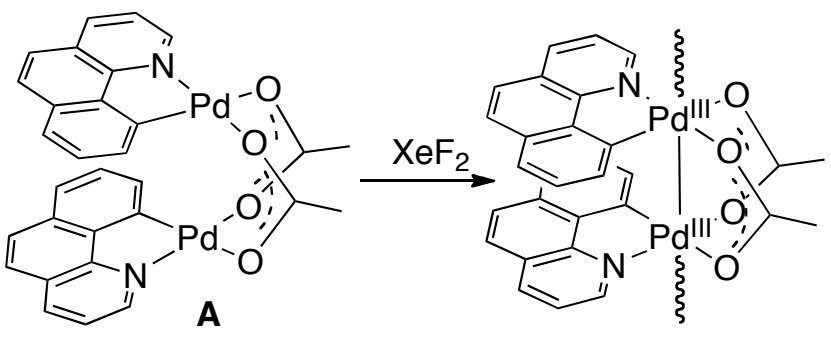

A

b)

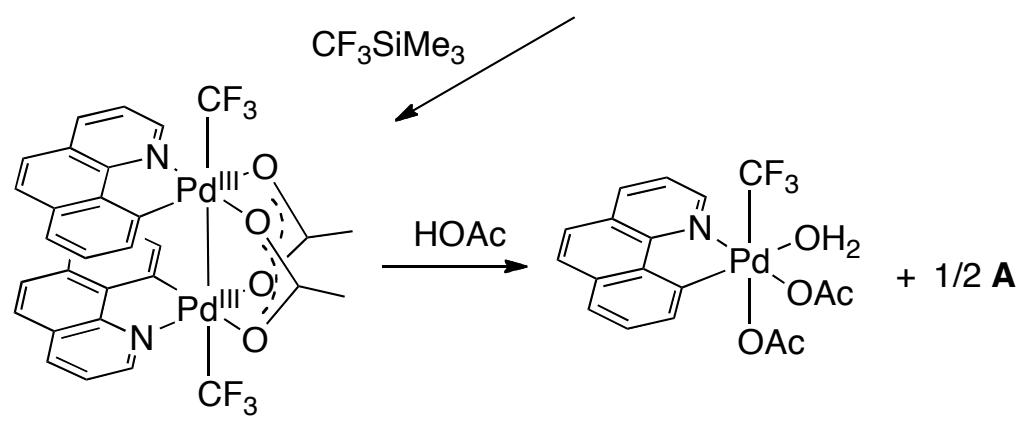

Scheme 50

Although the possibility of direct oxidation processes of palladium(II) complexes to palladium(IV) cannot be ruled out, particularly for those complexes with ligands that are unable to produce dimeric structures, the results of this work strongly support a two-step, oxidation-disproportionation sequence for the formation of trifluoromethyl palladium(IV) intermediates when using palladium acetate derivatives. 


\subsubsection{Transmetalation}

Transmetalation is the step that produces the exchange of organic groups between metals (Scheme 51) and then it is a critical step in any reaction in which a M$\mathrm{R}_{\mathrm{F}}$ bond is created in one active metal (i.e. zinc, tin, etc.) and the $\mathrm{R}_{\mathrm{F}}$ group needs to be transferred to palladium in order to get a controlled reactivity (i.e. a C-C bond formation via reductive elimination). There are several catalytic processes in which this step plays a role, ${ }^{38,83}$ such as the Stille and the Negishi cross-couplings, and also coupling reactions involving silicon and copper trifluoromethyl reagents.

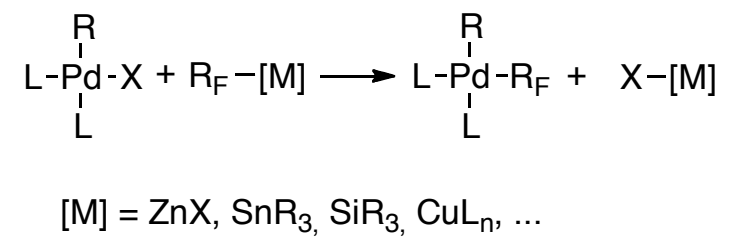

Scheme 51

Transmetalation processes can take place between palladium complexes, provided they are stable enough not to decompose by reductive elimination. The exchange of different fluoroaryl groups (pentafluorophenyl and 3,5-dichloro,2,4,6trifluorophenyl) has been studied by ${ }^{19} \mathrm{~F}$ NMR. A mechanism is proposed which is consistent with the observed conservation of the stereochemistry of the complexes $\left(\right.$ Scheme 52). ${ }^{175}$

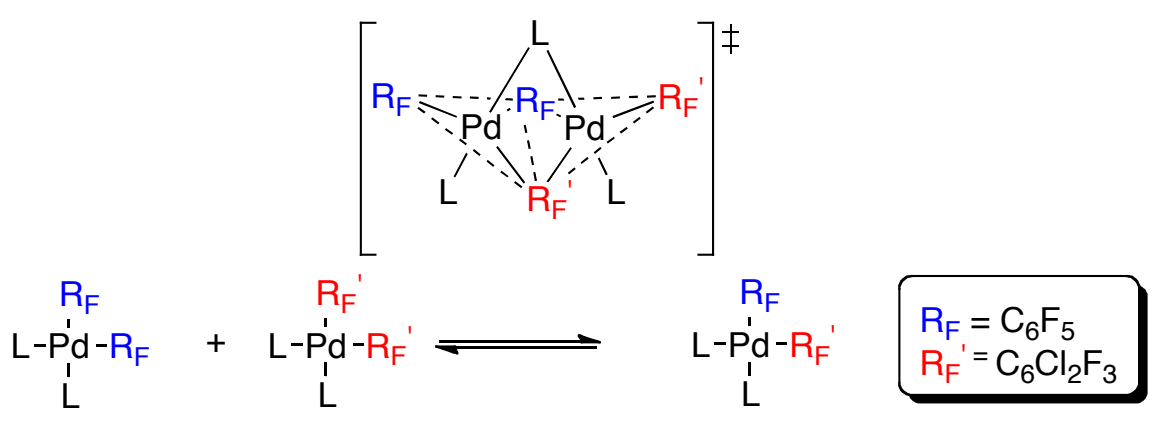

Scheme 52 
The transmetalation step in the Stille reaction.

The use of fluorinated aryls has been particularly useful in the study of transmetalation processes related to the Stille cross-coupling reaction, and the conclusions reached with the use of fluorinated aryls exceeds the organofluorine chemistry and are of general application. This reaction is the substitution of a halide or pseudohalide coordinated to the palladium atom by one aryl or vinyl group from an organotin derivative, but very often it is not a single-step reaction, but a complex sequence of reactions. Because of this complexity, the stereochemistry of the substitution at the carbon and at the palladium centers and the kinetic effects of the ligands or other additives not always have a straightforward explanation.

The mechanism has been studied in depth for the cross coupling of 3,5-dichloro2,4,6-trifluorophenyl iodide with vinyl- or $p$-methoxy-phenyltributyltin (Scheme 53). ${ }^{103}$

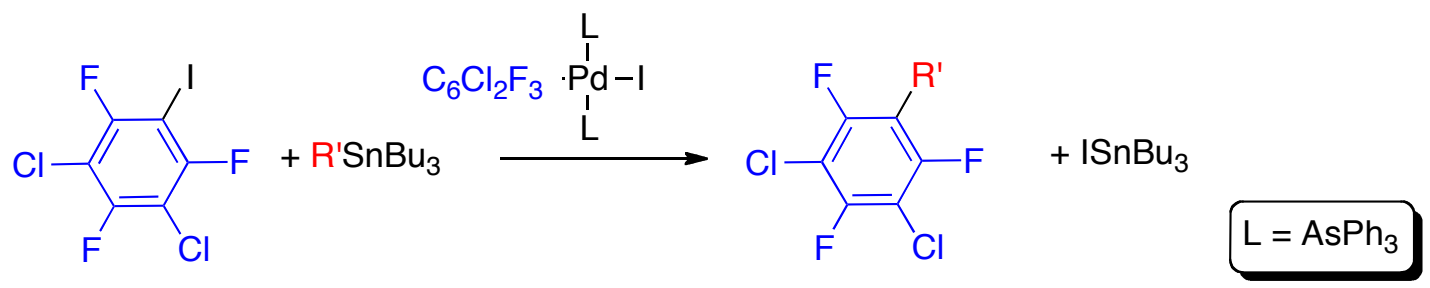

Scheme 53

In this reaction the catalyst is the complex $\left[\mathrm{Pd}\left(\mathrm{C}_{6} \mathrm{Cl}_{2} \mathrm{~F}_{3}\right) \mathrm{I}\left(\mathrm{AsPh}_{3}\right)_{2}\right]$, which in turn can be obtained by oxidative addition of $\mathrm{C}_{6} \mathrm{Cl}_{2} \mathrm{~F}_{3} \mathrm{I}$ to $\mathrm{Pd}_{2}(\mathrm{dba})_{3} \cdot \mathrm{CHCl}_{3}$ in the presence of triphenylarshine. Hence, the transmetalation step can be studied on the isolated paladium(II) complexes. The kinetics of the reaction show a minus one order dependence of the reaction rate on the concentration of ligand, so the substitution of the arsine by the weak nucleophile $\mathrm{R}^{\prime} \mathrm{SnBu}_{3}$ is proposed to be a previous step to the rate limiting transmetalation (Scheme 54). 


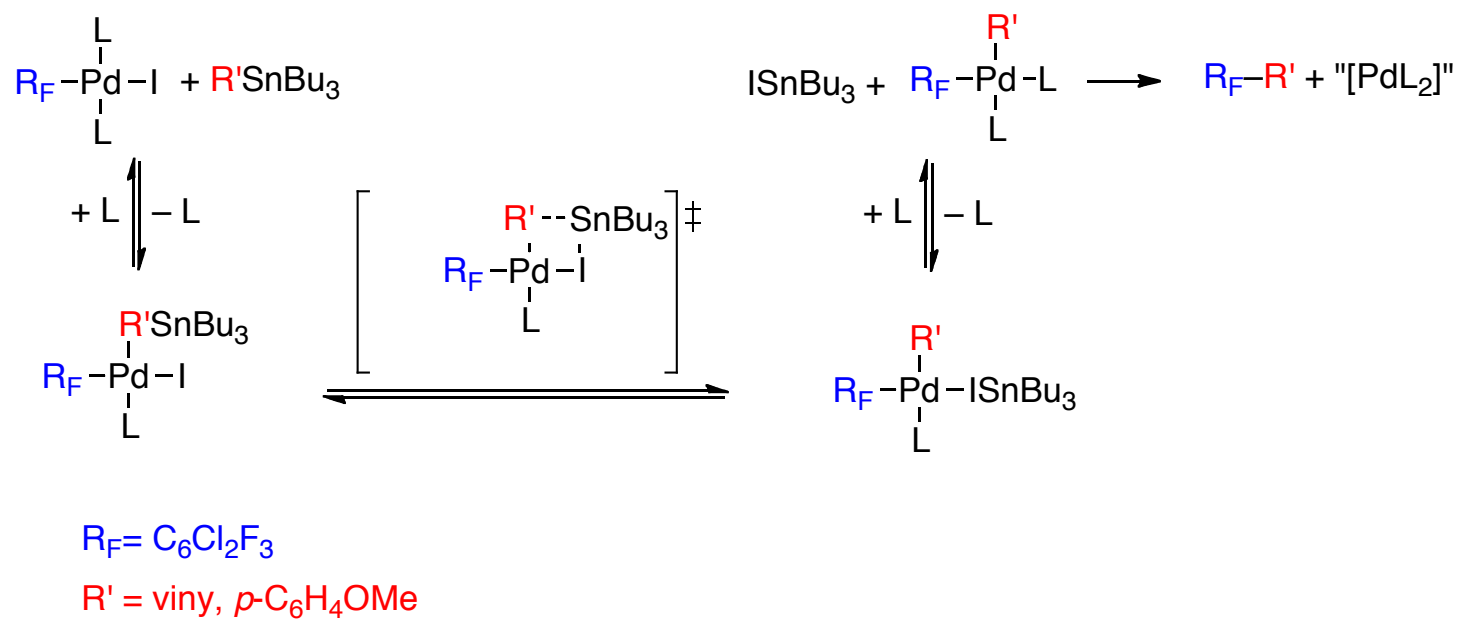

Scheme 54

Following this pathway, the product of the transmetalation is the cis isomer, from which the reductive elimination can take place immediately without the requirement of further isomerization. It is this last step, the reductive elimination, which drives the reaction, being the $\mathrm{C}-\mathrm{C}$ bond forming $\Delta \mathrm{G}$ the thermodynamic driving force. In fact, the transmetalation of fluorinated aryls from organotin fragments to aryl palladium(II) complexes is energetically uphill, and probably this is one of the reasons why the use of the Stille reaction for the synthesis of fluoroaryl derivatives is not as common as for other nonfluorinated groups.

Taking advantage of the high kinetic stability of the fluoroaryl complex cis$\left[\mathrm{Pd}\left(\mathrm{C}_{6} \mathrm{Cl}_{2} \mathrm{~F}_{3}\right)_{2}\left(\mathrm{AsPh}_{3}\right)_{2}\right]$, the study of the reversal to the transmetalation reaction (the retrotransmetalation) with $\mathrm{SnBu}_{3} \mathrm{I}$ to produce trans- $\left[\mathrm{Pd}\left(\mathrm{C}_{6} \mathrm{Cl}_{2} \mathrm{~F}_{3}\right) \mathrm{I}\left(\mathrm{AsPh}_{3}\right)_{2}\right]$ and $\mathrm{SnBu}_{3} \mathrm{C}_{6} \mathrm{Cl}_{2} \mathrm{~F}_{3}$. The intermediate cis- $\left[\mathrm{Pd}\left(\mathrm{C}_{6} \mathrm{Cl}_{2} \mathrm{~F}_{3}\right)_{2}\left(\mathrm{AsPh}_{3}\right)\left(\mathrm{SnBu}_{3} \mathrm{I}\right)\right]$ has been detected by ${ }^{19} \mathrm{~F}$ NMR in the course of the reaction. DFT calculations support a concerted transitions state in which the $\mathrm{Sn}-\mathrm{I}$ and $\mathrm{Pd}-\mathrm{C}$ bond, and $\mathrm{Pd}-\mathrm{I}$ and $\mathrm{Sn}-\mathrm{C}$ a are been created and broken simultaneously (Figure 8). ${ }^{176}$ 

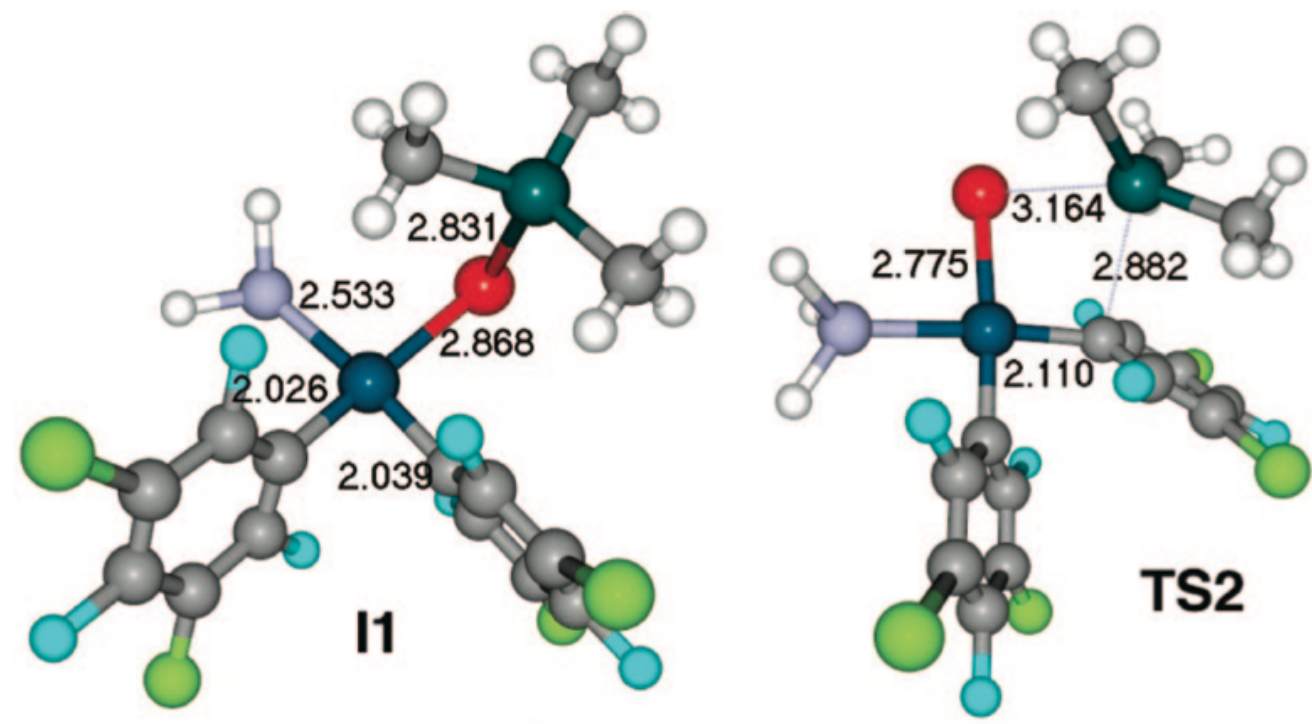

Figure 8. DFT calculated structures for the intermediate on the retrotransmetalation reaction $\left[\mathrm{Pd}\left(\mathrm{C}_{6} \mathrm{Cl}_{2} \mathrm{~F}_{3}\right)_{2}\left(\mathrm{ISnMe}_{3}\right)\left(\mathrm{AsH}_{3}\right)\right]$ (left) and the transition state of the transmetalation (right). Taken from reference. Reproduced with permission from ref. 176. Copyright 2008, American Chemical Society.

Although the cross coupling of $\mathrm{C}_{6} \mathrm{~F}_{5} \mathrm{I}$ or $\mathrm{C}_{6} \mathrm{Cl}_{2} \mathrm{~F}_{3} \mathrm{I}$ with aryl or vinyl tributyltin takes place effectively under the conditions described in reference 103 that use $\mathrm{AsPh}_{3}$ as ligand, the reaction performed with $\mathrm{PPh}_{3}$ is very slow, but is much more efficient with the aid of copper iodide as co-catalyst. The main role of the copper in this system is to remove ligand from the reaction medium, forming the known complexes $\left[\mathrm{Cu}\left(\mathrm{PPh}_{3}\right)_{\mathrm{n}}\right]$, and facilitating the nucleophilic attack of the organotin to the palladium center. Since the affinity of copper(I) for arsine ligads is much lower that for phosphine, when $\mathrm{AsPh}_{3}$ is used as ligand the addition of $\mathrm{CuI}$ has no effect on the reaction rate. ${ }^{177}$

In the Stille reaction the transmetalation usually takes place on the trans isomer $\left[\mathrm{PdRXL}_{2}\right]$ that is formed after the oxidative addition of the aryl halide $\mathrm{R}-\mathrm{X}$ to the palladium (0) intermediate followed by a fast isomerization. However, if the aryl halide is a polyfluorinated aryl such $\mathrm{C}_{6} \mathrm{~F}_{5} \mathrm{I}$ or $\mathrm{C}_{6} \mathrm{Cl}_{2} \mathrm{~F}_{3} \mathrm{I}$, the isomerization cis- to -trans- 
$\left[\mathrm{PdR}_{\mathrm{F}} \mathrm{IL}_{2}\right]$ is a slow reaction at room temperature and both isomers can be isolated and characterized. ${ }^{169}$ Under the conditions of the Stille cross-coupling, this circumstance produces in solution a mixture of isomers cis- and trans- $\left[\mathrm{PdR}_{\mathrm{F}} \mathrm{IL}_{2}\right]$ that can undergo the transmetalation reaction competitively. ${ }^{104}$ The reaction has been studied for the cross coupling of $\mathrm{C}_{6} \mathrm{Cl}_{2} \mathrm{~F}_{3} \mathrm{I}$ with phenylethynyltributyltin. The transmetalation of the alkynyltin to each one of the isomers $\left[\operatorname{Pd}\left(\mathrm{C}_{6} \mathrm{Cl}_{2} \mathrm{~F}_{3}\right) \mathrm{IL}_{2}\right]\left(\mathrm{L}=\mathrm{PPh}_{3}\right)$ has been independently studied and it was found that the reaction is not regioselective at the palladium. Thus a mixture of cis and trans- $\left[\mathrm{Pd}\left(\mathrm{C}_{6} \mathrm{Cl}_{2} \mathrm{~F}_{3}\right)(\mathrm{CCPh}) \mathrm{L}_{2}\right]$ complexes is obtained regardless the isomer that participates in the transmetalation (Scheme 55). The very slow isomerization of the trans- $\left[\mathrm{Pd}\left(\mathrm{C}_{6} \mathrm{Cl}_{2} \mathrm{~F}_{3}\right)(\mathrm{CCPh}) \mathrm{L}_{2}\right]$ and its inability to give the cross coupling product by reductive elimination, results in its accumulation in solution. Hence, in this system the amount of palladium that is able to participate in the catalysis decreases steadily during the reaction course.

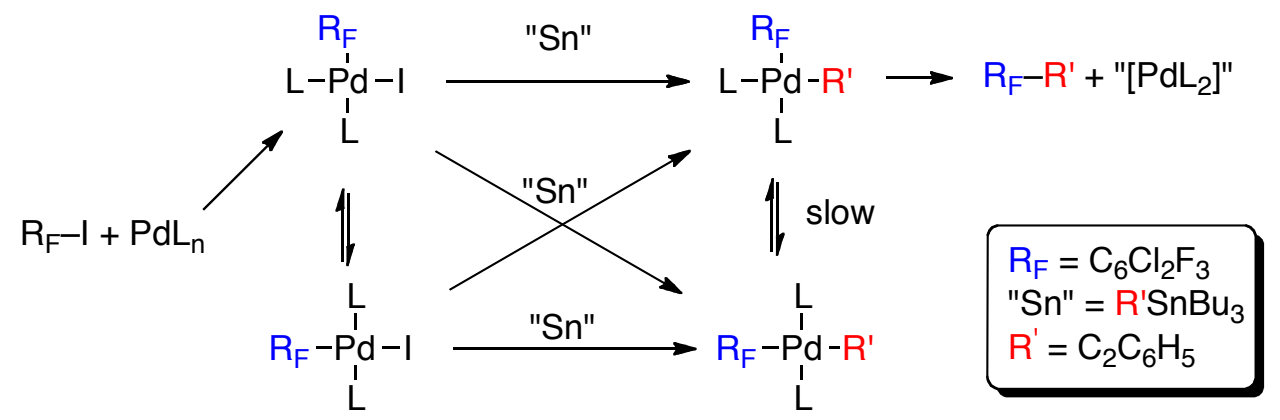

Scheme 55

When the transmetalation takes place on complexes bearing triflate instead of halides, or when the reaction proceeds in a strong coordinating solvents, the course of the reaction changes, and the removal of the weakest ligand by the organotin reagent can be produced through an open transition state (Scheme 56) ${ }^{178}$ By this route the 
transmetalation to trans- $\left[\mathrm{PdR}_{\mathrm{F}} \mathrm{XL}_{2}\right]$ gives the trans- $\left[\mathrm{PdR}_{\mathrm{F}} \mathrm{R}^{\prime} \mathrm{L}_{2}\right]$ isomer. For $\mathrm{L}=\mathrm{PPh}_{3}$, $\mathrm{R}_{\mathrm{F}}=\mathrm{C}_{6} \mathrm{Cl}_{2} \mathrm{~F}_{3}$ and $\mathrm{R}^{\prime}=$ vinyl, this isomer has been detected in solution, reinforcing the mechanistic proposal. This dual transmetalation pathway, open (Scheme 56) and cyclic (Scheme 54) transition states, provides a satisfactory explanation for the observed lack of selectivity during the transmetalation for the abovementioned alkynyltin derivatives, which can follow the cyclic pathway but, being good ligands and thus capable of removing iodine from the coordination sphere of the palladium, could also follow the open pathway. More importantly the dual transmetalation pathway explains the retention and inversion of the stereochemistry at the carbon observed for different enantiopure organotin derivatives under different reaction conditions. ${ }^{179}$

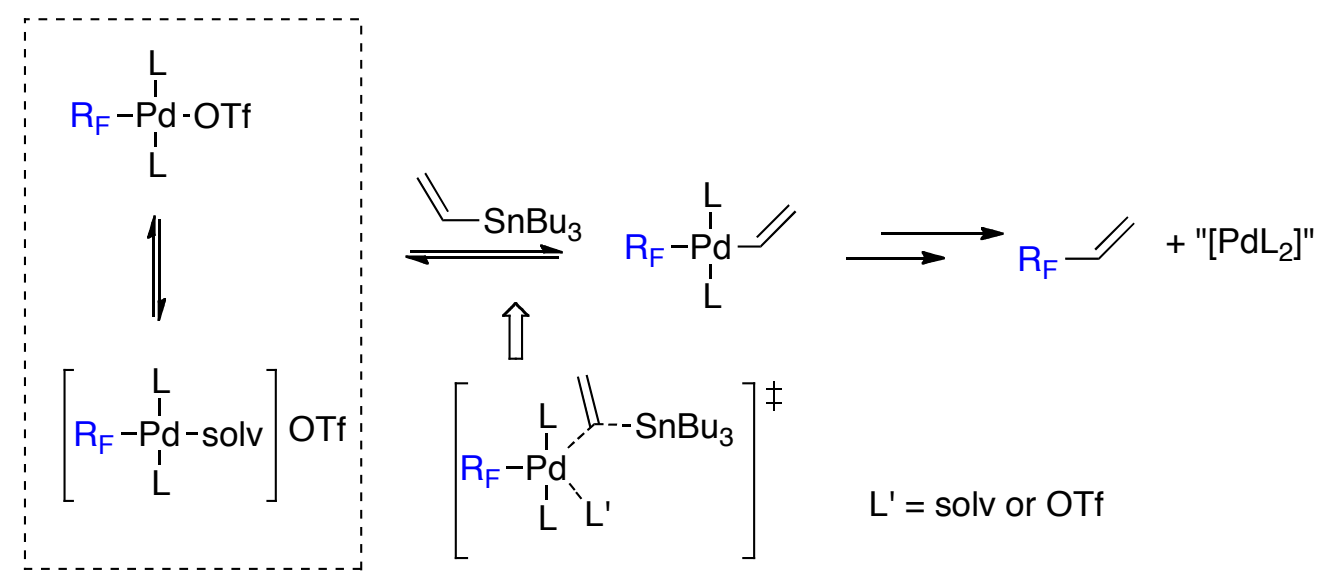

Scheme 56.

The very slow reductive elimination rate of fluoroaryl derivatives has also allowed the detection of complexes $\left[\mathrm{PdR}_{\mathrm{F}}(\operatorname{vinyl})(\mathrm{dppe})\right]\left(\mathrm{R}_{\mathrm{F}}=\mathrm{C}_{6} \mathrm{~F}_{5}, \mathrm{C}_{6} \mathrm{Cl}_{2} \mathrm{~F}_{3}\right.$; dppe $=$ 1,2-bis(diphenylphosphino)ethane), formed by transmetalation of $\mathrm{SnBu}_{3}($ vinyl), in which the aryl and the vinyl group occupies a cis position. ${ }^{180}$ 
Transmetalation equilibria between organogold and organopalladium complexes.

The catalytic effect of fluoroaryl gold(I) complexes in the trans-cis isomerization of trans-[PdR $\left.\mathrm{Pd}_{2} \mathrm{~L}_{2}\right]\left(\mathrm{R}_{\mathrm{F}}=\mathrm{C}_{6} \mathrm{~F}_{5}, \mathrm{C}_{6} \mathrm{Cl}_{2} \mathrm{~F}_{3} ; \mathrm{L}=\right.$ tetrahydrotiophene $)$ complexes was reported in $1998 .^{181}$ This reaction involves the aryl transfer between gold and palladium, and the process is related to the transmetalation processes involved in the gold-palladium cocatalyzed Stille reaction. ${ }^{182,183}$ The transarylation from cis$\left[\mathrm{Pd}\left(\mathrm{R}_{\mathrm{F}}\right)_{2} \mathrm{~L}_{2}\right]$ to $[\mathrm{AuClL}]\left(\mathrm{R}_{\mathrm{F}}=3,5\right.$-dichloro,2,4,6-trifluorofenil; $\mathrm{L}=$ triphenylarsine $)$ shown in Scheme 57 has been studied. The kinetics of the reaction resembles those found for the retrotransmetalation with organotin derivatives: The reaction proceeds through the substitution of one neutral ligand followed by the transarylation from palladium to gold (Scheme 57). Also, as it is the case of the organotin derivatives, the equilibrium is shifted towards the formation of the monoarylated palladiun(II) complex. ${ }^{184}$
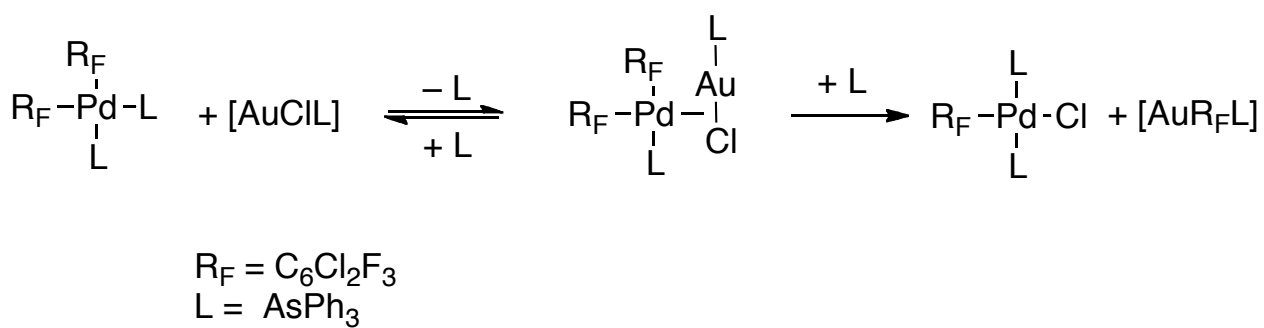

Scheme 57

However, DFT calculations shown that similarities are more formal than real, and that the interaction of palladium(II) with gold and tin have very few common features. To begin with, the replacement of the neutral ligand on palladium by the incoming metal halide is produced by the halogen atom when $\mathrm{ISnBu}_{3}$ is used (see Scheme 54 and Figure 8). The formation of the analogous intermediate with [AuClL] coordinated to the palladium through the chlorine is disfavored, and instead a gold 
initiated interaction leads to a intermediate with a short distance between gold and palladium (Figures 9 and 10, left). Also, the transition state is different. In both transmetalations the mechanism is a concerted cleavage of $\mathrm{Pd}-\mathrm{C}$ and $\mathrm{M}-\mathrm{X}$ bonds and the formation of $\mathrm{Pd}-\mathrm{X}$ and $\mathrm{M}-\mathrm{C}$ bonds $(\mathrm{M}=\mathrm{Sn}$ or $\mathrm{Au}, \mathrm{X}=\mathrm{I}$ or $\mathrm{Cl})$. However, the transmetalation to gold proceeds through the metalophilic interaction between gold and palladium, with a distance between metals close to the range of covalent Pd-Au bonds. In a recent report of the gold-palladium co-catalyzed Stille reaction, the transmetalation of $[\mathrm{AuRL}]$ and $\mathrm{RSnMe}_{3}$ to $\left[\mathrm{PdR}^{\prime} \mathrm{ClL}_{2}\right]$ have been calculated, reaching the same conclusions about the mechanism and the geometry of the transition state. ${ }^{182}$

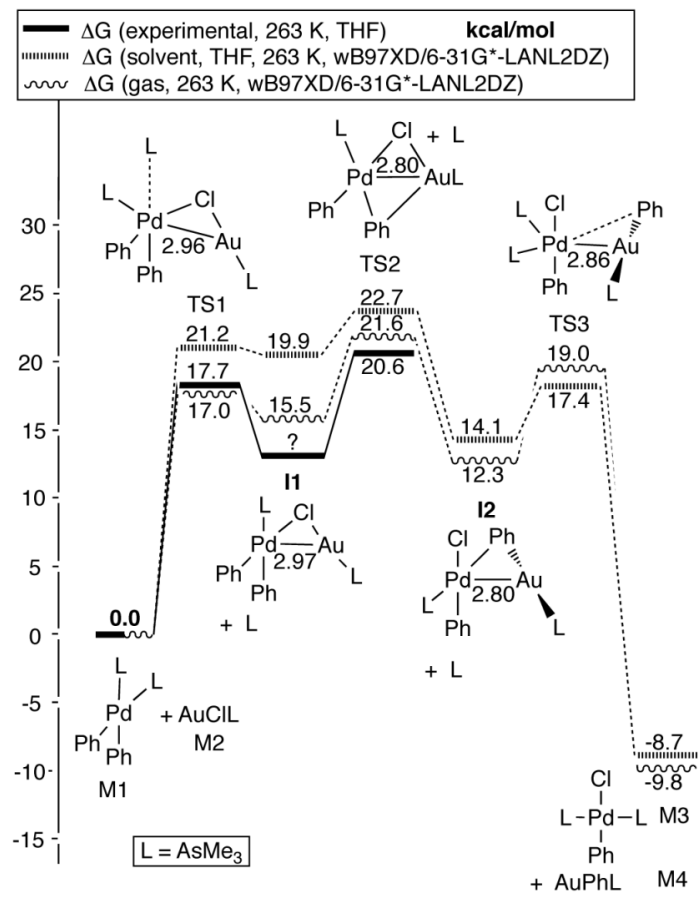

Figure 9. Calculated (DFT) and experimental reaction profile for the transmetalation from cis- $\left[\mathrm{PdR}_{\mathrm{F} 2} \mathrm{~L}_{2}\right]$ to $[\mathrm{AuClL}]$. Reproduced with permission from ref. 184. Copyright 2012, John Wiley\&Sons. 

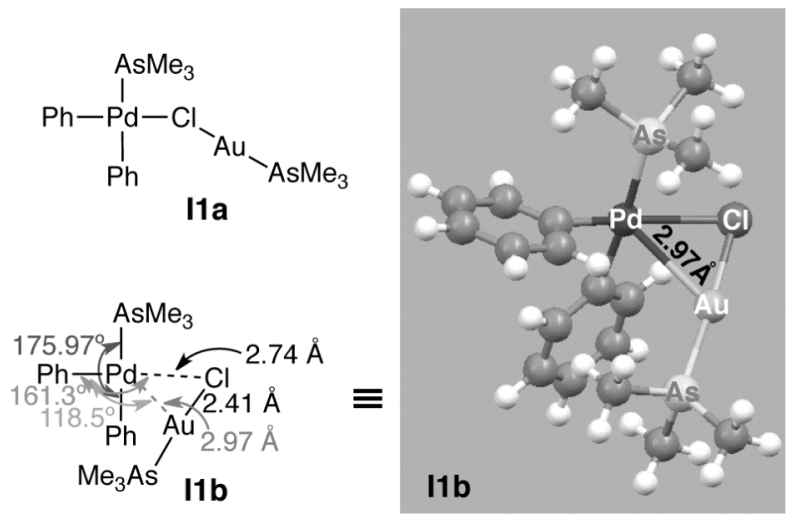

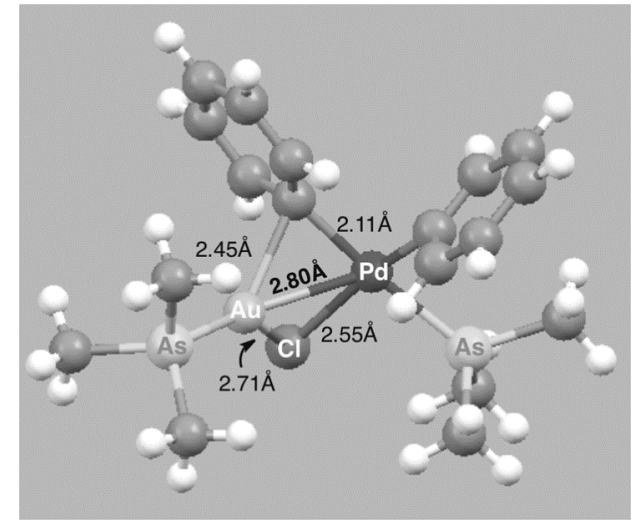

Figure 10. Left: Calculated structures for the intermediates in which the gold complex has substituted one arsine ligand. Intermediate I1a in which the substitution is produced by the halide exclusively is discarded (ref 184). Right: Calculated structure for the transition state for the transmetalation step. Reproduced with permission from ref. 184. Copyright 2012, John Wiley\&Sons.

The transmetalation step in the Negishi reaction.

The complex $\left[\mathrm{PdR}_{\mathrm{F}} \mathrm{Cl}\left(\mathrm{PPh}_{3}\right)_{2}\right]\left(\mathrm{R}_{\mathrm{F}}=\mathrm{C}_{6} \mathrm{Cl}_{2} \mathrm{~F}_{3}\right)$ catalyze the Negishi crosscoupling of $\mathrm{ZnMe}_{2}$ or $\mathrm{ZnMeCl}$ with $\mathrm{C}_{6} \mathrm{Cl}_{2} \mathrm{~F}_{3}$ I. These reactions are unique examples of palladium catalyzed cross-coupling of $\mathrm{C}\left(\mathrm{sp}^{3}\right)$ with a fluoroaryl group, being both quite inert towards the reductive elimination process. ${ }^{185}$ The transmetalation step is faster than the reductive elimination, and can be spectroscopically studied as a separate reaction. Also, the products of the transmetalation, cis- and trans-[PdR $\left.\mathrm{Fe}\left(\mathrm{PPh}_{3}\right)_{2}\right]$ are isolable.

The most dazzling feature of this reaction is the perhaloaryl group exchange between palladium and zinc, that takes place at a fast rate during the transmetalation producing $\mathrm{ZnR}_{\mathrm{F}} \mathrm{Me}$ or $\mathrm{ZnR}_{\mathrm{F}} \mathrm{Cl}$ depending on the organozinc used for the catalysis (Figure 11). Although a retro-transmetalation process, analogous to that reported above for ISnBu3 or the gold complex [AuClL] cannot be discarded, ${ }^{186}$ a direct exchange of the aryl group by the methyl operating in the complexes $\left[\mathrm{PdR}_{\mathrm{F}} \mathrm{MeL}_{2}\right]$ seems more plausible under the reaction conditions used (a large excess of $\mathrm{ZnMe}_{2}$ or $\left.\mathrm{ZnMeCl}\right){ }^{187,188}$ 


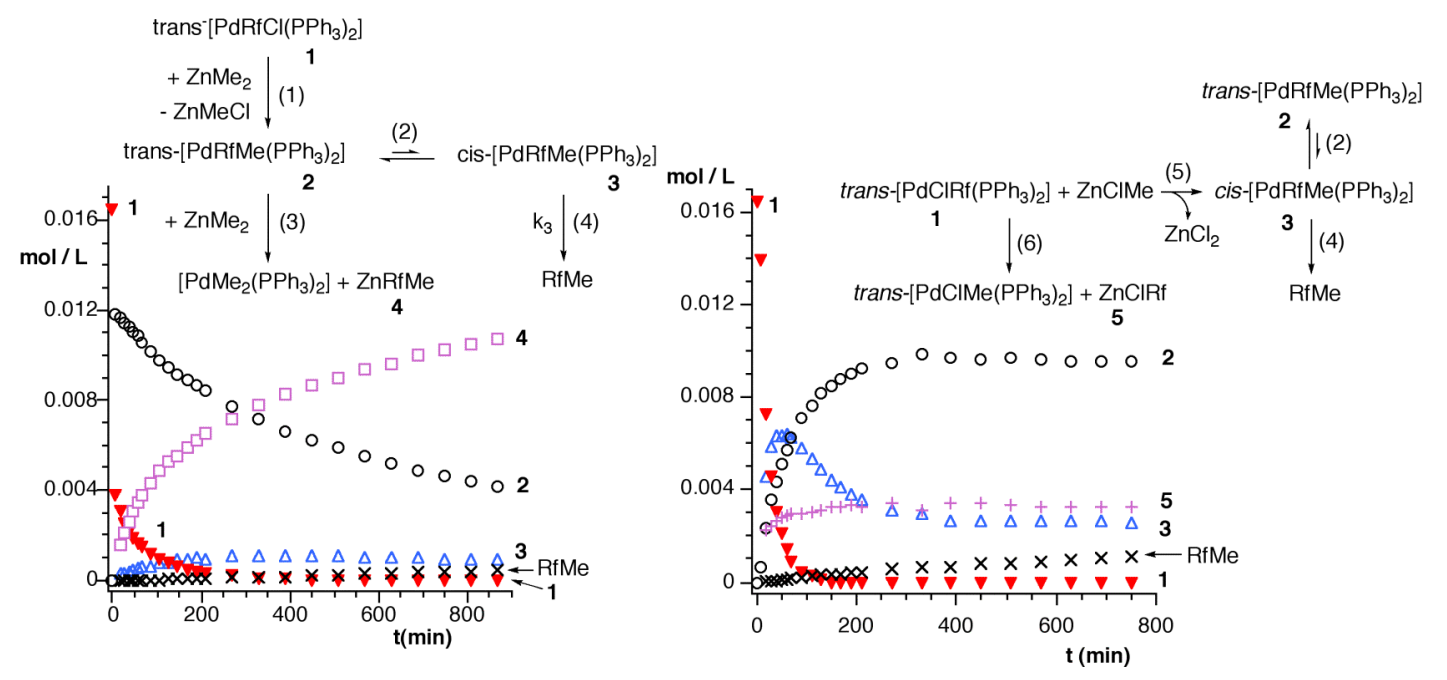

Figure 11. Concentration/time data for the reaction $\left[\mathrm{PdR}_{\mathrm{F}} \mathrm{Cl}\left(\mathrm{PPh}_{3}\right)_{2}\right]+\mathrm{ZnMe}_{2}$ (left) and $\left[\mathrm{PdR}_{\mathrm{F}} \mathrm{Cl}\left(\mathrm{PPh}_{3}\right)_{2}\right]+\mathrm{ZnMeCl}$ (right) obtained by ${ }^{19} \mathrm{~F} \mathrm{NMR}$, in THF at $298 \mathrm{~K}$. Note the formation of the transarylation products $\mathrm{ZnR}_{\mathrm{F}} \mathrm{Me}$ and $\mathrm{ZnR}_{\mathrm{F}} \mathrm{Cl}$, and the different rates or formation of trans and cis-[PdR $\left.\mathrm{P} \mathrm{Me}\left(\mathrm{PPh}_{3}\right)_{2}\right]$ in both systems. Reprinted with permission from reference 185. Copyright 2007 American Chemical Society.

In addition, there is a clear difference in the behavior of both organozinc derivatives: While $\mathrm{ZnMe}_{2}$ produces trans- $\left[\mathrm{PdR}_{\mathrm{F}} \mathrm{Me}\left(\mathrm{PPh}_{3}\right)_{2}\right]$ at a very high rate that further reacts with $\mathrm{ZnMe}_{2}$ to produce mainly $\mathrm{ZnR} F \mathrm{Me}$ and $\left[\mathrm{PdMe}_{2}\left(\mathrm{PPh}_{3}\right)_{2}\right]$, the use of ZnMeCl produces faster cis- $\left[\mathrm{PdR}_{\mathrm{F}} \mathrm{Me}\left(\mathrm{PPh}_{3}\right)_{2}\right]$ than trans- $\left[\mathrm{PdR}_{\mathrm{F}} \mathrm{Me}\left(\mathrm{PPh}_{3}\right)_{2}\right]$, then the cis isomer isomerizes to give the trans isomer and also reacts with $\mathrm{ZnMeCl}$ leading to $\mathrm{ZnR}_{\mathrm{F}} \mathrm{Cl}$. The isomerization of the palladium complexes in the reaction media is faster than the observed isomerization of the isolated complexes in solution, so it must be catalyzed by $\mathrm{ZnMeCl}$. In fact the role of $\mathrm{ZnMeCl}$ as catalysts for the isomerization trans-cis of complexes $\left[\mathrm{PdMe}_{2} \mathrm{~L}_{2}\right]$ has been demonstrated. ${ }^{186}$

Although the Negishi reaction has proved to be useful in the cross-coupling of perfluoroalkyl groups, no mechanistic studies about the transmetalation of these groups have been reported. 


\subsubsection{Reductive Elimination}

Reductive Elimination from Pd(II) complexes.

The success of a catalytic reaction that transforms fluorinated substrates may rest upon this final step of the cycle. Fluoroalkyls do not participate easily in reductive elimination processes neither from $\mathrm{Pd}(\mathrm{II})$ nor from $\mathrm{Pd}(\mathrm{IV})$ complexes. The reluctance of these groups to participate in this reaction has drastically limited the use of palladium to the catalytic formation of $\mathrm{R}_{\mathrm{F}}-\mathrm{C}$ bonds $\left(\mathrm{R}_{\mathrm{F}}=\right.$ fluoroalkyl). The problem is particularly acute in trifluoromethylation reactions involving $\operatorname{Pd}(0) / \mathrm{Pd}(\mathrm{II})$ systems. Although there are limited reports on the catalytic cross-coupling of perfluoroakyliodides, most of them involve radical reactions or the use of other metals in addition to palladium.

This inertness towards reductive elimination allows the synthesis of $\operatorname{Pd}(\mathrm{II})$ perfluoroalkyl $\left[\mathrm{PdR}_{\mathrm{F}} \mathrm{RL}_{2}\right]$ derivatives by oxidative addition of $\mathrm{R}_{\mathrm{F}} \mathrm{I}$ to dimethyl palladium(II) producing a $\mathrm{Pd}(\mathrm{IV})$ intermediate that eliminates IMe. ${ }^{189,190}$ Culkin and Hartwig prepared several complexes with a aryl and trifluoromethyl groups in relative cis positions such as $\quad\left[\mathrm{Pd}\left(\mathrm{CF}_{3}\right)(p\right.$-tol $\left.)(\mathrm{dppbz})\right] \quad(\mathrm{dppbz}=1,2-$ bis(diphenylphosphino)benzene), and reported its reluctance to produce trifluoromethyltoluene even after being heated at $130{ }^{\circ} \mathrm{C}$ for prolonged periods of time. ${ }^{191}$ The analogous trifluoroethyl complex $\left[\mathrm{Pd}\left(\mathrm{CH}_{2} \mathrm{CF}_{3}\right)(p\right.$-tol $\left.)(\mathrm{dppbz})\right]$ underwent reductive elimination sluggishly to form 1-methyl-4-(2,2,2-trifluoroethyl)benzene in $96 \%$ yield upon heating $36 \mathrm{~h}$ at $110{ }^{\circ} \mathrm{C}$, while the elimination of nonfluorinated alkyl takes place at this temperature in less than one hour.

It is well known than rigid chelating ligands hamper the reductive elimination, thus this reaction has been tested with monodentate ligands in $\left[\mathrm{Pd}\left(\mathrm{CF}_{3}\right)(\mathrm{Ph})\left(\mathrm{PPh}_{3}\right)_{2}\right]$. Although the decomposition of the product takes place at $60^{\circ} \mathrm{C}$ in the presence of $\mathrm{IPh}$, 
the reaction does not produce trifluorotoluene, but instead other decomposition products are formed. ${ }^{192}$ Better results have been obtained by facilitating the reductive elimination employing chelating ligands with wider bite angle than dppbz: ${ }^{193}$ Both $\left[\mathrm{Pd}\left(\mathrm{CF}_{3}\right)(\mathrm{Ph})(\mathrm{dppe})\right]$ and $\left[\mathrm{Pd}\left(\mathrm{CF}_{3}\right)(\mathrm{Ph})(\mathrm{dppp})\right]$ have been reported to yield $10-60 \%$ of $\mathrm{PhCF}_{3}$ but after several hours at $145{ }^{\circ} \mathrm{C},{ }^{163}$ and $\left[\mathrm{Pd}\left(\mathrm{CF}_{3}\right)(\mathrm{Ph})(\mathrm{Xantphos})\right]$ produces the cross-coupling product quantitatively at $80{ }^{\circ} \mathrm{C}$ in only $3 \mathrm{~h} .{ }^{194,192}$ This reaction has been studied in dept by kinetic and computational methods. ${ }^{195}$ It has a small but not negligible dependence on the concentration of Xantphos in the reaction medium, and the kinetics show a saturation effect on [Xantphos], being independent of it when more than two equivalents of Xantphos are added to the $c i s-\left[\mathrm{Pd}\left(\mathrm{CF}_{3}\right) \mathrm{Ph}(\mathrm{Xantphos})\right]$ solution. The activation parameters were obtained in absence of extra Xantphos $\left(\Delta H^{*}=25.9 \pm\right.$ $2.6 \mathrm{kcal} \mathrm{mol}^{-1}$ and $\Delta \mathrm{S}^{*}=6.4 \pm 7.8$ e.u.) and they were also measured in the presence of 2 equiv of Xantphos: $\Delta \mathrm{H}^{\ddagger}=29.3 \pm 2.8 \mathrm{kcal} \mathrm{mol}^{-1}$ and $\Delta \mathrm{S}^{\ddagger}=15.9 \pm 8.4$ e.u. From the kinetic analysis of the reaction and with the additional help of calculated energies for the different possible mechanisms, the authors propose that at least two competitive pathways are operating (Scheme 58). In one of them the reductive elimination takes place in the square-planar complex cis- $\left[\mathrm{Pd}\left(\mathrm{CF}_{3}\right) \mathrm{Ph}(\mathrm{Xantphos})\right]$ while in the presence of an excess of phosphine the reaction proceeds mainly through cis$\left[\mathrm{Pd}\left(\mathrm{CF}_{3}\right) \mathrm{Ph}(\text { Xantphos })_{2}\right]$.

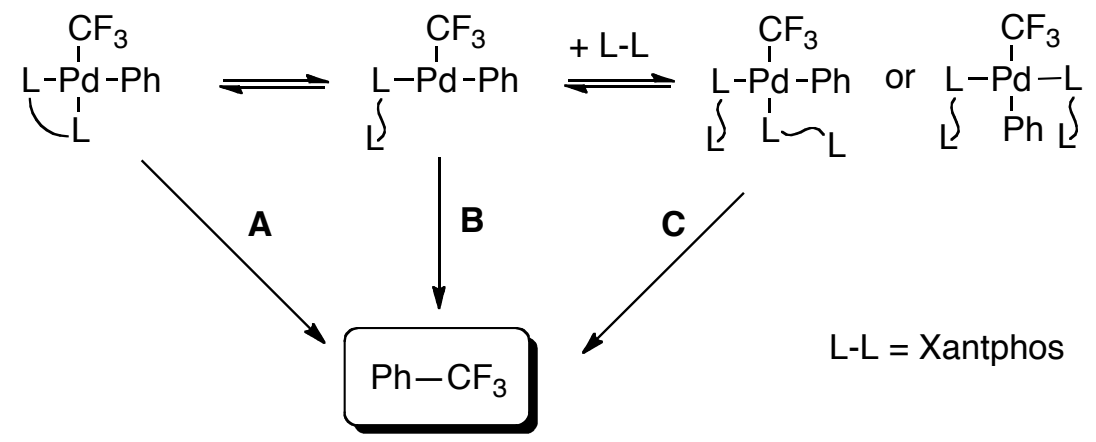

Scheme 58 
The calculated energies of activation for the reductive elimination from $\mathbf{A}$ or $\mathbf{C}$ (Scheme 58) have values about 25 and $30 \mathrm{kcal} \mathrm{mol}^{-1}$ in good agreement with the experimental values, while the activation energies starting from possible intermediates in which one phosphorous atom of the phosphine is not coordinated reach much higher values, around $40 \mathrm{kcal} . \mathrm{mol}^{-1}$. Also, very high activation energies are obtained for other coordination modes of the phosphine, such as coordination of the oxygen or of one aryl group.

Buchwald's group succeeded in performing a catalytic cycle by using BrettPhos or RuPhos as supporting ligands in which the reductive elimination is the slowest step. ${ }^{60}$ Kinetic experiments carried out on the isolated intermediates $\left[\mathrm{PdArCF}_{3}(\mathrm{BrettPhos})\right]$ showed that the activation energy for the reductive elimination step is almost independent on the aryl group. This behavior has been explained with the aid of computational studies that show that the energy of the transition state for this step is mainly due to the elongation of the $\mathrm{Pd}-\mathrm{CF}_{3}$ bond, while the $\mathrm{Pd}-\mathrm{Ar}$ bond has little influence on it. This fact, along with high strength of the $\mathrm{M}-\mathrm{CF}_{3}$ bond discussed above, explain the kinetic inertness of this group to produce reductive elimination reactions from palladium(II) complexes. However, the role of the different supporting ligands remains unclear and no satisfactory explanation for the reasons why some of them promote the reactions much better than others has been provided.

This issue has been addressed by Anstaet and Schoenebeck, who computationally studied the differences in the reductive elimination of the model complexes $\left[\mathrm{Pd}\left(\mathrm{CF}_{3}\right) \mathrm{Ph}(\mathrm{dppe})\right]$ and $\left[\mathrm{Pd}\left(\mathrm{CF}_{3}\right) \mathrm{Ph}(\mathrm{Xantphos})\right] .{ }^{196}$ The calculated energies give values about $8 \mathrm{Kcal} \mathrm{mol}^{-1}$ higher for the activation energy of the complex with dppe than with Xantphos. The authors correlate this effect with the different bite angle 
of both ligands, but mainly with the different steric effects in the transition and ground states: While for dppe the steric interactions increase on going from the ground state to the TS, the opposite rules for the Xantphos. The phenyl groups as substituents at the phosphorus in both ligands play a fundamental role in these steric interactions, and the trends change when other substituents are calculated.

The strength of the $\mathrm{M}-\mathrm{Ar}_{\mathrm{F}}$ bonds and thus the stability of the $\left[\operatorname{PdRAr}_{\mathrm{F}} \mathrm{L}_{2}\right]$ intermediates also lead to high energy barriers for the reductive elimination step in the case of fluoroaryl derivatives, although this reaction is not as sluggish as the one for trifluromethyl derivatives. This process was analyzed by DFT calculations by Peruzt, Eisenstein et al. on phenyl fluoroaryl derivatives and they found the largest activation energies for ortho fluorinated aryls as discussed in section 2.2.2. ${ }^{168}$

The efficiency of the Stille coupling of allylic halides and aryltin derivatives is controlled by the reductive elimination, which is the slow step for this particular combination of reactants. This was found and studied in detail using a fluoroaryl derivative $\left(\mathrm{C}_{6} \mathrm{~F}_{3} \mathrm{Cl}_{2}\right)$ and a fluoroaryl-labeled allylic fragment. $\mathrm{A} \eta^{3}$-allyl ( $\mu$-fluoroaryl) dimeric complex was independently prepared (Scheme 59). ${ }^{109}$ Even this "ligandless" complex is reluctant to reductive elimination and, instead, decomposes upon heating by $\beta-\mathrm{H}$ elimination, and the products derived from the $\mathrm{C}_{6} \mathrm{~F}_{3} \mathrm{Cl}$ and $\mathrm{C}_{6} \mathrm{~F}_{5}$-allyl groups are conveniently recognized and analyzed by ${ }^{19} \mathrm{~F}$ NMR. The reductive elimination has to be promoted by benzoquinone, an electron withdrawing ligand, which coordinated to give a mononuclear complex that undergoes a clean reductive elimination. ${ }^{197}$ Benzoquinone was then used as coupling co-catalyst and produced a more efficient Stille reaction of fluoroarylorganotin derivatives and allylic halides, circumventing the coupling problems. 


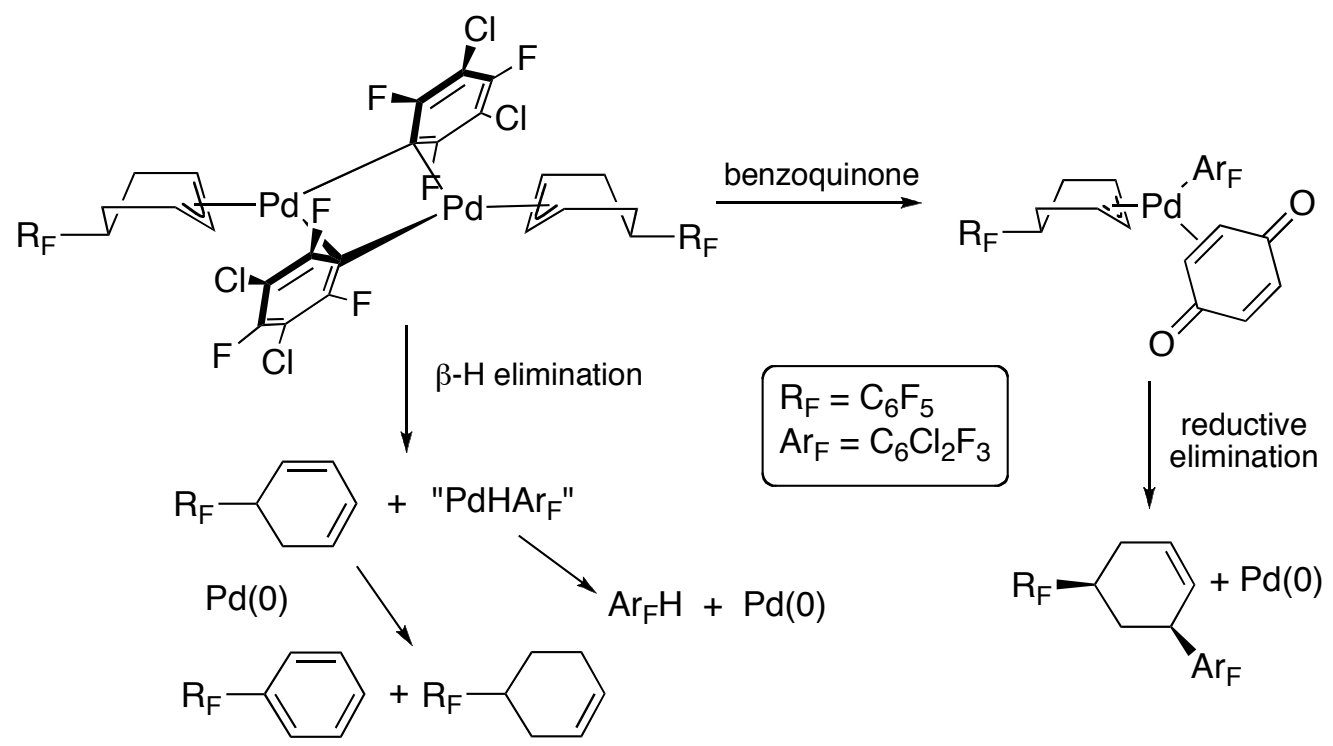

Scheme 59

The decrease of the electron density of the metal is a factor that facilitates the reductive elimination and, in an extreme, this can be effected by oxidation. The addition of strong oxidants may form $\mathrm{Pd}(\mathrm{III})$ or $\mathrm{Pd}(\mathrm{IV})$ intermediates that are becoming more relevant specially in fluoroalkyl couplings and fluorination reactions (see below). Some reductive eliminations promoted by oxidants have been reported. The oxidation of $\operatorname{Pd}(\mathrm{II})$ to induce the reductive elimination of perfluoroaryl groups has been used by Fornies' group to produce C-P bonds (Scheme 60). ${ }^{198,199}$ The reaction with $\mathrm{I}_{2}$ presumably occurs with addition of one iodide on one of the Pd atoms of the dimer, inducing the $\mathrm{PR}_{2}-\mathrm{Ar}_{\mathrm{F}}$ reductive elimination on the other one with subsequent rearrangement to form a bridging iodine.

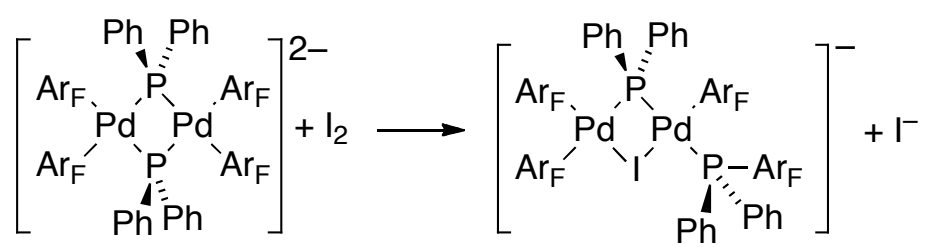

Scheme 60 
Reductive elimination from $\mathrm{Pd}(\mathrm{IV})$ complexes.

Contrary to the behavior of $\mathrm{Pd}(\mathrm{II})$, the reductive elimination of $\mathrm{C}\left(\mathrm{sp}^{3}\right)$ in $\mathrm{Pd}(\mathrm{IV})$ complexes is faster than the elimination of $\mathrm{C}\left(\mathrm{sp}^{2}\right)$, leading to the alkyl fluorinated products with very high selectivity.

The first demonstration of $\mathrm{Ar}-\mathrm{CF}_{3}$ bond-forming reductive elimination from a $\mathrm{Pd}^{\mathrm{IV}}$ aryl trifluoromethyl complex was provided by Sanford's group in $2010{ }^{200}$ The synthesis of $\left[\mathrm{PdAr}\left(\mathrm{CF}_{3}\right)(\mathrm{LL})\right](\mathrm{LL}=\mathrm{N}, \mathrm{N}$ or $\mathrm{P}, \mathrm{P}$ chelating ligands) was accomplished by reaction of the $\mathrm{Pd}^{\mathrm{II}}$ aryl iodides $[\mathrm{PdArI}(\mathrm{LL})]$ with $\mathrm{CsF}$ and $\mathrm{Me}_{3} \mathrm{SiCF}_{3}$. The oxidation of these complexes with $N$-fluoro-2,4,6-trimethylpyridinium triflate (NFTPT) as oxidant render a trifluoromethyl palladium(IV) complex with the ligand 4,4'ter-butyl2,2'bipyridine (dtbpy) (Scheme 61).
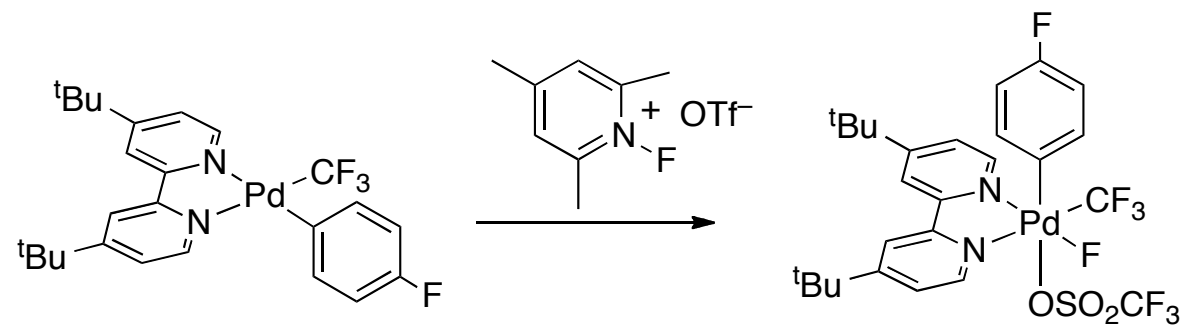

Scheme 61

When performed at $80^{\circ} \mathrm{C}$, the oxidation is immediately followed by a reductive elimination process to give the cross-coupling products $4-\mathrm{FC}_{6} \mathrm{H}_{4}-\mathrm{CF}_{3}$. Thus, the reductive elimination from $\mathrm{Pd}(\mathrm{IV})$ seems to be kinetically much easier than from $\mathrm{Pd}(\mathrm{II})$. Remarkably, the reaction does not produce other reductive elimination products such as $\mathrm{Ar}-\mathrm{F}$.

The reaction mechanism has been studied experimentally and by DFT computational analysis. ${ }^{201}$ First they demonstrated that, as expected, the reductive elimination of $\mathrm{Ar}-\mathrm{CF}_{3}$ does not take place from the $\mathrm{Pd}(\mathrm{II})$ complex $\left[\mathrm{PdAr}\left(\mathrm{CF}_{3}\right)(\mathrm{LL})\right]$. 
Heating the complex $\left[\mathrm{Pd}\left(\mathrm{CF}_{3}\right)\left(4-\mathrm{F}-\mathrm{C}_{6} \mathrm{H}_{4}\right)(\mathrm{dtbpy})\right]$ at $130{ }^{\circ} \mathrm{C}$ for $72 \mathrm{~h}$ only a $5 \%$ of the cross coupling product $\mathrm{CF}_{3}-\mathrm{C}_{6} \mathrm{H}_{4} \mathrm{~F}$ was obtained. The reagent used for the oxidation to $\mathrm{Pd}(\mathrm{IV})$ plays a fundamental role in the reaction, since it provides different molecular frames that can produce competing reductive elimination reactions, and in fact they do (Scheme 62). For instance, the use of N-bromo-succinimide produces the elimination of $\mathrm{Ar}-\mathrm{Br}$ as major product. Only electrophilic fluorinating reagents give the desired coupling product, due to the difficulty on the elimination of fluoride from Pd(IV).

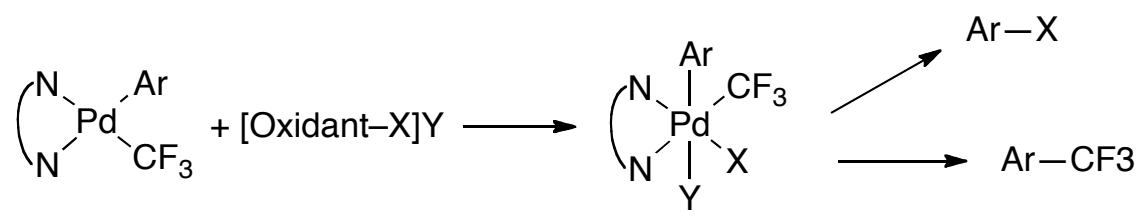

Scheme 62

The kinetics of the decomposition reaction of the complex $\left[\mathrm{PdF}\left(\mathrm{CF}_{3}\right)(4-\mathrm{F}-\right.$ $\left.\left.\mathrm{C}_{6} \mathrm{H}_{4}\right)(\mathrm{OTf})(\mathrm{dtbpy})\right]$ shows a reverse dependence on the concentration of triflate, suggesting a pre-dissociation equilibrium of this anion; in fact the replacement of this anion by $\mathrm{PF}_{6}^{-}$produces a significant increase in the decomposition rate. The activation parameters of this reaction, $\Delta \mathrm{H}^{\ddagger} 29.1(0.2) \mathrm{kcal} \mathrm{mol}^{-1}$ and $\Delta \mathrm{S}^{\ddagger} 9.48(0.8) \mathrm{cal} \mathrm{mol}^{-1} \mathrm{~K}^{-1}$ support a mechanism in which one ligand have left the coordination sphere during or previously to the rate determining step.

Electronic effects on reductive elimination were evaluated, resulting in that electron-donating aryl substituents accelerate the reaction. This may be interpreted as a strongest trans-labilization effect resulting in an easier dissociation of the trans-triflate ligand, but also the stabilization of the pentacoordinate intermediate or of the transition state may have a significant contribution to the enhanced reaction rate (Scheme 63). The analysis by DFT calculations of the atomic charges of the Pd(IV) complex indicates that the trifluoromethyl carbon carries a significant positive charge $(+1.18)$, larger than in 
Pd(II) complexes, ${ }^{164}$ wheras the ipso-carbon of the phenyl ligand bears a charge of +0.07 . Based on this difference in the atomic charges the authors propose that the aryl group acts as nucleophile towards the trifluoromethyl during the reaction pathway, what additionally explain the electronic effect on the aryl groups.

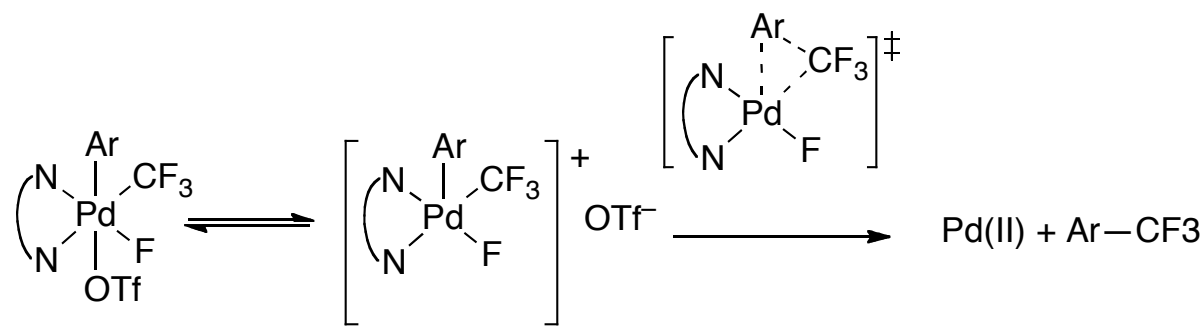

Scheme 63

\subsubsection{2,1-Insertion}

Since the $\mathrm{Pd}-\mathrm{Ar}_{\mathrm{F}}$ bond of polyfluorinated aryls is stronger that an analogous non-fluorinated Pd-Ar bond, the activation energy for 2,1-insertion of an alkene into a $\mathrm{Pd}-\mathrm{Ar}_{\mathrm{F}}$ bond is expected to be higher. This being true, the reaction is possible and many stoichiometric examples have been reported as well as catalytic examples were this step is involved in the catalytic cycle, such as the Heck coupling. ${ }^{86,87}$

${ }^{19}$ F NMR spectroscopy offers a very convenient tool to study these reaction since the chemical shift difference of the ortho fluorine in polyfluorinated aryls change about 20 ppm upfield on going from a $\mathrm{Pd}-\mathrm{Ar}_{\mathrm{F}}$ to a $\mathrm{C}-\mathrm{Ar}_{\mathrm{F}}$ linkage. ${ }^{202}$ In this way we have studied many stoichiometric examples of insertion of an alkene into the $\mathrm{Pd}-\mathrm{C}_{6} \mathrm{~F}_{5}$ bond.

As in any 2,1-insertion of alkenes into a Pd-R bond, coordination of the olefin and a cis in-plane arrangement is needed for insertion. Thus the complex $\left[\operatorname{Pd}\left(\mathrm{C}_{6} \mathrm{~F}_{5}\right) \mathrm{Br}(\right.$ bipy $\left.)\right]$ does not react with alkenes, but insertion takes place immediately when the blocking $\mathrm{Br}$ ligand is removed with $\mathrm{AgClO}_{4}$. When dienes are used $\eta^{3}$-allyl palladium derivatives are formed which can be isolated and characterized. Under the 
same conditions and reaction times, trans- $\left[\mathrm{Pd}\left(\mathrm{C}_{6} \mathrm{~F}_{5}\right) \mathrm{BrL}_{2}\right]\left(\mathrm{L}=\right.$ py, $\left.\mathrm{PPh}_{3}\right)$ and $\mathrm{AgClO}_{4}$ gives only trans- $\left[\mathrm{Pd}\left(\mathrm{C}_{6} \mathrm{~F}_{5}\right)\left(\mathrm{OClO}_{3}\right) \mathrm{L}_{2}\right]($ Scheme 64$) .{ }^{203}$

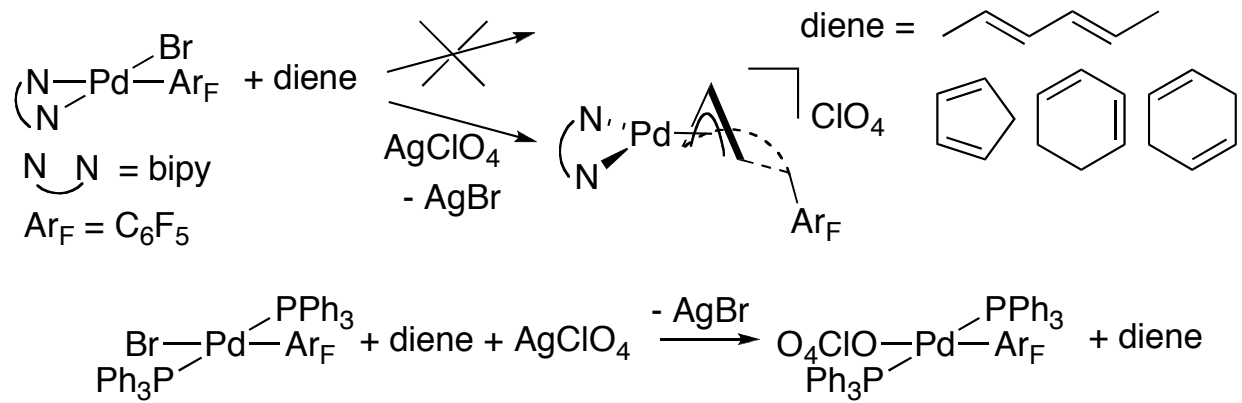

Scheme 64

$\left[\operatorname{PdBr}\left(\mathrm{C}_{6} \mathrm{~F}_{5}\right)(\mathrm{COD})\right](\mathrm{COD}=1,5$-cyclooctadiene $)$ is a stable cis-aryl-olefin complex due to the impossibility of the double bond cis to the aryl group to become coplanar with the $\mathrm{Pd}-\mathrm{Ar}_{\mathrm{F}}$ bond unless the other double bond in the chelating alkene decoordinates. Its X-ray structure shows a longer Pd-double bond length trans to $\mathrm{C}_{6} \mathrm{~F}_{5}$, reflecting the high trans influence of the pentafluorophenyl group and supporting a preferred decoordination of that trans double bond. This decoordination is rate determining for insertion, and when it occurs (smoothly at room temperature) the compound evolves fast and competitively to the kinetic, $\sigma, \eta^{2}$-cyclooctenyl- $(\mathbf{A})$ and the thermodynamic $\eta^{3}$-cyclooctenyl (B) palladium products, via the putative $\sigma$-cyclooctenyl intermediate I shown in Scheme 65. Both final products have a cis stereochemistry for the $\operatorname{Ar}_{\mathrm{F}}$ and $\mathrm{Pd}$ groups (determined by NOE for $\mathbf{B}$ and by X-ray diffraction of a derivative for A) supporting the expected cis addition. The competitive formation of both products prove the involvement of a common intermediate $\mathbf{I}$; the $\sigma, \eta^{2}$-cyclooctenyl (A) complex is not an intermediate, since its transformation into the allylic $\eta^{3}$ cyclooctenyl (B) is extremely slow (again a rate determining decoordination of the double bond is needed). The $\mathrm{Pd}$ migration (or chain walking by $\beta-\mathrm{H}$ elimination- 
readdition) to give $\mathbf{B}$ and the recoordination of the double bond to give $\mathbf{A}$ from the common intermediate have comparable rates, and are fast. ${ }^{204}$

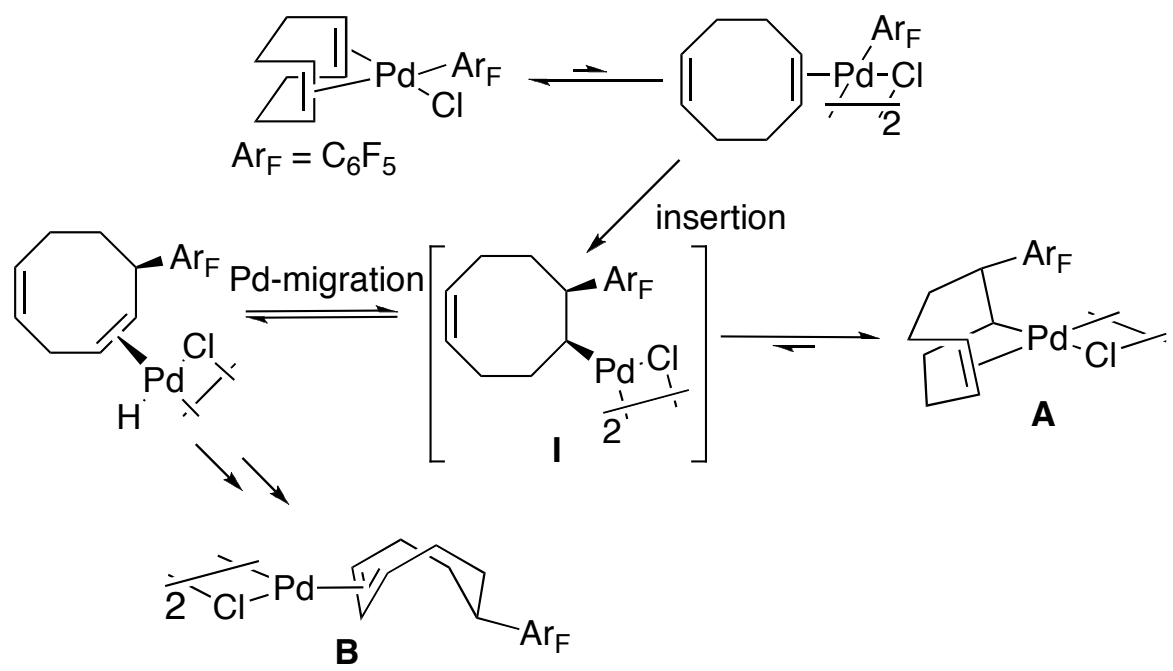

Scheme 65

Thus, insertion of the double bond into the $\mathrm{Pd}-\mathrm{C}_{6} \mathrm{~F}_{5}$ moiety is fast whenever a non-chelating alkene and a palladium fluoroaryl complex with labile ligands, such as $\left[\operatorname{PdBr}\left(\mathrm{C}_{6} \mathrm{~F}_{5}\right)(\mathrm{NCMe})_{2}\right]$, are used. No monodentate alkene complex could be detected even at low temperature for these reactions showing that when coordination of the olefin occurs and a cis coplanar arrangement can be reached, the insertion of the alkene into the $\mathrm{Pd}-\mathrm{C}_{6} \mathrm{~F}_{5}$ bond is feasible and fast. Many pentafluorophenyl allyl palladium complexes have been synthesized by the insertion reaction of a double bond of a diene into the $\mathrm{Pd}-\mathrm{C}_{6} \mathrm{~F}_{5}$ bond of $\left[\mathrm{PdBr}\left(\mathrm{C}_{6} \mathrm{~F}_{5}\right)(\mathrm{NCMe})_{2}\right] .{ }^{202}$ The chemo and regiochemistry of the insertion can be determined by the analysis of the structure of the final compound. Insertion of the less substituted bond occurs and the $\mathrm{C}_{6} \mathrm{~F}_{5}$ group always binds the least substituted position of the alkene (i.e. 2,1-insertion, Scheme 66). ${ }^{205}$ 
ratio diene

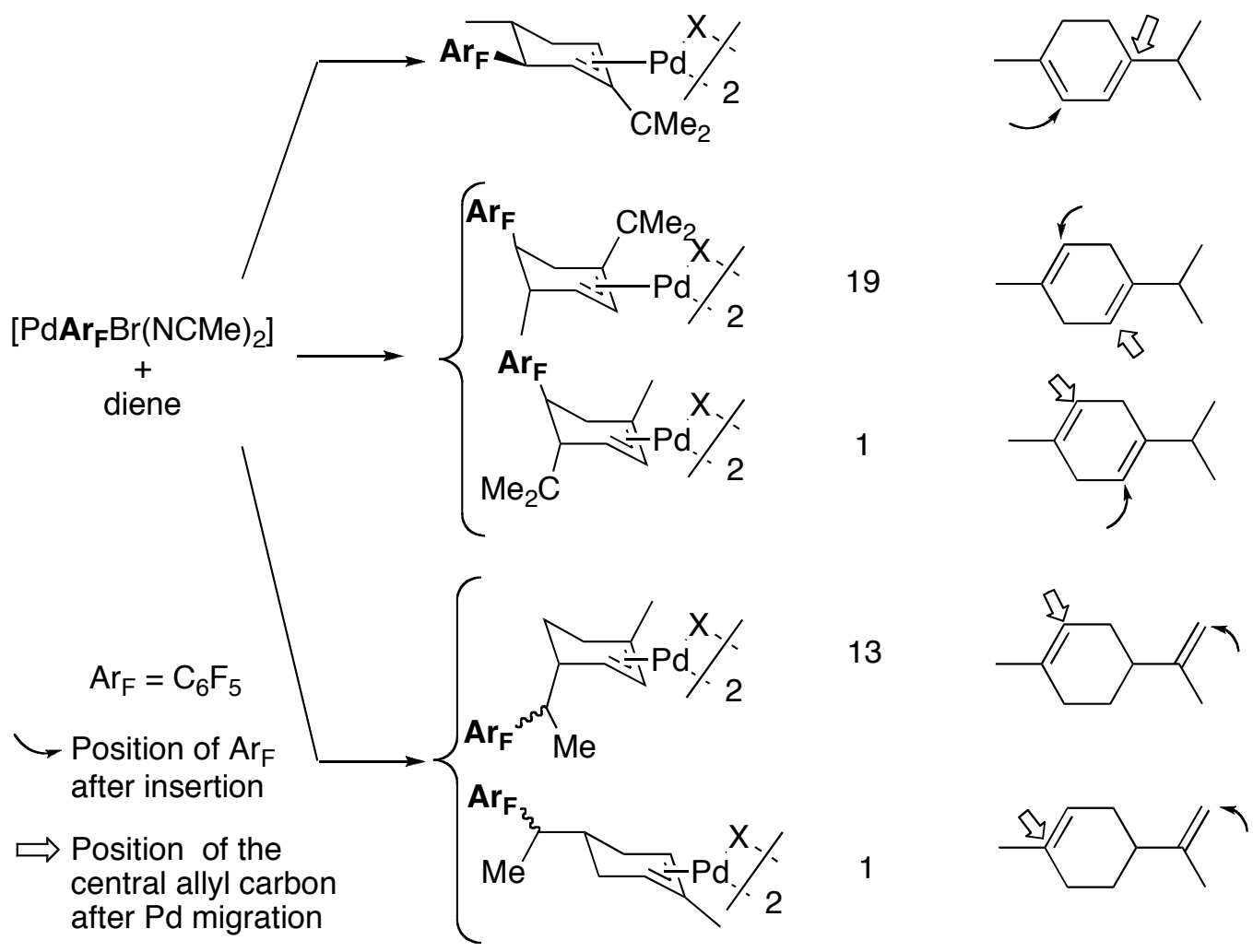

Scheme 66

The presence of a fluorinated group and the use of ${ }^{19} \mathrm{~F}$ NMR aids the characterization of product mixtures and allows the monitorization of processes that occur on the organometallic fragment after insertion of the alkene into the $\mathrm{Pd}-\mathrm{C}_{6} \mathrm{~F}_{5}$ bond. Thus, palladium migration along a hydrocarbon chain (chain walking) has been studied in detail and relevant intermediates have been detected, as snapshots of the reaction course. ${ }^{205-207}$ The fate of the palladium hydride after $\beta-\mathrm{H}$ elimination and its involvement in secondary reduction processes of relevance in catalysis have also been ascertain using $\mathrm{C}_{6} \mathrm{~F}_{5}$ labeled organometallic fragments. ${ }^{208-211}$ Other $\beta$-elimination processes have also been studied such as $\beta$-OR, $\beta$-OC(O), $\beta-\mathrm{Cl}, \beta-\mathrm{SiR}_{3}$, and $\beta$-SR eliminations. $^{212,213}$ Intramolecular alkene insertions and skeletal C-C rearrangements have also been analyzed with the aid of the fluorinated group as a spectroscopic label. ${ }^{214}$ 
Alkene insertion into a $\mathrm{Pd}-\mathrm{Ar}_{\mathrm{F}}$ bond is a fundamental step in the Heck couplings shown in Scheme 67 along with the mechanism of the reaction. ${ }^{86,87}$ The dimeric complex $\left(\mathrm{NBu}_{4}\right)_{2}\left[\mathrm{Pd}(\mu-\mathrm{Br}) \mathrm{Br}\left(\mathrm{C}_{6} \mathrm{~F}_{5}\right)\right]_{2}$ is used as catalyst precursor and it is in equilibrium with the halo or halo-solvated palladium complexes shown. Coordination of the alkene to these species is followed by insertion, but these two different elementary steps could not be experimentally studied in a separate way. We observed that either the oxidative addition or the coordination-insertion steps can be rate limiting depending on the actual $\operatorname{Ar}_{\mathrm{F}}$ used and the reaction conditions. When a penta or tetrafluroaryl bromide are used the coordination-insertion is rate limiting in the catalysis. However, if a large excess of the alkene is added, in order to increase the rate of coordination-insertion, the coordination of the alkene to $\operatorname{Pd}(0)$ species in the cycle makes them more stable and the oxidative addition step slower. Thus, this action changes the rate determining step of the reaction to oxidative addition for $p-\mathrm{RC}_{6} \mathrm{~F}_{4} \mathrm{Br}$ when $\mathrm{R}=\mathrm{OMe}, \mathrm{F}$ producing the opposite to the expected effect. If $\mathrm{R}$ is an electron withdrawing group such as $\mathrm{CF}_{3}$ or $\mathrm{CN}$ the oxidative addition is still fast and the coordination-insertion step remains the slowest one in the cycle even in the presence of an excess of olefin. The monofluorinated $p$ fluorophenyl bromide behaves differently and the oxidative addition step, expected to be slower, is rate limiting in any reaction condition. This results show that insertion of the alkene into the stronger $\mathrm{Pd}-\mathrm{Ar}_{\mathrm{F}}$ of polyfluorinated aryls is less favored when compared to non-fluorinated aryls indeed and may be rate limiting. 


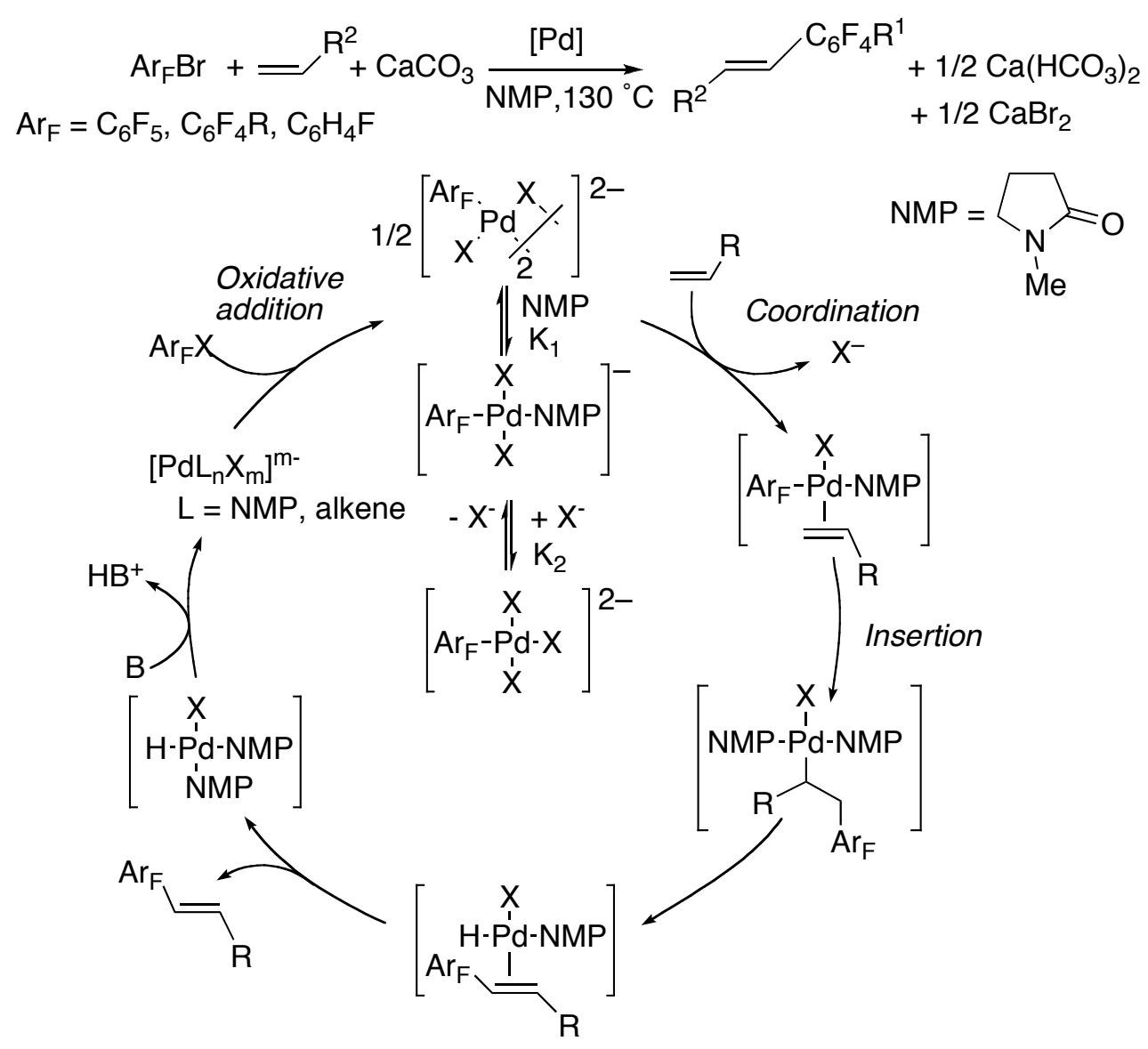

Scheme 67

When reacting with palladium complexes, alkenyl organometallic derivatives are susceptible of a competition between transmetalation and alkene insertion and this can affect the regiochemistry and selectivity of the catalytic reaction. This competition was studied using substituted alkenyl silanes in such a way that the products derived from insertion (Heck type products and $\beta-\mathrm{SiR}_{3}$ elimination routes) would be different from those expected from transmetalation and reductive elimination (Hiyama). Palladium pentafluorophenyl complexes were used, so the advantageous spectroscopic features of the $\mathrm{C}_{6} \mathrm{~F}_{5}$ group would aid in the characterization of the final products. Scheme 68 shows the products obtained for 2-propenyl trimethyl silane. Other alkenyl silanes and ligands were also tried but, in every case, insertion of the olefin into the Pd$\mathrm{C}_{6} \mathrm{~F}_{5}$ bond is the major pathway observed. ${ }^{215}$ 


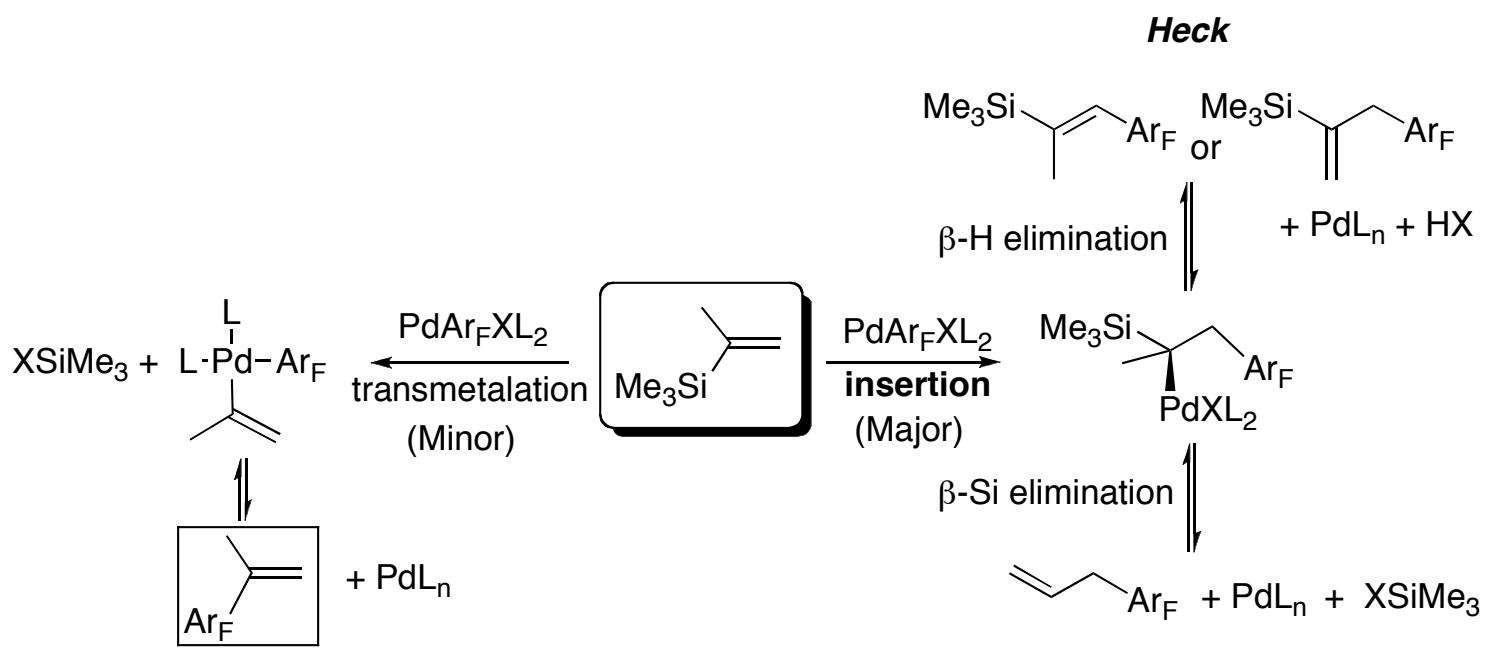

Hiyama

Scheme 68

\subsubsection{1,1-Insertion (migratory insertion)}

Examples of a formal 1,1-insertion of a ligand into a $\mathrm{Pd}-\mathrm{Ar}_{\mathrm{F}}$ bond are known for isonitriles and carbenes. The most common carbon monoxide insertion to give the acyl compound, $\mathrm{Pd}-\mathrm{CO}-\mathrm{Ar}_{\mathrm{F}}$, is not favored, and the equilibrium is shifted towards free $\mathrm{CO}$ and $\mathrm{Pd}-\mathrm{Ar}_{\mathrm{F}}$. Thus, perfluorofluoroaryl acyl halides have been used as precursors of $\operatorname{Ar}_{\mathrm{F}}$ and one example is shown in Scheme $69 .{ }^{216}$ Complete decarbonylation of the acyl group is observed for $\operatorname{Ar}_{\mathrm{F}}=\mathrm{C}_{6} \mathrm{~F}_{5}$ but the selectivity decreases for other less fluorinated aryls. For example, when $\mathrm{Ar}_{\mathrm{F}}=3,5-\mathrm{F}_{2} \mathrm{C}_{6} \mathrm{H}_{3}$ decarbonylation is a minor pathway and the product of the reaction is a mixture of the aryl trimethylsilane (minor) and the acyl trimethylsilane (major).

$$
\mathrm{C}_{6} \mathrm{~F}_{5}-\mathrm{Cl}_{\mathrm{Cl}}^{\mathrm{O}}+\mathrm{Si}_{2} \mathrm{Me}_{6} \underset{-\mathrm{CO}}{\stackrel{[\mathrm{Pd}]}{\longrightarrow}} \mathrm{C}_{6} \mathrm{~F}_{5}-\mathrm{SiMe}_{3}+\mathrm{ClSiMe}_{3}
$$

Scheme 69

The insertion of isonitriles into $\mathrm{Pd}-\mathrm{Ar}_{\mathrm{F}}$ bonds occurs, upon heating, on $\mathrm{Pd}$ complexes containing both groups $\left(\mathrm{Ar}_{\mathrm{F}}\right.$ and $\left.\mathrm{CNR}\right)$ coordinated to the metal to give 
Pd(II) imidoyl complexes (Scheme 70). ${ }^{217,218}$ The initial product is a crown-shaped tetrameric complex. Controlled addition of $\mathrm{L}$ affords boat shaped dimers with the $\operatorname{Ar}_{\mathrm{F}}$ and $\mathrm{R}$ substituents cis relative to the $\mathrm{C}=\mathrm{N}$ bond in the bridging imidoyl ligands.

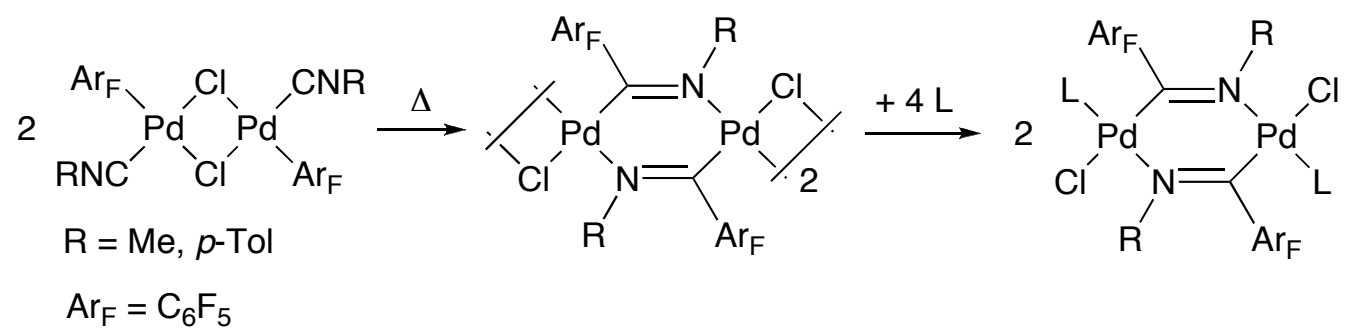

Scheme 70

1,1-insertion of carbenes into Pd-R bonds is a reaction involved in many catalytic processes developed in the last years. ${ }^{219}$ The reaction was disclosed not long ago, by studying the evolution of pentafluorophenyl palladium carbenes. ${ }^{220,221}$ Scheme 71shows two of these reactions where the resulting palladium alkyl complex is stabilized, in the presence of an additional double bond, by formation of a palladium $\eta^{3}$ allyl or $\eta^{3}$-benzyl complex $(X=\mathrm{OMe})$ or an iminium salt $\left(\mathrm{X}=\mathrm{NEt}_{2}\right){ }^{222}$ The reaction is intramolecular, as studied for the isolated monamino palladium carbenes, and faster the more electrophilic the carbene ligand in the order: $\mathrm{X}, \mathrm{R}=\mathrm{NR}_{2}{ }_{2}, \mathrm{NR}_{2}{ }_{2}<<\mathrm{R}, \mathrm{NR}_{2}{ }_{2}<\mathrm{R}$, OR'.

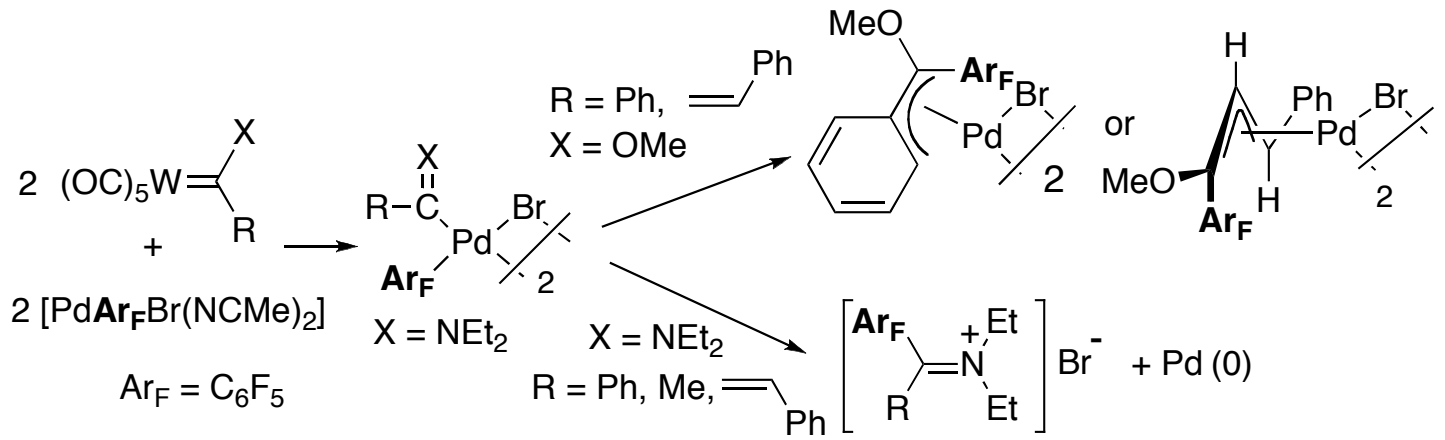

Scheme 71 
The carbene complexes in Scheme 71 were synthesized by transmetalation of a carbene fragment from tungsten to palladium. However other carbene precursors, such as diazoderivatives, have been proposed to give palladium carbene complexes quite reactive towards 1,1-insertion. Trifluoromethyl substituted diazo compounds have been used in the synthesis of $\mathrm{CF}_{3}$ substituted alkenes or dienes. It has been proposed that after oxidative addition of the organic bromide and carbene transfer, 1,1-insertion of the carbene into the Pd-R bond occurs (Scheme 72). ${ }^{223}$

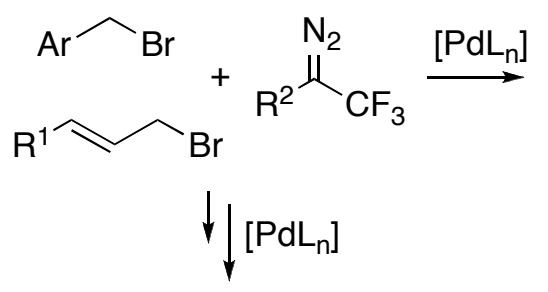<smiles>[R7]C(C=CC=C([R])C(F)(F)F)=C[Bi]</smiles>

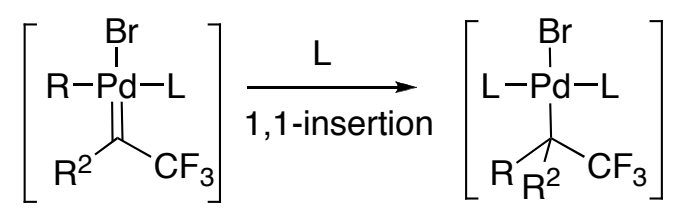

Scheme 72

\section{C-F activation and fluorination.}

Palladium mediated reactions that involve the cleavage or the formation of a C-F bond are included in this section. These reactions are specially challenging from a fundamental point of view since the C-F bond is a strong one and generally difficult to activate. On the other hand, the selective introduction of fluorine in a molecule without the use of aggressive and difficult to handle sources of a fluoro group such as fluorine or hydrogen fluoride, is an attractive goal. In fact, the high reactivity of these fluorine sources generally leads to perfluorinated derivatives. So, the selective cleavage of one 
or several $\mathrm{C}-\mathrm{F}$ bonds in those molecules followed by a suitable functionalization is a way to obtain partially fluorinated compounds of high interest. Reviews with an extensive coverage of the subjects of C-F activation, ${ }^{6,7,224-226}$ and fluorination ${ }^{10,227,228}$ are available in the literature. The purpose of this section is to cover palladium mediated processes in the area. Overviews of the reported palladium catalyzed processes that functionalize a C-F bond on one hand or create a new C-F bond on the other are given below. The current understanding of the fundamental processes that make these reactions possible will follow.

\subsection{Overview of catalytic $\mathrm{C}-\mathrm{C}$ and $\mathrm{C}-\mathrm{X}$ coupling reactions where a $\mathrm{C}-\mathrm{F}$}

\section{bond is cleaved.}

\subsubsection{C-C Coupling reactions of fluorinated aryls.}

Coupling reactions on fluorinated aryl derivatives that involve a C-F cleavage and $\mathrm{C}-\mathrm{C}$ bond formation have been reported using the Stille, Suzuki or Kumada reactions.

The Suzuki reaction has been used to functionalize a C-F bond and leads to an aryl for fluorine substitution. Both monofluorinated and poly- or perfluorinated aryls have been used as reagents and efficient reactions have been developed only for aryl groups that bear additional electron withdrawing substituents. The first examples were reported by Widdowson et at. who carried out the Suzuki reaction on chromium fluoroarene complexes (Scheme 73). ${ }^{229}$ Monofluorinated nitro arenes have also been used, ${ }^{230-232}$ and the presence of an ortho nitro group is essential for the reaction, the C-F bond remaining untouched if the ortho-nitro group is lacking (Scheme 74). ${ }^{231}$ The authors suggest that the ortho nitro group is playing a role that surpass the mere electronic influence since if the $o-\mathrm{NO}_{2}$ is replaced by a trifluoromethyl group the 
reaction does not proceed. They propose that the nitro group can coordinate to palladium facilitating the oxidative addition step (see section 3.4). The presence of additional electron withdrawing groups $\left(\mathrm{R}^{1}\right.$, Scheme 74$)$ is also needed to obtain good or moderate yields. $^{230,231}$

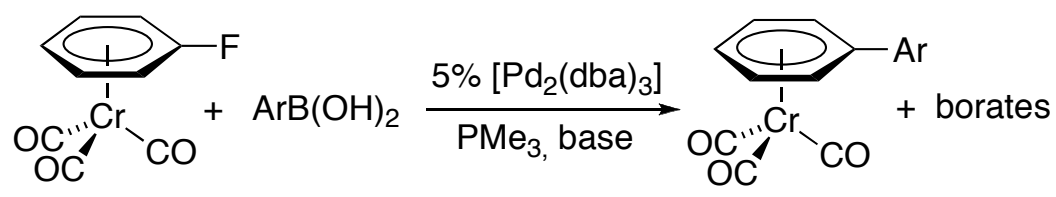

Scheme 73

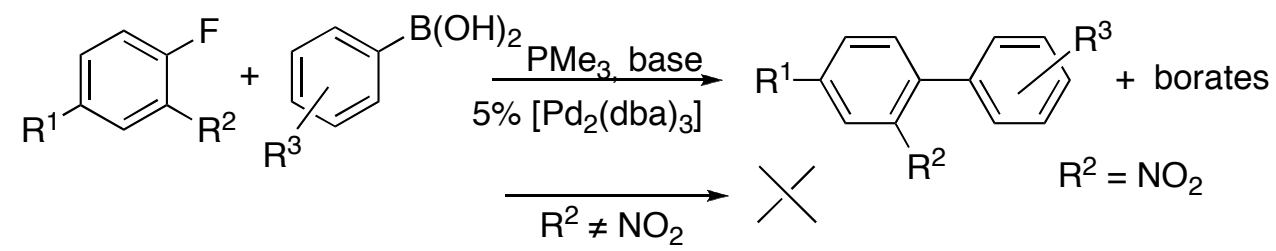

Scheme 74

The Stille coupling has also been carried out with moderate yields using the ortho-fluoro nitroarenes represented in Scheme 74 and aryltin reagents. As a result, substitution of the ortho fluorine occurs and diarenes are formed. ${ }^{230}$

When polyfluorinated nitroarenes are tested in Suzuki reactions the C-F bond ortho to the nitro group in the aromatic ring is selectively cleaved and the reactivity increases with the number of fluoro substituents. Thus pentafluoronitrobenzene is the most reactive fluoroarene followed by tetra- and trifluoronitrobenzene. ${ }^{233}$ This is consistent with the need of an arene as electrophilic as possible. Also, the reactivity observed for different isomeric tetra- and trifluoronitrobenzenes correlates with the activating ability of the ortho, meta and para substituents towards an $\mathrm{S}_{\mathrm{N}} \mathrm{Ar}$ substitution indicating that, regardless the intimate mechanism of the C-F activation, an interaction of the nucleophilic the palladium complex with an electrophilic arene is taking place (Scheme 75). 

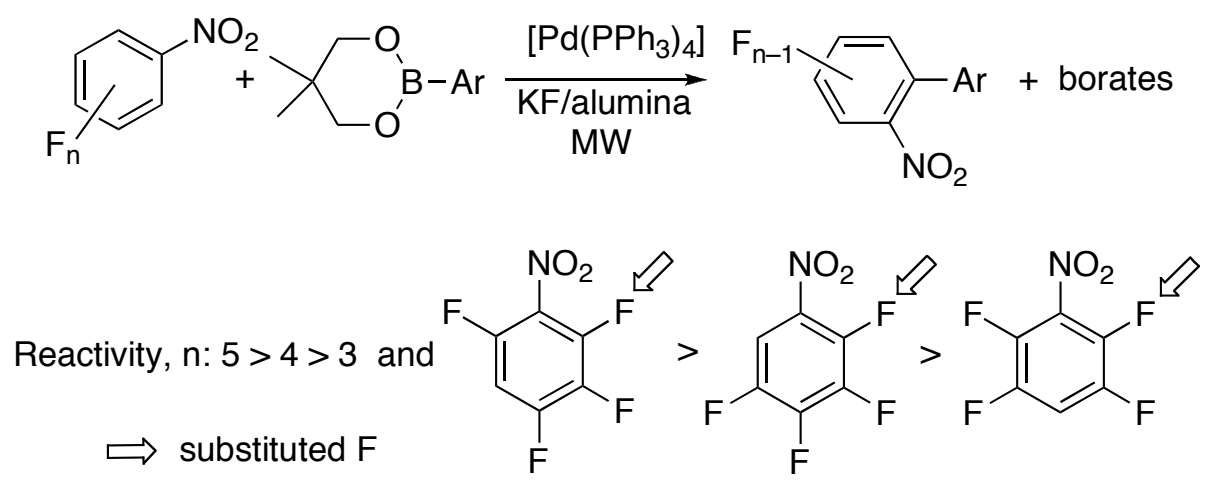

Scheme 75

C-F functionalization by the Suzuki and also by the Stille reaction was observed for 2-fluoro carboxylic acids. Although the yields are lower than those obtained for the analogous nitro derivatives, the reactivity trends observed are the same: The ortho carboxylic group is essential as well as the presence of additional electron withdrawing groups. ${ }^{234}$ The Suzuki coupling has been carried out successfully for pentafluorophenyl oxazolines with a large variety of boronic acids, although the reaction is ineffective for the monofluoro analogues. In this case the same trends as those mentioned for the nitro derivatives are observed: Selective functionalization of the ortho fluorine and lower reactivity as the number of fluorine atoms in the ring decrease. ${ }^{235}$ The same authors reported an interesting coupling that involves a C-H activation of the non-fluorinated partner (Scheme $76, \mathrm{~L}=$ diphosphine) ${ }^{236}$ In this case a 2-pyridinyl group as directing group was more effective than the oxazoline used before. The reaction can be extended to other polyfluorinated aryls taking into account that the same selectivity and reactivity limitations on the number of fluorine atoms mentioned for the Suzuki couplings apply. No kinetic isotope effect was observed in the reaction and this, along with independent experiments that showed that the transmetalation-reductive elimination steps are facile, led to conclude that the C-F oxidative addition step is rate limiting. 


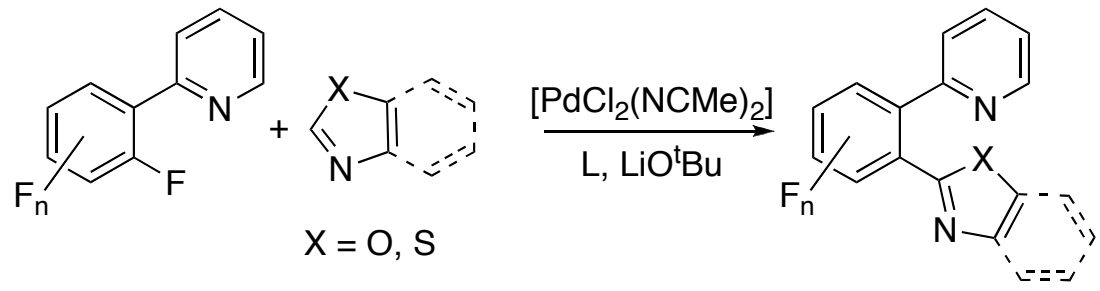

Scheme 76

The Stille reaction allows the introduction of groups different from aryls, and the catalytic vinylation of pentafluorophenyl pyridine has been reported (Scheme 77). The catalyst used, $\left[\mathrm{Pd}\left(\mathrm{C}_{5} \mathrm{~F}_{4} \mathrm{~N}\right) \mathrm{FL}_{2}\right]$, is the product of oxidative addition of the fluorinated pyridine to $\operatorname{Pd}(0)$. The involvement of this complex in the actual catalytic cycle is supported by the stoichiometric reaction of $\mathrm{SnBu}_{3}\left(\right.$ vinyl) and $\left[\mathrm{Pd}\left(\mathrm{C}_{5} \mathrm{~F}_{4} \mathrm{~N}\right) \mathrm{FL}_{2}\right]$ which leads to the vinyl pyridine product. ${ }^{237}$

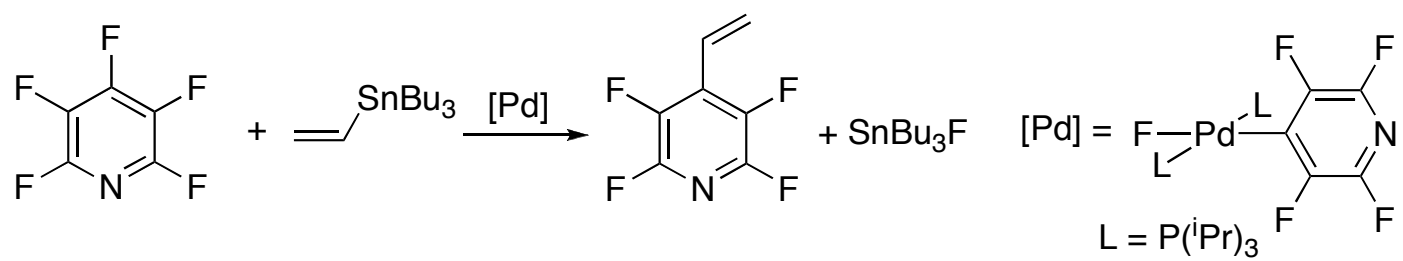

Scheme 77

Para-substituted aryl tetrafluoropyridines have been synthesized by the Suzuki coupling of pentafluoropyridine and arylboronic acids using the same type of catalyst shown in Scheme $77\left(\mathrm{~L}=\mathrm{P}^{\mathrm{i}} \mathrm{Pr}_{2}\left(\mathrm{CH}_{2} \mathrm{CH}_{2} \mathrm{OCH}_{3}\right)\right)$. ${ }^{238}$

In contrast to the Suzuki and Stille processes described, electron withdrawing substitutents on the ring are not necessary for the coupling of fluoroaryls with Grignard reagents to occur, the so called Kumada-Corriu process. ${ }^{239}$ Although nickel catalysis is more common in these processes, palladium catalyzed reactions have also been reported. Monoarylation is selectively observed in polyfluorinated aryls for the catalytic system shown in Scheme 78; this is an advantage over the analogous nickel catalysts 
since this metal invariably leads to di or even trisubstituted derivatives along with the monoarylated compound. ${ }^{240}$ The reaction shown in Scheme 79 works well when the ortho $\mathrm{X}$ group is a protic hydroxo or amino group acting as a directing group. Interestingly the C-F activation occurs even if a chloro substituent is present $(\mathrm{Y}=$ Cl). ${ }^{241}$ No mechanistic rationale has been given for these processes.

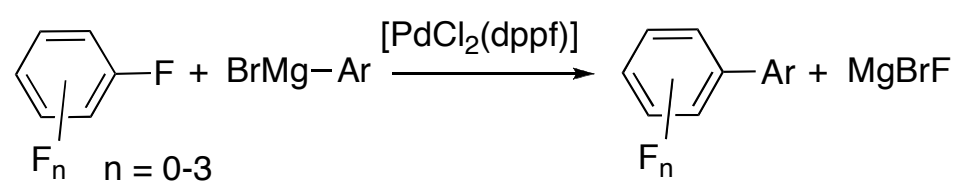

Scheme 78

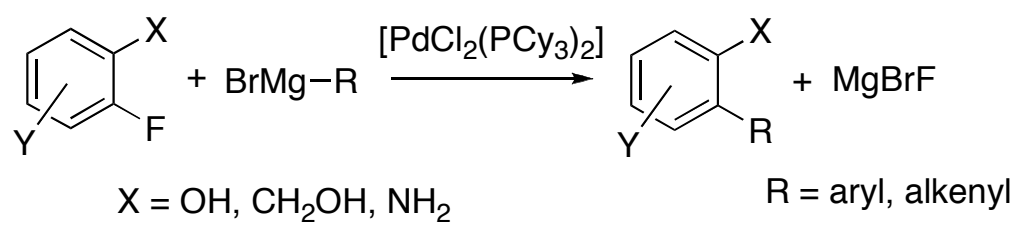

Scheme 79

The reaction of $\mathrm{C}_{6} \mathrm{~F}_{5} \mathrm{H}$ with phenols catalyzed by a mixture of $\mathrm{Pd}(\mathrm{OAc})_{2}$ and a silver salt leads to tetrafluoro diaryl ethers (Scheme 80$){ }^{242}$ This can be described as a palladium mediated formal nucleophilic substitution of the fluorine in the para-position although a mechanistic study was not carried out. Similar nucleophilic substitution on the activated para C-F bond of pentafluoropyridine, ${ }^{243}$ or on the unactivated site of a monofluoroquinoline have been also reported in stoichiometric processes. ${ }^{244}$<smiles>[R]c1ccc(O)cc1</smiles>

Scheme 80 


\subsubsection{C-C Coupling reactions of fluorinated alkenes.}

C-F bond activation of perfluorinated alkenes by $\left[\mathrm{Pd}\left(\mathrm{PR}_{3}\right)_{n}\right]$ complexes has been reported. The oxidative addition of a coordinated tetrafluoroethylene to $\operatorname{Pd}(0)$ leads to alkenyl palladium fluoride complexes which have been isolated and structurally characterized. ${ }^{245,246}$ This oxidative addition is promoted by the addition of LiI or Lewis acids such as boranes and this has been used to functionalize $\mathrm{C}_{2} \mathrm{~F}_{4}$ in a catalytic manner (Scheme 81). Thus, the synthesis of trifluoro styrene and other trifluorovinyl arenes has been carried out by the Negishi coupling of $\mathrm{C}_{2} \mathrm{~F}_{4}$ with $\mathrm{ZnAr}_{2} .{ }^{245}$ The Suzuki coupling of $\mathrm{C}_{2} \mathrm{~F}_{4}$ with arylboronates can be carried out without the addition of a base, the fluorine resulting in the $\mathrm{C}-\mathrm{F}$ cleavage, coordinating to boron and acting as a transmetalation promoter. $^{247}$

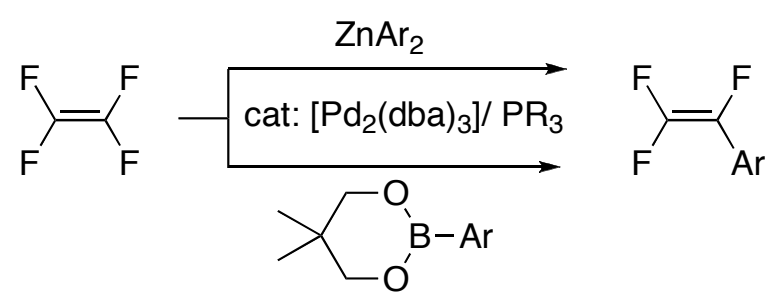

Scheme 81

$\beta$-F elimination reactions have been proposed to occur in several catalytic transformations of fluoroalkenes, such as the Heck reaction of 1,1-difluoroethylene to give monofluorostyrene, ${ }^{248}$ or intramolecular cyclizations that involve C-F cleavage in a putative palladium $\beta$-difluoroalkyl (Scheme 82 , a). ${ }^{249,250}$ A similar 5-endo-trig cyclization was observed starting from a trifluoromethylallyl derivative (Scheme 82, b). Again $\beta$-F elimination in the intermediate palladium cycloalkyl complex explains the formation of the difluoromethylene substituted pyrroline. ${ }^{251}$ 
a)

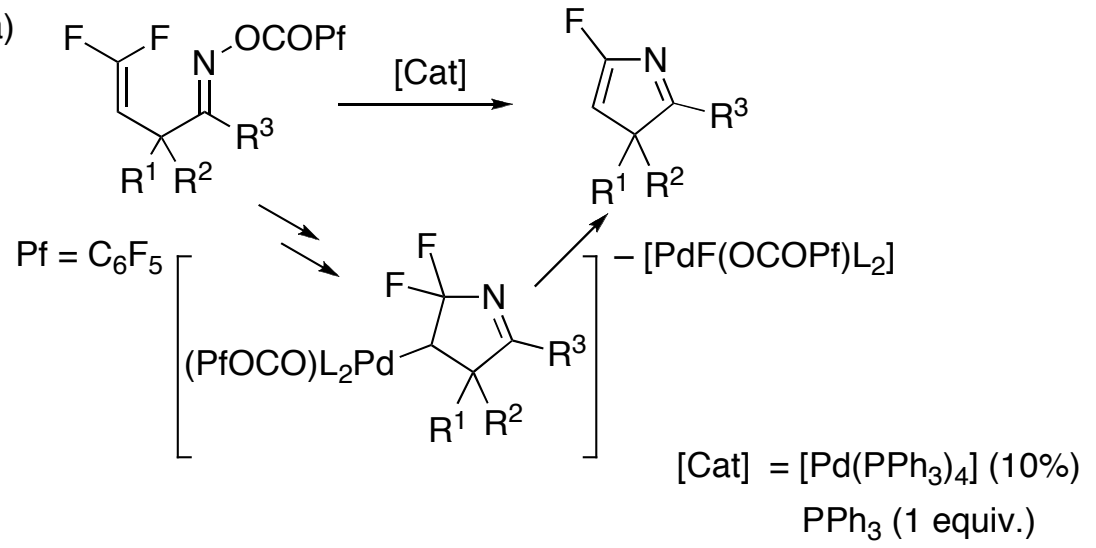

b)

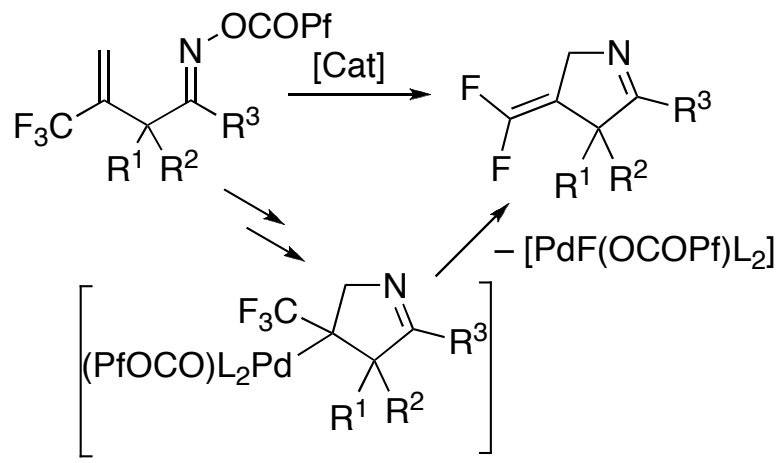

Scheme 82

\subsubsection{Allylic Substitutions of a fluorine atom.}

Few catalytic processes of this type have been reported and the use of simple allylic monofluorides leads to non-fluorinated compounds. ${ }^{252}$ However, using 3,3difluoropropenes as reagents, Paquin et al. described the palladium catalyzed allylic substitution of a fluoride by an amino group which allows to synthesize monofluorinated amines (Scheme 83). ${ }^{253}$

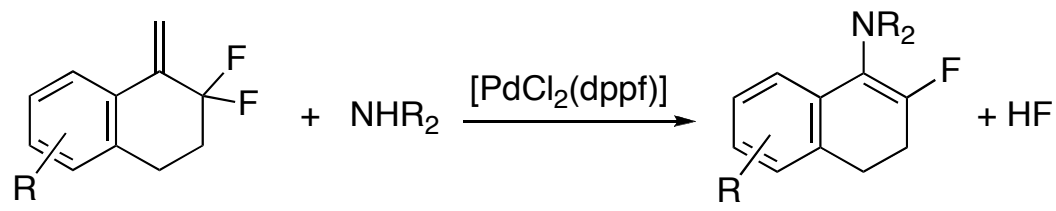

Scheme 83 


\subsubsection{Hydrodefluorination reactions.}

This topic has been reviewed recently covering all metal-catalyzed processes, ${ }^{254}$ but just palladium mediated reactions will be discussed here. The replacement of fluorine by hydrogen is a reaction that has been observed in the catalytic hydrogenation of allylic, vinylic, benzylic and aromatic fluorides using heterogeneous palladium catalysts such as Pd on carbon. ${ }^{255} \mathrm{C}_{6} \mathrm{H}_{5} \mathrm{~F}$ can be hydrodefluorinated to benzene using 2propanol $/ \mathrm{NaOH}$ as reagent and an heterogeneous catalyst formed by $\mathrm{Pd}$ on carbon. Among the halobenzenes $\mathrm{C}_{6} \mathrm{H}_{5} \mathrm{X}(\mathrm{X}=\mathrm{F}, \mathrm{Cl}, \mathrm{Br}, \mathrm{I})$ fluorobenzene is the most reluctant one to be dehalogenated and this is clearly shown in a competitive experiment using a mixture of all the $\mathrm{C}_{6} \mathrm{H}_{5} \mathrm{X}$ compounds: $\mathrm{C}_{6} \mathrm{H}_{5} \mathrm{~F}$ reacts only when the other halides have been consumed. ${ }^{256}$ Fluorobenzene has also been hydrodefluorinated using hydrogen on a $\mathrm{Pd}-\mathrm{SiO}_{2}$ heterogeneous catalyst where a rhodium pyridyl complex has been tethered. ${ }^{257}$

Catalytic hydrodefluorination of fluorobenzene and substituted fluoroarenes has been carried out with a heterometallic homogeneous $\mathrm{Pd}-\mathrm{Ru}$ catalyst using isopropanol and a base as a hydrogen source (Scheme 84$).{ }^{258}$ The presence of both metals in the same complex is needed for an efficient catalysis and neither the homonuclear palladium or rhodium analogue dimers nor a mixture of both homonuclear complexes have the high activity observed for the heteronuclear complex. The system is also capable of complete hydrodefluorination of benzylic trifluoromethyl substituents (Scheme 84). When the organic fluoride has aromatic and benzylic C-F bonds, such as in 4-fluoro benzotrifluoride, the aromatic C-F bonds are substituted first. 

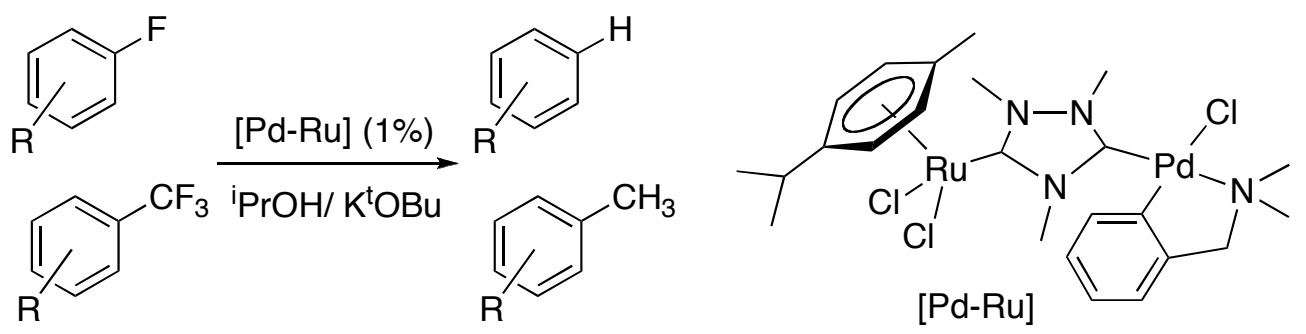

Scheme 84

Hydrodefluorination reactions of perfluoroarenes are very interesting for the synthesis of partially fluorinated derivatives. Selective substitution of the para fluorine by hydrogen in pentafluoropyridine has been carried our catalytically using a borane as a hydrogen source (HBpinacol) ${ }^{238}$ The catalyst employed is $\left[\mathrm{PdF}\left(\mathrm{F}_{4}-\mathrm{py}\right) \mathrm{L}_{2}\right]$ (Scheme 85, $\mathrm{L}=\mathrm{P}^{\mathrm{i}} \mathrm{Pr}_{3}$ or $\left.\mathrm{P}^{\mathrm{i}} \mathrm{Pr}_{2}\left(\mathrm{CH}_{2} \mathrm{CH}_{2} \mathrm{OMe}\right)\right)$ and this is the actual product of oxidative addition of perfluoropyridine to $\operatorname{Pd}(0)$. Treatment of that complex, $\left[\mathrm{PdF}\left(\mathrm{F}_{4}-\mathrm{py}\right) \mathrm{L}_{2}\right]$, with silane leads to the hydrido derivative $\left[\mathrm{PdH}\left(\mathrm{F}_{4}-\mathrm{py}\right) \mathrm{L}_{2}\right]$ which in turn undergoes reductive elimination to give tetrafluoropyridine (Scheme 85). ${ }^{259}$ These stoichiometric experiments support that the catalytic hydrodefluorination reaction occurs in the same way.

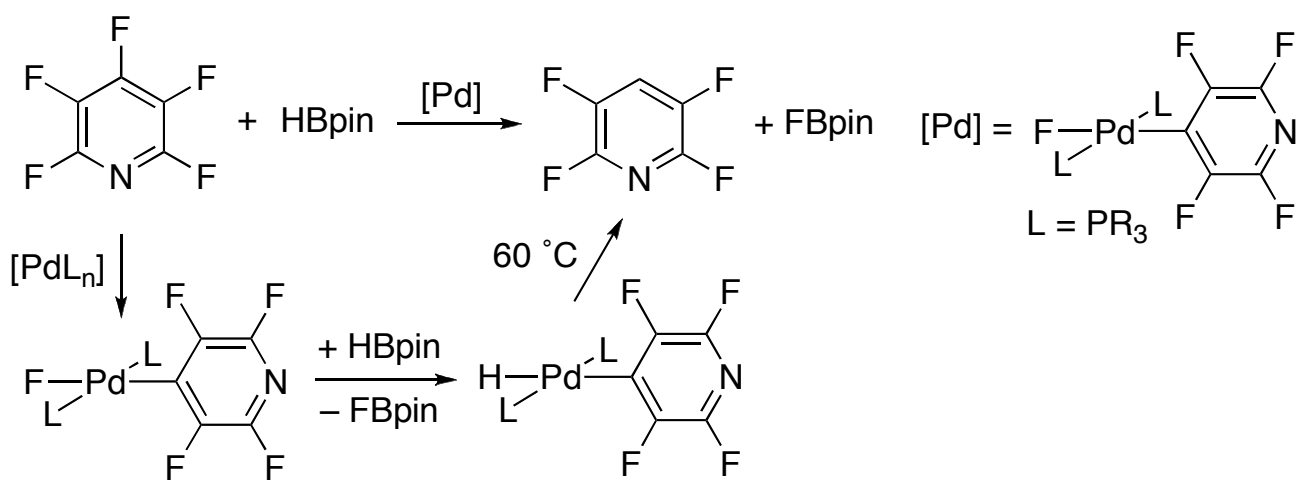

Scheme 85

Following the same strategy already employed in $\mathrm{C}-\mathrm{C}$ coupling reactions, Zhang et al. used 2-pyridyl as a directing group and observed the selective hydrodefluorination 
of the fluorine atoms ortho to the directing group in 2-pyridylpolyfluorobenzenes. ${ }^{260}$ The reaction of these derivatives with silanes led to selective ortho monohydrodefluorination (Scheme 86). By increasing the reaction temperature to 120 ${ }^{\circ} \mathrm{C}$ and the amount of silane, hydrodefluorination of both ortho fluorine atoms took place. Stoichiometric reactions of 2-pyridylperfluorobenzene and a $\operatorname{Pd}(0)$ complex showed selective oxidative addition of one ortho C-F bond, which drives the regiochemistry of the reduction process.

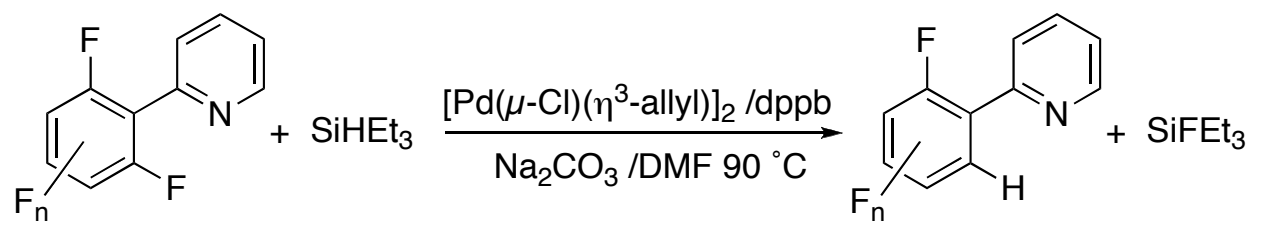

Scheme 86

Monofluorinated alkenes have been synthesized by hydrodefluorination of gemdifluoro allyls. A silane was used as a hydrogen source and a base is also necessary. Independent experiments showed that $\mathrm{SiH}_{3} \mathrm{Ph}$ is not very reactive in contrast to $\mathrm{SiH}_{2} \mathrm{Ph}(\mathrm{OEt})$ or $\mathrm{SiHPh}(\mathrm{OEt})_{2}$ which can be formed in the reaction medium $(\mathrm{EtOH})$ in the presence of a base $\left(\mathrm{NEt}_{3}\right)$. The reaction is regioselective and deuteration experiments showed that the hydrogen is incorporated in the allylic position and silane, not the solvent, is the H source (Scheme 87). ${ }^{261}$

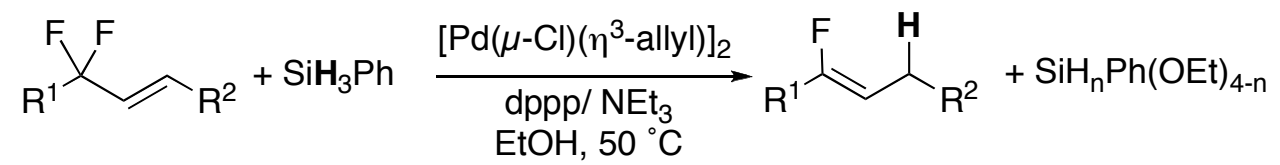

Scheme 87 


\subsection{Overview of catalytic C-F forming reactions.}

The palladium catalyzed fluorination is a fast growing field and the reactions that have been reported so far can be classified in two major groups according to the fluorine source used, which in turn usually implies quite a different mechanistic pathway.

Recent advances in fluorination reactions have introduced compounds that are a source of fluorine acting as an electrophile. They are usually N-fluoro substituted ammonium salts or other aminoderivatives and they are powerful oxidizing agents. A few examples are shown in Figure 12 and some of them are commercially available. Many Pd-catalyzed fluorination reactions that use these compounds are believed to occur though a $\mathrm{Pd}(\mathrm{II}) / \mathrm{Pd}(\mathrm{IV})$ catalytic cycle.

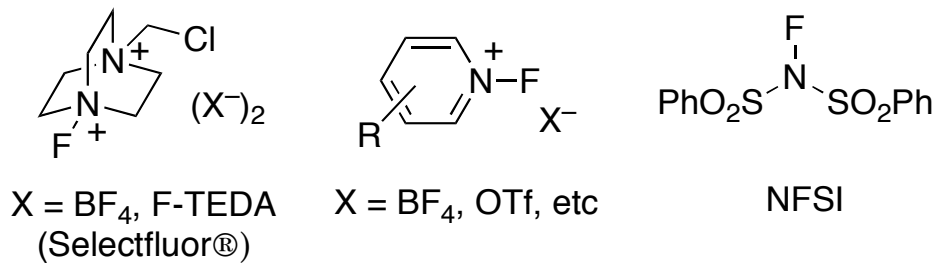

Figure 12. Electrophilic fluorinating reagents.

The second group of reactions use fluoride salts as the $\mathrm{F}$ source, i.e. a nucleophilic fluoride. These are more attractive as far as availability of the reagents is concerned and, generally, a $\operatorname{Pd}(0) / \operatorname{Pd}(\mathrm{II})$ catalytic cycle seems to operate.

\subsubsection{Pd-catalyzed fluorination with electrophilic fluorine sources.}

The activation of a C-H bond of enol-type compounds followed by the enantioselective substitution by fluorine has been reported for different substrates. Catalytic asymmetric fluorination of $\beta$-keto esters has been achieved using chiral 
diphoshino hydroxo or aquo palladium(II) complexes (Scheme 88). The source of fluorine is the electrophilic reagent $N$-fluorobenzenesulfonimide (NFSI). Very good yields and enantiomeric excess were obtained. ${ }^{262,263}$ The same reaction was performed using ionic liquids as solvents and this method allowed to recycle the catalyst at least 10 times, the yields and enantioselectivity remaining high throughout the reusing experiments. $^{264}$

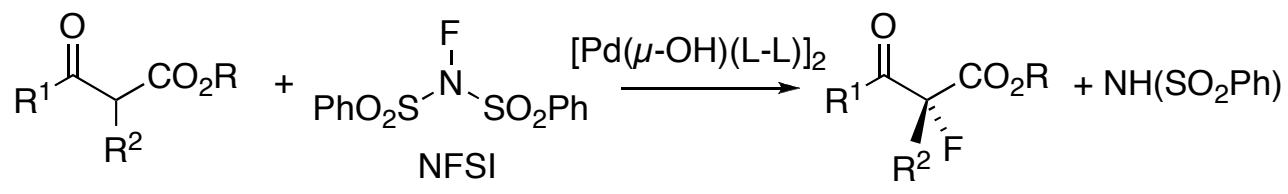<smiles>[R16]c1ccc2ccccc2c1-c1c(P)ccc2ccccc12</smiles>

(R)-BINAP

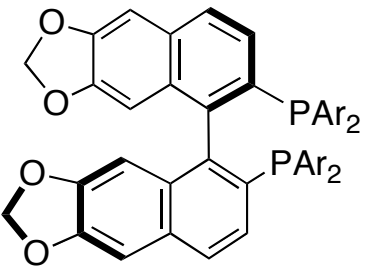

(R)-SEGPHOS

$84-91 \%$ ee

Scheme 88

Close related catalytic systems have been reported for the enantioselective synthesis of fluorinated $\beta$-keto phosphonates, ${ }^{263,265}$ and $\alpha$-cyano acetates. ${ }^{266}$ Lectka et al. have developed the enantioselective $\alpha$-fluorination of acyl chlorides that lead, by reaction with suitable nucleophiles, to a variety of $\alpha$-fluorinated carbonyl compounds. A combination of a cinchona alkaloid catalyst, a base, NFSI and $\left[\mathrm{PdCl}_{2}\left(\mathrm{PPh}_{3}\right)_{2}\right]$ bring about the reaction and it is proposed that the role of the palladium complex is just the coordination of the enolate formed, without actually participating in the C-F bond formation. $^{267}$

Activation of an aromatic or benzylic $\mathrm{C}-\mathrm{H}$ bond is more difficult than $\mathrm{C}-\mathrm{H}$ activation leading to an enolate, but the substitution of a $\mathrm{C}-\mathrm{H}$ bond in these reagents by a fluoride has been achieved by Sanford et al. using electrophilic fluorine sources. $\mathrm{N}$ - 
fluoro-2,4,6-trimethylpyridinium tetrafluoroborate turned out to be the best reagent for the benzylic fluorination of 8-methylquinoline derivatives whereas $\mathrm{N}$-fluoropyridinium tetrafluoroborate is the reagent of choice in the fluorination of 2-aryl pyridine derivatives (Scheme 89). ${ }^{268}$ In both cases a coordinating directing group (the heterocyclic nitrogen atom) is present and the reaction selectively occurs on the ortho position of the aryl or the benzyl group in 8-methyl quinolines. When both benzylic and aromatic fluorination is possible in the same substrate, aryl fluorination is preferred.

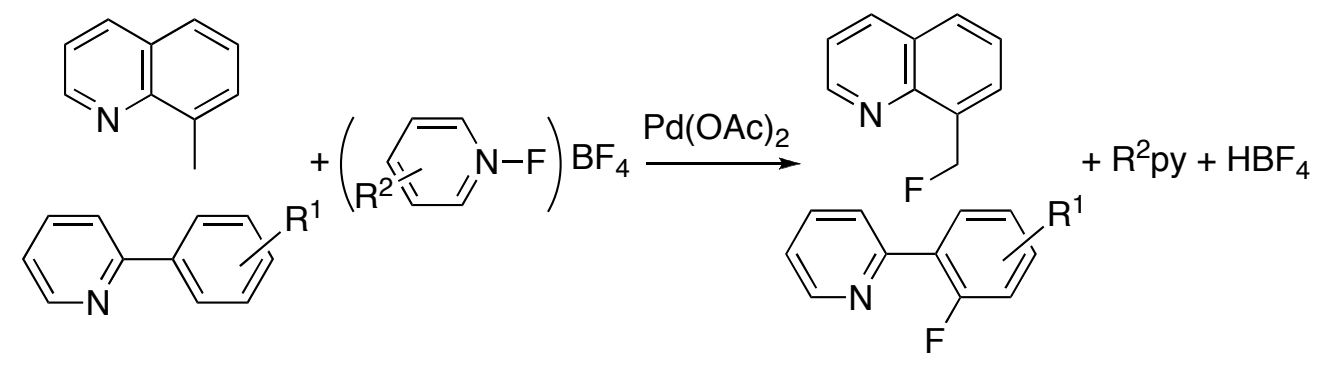

Scheme 89

$\mathrm{Yu}$ et al. also achieved the ortho fluorination of aryls bearing a triflamide substituent as a directing group to facilitate orthometalation to the metal, i.e. C-H cleavage and formation of the Pd-aryl bond. After screening, $\operatorname{Pd}(\mathrm{OTf})_{2}$ was the best catalysts and the electrophilic fluorine source of choice was $N$-fluoro-2,4,6trimethylpyridinium tetrafluoroborate (Scheme 90, a) ${ }^{269}$ Careful optimization of the reaction conditions led to the synthesis of ortho mono and difluorinated compounds. The authors showed that the triflamide product can be transformed in a variety of organic fluorinated derivatives. A further development by the same group is the synthesis of ortho monofluorinated carboxylic acids using an amide as ortho directing groups (Scheme 90, b). ${ }^{270}$ 
a)

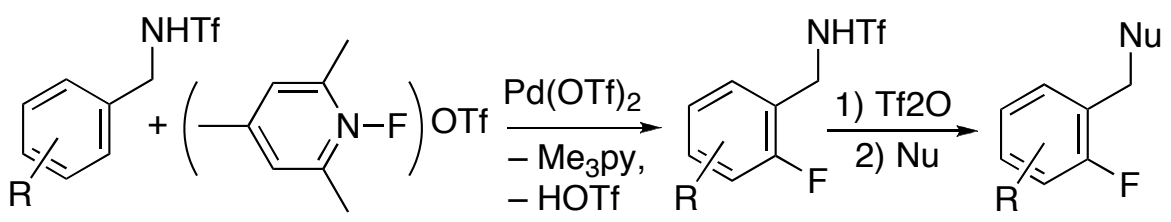

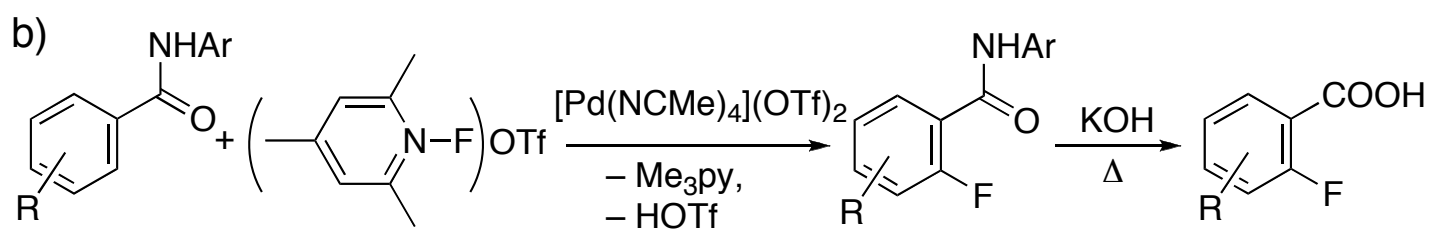

Scheme 90

A different approach to the synthesis of aryl fluorides is the conversion of aryl boronic acids and related derivatives into aryl fluorides and this has been carried out both in a stoichiometric and in a catalytic way. The reaction of a boronic acid with a stoichiometric amount of a $\mathrm{Pd}(\mathrm{II})$ complex bearing a chelating $\mathrm{N}$-donor ligand leads to an aryl palladium(II) complex. In a second step, this complex reacts with Selectfluor to give the final ArF product (Scheme 91). ${ }^{271}$ The reaction occurs through the formation of a $\operatorname{Pd}(\mathrm{IV})$ aryl-fluoro complex which undergoes reductive elimination of ArF. These steps have been supported by the isolation of relevant intermediates and the study of individual steps. ${ }^{272}$

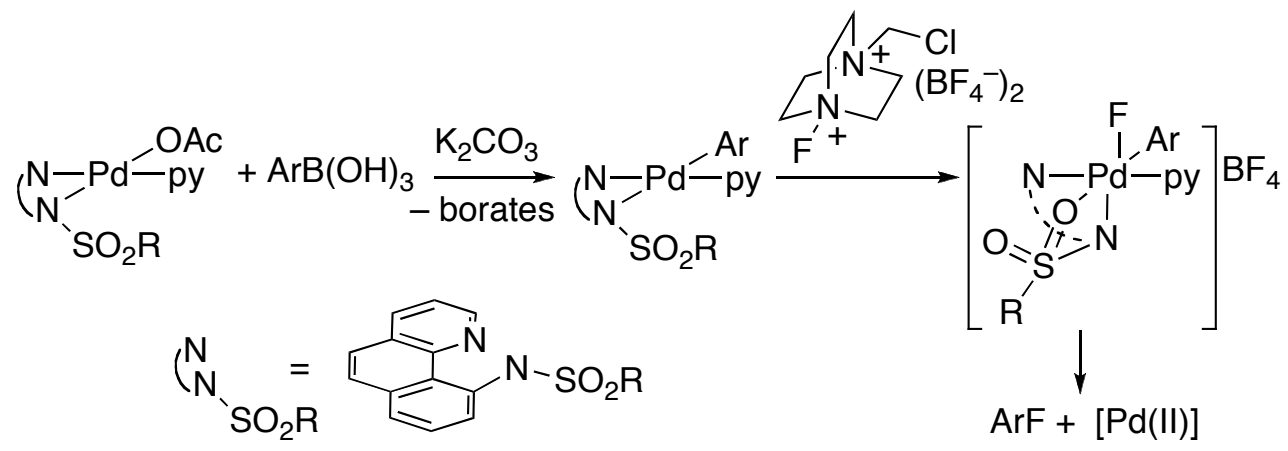

Scheme 91 
Recently, the Pd-catalyzed conversion of boronic acid derivatives, mainly aryl trifluoroborates, into $\mathrm{ArF}$ has been described. ${ }^{273}$ The reaction uses a mixture of Selectfluor and $\mathrm{NaF}$, but isotope labeling studies using ${ }^{18} \mathrm{~F}$ have demonstrated that the fluorine atom in the product originates in the electrophilic fluorine reagent (Scheme 92). Interestingly, the reaction is mechanistically distinct and consistent with the operation of a radical pathway. A single electron transfer oxidation of the palladium complex by Selectfluor occurs to form a Pd(III) derivative. Subsequent $\mathrm{F}$ transfer from the generated Selectfluor radical cation to the aryl borate occurs.

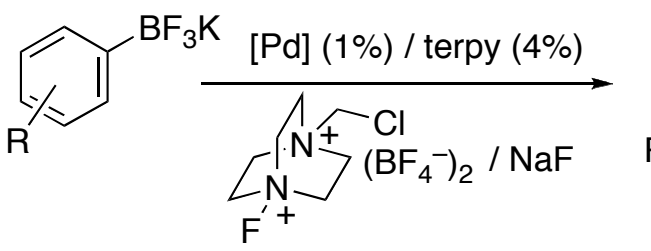<smiles>[R]c1ccc(F)cc1</smiles>
$[\mathrm{Pd}]=$

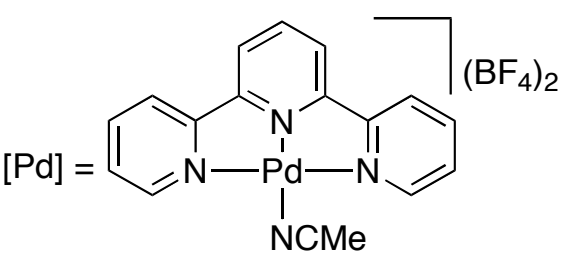

Scheme 92

Liu's group has developed several processes to fluorinate alkene and alkyne substrates. The tandem fluorination and cyclization of enynes leads to cyclic derivatives with exocyclic fluoroalkenes (Scheme 93). ${ }^{274}$ The catalyst used is a mixture of palladium trifluoroacetate and the $\mathrm{N}$-donor chelating ligand bathocuproine and $\mathrm{N}$ fluorobenzenesulfonimide (NFSI) was used as the fluorine source.

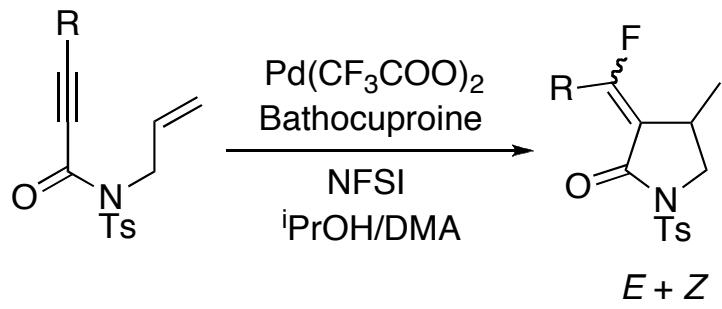<smiles>Cc1cc(-c2ccccc2)c2ccc3c(-c4ccccc4)cc(C)nc3c2n1</smiles>

Bathocuproine

Scheme 93 
The same catalyst system was used in the reaction with styrenes and NFSI. However, in this case the aminofluorination of the double bond was observed and both fluoro and amino groups of the electrophilic reagent end up on the final product (Scheme 94, a). ${ }^{275}$ The intramolecular version of this aminofluorination reaction has also been developed but in this case the fluorine source is $\mathrm{AgF}$, which can be considered a nucleophilic fluorine source. However, a strong oxidant, $\mathrm{PhI}(\mathrm{OCOR})_{2}$, is also added to the reaction mixture so both in the intra and the intermolecular reactions the intermediacy of Pd(IV) species were proposed (Scheme 94, b). ${ }^{276}$

a)

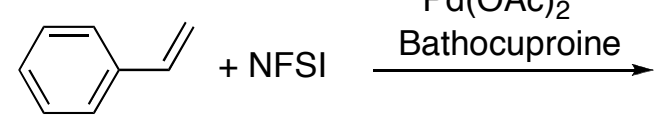

b)<smiles>[R]C([R])(CC=C)CN[SnH3]</smiles>

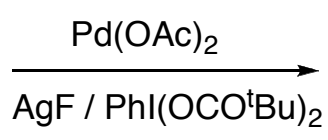

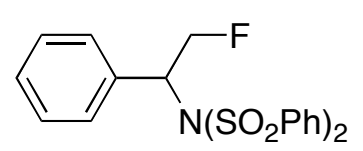<smiles>[Y5]N1C[C@@H](F)CC([R])([R])C1</smiles>

Scheme 94

It is worth noting that electrophilic fluorine reagents have been used as oxidants in palladium catalyzed processes where they do not act a fluorinating agents. In most of these cases a $\mathrm{Pd}(\mathrm{II}) / \mathrm{Pd}(\mathrm{IV})$ mechanism is often invoked. ${ }^{277}$

\subsubsection{Pd-catalyzed fluorination with nucleophilic fluorine sources.}

Nucleophilic fluorine sources are more attractive than electrophilic fluoro reagents since they are more accessible, less expensive and the corresponding ${ }^{18} \mathrm{~F}$ derivatives are available. This is important since the synthesis of ${ }^{18} \mathrm{~F}$ labeled compounds, to be used as contrast agents in positron emission tomography (PET), requires fast preparation methods for reagents and products considering the short ${ }^{18} \mathrm{~F}$ half life $\left(\mathrm{t}_{1 / 2}=110 \mathrm{~min}\right)$. 
This is not always possible if electrophilic fluorine reagents need to be used.

The synthesis of aryl fluorides using MF sources is challenging and not many processes have been reported. Although the Pd-catalyzed formation of $\mathrm{C}-\mathrm{X}$ bonds is a well established process $\left(\mathrm{X}=\mathrm{OR}, \mathrm{NR}_{2}, \mathrm{SR}\right.$, etc. $),{ }^{278}$ and the operation of a similar catalytic mechanism could be envisaged for $\mathrm{X}=\mathrm{F}$, the $\mathrm{C}-\mathrm{F}$ bond formation process is sluggish. This has been attributed to a reluctant $\mathrm{C}-\mathrm{F}$ reductive elimination process, as it is discussed below. Notwithstanding this difficulty, Buchwald et al. have developed the synthesis of aryl fluorides from aryl triflates and CsF catalyzed by a palladium precursor complex and BrettPhos ligands (Scheme 95). ${ }^{279}$ The reaction has a considerably large scope and works well with heterocycles and different aryls, specially those bearing electron withdrawing substitutents and non-donor ortho substituents. The process was further improved with the use of AdBrettPhos and a preformed $\left[\mathrm{Pd}_{2} \mathrm{~L}_{2}(\mu-\right.$ $\left.\left.\eta^{2}-\eta^{2}-\mathrm{COD}\right)\right]$ precatalyst $(\mathrm{L}=$ AdBrettPhos $) .{ }^{280} \mathrm{~A}$ nice development is the use of a microflow system consisting of a packed $\mathrm{CsF}$ bed reactor to carry out this reaction. Short residence times were enough to achieve good yields of the aryl fluorides. ${ }^{281}$

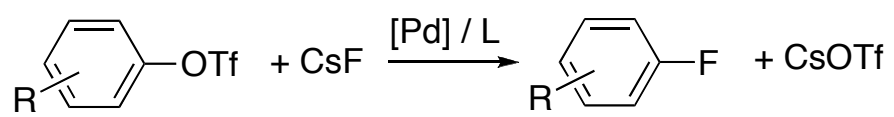

$[\mathrm{Pd}]=\left[\mathrm{Pd}\left(\mathrm{CH}_{2} \mathrm{TMS}\right)_{2}(\mathrm{COD})\right]$ or $[\mathrm{Pd}(\mu-\mathrm{Cl})(\text { cinnamyl })]_{2}$<smiles>COc1ccc(OC)c(-c2c(P)cc(C(C)C)cc2P(C)C)c1P</smiles>

$\mathrm{R}=\mathrm{Cy}$, BrettPhos

$\mathrm{R}={ }^{\mathrm{t}} \mathrm{Bu},{ }^{\mathrm{t}} \mathrm{BuBrettPhos}$

$\mathrm{R}=\mathrm{Ad}, \mathrm{AdBrettPhos}$

Scheme 95

A conventional $\mathrm{Pd}(0) / \mathrm{Pd}(\mathrm{II})$ catalytic cycle involving oxidative addition of the aryltriflate, coordination of the fluoride and reductive ArF elimination seems to be at work in this reaction. This is supported by stoichiometric experiments which have 
shown that an isolated $\left[\mathrm{Pd}\left(\mathrm{Ar}_{\mathrm{EWG}}\right) \mathrm{FL}\right]$ complex $\left(\mathrm{L}=\right.$ Brettphos, $\operatorname{Ar}_{\mathrm{EWG}}=$ aryl with electron withdrawing groups) leads to the reductive elimination product ArF albeit in low yields. Subsequent studies by the same authors involving aryl groups with electron donating substituents have shown that in this case $\left[\mathrm{Pd}\left(\mathrm{Ar}_{\mathrm{EDG}}\right) \mathrm{FL}\right]$ compounds do not undergo reductive elimination of ArF. They have also observed that other competing processes are operating in the catalytic system including the arylation of the phosphine ligand in the presence of base. This consumes part of the ArOTf reagent and generates a slightly different ligand that nonetheless is more effective and capable of $\mathrm{ArF}$ formation. ${ }^{282}$ The information gathered in these studies have led to a design of a modified catalytic system that can transform aryl bromides, a more available and common type of aryl derivatives, into aryl fluorides (Scheme 96). The use of a combination of $\mathrm{AgF}$ and $\mathrm{KF}$ gives good results, the former facilitating the coordination of fluoride to palladium and the later acting as a base to induce the arylation of the added BrettPhos ligand. In the most reluctant cases, such as the fluorination of pyridine bromide derivatives, the preformed arylated ligand $\left(\mathrm{L}_{\mathrm{Ar}}\right)$ can be used with good results. $^{283}$

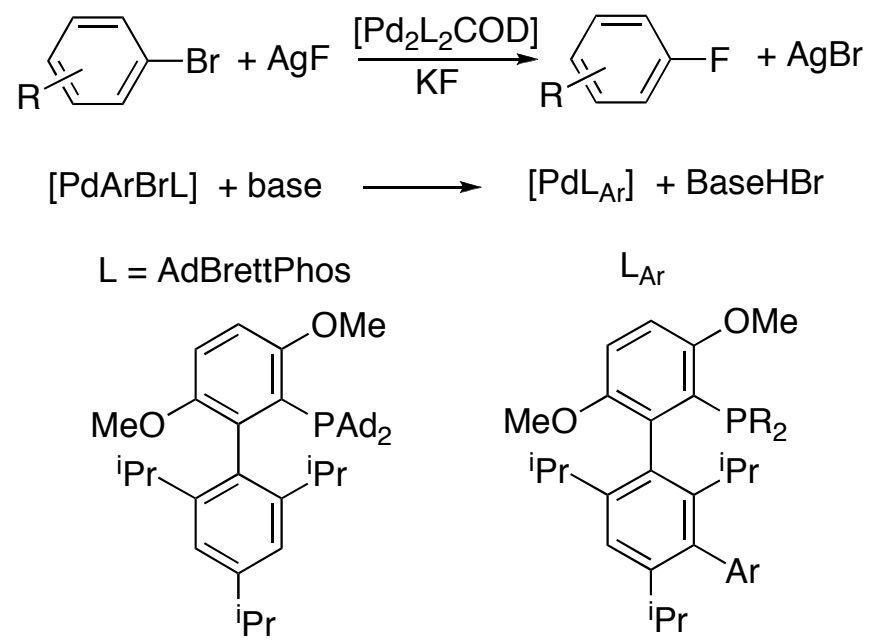

Scheme 96 
In a mixed approach, Ritter et al. developed the synthesis of aryl fluorides with electron donating groups using an electrophilic fluorine reagent, a palladium(IV) fluoro complex, synthesized from a fluoride salt. The synthesis is stoichiometric in palladium and it is oriented to obtain ${ }^{18} \mathrm{~F}$ labeled aryls for PET in short reaction times. Upon addition of the labeled fluoride to a dicationic $\operatorname{Pd}(\mathrm{IV})$ complex, this is transformed into a Pd(IV) fluoro complex (Scheme 97). This complex is capable of oxidatively transfer the fluorine atom to a $\mathrm{Pd}(\mathrm{II})$ aryl complex. The new aryl $\mathrm{Pd}(\mathrm{IV})$ fluoro compound undergoes reductive elimination to give the labeled aryl fluoride. ${ }^{284}$

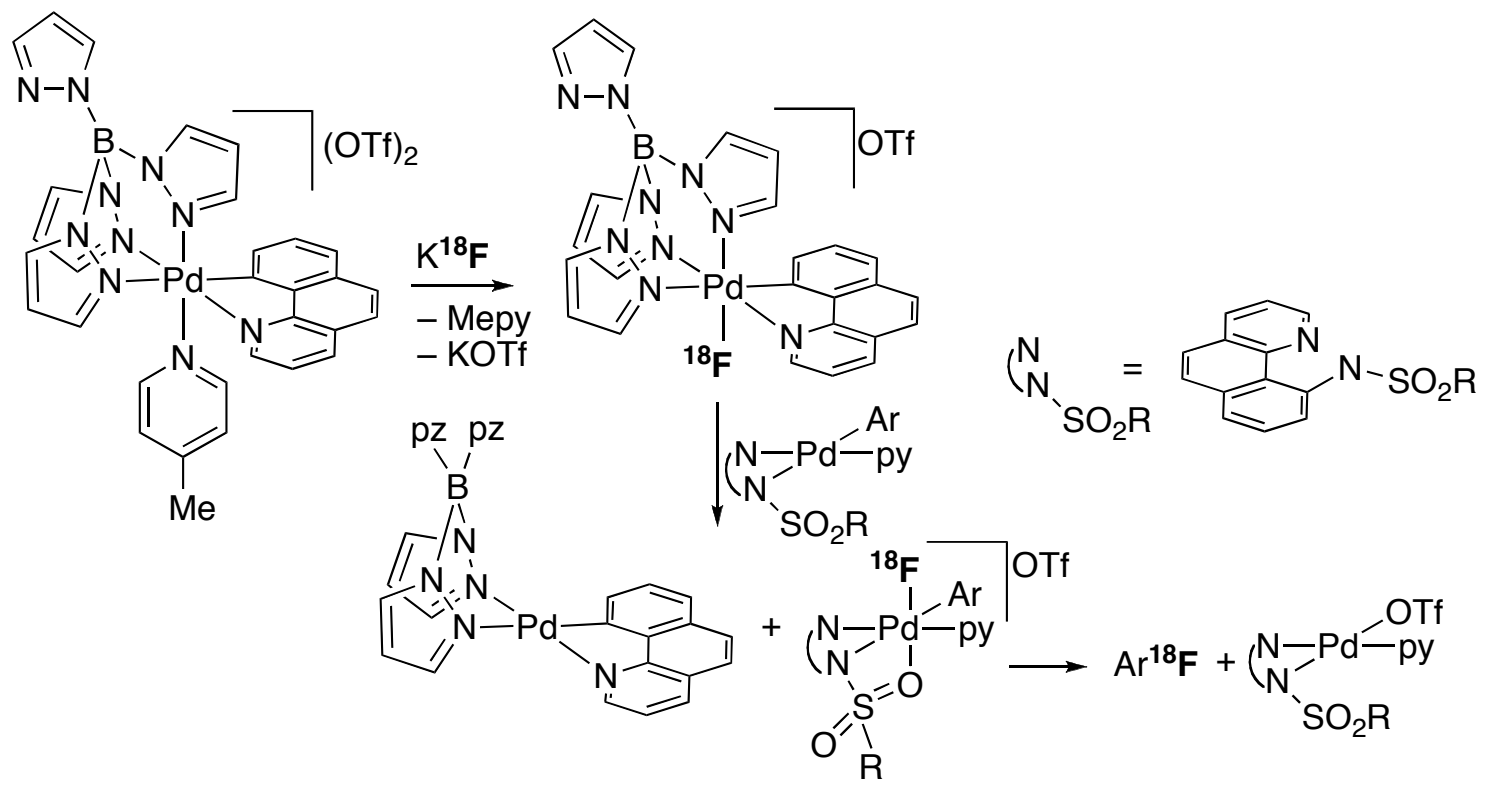

Scheme 97

Fluorination reactions of other organic derivatives different from aryl halides or triflates with fluoride salts rely on the nucleophilic attack of $\mathrm{F}^{-}$on an organometallic palladium complex. One of the early reports of this type of reaction is the synthesis of acid fluorides by carbonylation of organic halides followed by nucleophilic attack on the palladium acyl moiety (Scheme 98). ${ }^{285}$ 


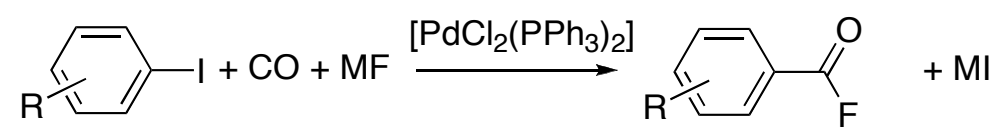

Scheme 98

The nucleophilic attack on a palladium allyl complex is a well known reaction and it has been extended to fluoride as the nucleophile. Gouverneur, Brown et al. have reported the reaction of allylic carbonates with a fluoride salt to give allylic fluorides in good yields, including the ${ }^{18} \mathrm{~F}$ labeled derivatives. In this report the authors use only allylic substrates that cannot give $\beta-\mathrm{H}$ elimination, a competitive reaction often observed in the presence of fluoride (Scheme 99). As it is expected for a Pd-mediated allylic nucleophilic substitution, the reaction occurs by oxidative addition of the allylic carbonate to $\operatorname{Pd}(0)$ and nucleophilic attack of fluoride on the resulting palladium allylic complex. $^{286}$

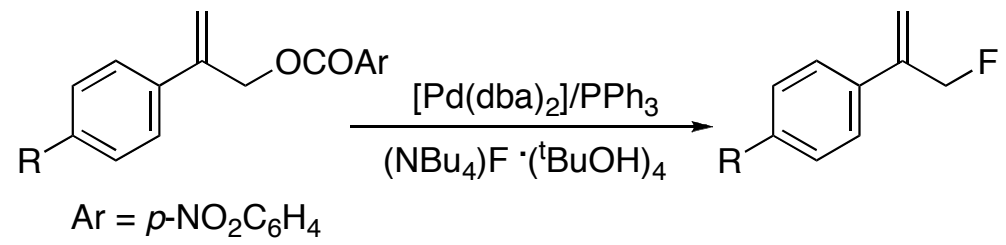

Scheme 99

Doyle's group has developed the enantioselective allylic fluorination reaction of a variety of cyclic and acyclic allyls with silver fluoride. ${ }^{287,288}$ The analysis of the structure of the fluorinated cyclic allyls shows that the fluoride attack is, as expected, trans to palladium. ${ }^{287}$ In the case of non-cyclic reagents, with the exception of the cynnamyl derivatives $(\mathrm{R}=\mathrm{Ph})$, the internal fluoride is the preferred regioisomer which has been enantioselectively obtained using Trost's diphosphines (Scheme 100). ${ }^{288}$ 


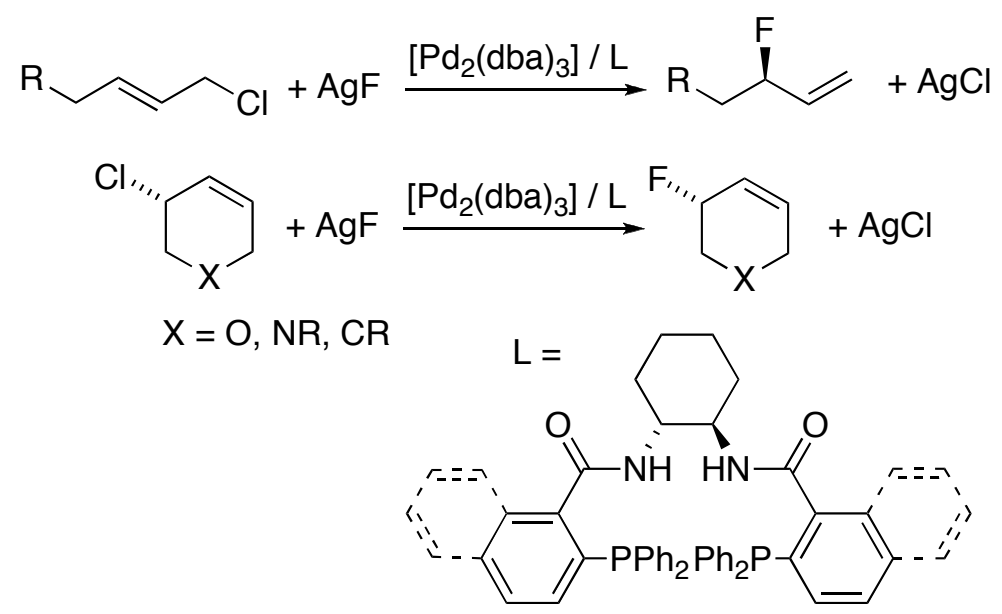

Scheme 100

\subsection{The Pd-F bond}

Isolated and well characterized palladium(II) fluoro complexes are relatively recent. Grushin et al. synthesized $\left[\mathrm{PdRF}\left(\mathrm{PPh}_{3}\right)_{2}\right]$ by halogen metathesis of the analogous iodo complex with $\mathrm{AgF}$ or, alternatively, by protonation of a hydroxo derivative with $\mathrm{NEt}_{3} / 3 \mathrm{HF}$ (Scheme 101). ${ }^{289}$ These are the two most general methods for the preparation of monofluoro palladium organometallic derivatives. ${ }^{290,291}$ Since this first report several monomeric palladium fluoro complexes have been synthesized in the context of the study of C-F activation or C-F formation. Some of them have been already mentioned in this chapter or will be referred to below. Palladium difluoro derivatives are also known, ${ }^{292}$ as well as dimeric complexes with one, ${ }^{293}$ or two bridging fluorides. ${ }^{294-296}$

$$
\begin{gathered}
{\left[\mathrm{PdArl}\left(\mathrm{PPh}_{3}\right)_{2}\right]+\mathrm{AgF} \underset{\text { Ultrasound }}{\longrightarrow}\left[\mathrm{PdArF}\left(\mathrm{PPh}_{3}\right)_{2}\right]+\mathrm{Agl}} \\
3\left[\mathrm{Pd}(\mu-\mathrm{OH}) \mathrm{R}\left(\mathrm{PPh}_{3}\right)\right]_{2}+2 \mathrm{NEt}_{3}(\mathrm{HF})_{3} \longrightarrow 6\left[\mathrm{PdRF}\left(\mathrm{PPh}_{3}\right)_{2}\right]+2 \mathrm{NEt}_{3}+6 \mathrm{H}_{2} \mathrm{O} \\
\mathrm{R}=\mathrm{Me}, \mathrm{Ph}
\end{gathered}
$$

Scheme 101 
When coordinated to palladium the fluorine atom is a weak $\sigma$-donor and a strong $\pi$-donor ligand. Careful studies have been carried out on isolated palladium(II) fluoro complexes analyzing the structural and spectroscopic parameters that are sensitive to electronic factors in order to confirm the abovementioned ligand features. By comparing the Pd-C(aryl) bond length in the molecular structures of a series of trans$\left[\mathrm{PdPhX}\left(\mathrm{PPh}_{3}\right)_{2}\right]$ complexes $(\mathrm{X}=\mathrm{F}, \mathrm{Cl}, \mathrm{Br}, \mathrm{I}$, Figure 13, a), Grushin et al. have determined that the fluoro ligand shows the lowest trans influence of all the halogens, ${ }^{297}$ which is consistent with its being the weakest $\sigma$-donor. Cámpora et al. analyzed the ${ }^{13} \mathrm{C}$ NMR chemical shift variation upon coordination (coordination shift) of the $C_{i p s o}$ in a series of pincer $\mathrm{Pd}(\mathrm{II})$ complexes $\left[\left({ }^{\mathrm{iPr}} \mathrm{PCP}\right) \mathrm{PdX}\right] \quad\left({ }^{\mathrm{iPr}} \mathrm{PCP}=2,6\right.$-bis(diisopropylphosphinomethyl)phenyl, $\mathrm{X}=$ anionic ligand) (Figure 13, b). The values found correlate with the $\sigma$-donor ability of the ligand and the latter follow the order $\mathrm{X}$ : $\mathrm{OTf}<\mathrm{NO}_{3}<\mathrm{F}<\mathrm{OAc}<\mathrm{OMe}<\mathrm{Cl}<\mathrm{OH} \approx \mathrm{Br}<\mathrm{I}<\mathrm{NH}_{2}<\mathrm{Ph}<\mathrm{Me}<\mathrm{H}^{298}$
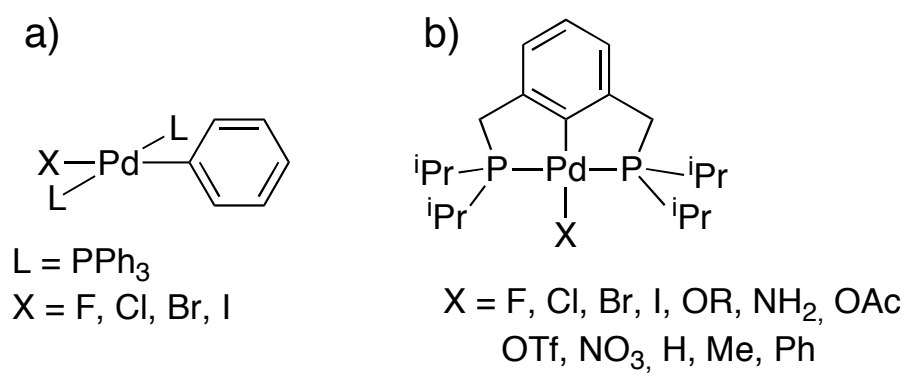

Figure 13. Palladium fluoro complexes studied by Grushin et al. (a, ref. 297) and Cámpora et al. (b, ref. 298).

$\pi$ Effects on the Pd-F bond have also been evaluated and the results are less intuitive than those obtained for $\sigma$-donation. The analysis of the structural and spectroscopic data of $\left[\mathrm{PdPhX}\left(\mathrm{PPh}_{3}\right)_{2}\right]$, such as the $\mathrm{C}-\mathrm{C}_{\mathrm{ipso}}-\mathrm{C}$ angle of the phenyl group, seem to indicate that the " $\mathrm{PdX}\left(\mathrm{PPh}_{3}\right)_{2}$ " fragment is more electron donor towards the phenyl ring when $\mathrm{X}=\mathrm{F} \cdot{ }^{297}$ In general, the meta position of a phenyl group is almost 
insensitive to $\pi$-effects whereas the para position strongly reflects the presence of electron donating or electron withdrawing groups in the ring. According to this, Cámpora et al. analyzed the difference in coordination shift between the meta and para positions of the aryl ring in the pincer ligand of $\left[\left({ }^{\mathrm{iPr}} \mathrm{PCP}\right) \mathrm{PdX}\right]$ (Figure 13, b). The differences found upon changing $X$, although small, should give a measure of the $\pi$ back donation from the metal. When $\mathrm{X}=\mathrm{F}$ the difference $\Delta \delta \mathrm{C}_{\mathrm{para}}-\Delta \delta \mathrm{C}_{\text {meta }}$ is one of the most negative, comparable to $\mathrm{X}=\mathrm{OR}$ or $\mathrm{NH}_{2}$, indicating that the metal behaves as a better electron donor to the aryl ligand. ${ }^{298}$ Since the empty orbital of palladium is not available for $\pi$-bonding with the fluoride and conjugation effects are very small, ${ }^{299}$ the observed effect has been interpreted as a result of a filled-filled $d \pi(P d)-p \pi(F)$ repulsion that drives the electron density on the metal towards the trans phenyl group.

Both effects, weak $\sigma$-donation and the proposed $\mathrm{d} \pi(\mathrm{Pd})-\mathrm{p} \pi(\mathrm{F})$ repulsion, leads to a polarized Pd-F bond and makes it more reactive than other Pd-halogen bonds. For example, when a chloride salt of a non coordinated anion is added to a solution of $\left[\mathrm{PdPhF}\left(\mathrm{PPh}_{3}\right)_{2}\right]$ in dichloromethane or chloroform, the fluoride dissociates from the complex and the so called "naked fluoride" is very reactive resulting in fluorination of $\mathrm{CH}_{2} \mathrm{Cl}_{2}$ or deprotonation of $\mathrm{CHCl}_{3}$ to give $\mathrm{HF}_{2}{ }^{-300}$ The presence of very small amounts of water in a solution of $\left[\mathrm{PdPhF}\left(\mathrm{PPh}_{3}\right)_{2}\right]$ leads to a fluxional process that transforms the $\mathrm{Pd}-\mathrm{F}{ }^{19} \mathrm{~F}$ NMR resonance from a sharp triplet to a broad singlet; the same effect is observed for the doublet in the ${ }^{31} \mathrm{P}$ NMR spectrum. The elimination of the ${ }^{2} \mathrm{~J}_{\mathrm{F}-\mathrm{P}}$ coupling constant can be the result of a fast ligand substitution of fluorine by water along with hydrogen bond formation with water Pd-F...HOH. ${ }^{290}$ This fluxional behaviour in the presence of water is quite general in $\mathrm{Pd}(\mathrm{II})$ fluoro derivatives.

The coordinated fluoride is basic enough to form hydrogen bonds both intra and intermolecularly. ${ }^{294}$ Pd-F...H-C interactions with the other coordinated ligands have 
been observed in monomeric $\left[\mathrm{PdPhF}\left(\mathrm{PR}_{3}\right)_{2}\right]$ compounds. When $\mathrm{R}=$ alkyl, the decomposition of the complexes leads, among other expected products, to $\mathrm{FHF}^{-}$and the source of $\mathrm{H}$ has been shown to be the alkyl groups of the phosphine. Thus, the preformed $\mathrm{Pd}-\mathrm{F} \ldots \mathrm{H}-\mathrm{C}$ interactions may have an influence in this process and therefore implications in the stability of the complexes in catalysis. Even a bridging fluoride is basic enough to form hydrogen bonds and a nice and unusual example has been described by Grushin et al. ${ }^{294}$ The dimeric complex $\left[\mathrm{Pd}(\mu-\mathrm{F}) \mathrm{Ph}\left(\mathrm{PC} y_{3}\right)\right]_{2}$ crystallizes with three molecules of dichloromethane that are hydrogen bonded to the bridging fluorides and the interaction is also kept in solution as shown by NMR (Figure 14).

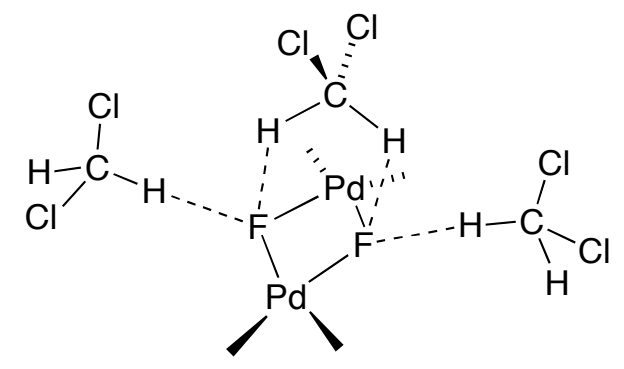

Figure 14. Simplified structure of the $\left[\mathrm{Pd}(\mu-\mathrm{F}) \mathrm{Ph}\left(\mathrm{PCy}_{3}\right)\right]_{2}$ core, showing the hydrogen bond interactions with $\mathrm{CH}_{2} \mathrm{Cl}_{2}$.

The palladium hydrogendifluoride $\left[\mathrm{PdPh}(\mathrm{FHF})\left(\mathrm{PR}_{3}\right)_{2}\right]$ has been thoroughly studied and the presence of the strong hydrogen bond to HF in the Pd-F...HF moiety leads to a Pd-F where the repulsive $\mathrm{d} \pi(\mathrm{Pd})-\mathrm{p} \pi(\mathrm{F})$ interaction is less important than for other palladium fluorides. The complex shows a fluxional behaviour as a result of a fast intramolecular fluoro exchange presumably through a pentacoordinated intermediate. ${ }^{301}$ Pd-F bonds can act not only as hydrogen bond acceptors but also as halogen bond acceptors. Perutz et al. have studied the interaction of complexes $\left[\mathrm{PdArF}\left(\mathrm{PR}_{3}\right)_{2}\right](\mathrm{Ar}=$ 2,3,5,6-tetrafluoropyridyl; $\mathrm{R}=\mathrm{Cy}$ ) and their $\mathrm{Ni}$ and $\mathrm{Pt}$ analogues with $\mathrm{C}_{6} \mathrm{~F}_{5} \mathrm{I}$. The M-F ${ }^{19} \mathrm{~F}$ NMR resonance is very sensitive to the interaction with the perfluorophenyl iodide (up to $20 \mathrm{ppm}$ ) and the thermodynamic parameters were determined by titration 
experiments using this technique. $\Delta \mathrm{H}$ for the interaction $\mathrm{Pd}-\mathrm{F} . . \mathrm{IC}_{6} \mathrm{~F}_{5}$ is $-21.7 \mathrm{~kJ} \mathrm{~mol}^{-1}$ with $-\Delta \mathrm{H}$ increasing in the order $\mathrm{Ni}<\mathrm{Pd}<\mathrm{Pt}$.

$\operatorname{Pd}(\mathrm{IV})$ fluoro complexes have also been reported in the context of the study of reductive elimination reactions of RF in high valent $\mathrm{Pd}$ species (see below). Monofluoro complexes have been detected, ${ }^{272,302}$ but most isolated and stable Pd(IV) compounds are di- or trifluoro derivatives. ${ }^{302,303}$

\subsection{Oxidative Addition of R-F.}

The cleavage of a C-F bond by oxidative addition of $\mathrm{R}-\mathrm{F}$ to a $\mathrm{Pd}(0)$ complex is a step generally involved in many catalytic reactions where C-F activation occurs and, in some cases, it has been shown to be the rate determining step of the reaction. ${ }^{236}$ The thermodynamics of the C-F activation by a metal complex may not be as unfavorable as one may think just looking at the C-F bond energy. Although this is quite a strong interaction the energy balance, that includes the formation of a M-F bond, may be more favorable for C-F cleavage than for other $\mathrm{C}-\mathrm{X}$ activation processes $(\mathrm{C}-\mathrm{H}$ for example). This has been shown by quantum calculations on Os and $\mathrm{Rh}$ complexes, where the reluctance to $\mathrm{C}-\mathrm{F}$ activation has a kinetic origin. ${ }^{304}$ In any case, the thermodynamics can be made more favorable by controlling the fate of the F atom after cleavage and providing an additional reaction acting as a thermodynamic sink. ${ }^{224}$ Thus, LiI has been used as additive to ease R-F activation by forming a $\left[\mathrm{PdRIL}_{2}\right]$ and $\mathrm{LiF}$ which has a larger lattice energy than LiI. ${ }^{245,246}$ Also, a the addition of a hard Lewis acid which can form an adduct with fluoride has the same effect. ${ }^{246}$

Oxidative addition of arylfluorides is preceded by arene coordination and this has been shown experimentally for $\mathrm{Ni}(0)$ compounds which coordinate 
octafluoronaphthalene, ${ }^{305}$ and fluorobenzenes. ${ }^{306,307}$ No analogous complexes have been detected or isolated for Pd and, although they are plausible intermediates. A reported calculation made on the model system with $\mathrm{L}=\mathrm{PH}_{3}$ could not locate them. ${ }^{308}$

The reaction of $\left[\mathrm{Pd}\left(\mathrm{PCy}_{3}\right)_{2}\right]$ with hexafluorobenzene leads to the expected oxidative addition product $\left[\mathrm{PdF}\left(\mathrm{C}_{6} \mathrm{~F}_{5}\right)\left(\mathrm{PCy}_{3}\right)_{2}\right] .{ }^{309}$ It is interesting to compare this behaviour to the one shown by the other members of group 10. The reaction of isolated or in situ generated $\left[\mathrm{NiL}_{2}\right]$ complexes $\left(\mathrm{L}=\mathrm{PR}_{3}, \mathrm{NHC}\right)$ leads to the same straightforward oxidative addition product as observed for palladium. ${ }^{307,310}$ However, when $\left[\mathrm{Pt}\left(\mathrm{PCy}_{3}\right)_{2}\right]$ reacts with pentafluorobenzene it leads to the formation of $\left[\mathrm{Pt}\left(\mathrm{C}_{6} \mathrm{~F}_{5}\right) \mathrm{Cy}\left(\mathrm{PCy}_{3}\right)\left(\mathrm{PFCy}_{2}\right)\right]$ where a new P-F bond has been formed and a cyclohexyl group is transferred from the phosphorous to the metal (Scheme 102). The reaction rate follows the order $\mathrm{Pt}>\mathrm{Pd}$, whereas the opposite $(\mathrm{Pd}>\mathrm{Pt})$ is observed in oxidative addition reactions with other non-fluorinated aryl halides. ${ }^{309}$ The same differences have been observed in the reactions of $\mathrm{M}(0)$ compounds $(\mathrm{M}=\mathrm{Ni}, \mathrm{Pd}, \mathrm{Pt})$ and pentafluoropyridine..$^{311,312}$ The most plausible mechanism for both $\mathrm{Ni}$ and $\mathrm{Pd}$ is the commonly accepted three-centered concerted addition, whereas a different pathway, a P-assisted C-F activation through a four-centered transition state, is operating for Pt (Scheme 102). ${ }^{313}$ The formation of this metalaphosphorane species opens up a new pathway for C-F cleavage and, although the formation of fluorinated phosphines has not been observed in the reactions of fluoroaryls with palladium( 0 ) complexes, the assistance of the P-F interactions in the CF cleavage cannot be neglected. As it will be mentioned later, P-F formation is relevant in the decomposition of palladium (II) fluoro complexes. 


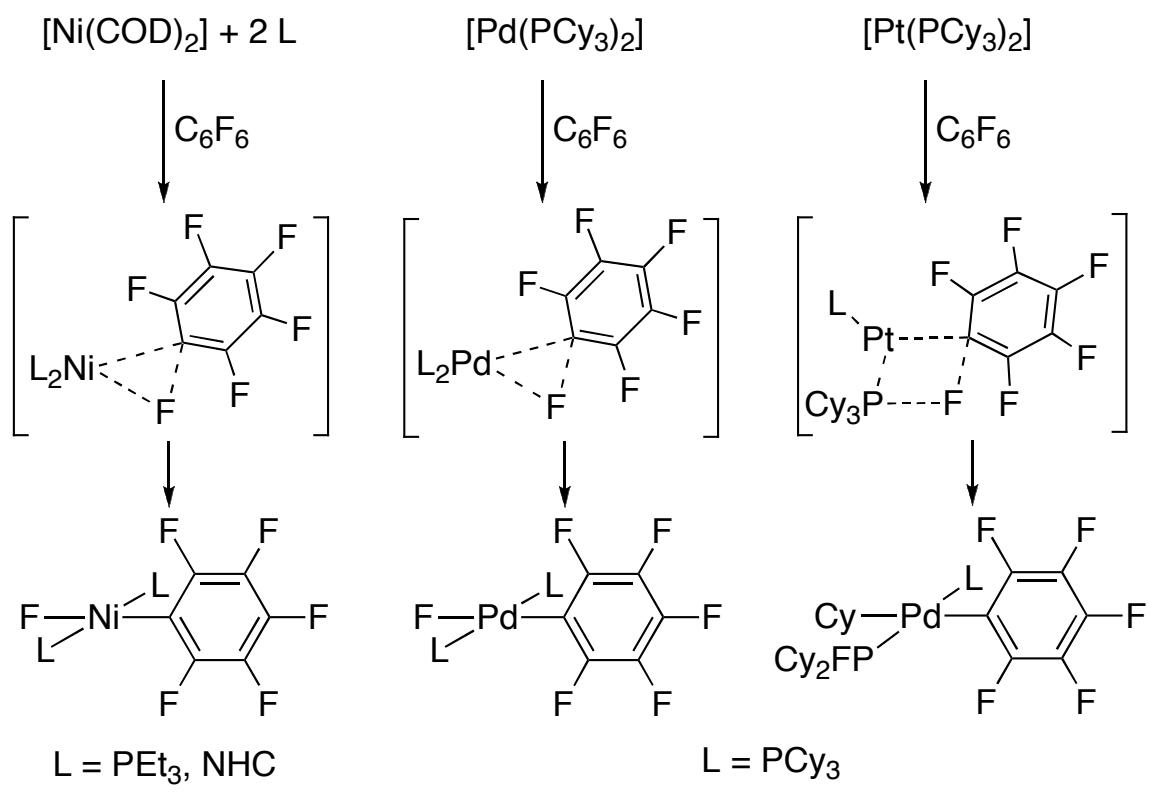

Scheme 102

Electron withdrawing groups on the fluoroaryl are generally needed in Pdcatalyzed reactions where C-F activation occurs. A computational study on the oxidative addition of monofluorobenzenes with different substituents has determined that the presence of electron withdrawing groups in the ring such as $\mathrm{NO}_{2}$ or $\mathrm{CN}$ lower the activation energy for a concerted three-membered transition state pathway. ${ }^{314}$ For activated electron poor aryls, an $\mathrm{S}_{\mathrm{N}} \mathrm{Ar}$ mechanism with the $\operatorname{Pd}(0)$ acting as the nucleophile can be proposed. These calculations indicate that this non-concerted pathway is possible and favored in polar solvents but the activation energy of both mechanism is not very different. The presence of an ortho nitro substituent produces the highest stabilization of the transition state for the oxidative addition by interaction with the Pd-center, as shown in a simplified representation in Figure 15. This effect is not found for an ortho $\mathrm{CN}$ group since its linear geometry prevents the coordination of the nitrogen to the metal. Further calculations on both the nitro and carboxyl systems show again that the coordination of an oxygen atom to the palladium in the transition state, considerably lowers the activation energy for the oxidative addition. ${ }^{234}$ The combination 
of either of these ortho groups and the presence of electron withdrawing substituents leads to the lowest barriers. Actually, the most successful C-F activation processes in catalytic C-C coupling reactions use fluoroaryls bearing ortho nitro, carboxyl or other coordinating (directing) groups as well as electron withdrawing groups on the ring (see above, section 3.1.1).

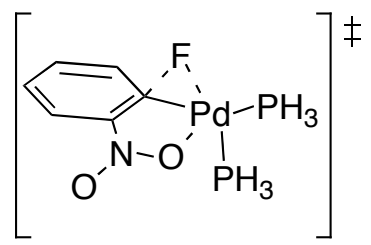

Figure 15. Simplified representation of the transition state for the oxidative addition of an ortho-nitro fluoroarene.

Oxidative addition products have been isolated for the reaction of $\operatorname{Pd}(0)$ complexes with $\mathrm{RF}$ where $\mathrm{R} \neq$ aryl. Upon thermolysis of a tetrafluoroethylene $\operatorname{Pd}(0)$ complex, a fluorovinyl palladium fluoride was isolated (Scheme 103). ${ }^{247}$ Doyle reported the oxidative addition of $\mathrm{HCOF}$ to $\left[\mathrm{Pd}\left(\mathrm{PPh}_{3}\right)_{4}\right]$. No formyl complex was formed and it appears that the product of the reaction is a hydrido fluoro complex resulting from oxidative addition of the formyl fluoride and subsequent decarbonylation to give the hydride. Although the characterization of the palladium hydride is poor, the analogous reaction with platinum, where a metal hydride was formed, supports the described pathway. $^{315}$

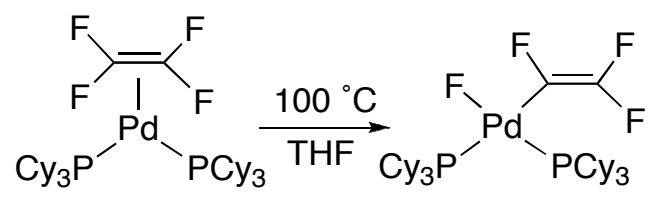

Scheme 103 
The allylic substitution reactions carried out with disubstituted cyclohexenyl fluorides and malonates by Hazari, Gouverneur and Brown showed that the oxidative addition of allyl fluorides to $\operatorname{Pd}(0)$ complexes is not as stereoselective as it is found for other analogous allylic halides or carboxylates. ${ }^{252}$ Generally, if a polar enough solvent is used, the oxidative addition of allylic derivatives is an $\mathrm{S}_{\mathrm{N}} 2$ process and inversion of configuration is observed. ${ }^{316}$ If a nucleophilic external attack follows, the overall stereochemistry expected in an allylic substitution process is retention of configuration. This is not the case of the allylic fluorides shown in Scheme $104 \mathrm{a}$, where retention is not observed and the same diastereomeric mixture is obtained regardless the configuration of the starting mixture. To explain this fact, the authors propose that after oxidative addition a tight ion pair between the cationic palladium allyl complex and fluoride is formed (Scheme 104,b). In this intermediate the external attack by $\operatorname{Pd}(0)$ species is faster than the malonate attack and produces the inversion of the configuration of the allylic palladium derivative prior to the formation of the final product. This inversion process has already been studied for other palladium allyls. ${ }^{317}$ In this work it was also found that the rate of oxidative addition of allylX follows the trend $\mathrm{X}: \mathrm{OCO}_{2} \mathrm{Me}>\mathrm{OCOPh}>>\mathrm{F}>>\mathrm{OCOMe}$. 
a)

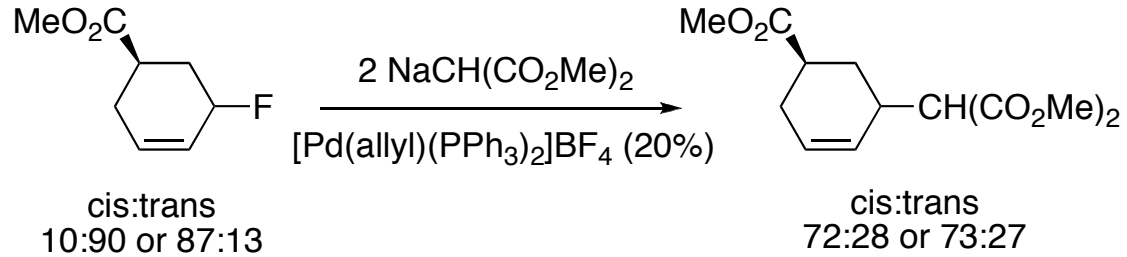

b)

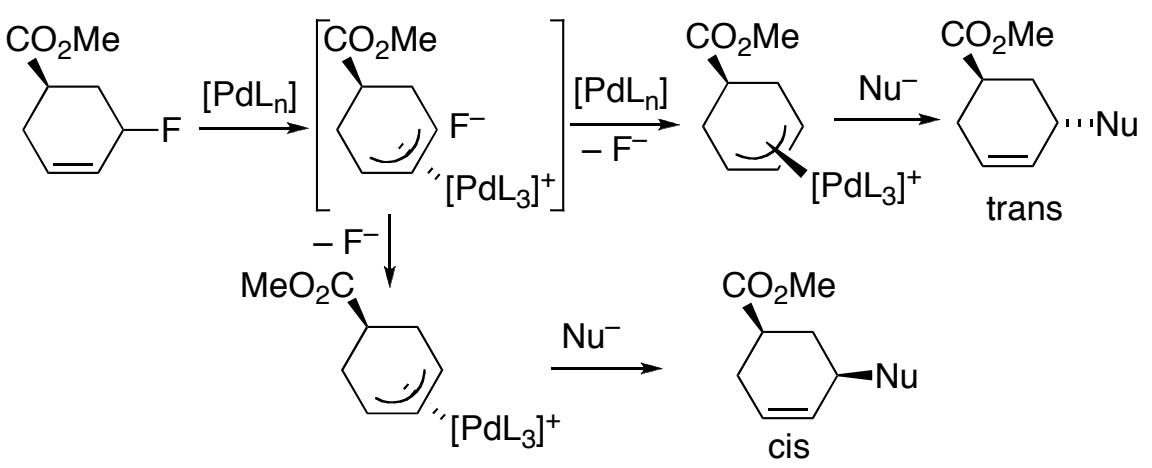

Scheme 104

\section{4. $\beta-F$ and $\alpha-F$ elimination.}

Another route for the cleavage of $\mathrm{C}-\mathrm{F}$ bonds is $\beta-\mathrm{F}$ elimination in a preformed palladium alkyl, which occurs in some of the catalytic processes described above. This process has been compared to other $\beta$-X elimination reactions by computational methods including the most common $\beta$-H elimination. ${ }^{318}$ When $\mathrm{X}=\mathrm{Cl}, \mathrm{Br}$, I it has been found that $\beta$-halogen elimination is kinetic- and thermodynamically more favored than $\beta-\mathrm{H}$ elimination, and this is generally observed experimentally. $\beta$-F elimination is the least favored of all $\beta$-halogen elimination reactions for both kinetic and thermodynamic reasons since the differences in dissociation energies between the $\mathrm{C}-\mathrm{X}$ and $\mathrm{Pd}-\mathrm{X}$ bonds is the least favorable for $\mathrm{X}=\mathrm{F}$. When compared to $\mathrm{X}=\mathrm{H}, \beta-\mathrm{H}$ elimination is kinetically more favorable that $\beta-\mathrm{F}$ elimination but the final $\operatorname{Pd}($ alkene) $\mathrm{X}$ complex is less stable when $\mathrm{X}=\mathrm{H}$ and therefore the overall reaction is less favored thermodynamically. Nonetheless, being faster, $\beta-\mathrm{H}$ elimination is likely to occur 
whenever this step is combined with an additional reaction that provides the driving force to make the whole process exothermic. In fact, the experimental examples where $\beta-\mathrm{F}$ elimination are reported occur on derivatives where $\beta-\mathrm{H}$ elimination is not possible. ${ }^{248-251}$

The reactivity of $\sigma$-perfluoroalkyl metal complexes is largely dominated by $\alpha$ fluorine elimination processes, which lead to the formation of fluorocarbene derivatives (Scheme 105).

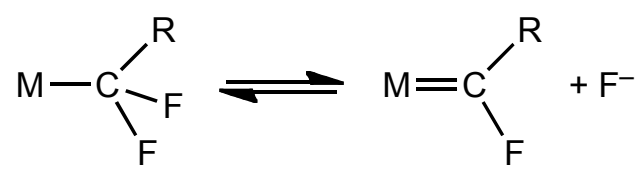

Scheme 105

This is a well-known process and most of its basis as well as the reactivity of the resulting fluorocarbene derivatives were explained and compiled in an early revision by Brothers and Roper. ${ }^{319}$ The reactivity of transition metal perfluoroalkyls via $\alpha$-fluorine activation has been recently reviewed. ${ }^{320}$ Since transition metal fluorocarbenes are highly reactive species they often react before being isolated. In palladium chemistry there are no reports on stable, isolated fluorocarbenes derived of alpha-fluorine elimination in fluoroalkyl palladium compounds. However, the $\alpha$-elimination reaction seems to provide a low energy decomposition pathway that can be reached under the conditions in which some catalytic reactions take place. This effect has been reported by Grushin and Marshall on their study of the decomposition of $\left[\mathrm{Pd}\left(\mathrm{CF}_{3}\right) \mathrm{Ph}(\mathrm{dppe})\right] .{ }^{163} \mathrm{The}$ first step of this reaction is the reduction of a catalytic amount of the starting palladium(II) complex to palladium(0). The authors proposed several competitive pathways for this reduction, one of them the activation of the $\mathrm{CF}_{3}$ by $\alpha$-fluorineelimination, followed by hydrolysis of the resulting difluorocarbene. The hydrolysis of 
difluorocarbenes is a well-known process that leads to the formation of a carbonyl ligand. $^{319,320}$ The insertion of the CO ligand into the Pd-Ar bond and its subsequent elimination with the remaining fluoride produces benzoyl fluoride, one the observed species in this reaction. Supporting this interpretation, several of the expected decomposition products have been found in the reaction media. ${ }^{289}$ Other decomposition products proceed from the hydrolytic cleavage of the fluoride in the same intermediate (Scheme 106).

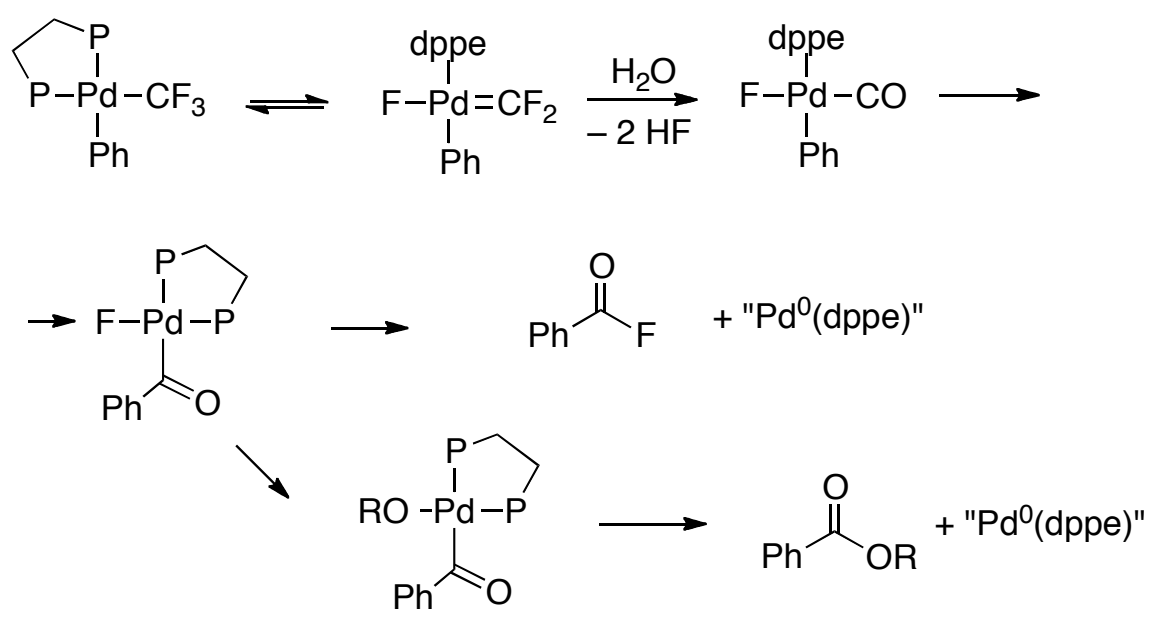

$(\mathrm{R}=\mathrm{H}, \mathrm{COC} 6 \mathrm{H} 5)$

Scheme 106

\subsection{Other activation routes for $\mathrm{C}-\mathrm{F}$ cleavage.}

Metal mediated examples nucleophilic attack on the C-F bond by different electron rich groups have been reported. ${ }^{224}$ Although its relevance in metal catalyzed processes with $\mathrm{C}-\mathrm{F}$ activation is lower that the processes described above, interesting stoichiometric reaction have been described where the presence of the metal may have a role in bringing the fluorinated group and the nucleophile close to each other, reducing the activation entropy of the process and directing the regiochemistry of the attack. 
For example, the reaction of the tetranuclear imidoyl bridged complex in Scheme 107 with $\mathrm{PPh}_{2} \mathrm{H}$ in a non polar solvent simply gives the $\mathrm{Cl}$ bridge splitting reaction. However, in a polar solvent and in the presence of $\mathrm{KOH}$ to promote the formation of diphenylphosphide, the nucleophilic substitution of a $\mathrm{F}_{\text {ortho }}$ by $\mathrm{PPh}_{2}$ is produced. The new complex was easily identified by the observation of only four $\mathrm{F}$ resonances $(1: 1: 1: 1)$ and was confirmed by X-ray diffraction. ${ }^{321}$

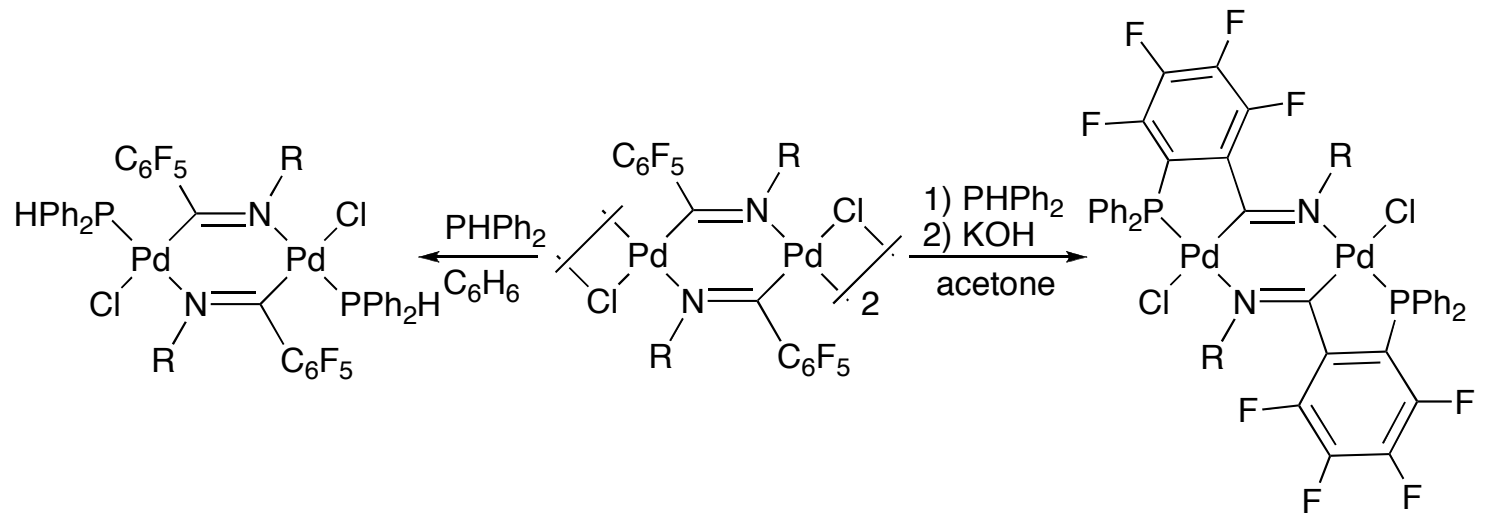

Scheme 107

\subsection{Reductive elimination of R-F}

The reductive elimination of aryl fluorides in $\mathrm{Pd}(\mathrm{II})$ complexes [PdArFL 2 is not an easy and straightforward process. This may be the main reason for the scarce efficient $\mathrm{ArF}$ forming reactions based on a $\mathrm{Pd}(0) / \mathrm{Pd}(\mathrm{II})$ mechanism that have been reported (see above), since reductive elimination is the last step in the catalytic cycle.

Grushin and coworkers have devoted a lot of effort to study the ArF reductive elimination reaction on isolated palladium fluoro complexes. A nice account summarizing this work has been written. ${ }^{227}$ The decomposition of palladium(II) fluoro derivatives $[\mathrm{PdFArL} 2]\left(\mathrm{L}=\mathrm{PR}_{3}\right)$ does not yield ArF but products derived from other competitive reactions, mainly P-F formation and aryl exchange. This occurs for both 
monodentate, ${ }^{322}$ and also bidentate phosphines. ${ }^{192,323}$ Scheme 108 collects the products observed for $\mathrm{L}=\mathrm{PPh}_{3}$ and the plausible mechanistic pathway that explains the products observed and fits the experimental data. ${ }^{295,322}$ As can be seen in Scheme 108, the P-F forming process is intramolecular and it does not depend on the concentration of $\mathrm{PR}_{3}$. This is important since its occurrence cannot be controlled by changing the concentration of free phosphine in a catalytic process rendering most phosphine ligands useless in ArF forming catalysis.

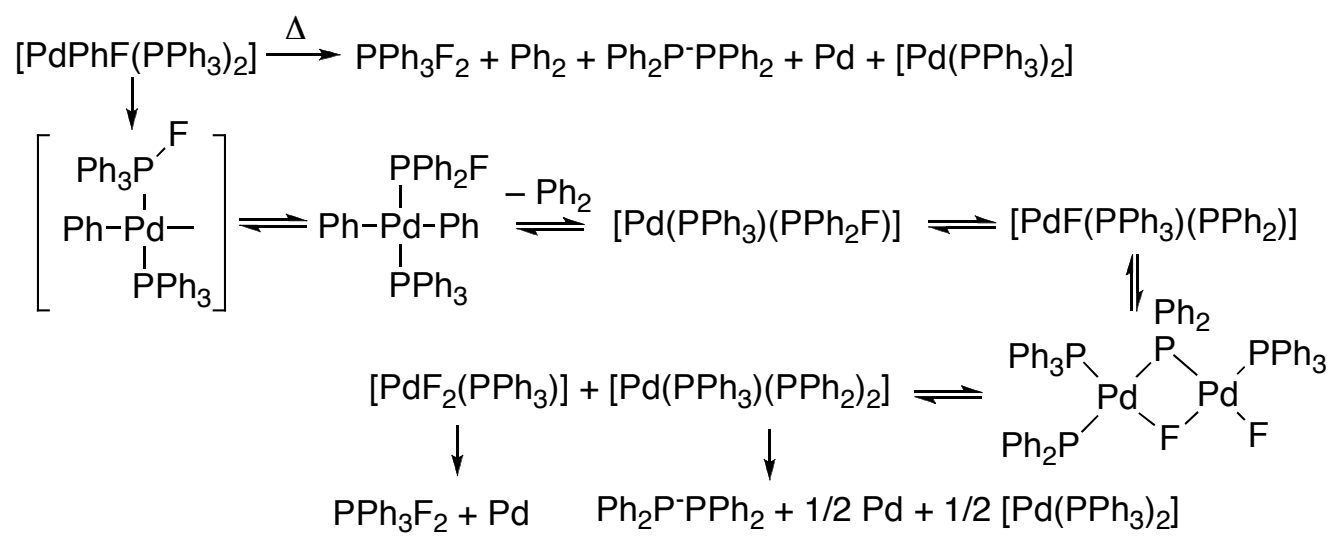

Scheme 108

The most successful ligands for reductive elimination of $\operatorname{ArF}$ in $\operatorname{Pd}(\mathrm{II})$ complexes are Buchwald's BrettPhos derivatives (Scheme 109). These bulky monodentate phosphines form monomeric [PdArFL] complexes where a formally tricoordinated $\mathrm{Pd}(\mathrm{II})$ center is present, although it is actually a four-coordinated $\mathrm{Pd}(\mathrm{II})$ with a weakly bound $\mathrm{C}_{\mathrm{ipso}}$ of one of the phosphine aryl rings. DFT calculations have shown that reductive elimination of $\mathrm{ArF}$ is feasible in three coordinated $\mathrm{Pd}(\mathrm{II})$ complexes and the activation energy is compatible with synthetic applications. ${ }^{296}$ Since [PdArFL] complexes $(\mathrm{L}=$ Brettphos) easily reach a low coordination number and these bulky ligands prevent the formation of dimeric complexes, they are suitable to give a kinetically favorable ArF elimination. Indeed, Buchwald et al. have demonstrated that 
thermolysis of $[\mathrm{PdArFL}]$ complexes $(\mathrm{L}=$ BrettPhos) is possible leading to $\mathrm{ArF}$ and $\operatorname{Pd}(0)$ which can be trapped by addition of an aryl bromide, which oxidatively adds to the $\operatorname{Pd}(0)$ byproduct. $^{279,282}$ Aryl groups with electron withdrawing substituents lead to easier ArF elimination, ${ }^{279}$ but a right choice of ligand allows the formation of $\mathrm{ArF}$ even for aryl groups with electron donating substituents albeit in lower yields (Scheme 109). ${ }^{282}$ There has been some discussion about the actual mechanism or ArF formation when the aryl group bears electron withdrawing groups, specially $\mathrm{NO}_{2}$, since in this case a classical $S_{N} A r$ substitution can be invoked instead of a concerted reductive elimination. ${ }^{295}$ However, the examples reported for the BrettPhos type ligands seem to be general enough to be considered as actual reductive eliminations from $\mathrm{Pd}(\mathrm{II})$.

$$
[\mathrm{PdArFL}] \longrightarrow \text { ArF }+[\mathrm{PdL}] \stackrel{\mathrm{ArBr}}{\longrightarrow}[\mathrm{PdArBrL}]
$$

a) Up to $50 \%$
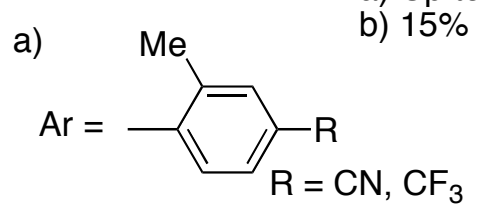

b)<smiles>[14C]c1ccc(Br)cc1</smiles><smiles>COc1ccc(OC)c(P(=O)(O)OC)c1-c1c(P)cc(C(C)C)cc1C(C)C</smiles>

$L=$ BrettPhos<smiles>CCCCc1c(OC)ccc(OC)c1-c1c(C(C)C)cc(C(C)C)c(-c2ccc(Br)cc2)c1C(C)C</smiles>

$\mathrm{L}={ }^{\mathrm{t}} \mathrm{BuBrettPhos}-\mathrm{Ar}$

Scheme 109

The reductive elimination from a palladium(IV) fluoro complex has been proposed in many fluorination reactions catalyzed by electrophilic fluorinating reagents. Ritter et al. demonstrated that this is a plausible pathway by synthesizing fluoro quinolinato $\mathrm{Pd}(\mathrm{IV})$ complexes that cleanly decompose at $50{ }^{\circ} \mathrm{C}$ in solution to give the fluorinated quinolinato ligand and a $\mathrm{Pd}(\mathrm{II})$ complex. $^{272,302}$ They have studied the 
mechanism of decomposition of these complexes in detail and found out that decoordination of one of the ligands is the most plausible pathway, followed by a rate determining reductive elimination of $\mathrm{ArF}$ in a pentacoordinated intermediate (Scheme 110). ${ }^{272}$ Electron withdrawing substituents on the aryl group that generates the fluoroaryl $\left(\mathrm{R}^{3}\right.$, Scheme 110) favor the reaction, showing that the reductive elimination occurs by interaction of an electrophilic carbon center and a nucleophilic fluoride. The influence of substituents with different electronic properties on the auxiliary ligands $\left(\mathrm{R}^{1}, \mathrm{R}^{2}\right.$, Scheme 110) led to propose the depicted transition state for reductive elimination. The authors argue that the ancillary ligand they use, a pyridyl sufonamide, is perfectly suited for this type of reaction being able to act as a tridentate ligand for $\operatorname{Pd}(\mathrm{IV})$ and a bidentate one in the resulting $\mathrm{Pd}(\mathrm{II})$ complex. A close related $\mathrm{Pd}(\mathrm{IV})$ difluoro complex $\left[\mathrm{PdF}_{2}\left(\mathrm{~N}-\mathrm{NSO}_{2} \mathrm{R}\right)(\right.$ benzoquinolinato $\left.)\right]$ was also isolated and characterized. Upon thermolysis, it is more stable than the monofluoro palladium complexes and it decomposes through reductive elimination of $\mathrm{RF}(\mathrm{R}=$ benzoquinolinato) at higher temperatures $\left(150{ }^{\circ} \mathrm{C}\right.$ in DMSO). The mechanism of the reaction is complicated and competition with reductive elimination of $F_{2}$ has also been observed.

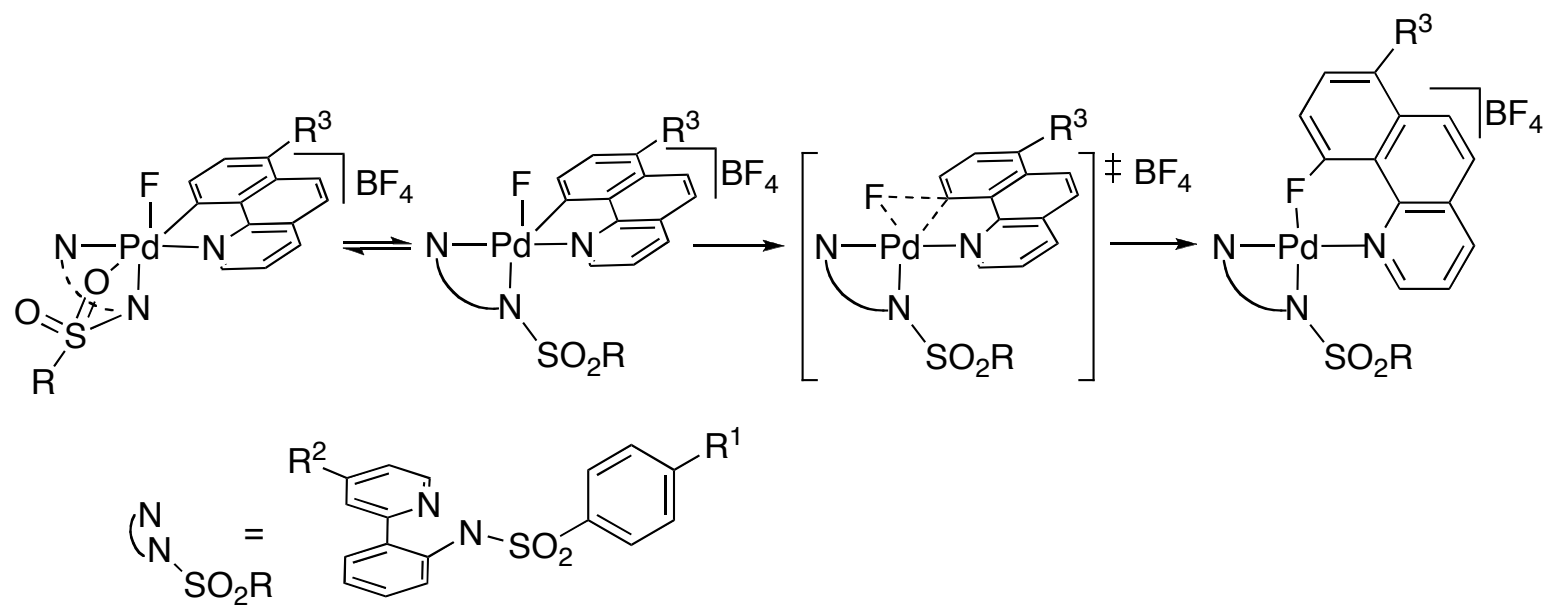


The reluctance of a trifluoro aryl Pd(IV) complex to give ArF by reductive elimination was observed by Ball and Sanford. $^{303}$ In this case the difluorohydrogendifluoride complex does not lead to reductive elimination upon heating but to significant amounts of biaryl formation. The excess of an oxidant promotes the reductive elimination of the aryl fluoride, but the mechanism has not been ascertained (Scheme 111).

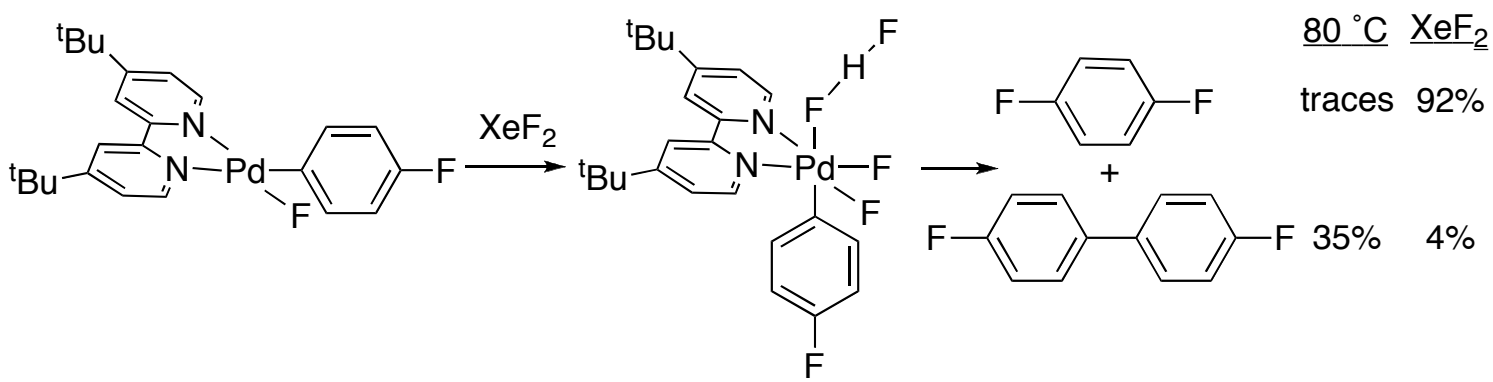
Scheme 111

Recently, Sanford's group reported the reductive elimination reaction from $\mathrm{Pd}(\mathrm{IV})$ complexes containing both $\mathrm{Pd}-\mathrm{C}\left(\mathrm{sp}^{2}\right)$ and $\mathrm{Pd}-\mathrm{C}\left(\mathrm{sp}^{3}\right)$ bonds (Scheme 112). Only the formation of a $\mathrm{C}\left(\mathrm{sp}^{3}\right)-\mathrm{F}$ bond is observed. ${ }^{324}$ The kinetics of the reductive elimination reaction were studied for $\mathrm{L}=\mathrm{py}$, finding an inverse first-order dependence on the concentration of pyridine, that implies the dissociation of the pyridine ligand prior to the rate-determining step. According to this, it is proposed that the elimination takes place from a cationic pentacoordinated intermediate. DFT calculations show that the barrier for the reductive elimination of the $\mathrm{C}\left(\mathrm{sp}^{2}\right)$ fragment has an energy that exceeds in 5-12 $\mathrm{kcal} \mathrm{mol}{ }^{-1}$ the calculated energy for the $\mathrm{C}\left(\mathrm{sp}^{3}\right)$ elimination (the exact energy value depends on the geometry considered for the pentacoordinate intermediate). 

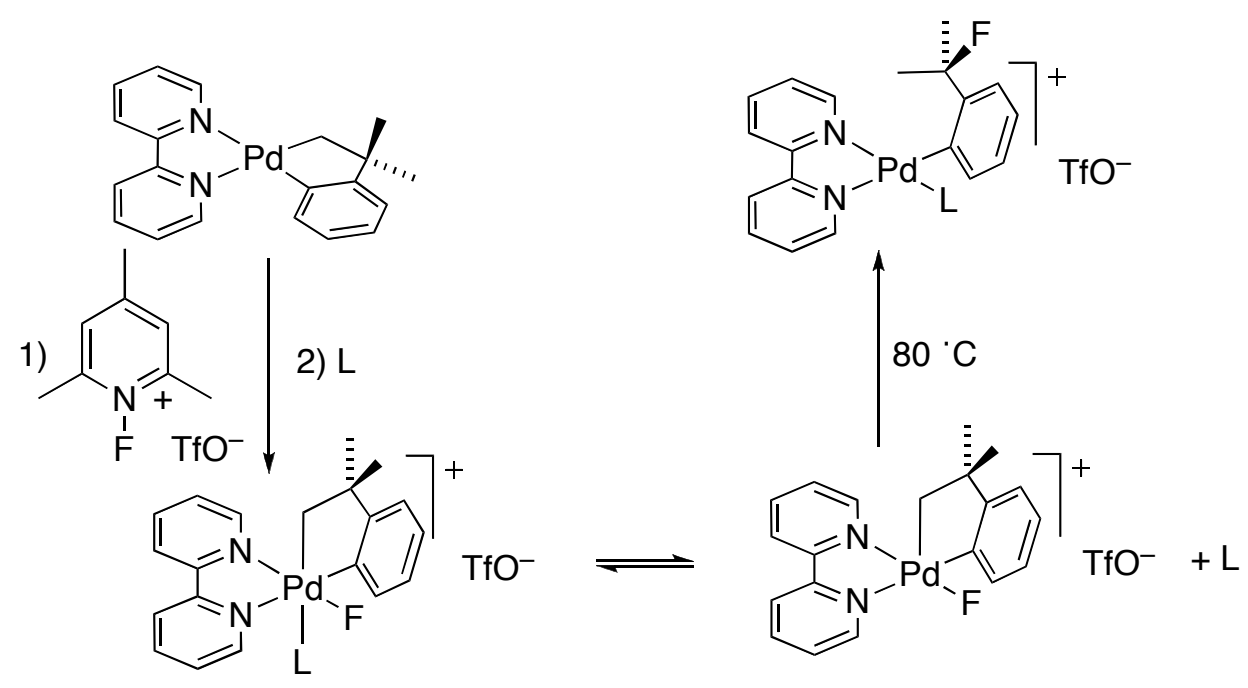

Scheme 112

In the same type of complexes, Pérez Temprano et al. have studied the competitive formation of $\mathrm{C}-\mathrm{F}$ bonds versus $\mathrm{C}-\mathrm{N}$ bonds in the reductive elimination from Pd(IV) complexes (Scheme 113). ${ }^{325}$ By heating in acetonitrile the mixture of isomers shown in Scheme 113, three of the possible products of the reductive elimination process are obtained. Significantly, no elimination of the aryl $\mathrm{Csp}^{2}$ carbon is observed, neither to form a $\mathrm{C}-\mathrm{F}$ bond nor a $\mathrm{C}-\mathrm{N}$ one. The reaction conditions can be changed to produce selectively and almost quantitatively the formation of the $\mathrm{C}-\mathrm{N}$ bond by adding 2 equivalents of bipyridine along with 1 equiv of $\mathrm{NMe}_{4} \mathrm{NHTs}$.
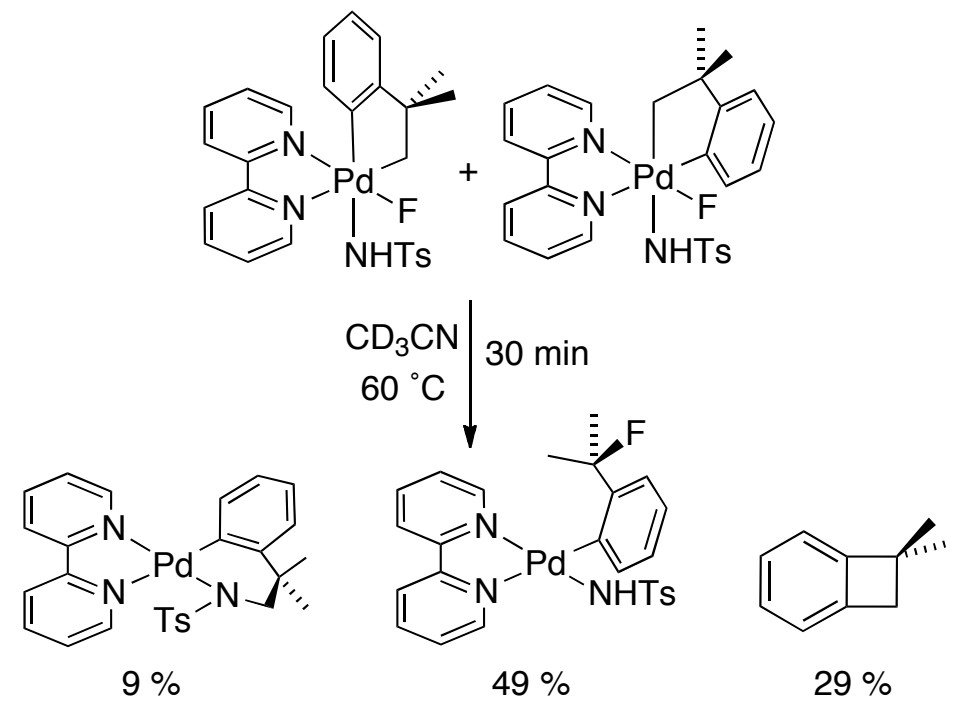

Scheme 113 


\section{Conclusion}

The previous pages show recent advances in the chemistry of palladium that improve its use as catalyst for the synthesis of organofluorinated compounds. In recent years there has been much progress in understanding the particularities of these systems and in how the fundamental reaction steps (reductive elimination, insertion and oxidative addition) are affected by the presence of fluorine atoms in the substrates. It is also remarkable the development in the use of palladium complexes in high oxidation states in catalysis and stoichiometric synthesis. Despite these advances, to gather the organometallic chemistry and catalysis of palladium complexes with fluorinated and non-fluorinated substrates in the same general scheme remains to be a difficult task. Hopefully, the great interest and ongoing research efforts in the area will lead to significant progress in the next few years.

\section{Acknowledgements.}

This work was supported by the Junta de Castilla y León (Grants no. VA302U13 and VA256U13).

\section{References}

${ }^{1}$ Kirsch P. Modern Fluoroorganic Chemistry. 2nd ed. Weinheim, Germany: Wiley-VCH; 2013.

${ }^{2}$ Purser S, Moore PR, Swallow S, Gouverneur V. Fluorine in medicinal chemistry. Chem. Soc. Rev. 2008;37:320-330.

${ }^{3}$ Liang T, Neumann CN, Ritter T. Introduction of fluorine and fluorine-containing functional groups. Angew. Chem. Int. Ed. 2013;52:8214-8264.

4 (a) Ma JA, Cahard D. Asymmetric fluorination, trifluoromethylation, and perfluoroalkylation reactions. Chem. Rev. 2008;108:PR1-PR43. (b) ibid Chem Rev. 2004;104:6119-6146. (c) Nie J, Guo HC, Cahard D, Ma JA. Asymmetric construction of stereogenic carbon centers 
featuring a trifluoromethyl group from prochiral trifluoromethylated substrates. Chem Rev. 2011;111:455-529.

${ }^{5}$ Ojima I. Exploration of fluorine chemistry at the multidisciplinary interface of chemistry and biology. J Org Chem. 2013;78:6358-6383.

${ }^{6}$ Torrens H. Carbon fluorine bond activation by platinum group metal complexes. Coord. Chem. Rev. 2005;249:1957-1985.

${ }^{7}$ Amii H, Uneyama K. C-F bond activation in organic synthesis. Chem. Rev. 2009;109:21192183.

${ }^{8}$ McClinton MA, McClinton, DA. Trifluoromethylations and related reactions in organic chemistry Tetrahedron. 1992;48:6555-6666.

${ }^{9}$ Umemoto T. Electrophilic perfluoroalkylating agents. Chem Rev. 1996;96:1757-1777

${ }^{10}$ Jin Z, Hammond GB, Xu B. Transition-metal-mediated fluorination, difluoromethylation, and trifluoromethylation. Aldrichim, Acta 2012;45:67-83.

${ }^{11}$ Zhang C-P, Chen Q-Y, Guo Y, Xiao Y-C, Gu Y-C. Difluoromethylation and trifluoromethylation reagents derived from tetrafluoroethane-sultone: Synthesis, reactivity and applications. Coord Chem Rev. 2014;261:28-72.

${ }^{12}$ Murphy PM, Baldwin CS, Buck RC. Syntheses utilizing n-perfluoroalkyl iodides [RFI, $\left.\mathrm{C}_{\mathrm{n}} \mathrm{F}_{2 \mathrm{n}+1} \mathrm{-I}\right]$ 2000-2010. J Fluorine Chem. 2012;138:3-23.

${ }^{13}$ Roy S, Gregg BT, Gribble GW, Le V-D, Roy S. Trifluoromethylation of aryl and heteroaryl halides. Tetrahedron 2011;67:2161-2195.

${ }^{14}$ Liu H, Gu Z, Jiang X. Direct trifluoromethylation of the C-H bond. Adv Synth Catal. 2013;355:617-626.

${ }^{15}$ Sato K, Tarui A, Omote M, Ando A, Kumadaki I. Trifluoromethylation of organic compounds and related reactions. Synthesis 2010;11:1865-1882.

${ }^{16}$ Prakash GKS, Hu J. Selective Fluoroalkylations with Fluorinated Sulfones, Sulfoxides, and Sulfides. Acc Chem Res. 2007;40:921-930.

${ }^{17} \mathrm{Xu}$ J, Liu X, Fu Y. Recent advance in transition-metal-mediated trifluoromethylation for the construction of C(sp3)-CF 3 bonds. Tetrahedron Lett. 2014;55:585-594.

${ }^{18} \mathrm{Ye} \mathrm{Y}$, Sanford MS. Investigations into transition-metal-catalyzed arene trifluoromethylation reactions. Synlett 2012;23:2005-2013.

${ }^{19}$ Lundgren RJ, Stradiotto M. Transition-metal-catalyzed trifluoromethylation of aryl halides. Angew Chem Int Ed. 2010;49:9322-9324.

${ }^{20}$ Liu T, Shen Q. Progress in Copper-Mediated Formation of Trifluoromethylated Arenes. Eur J Org Chem. 2012:6679-6687.

${ }^{21}$ (a) García-Monforte MA, Alonso PJ, Forniés J, Menjón B. Dalton Trans. 2007;3347-3359.

(b) Usón R, Forniés J, Tomás M. Anionic perhaloaryl-palladium and -platinum complexes as sources of unusual homo- and hetero-nuclear compounds. J. Organomet. Chem. 1988;358:525-543. (d) Usón R, Forniés J. Organopalladium and platinum compounds with perhalophenyl ligands. Adv. Organomet. Chem. 1988;28:219-297.

${ }^{22}$ García-Monforte MA, Martínez-Salvador S, Menjón B. The trifluoromethyl group in transition metal chemistry. Eur. J. Inorg. Chem. 2012;4945-4966.

${ }^{23}$ (a) Albéniz AC, Espinet P, López-Cimas O, Martín-Ruiz B. Dimeric palladium complexes with bridging aryl groups: When are they stable? Chem. Eur. J. 2005;11:242-252. (b) Usón R, Forniés J, Tomás M, Casas JM, Navarro R. Synthesis and reactivity of binuclear homometallic or hetero-metallic complexes $\left[\mathrm{NBu}_{4}\right]_{2}\left[\mathrm{MM}^{\prime}\left(\mu-\mathrm{C}_{6} \mathrm{~F}_{5}\right)_{2}\left(\mathrm{C}_{6} \mathrm{~F}_{5}\right)_{4}\right]\left(\mathrm{M}=\mathrm{M}^{\prime}=\mathrm{Pd}\right.$ or Pt, $\mathrm{M}$ $=\mathrm{Pt}, \mathrm{M}^{\prime}=\mathrm{Pd}$ ) with bridging pentafluorophenyl groups. J. Chem. Soc. Dalton Trans. 1989;169-172.

${ }^{24}$ Usón R, Forniés J, Falvello LR, et al. Synthesis and structure of two dinuclear anionic complexes, with $\mathrm{Pt}(\mathrm{III})-\mathrm{Pt}(\mathrm{III})$ bonds and unprecedented $\left(\mathrm{C}_{6} \mathrm{~F}_{4} \mathrm{O}\right)^{2-}$ or $\left\{\mathrm{C}_{6} \mathrm{~F}_{4}(\mathrm{OR})_{2}\right\}^{2-}(\mathrm{R}=\mathrm{Me}$, Et) quinone-like bridging ligands. J. Am. Chem. Soc. 1994;116:7160-7165.

${ }^{25}$ See for instance: For turnstile processes: (a) Casares JA, Espinet P. Dynamic behavior of $\left[\mathrm{Pd}\left(\mathrm{C}_{6} \mathrm{~F}_{5}\right)_{2}\left(\mathrm{SPPy}_{\mathrm{n}} \mathrm{Ph}_{3-\mathrm{n}}\right)\right]$ complexes: Evidence for a turnstile mechanism in intramolecular exchange. Inorg Chem. 1997;36:5428-5431. For isomerization reactions: (b) Casado AL, 
Casares JA, Espinet P. Mechanism of the uncatalyzed dissociative cis-trans isomerization of bis(pentafluorophenyl)bis(tetrahydrothiophene): A refinement. Inorg Chem. 1998;37:41544156. (c) Albéniz AC, Casado AL, Espinet P. Atropisomerization in cis- $\left[\mathrm{Pd}\left(2-\mathrm{C}_{6} \mathrm{BrF}_{4}\right)_{2} \mathrm{~L}_{2}\right]$ ( $\mathrm{L}=$ thioether): A dual mechanism involving ligand-dissociative and nondissociative competitive pathways. Inorg. Chem. 1999;38:2510-2515. For dissociative processes: (d) Casares JA, Coco S, Espinet P, Lin Y-S. Observation of a slow dissociative process in palladium(II) complexes. Organometallics, 1995;14:3058-3067. (e) Casares JA, Espinet P, Martínez-Ilarduya JM, Lin Y-S. Kinetic study of the dynamic behavior of $\left[\mathrm{M}\left(\mathrm{C}_{6} \mathrm{~F}_{5}\right) \mathrm{X}\left(\mathrm{OPPy}_{\mathrm{n}} \mathrm{Ph}_{3-\mathrm{n}}\right)\right]\left(\mathrm{M}=\mathrm{Pd}, \mathrm{Pt} ; \mathrm{X}=\mathrm{C}_{6} \mathrm{~F}_{5}\right.$, halide; $\left.\mathrm{n}=1,2,3\right)$ : Activation parameters for the restricted rotation about the $\mathrm{M}$-aryl bond, and for the Py associative exchange. Organometallics, 1997;16:770-779. (f) Albéniz AC, Casado AL, Espinet P.

Atropisomerization in cis- $\left[\mathrm{Pd}\left(2-\mathrm{C}_{6} \mathrm{BrF}_{4}\right)_{2} \mathrm{~L}_{2}\right](\mathrm{L}=$ Thioether $)$ : A dual mechanism involving ligand-dissociative and nondissociative competitive pathways. Inorg. Chem. 1999;38:25102515. For other fluxional processes: (g) Casares JA, Espinet P, Soulantica K, Pascual I, Orpen AG. $\mathrm{P}\left(\mathrm{CH}_{2} \mathrm{CH}_{2} \mathrm{Py}\right)_{n} \mathrm{Ph}_{3-\mathrm{n}}(\mathrm{Py}=2$-pyridyl; $\mathrm{n}=1,2,3)$ as chelating and as binucleating ligands towards palladium. Inorg Chem. 1997;36:5251-5256. (h) Alonso MA, Casares JA, Espinet P, Martínez-Ilarduya JM, Pérez-Briso C. The 3,5 dichlorotrifluorophenyl ligand, a useful tool for the study of coordination modes and dynamic behavior of complexes of palladium and platinum. Eur J Inorg Chem. 1998:1745-1753. (i) Carrión MC, Guerrero A, Jalón FA, et. al. J. Five different fluxional processes in polyfluorophenyl palladium(II) complexes with 2,4,6-tris(3,5-dimethylpyrazol-1-yl)-1,3,5-triazine. The driving effect of the solvent. Inorg Chem. 2003;42(3):885-895.

${ }^{26}$ Casares JA, Espinet P, Salas G. Palladium catalysts for fast norbornene polymerization. A study by NMR and calorimetric methods. Organometallics. 2008;27(15):3761-3769.

${ }^{27}$ Espinet P, Albéniz AC, Casares JA, Martínez-Ilarduya JM. ${ }^{19} \mathrm{~F}$ NMR in organometallic chemistry: Applications of fluorinated aryls. Coord Chem Rev. 2008;252:2180-2206.

${ }^{28}$ Högermeier J, Reissig HU. Nine times fluoride can be good for your syntheses. not just cheaper: nonafluorobutanesulfonates as intermediates for transition metal-catalyzed reactions. Adv. Synth. Catal. 2009;351:2747-2763.

${ }^{29}$ Lechel T, Dash J, Hommes P, Lentz D, Reissig HU. Three-component synthesis of perfluoroalkyl- or perfluoroaryl-substituted 4-hydroxypyridine derivatives and their palladium-catalyzed coupling reactions. J. Org. Chem. 2010;75:726-732.

${ }^{30}$ Pan Y, Ruhland B, Holmes CP. Use of a perfluoroalkylsulfonyl (PFS) linker in a "traceless" synthesis of biaryls through Suzuki cleavage. Angew. Chem. Int. Ed. 2001;40:4488-4491.

${ }^{31}$ Gerfaud T, Neuville L, Zhu J. Palladium-catalyzed annulation of acyloximes with arynes (or alkynes): Synthesis of phenanthridines and isoquinolines. Angew. Chem. Int. Ed. 2009;48:572-577.

${ }^{32}$ Chen M-W, Duan Y, Chen Q-A, Wang D-S, Yu C-B, Zhou Y-G. Enantioselective Pdcatalyzed hydrogenation of fluorinated imines: facile access to chiral fluorinated amines. Org Lett. 2010;12(21):2010.

${ }^{33}$ a) Huang YZ, Zhou QL. Studies on the Pd catalyzed reaction of perfluoroalkyl and polyfluoroalkyl iodides wits tertiary amines. Tetrahedron Lett. 1986;27:2397-2400. (b) Huang Y-Z, Zhou QL. Nickel-, palladium-, and platinum-catalyzed reactions of perfluoroand polyfluoroalkyl iodides with tertiary amines. J Org Chem. 1987;52:3552-3558.

${ }^{34}$ Ishihara T, Kuroboshi M, Okada Y. New efficient palladium-catalyzed perfluoroalkylation of carbon-carbon multiple bonds with F-alkyl iodides - An expedient route to F-alkylated alkyl and alkenyl iodides. Chem Letters. 1986;11:1895-1896.

${ }^{35}$ Qiu ZM, Burton, DJ. Preparation of fluorinated 1,2- and $\alpha, \omega$-Diols. J Org Chem. 1993;58:419-423.

${ }^{36}$ Qiu ZM, Burton, DJ. Synthesis of $\alpha, \alpha$-difluoro-functionalized ketones. J Org Chem. 1995;60(17): 5570-5578.

${ }^{37}$ Motoda D, Kinoshita H, Shinokubo H, Oshima K. A room temperature kharasch reaction catalyzed by $\operatorname{Pd}(0)$ in a heterogeneous aqueous system. Adv Synth Catal. 2002;344:261-265. 
${ }^{38}$ Matsubara S, Mitani M, Utimoto K, A facile preparation of 1-perfluoroalkenes and alkynes palladium catalyzed reaction of perfluoroalkyl odides with organotin compounds. Tetrahedron Lett. 1987;28:5857-5860.

${ }^{39}$ Shimizu R, Fuchikami T. Palladium catalyzed coupling reactions of beta-perfluoroalkylsubstituted alkyl halides with organostannanes. Tetrahedron Lett. 1996; 37(46): 8405-8408.

${ }^{40}$ Lanteri MN, Rossi RA, Martín SE. Perfluoroalkylphosphines and arsines obtained by Pdcatalyzed cross-coupling reaction with organoheteroatom stannanes. J Organomet Chem. 2009;694:3425-3430.

${ }^{41}$ Bonaterra BM, Martin SE, Rossi RA. Palladium-catalyzed phenyl-selenylation with n$\mathrm{Bu}_{3} \mathrm{SnSePh}$ in one-pot two-step reactions. Tetrahedron Lett. 2006;47:3511-3515.

${ }^{42}$ Uberman PM, Lanteri MN, Parajón Puenzo SC, Martín SE. Highly efficient palladiumcatalyzed arsination. Synthesis of a biphenyl arsine ligand and its application to obtain perfluoroalkylarsine. Organometallics. 2009;28:6927-6934.

${ }^{43}$ Uberman PM, Lanteri MN, Parajón Puenzo SC, Martín SE. Synthesis of biphenyl-based arsine ligands by Suzuki-Miyaura coupling and their application to Pd-catalyzed arsination. Dalton Trans. 2011;40:9229-9237.

${ }^{44}$ Kitazume, T.; Ishikawa, N. Palladium-catalyzed cross-coupling reactions between allyl, vinyl or aryl halide and perfluoroalkyl iodide with zinc and ultrasonic irradiation. Chem Lett. 1982:137-140.

${ }^{45}$ Kitazume T, Ishikawa N. Ultrasound-promoted pelective perfluoroalkylation on the desired position of organic molecules. J Am Chem Soc.1985;107:5186-5191.

${ }^{46}$ (a) Kitazume T, Ishikawa N. Trifluoromethylation of carbonyl compounds with trifluoromethylzinc iodide under ultrasonic irradiation. Chem Lett.1981:1679-1680.

${ }^{47}$ Filippini L, Gusmeroli M, Riva R. Palladium-catalyzed cross-coupling of pyrrolyl anions with organic halides. Tetrahedron Lett. 1992;33(13):1755-1758.

${ }^{48}$ (a) Kakino R, Shimizu I, Yamamoto A. Synthesis of trifluoromethyl ketones by palladiumcatalyzed cross-coupling reaction of phenyl trifluoroacetate with organoboron compounds. Bull Chem Soc Jpn. 2001;74:371-376. (b) Li C-L, Chen M-W, Zhang X-G, Synthesis of Naryl trifluoromethylarylketoimines by palladium-catalyzed Suzuki coupling reaction of Naryltrifluoroacetimidoyl chlorides with aryl boronic acids. J Fluorine Chem. 2010;131:856860.

${ }^{49}$ Liang A, Li X, Liu D, et al. The palladium-catalyzed cross-coupling reactions of trifluoroethyl iodide with aryl and heteroaryl boronic acid esters. Chem Commun. 2012, 48 : 8273-8275.

${ }^{50}$ (a) Yang CT, Zhang ZQ, Liu YC, Liu L. The palladium-catalyzed cross-coupling reactions of trifluoroethyl iodide with aryl and heteroaryl boronic acid esters. Angew. Chem., Int. Ed., 2011, 50, 3904-3907. (b) Hajipour AR, Nazemzadeh S H, Mohammadsaleh F, Choline chloride $/ \mathrm{CuCl}$ as an effective homogeneous catalyst for palladium-free Sonogashira crosscoupling reactions, Tetrahedron Lett. 2014;55(3),654. (c) Gurung SK, Thapa S, Kafle A, Dickie DA, Giri R. Copper-Catalyzed Suzuki-Miyaura Coupling of Arylboronate Esters: Transmetalation with (PN)CuF and Identification of Intermediates. Org Lett, 2014;16(4):1264-1267. (d) Yang C-T, Zhang Z-O, Tajuddin H, Wu C-C, Liang J, Liu J-H, Fu Y, Czyzewska M, Steel PG, Marder TB, Liu L, Alkylboronic Esters from CopperCatalyzed Borylation of Primary and Secondary Alkyl Halides and Pseudohalides. Angew Chem Int Ed., 2012;124(2):528-532.

${ }^{51}$ Zhao Y, Hu J. Palladium-Catalyzed 2,2,2-Trifluoroethylation of Organoboronic Acids and Esters Angew Chem Int Ed. 2012;51:1033-1036.

${ }^{52}$ Leng F, Wang Y, Li H, et. al. Facile synthesis of trifluoroethyl compounds by the Suzuki cross-coupling reactions of $\mathrm{CF}_{3} \mathrm{CH}_{2} \mathrm{OTs}$ with arylboronic acids. Chem Commun. 2013;49: 10697-10699.

${ }^{53}$ For selected examples of copper catalyzed difluoromethylation see: a) Taguchi T, Kitagawa O, Morikawa T, et. al. Synthesis of 2,2-difluoroesters by iododifluoroacetate-copper with organic halides. Tetrahedron Lett. 1986;27:6103-6106; b) Sato K, Omote M, Ando A, 
Kumadaki I, Reactions of ethyl bromodifluoroacetate in the presence of copper powder. $J$. Fluorine Chem. 2004;125:509-515; c) Zhu J, Zhang W, Zhang L, Liu J, Zheng J, Hu J. Copper-mediated fluoroalkylation reactions with Iododifluoroacetamides: Controlling the selectivity among cross-coupling, intramolecular cyclization, and homocoupling reactions. $J$. Org. Chem. 2010;75:5505-5512; d) Qiu W, Burton DJ, A facile and general preparation of $\alpha, \alpha$-difluoro benzylic phosphonates by the $\mathrm{CuCl}$ promoted coupling reaction of the (diethylphosphonyl)difluoromethylcadmium reagent with aryl lodides. Tetrahedron Lett. 1996;37:2745-2748; e) Yokomatsu T, Murano T, Suemune K, Shibuya S. A direct and practical approach for the synthesis of $\mathrm{Au}(\mathrm{I})$-NHC complexes from commercially available imidazolium salts and Au(III) salts Tetrahedron 1997;53:815-818; f) Jiang X, Chu L, Qing F-L. Copper-mediated oxidative difluoromethylenation of aryl boronic acids with $\alpha$ silyldifluoromethylphosphonates: a new method for aryldifluorophosphonates. New J Chem. 2013;37:1736-1741; (g) Fujikawa K, Fujioka Y, Kobayashi A, Amii H. A new method for aromatic difluoromethylation: Copper-catalyzed cross-coupling and decarboxylation sequence from aryl iodides. Org Lett. 2011;13:5560-5563; h) Feng Z, Chen F, Zhang X. Copper catalyzed cross-coupling of iodobenzoates with bromozinc-difluorophosphonate. Org Lett. 2012;14:1938-1941.

${ }^{54}$ Feng Z, Min Q-Q, Xiao Y-L, Zhang B, Zhang X. Palladium-Catalyzed Difluoroalkylation of Aryl Boronic Acids: A New Method for the Synthesis of Aryldifluoromethylated Phosphonates and Carboxylic Acid Derivatives. Angew Chem Int Ed. 2014; 53: 1669-1673.

${ }^{55}$ Min Q-Q, Yin Z, Feng Z, Guo W-H, Zhang X. Highly selective gem-difluoroallylation of organoborons with bromodifluoromethylated alkenes catalyzed by palladium $J$ Am Chem Soc 2014;136:1230-1233.

${ }^{56}$ Guo C, Yue X, Qing F-L. Palladium-catalyzed cross-coupling of ethyl alpha-bromo-alphafluoroacetate with arylboronic acids: Facile Synthesis of alpha-aryl-alpha-fluoroacetates. Synthesis. 2010;11:1837-1844.

${ }^{57}$ Ruppert I, Schlich K, Volbach W. Die ersten $\mathrm{CF}_{3}$-substituierten organyl(chlor)silane. Tetrahedron Lett.1984;25:2195.

${ }^{58}$ Prakash GKS, Yudin AK. Perfluoroalkylation with organosilicon reagents. Chem Rev. 1997;97:757-786.

${ }^{59}$ Dilman AD, Levin VV. Nucleophilic trifluoromethylation of C=N bonds. Eur J Org Chem. 2011:831-841.

${ }^{60}$ Cho, E. J.; Senecal, T. D.; Kinzel, T.; Zhang, Y.; Watson, D. A.; Buchwald, S. L. The palladium catalyzed trifluoromethylation of aryl chlorides. Science. 2010;328:1679-1681.

${ }^{61}$ Cho EJ, Buchwald SL. The Palladium-Catalyzed Trifluoromethylation of Vinyl Sulfonates. Org Lett. 2011;13:6552-6555.

${ }^{62}$ Tomashenko OA, Grushin VV. Aromatic Trifluoromethylation with Metal Complexes. Chem Rev. 2011;111:4475-4521.

${ }^{63}$ Chen Q, Wu S. Methyl fluorosulphonyldifluoroacetate; a new trifluoromethylating agent. J Chem Soc, Chem Commun. 1989:705.

${ }^{64}$ Roche AJ, Dolbier WR Jr. Electrophilic substitution of 1,1,2,2,9,9,10,10octafluoro[2.2]paracyclophane. J Org Chem. 1999;64:9137-9143.

${ }^{65}$ Roche AJ, Dolbier WR Jr., Jr. Multiple Electrophilic Substitution of 1,1,2,2,9,9,10,10octafluoro[2.2]paracyclophane. J Org Chem. 2000;65:5282-5290.

${ }^{66}$ Previously to the $\mathrm{Cu} / \mathrm{Pd}$ cocatalyzed trifluoromethylation, Liu and Chen had reported the perfluoroalkylation reaction using perfluoroalkyliodides in the presence of metallic copper as reagents. See: Liu C, Chen Q-Y. Practical and efficient synthesis of meso- and $\beta$ perfluoroalkylated porphyrins via Pd-Catalyzed cross-coupling reaction. Synlett. 2005;8:1306-1310.

${ }^{67}$ Liu C, Chen QY. Fluoroalkylation of porphyrins: a facile synthesis of trifluoromethylated porphyrins by a palladium-catalyzed cross-coupling reaction. Eur J Org Chem. 2005;36803686.

${ }^{68}$ Liu C, Shen D-M, Chen Q-Y. Practical and efficient synthesis of various meso-functionalized 
porphyrins via simple ligand-free nickel-catalyzed C-O, C-N, and C-C cross-coupling reactions. J Org Chem. 2007;72:2732-2736.

${ }^{69} \mathrm{Du}$ X, Chen X, Mihalic JT, et al. Design and optimization of imidazole derivatives as potent CXCR3 antagonists. Bioorg Med Chem Lett. 2008;18:608-613.

${ }^{70}$ Qing FL, Zhang X, Peng Y. The trifluoromethylation of 1,1-dibromo-1-alkenes using trifluoromethylcopper $\left(\mathrm{CF}_{3} \mathrm{Cu}\right)$ generated in situ from methylfluorosulfonyldifluoroacetate. $J$ Fluorine Chem. 2001;111:185-187.

${ }^{71}$ For catalytic processes including the activation of $\mathrm{C}-\mathrm{X}$ bonds in trifluoromethylation reactions see (a) Yang CT, Zhang Z-Q, Liu Y-C, Liu L. Copper-Catalyzed Cross-Coupling Reaction of Organoboron Compounds with Primary Alkyl Halides and Pseudohalides. Angew Chem Int. Ed. 2011;50:3904-3907. (b) Gurung SK, Thapa S, Kafle A, Dickie DA, Giri R, Copper-Catalyzed Suzuki-Miyaura Coupling of Arylboronate Esters: Transmetalation with $(\mathrm{PN}) \mathrm{CuF}$ and Identification of Intermediates, Organic Letters, 2014;16:1264 and references therein.

${ }^{72}$ Komatsu Y, SakamotoT, Kitazume T. Synthetic applications of the carbanion with a fluoroalkyl group generated by palladium(0) catalyst under ceutral conditions. J Org Chem. 1999;64:8369-8374.

${ }^{73}$ Zhang W, Zhao Y, Ni C, Mathew T, Hu J. Palladium-catalyzed allylation of 2,2,2trifluoroethyl phenyl sulfone, a potential 2,2,2-trifluoroethyl pronucleophile. Tetrahedron Lett. 2012; 53: 6565-6568.

${ }^{74}$ Fukuzumi T, Shibata N, Sugiura M, Yasui H, Nakamura S, Toru T. Fluorobis(phenylsulfonyl)methane: A fluoromethide equivalent and palladium-catalyzed enantioselective allylic monofluoromethylation. Angew Chem Int Ed. 2006;45:4973-4977.

${ }^{75} \mathrm{Ni}$ C, Hu J. Palladium/acetic acid-catalyzed fluoroalkylation of alkynes with monofluorinated sulfones as pronucleophiles Tetrahedron Lett. 2009;50(52),7252-7255.

${ }^{76}$ Loy R, Sanford MS. Palladium-catalyzed C-H perfluoroalkylation of arenes. Org Lett. 2011;13(10):2548-2551.

${ }^{77}$ Feng Y-S, Xie C-Q, Qiao W-L, Xu H-J. Palladium-catalyzed trifluoroethylation of terminal alkynes with 1,1,1-trifluoro-2-iodoethane. Org Lett. 2013;15(4):936-939.

${ }^{78}$ (a) Eisenberger P, Gischig S, Togni A, Novel 10-I-3 Hypervalent Iodine-Based Compounds for Electrophilic Trifluoromethylation. Chem Eur J. 2006;12:2579-2586. (b) Kieltsch I, Eisenberger P, Togni A. Mild Electrophilic Trifluoromethylation of Carbon- and SulfurCentered Nucleophiles by a Hypervalent Iodine(III)-CF ${ }_{3}$ Reagent. Angew Chem Int Ed. 2007;46:754-757.

${ }^{79}$ For selected reports on trifluoromethylation using Togni s reagent, see: a) Matoušek V, Togni A, Bizet V, Cahard D. Synthesis of $\alpha-\mathrm{CF}_{3}-$ Substituted Carbonyl Compounds with Relative and Absolute Stereocontrol Using Electrophilic $\mathrm{CF}_{3}$-Transfer Reagents. $\mathrm{Org}$ Lett. 2011;13:5762-5765. (b) Niedermann K, Früh N, Senn R, Czarniecki B, Verel R, Togni A. Direct Electrophilic N-Trifluoromethylation of Azoles by a Hypervalent Iodine Reagent. Angew Chem Int Ed. 2012;51:6511-6515 and references therein.

${ }^{80}$ Wang X, Truesdale L, Yu J-Q. Pd(II)-catalyzed ortho-trifluoromethylation of arenes using tfa as a promoter. J Am Chem Soc. 2010;132:3648-3649.

${ }^{81}$ Zhang X-G, Dai H-X, Wasa M, Yu J-Q, Pd(II)-catalyzed ortho trifluoromethylation of arenes and insights into the coordination mode of acidic amide directing groups. $J$ Am Chem Soc. 2012;134:11948-11951.

${ }^{82} \mathrm{Mu}$ X, Chen S, Zheng X, Liu G. Palladium-Catalyzed Oxidative Trifluoromethylation of Indoles at Room Temperature. Chem Eur J. 2011;17:6039-6042.

${ }^{83} \mathrm{Mu}$ X, Wu T, Wang H, Guo Y, Liu G. Palladium-catalyzed oxidative aryltrifluoromethylation of activated alkenes at room temperature. $J$ Am Chem Soc. 2012;134:878-881.

${ }^{84}$ Filippini L, Gusmeroli M, Riva R. Palladium-Catalyzed Coupling of Organic Halides and Tertiary Allylic Amines. Tetrahedron Lett. 1993;34;1643-1646.

${ }^{85}$ Surapanich N, Kuhakarn C, Pohmakotr M, Reutrakul V. Palladium-mediated Heck-type reactions of [(bromodifluoromethyl)-sulfonyl]benzene: Synthesis of $\alpha$-alkenyl- and $\alpha$ - 
heteroaryl-substituted $\alpha, \alpha$-difluoromethyl phenyl sulfones Eur J Org Chem. 2012:59435952.

${ }^{86}$ Albéniz AC, Espinet P, Martín-Ruiz B, Mistein D. Catalytic system for Heck reactions involving insertion into Pd-(perfluoro-organyl) bonds. J Am Chem Soc. 2001;123:1150411505.

${ }^{87}$ Albéniz AC, Espinet P, Martín-Ruiz B, Mistein D. Catalytic system for the Heck reaction of fluorinated haloaryls. Organometallics 2005;24:3679-3684.

${ }^{88}$ Darses S, Pucheault M, Genêt JP. Efficient access to perfluoroalkylated aryl compounds by Heck reaction. Eur. J Org Chem. 2001;1121-1128.

${ }^{89} \mathrm{Chen} \mathrm{W,} \mathrm{Xu} \mathrm{L,} \mathrm{Xiao} \mathrm{J.} \mathrm{A} \mathrm{general} \mathrm{method} \mathrm{to} \mathrm{fluorous} \mathrm{ponytail-substituted} \mathrm{aromatics.}$ Tetrahedron Lett. 2001;42:4275-4278.

${ }^{90}$ Feng J, Cai C. An efficient synthesis of perfluoroalkenylated aryl compounds via Pincer-Pd catalyzed Heck couplings. J Fluorine Chem. 2013;146:6-10.

${ }^{91}$ Von Werner K. Palladium-catalyzed phenylation of allylic 1-perfluoroalkyl alcohols. $J$. Organomet. Chem. 1977;136:385-387.

${ }^{92}$ Csapó A, Rábai J. Siloxane based syntheses of fluorous ethenes and their tandem Heck reactions with aryl iodides. J Fluorine Chem. 2012;144:79-85.

${ }^{93}$ Deeg O, Bäuerle P. Combinatorial organic materials research (COMP): design, synthesis and screening of a 225-membered materials library of liquid crystalline fluorinated $p$ quaterphenyls. Org. Biomol. Chem. 2003;1:1609-1624.

${ }^{94}$ Tannaci JF, Noji M, McBee JL, Tilley TD. 9,10-Disubstituted Octafluoroanthracene Derivatives via Palladium-Catalyzed Cross-Coupling. J. Org. Chem. 2008;73:7895-7900.

${ }^{95}$ Loo YL, Hiszpanski AM, Kim B et al. Unusual molecular conformations in fluorinated, contorted hexabenzocoronenes. Org Lett. 2010;12:4840-4843.

${ }^{96}$ Sharif M, Maalik A, Reimann et at. Synthesis of functionalized fluorinated terphenyls by siteselective Suzuki-Miyaura cross-coupling reactions of dibrominated fluorobenzenes. $J$ Fluorine Chem. 2013;146:19-36.

${ }^{97}$ Liu N, Liu C, Jin Z. Green synthesis of fluorinated biaryl derivatives via thermoregulated ligand/palladium-catalyzed Suzuki reaction. J Organomet Chem. 2011;696:2641-2647.

${ }^{98}$ Korenaga T, Kosaki T, Fukumura R, Ema T, Sakai T. Suzuki-Miyaura coupling reaction using pentafluorophenylboronic acid. Org Lett. 2005;7:4915-4917.

${ }^{99}$ Frohn HJ, Adonin NY, Bardin VV, Starichenko VF. Highly efficient cross-coupling reactions with the perfluoroorganotrifluoroborate salts $\mathrm{K}\left[\mathrm{R}_{\mathrm{F}} \mathrm{BF}_{3}\right]\left(\mathrm{R}_{\mathrm{F}}=\mathrm{C}_{6} \mathrm{~F}_{5}, \mathrm{CF}_{2}=\mathrm{CF}\right)$. Tetrahedron Lett. 2002;43:8111-8114.

${ }^{100}$ Frohn HJ, Adonin NY, Bardin VV, Starichenko VF. A new application of (polyfluoroorgano)trifluoroborate salts: the palladium-catalysed cross-coupling reaction with substituted benzenediazonium tetrafluoroborates. J Fluorine Chem. 2002;117:115-120.

${ }^{101}$ Adonis NY, Babushkin DE, Parmon VN et al. The effect of N-heterocyclic carbene ligands in the palladium-catalyzed cross-coupling reaction of $\left.\mathrm{K}_{2} \mathrm{C}_{6} \mathrm{~F}_{5} \mathrm{BF}_{3}\right]$ with aryl iodides and aryl bromides. Tetrahedron 2008;64:5920-5924.

${ }^{102}$ Robbins DW, Hartwig JF. A C-H borylation approach to Suzuki-Miyaura coupling of typically unstable 2-heteroaryl and polyfluorophenyl boronates. Org Lett. 2012;14:42664269.

${ }^{103}$ Casado AL, Espinet P. Mechanism of the Stille reaction. 1. The transmetalation step. Coupling of $\mathrm{R}^{1} \mathrm{I}$ and $\mathrm{R}^{2} \mathrm{SnBu}$ catalyzed by trans-[PdR $\left.{ }^{1} \mathrm{IL}_{2}\right]\left(\mathrm{R}^{1}=\mathrm{C}_{6} \mathrm{Cl}_{2} \mathrm{~F}_{3} ; \mathrm{R}^{2}=\right.$ Vinyl, 4Methoxyphenyl; $\mathrm{L}=\mathrm{AsPh}_{3}$ ). J Am Chem Soc. 1998;120;8978-8985.

${ }^{104}$ Pérez-Temprano MH, Gallego AM, Casares JA, Espinet P. Stille coupling of alkynyl stannane and aryl iodide, a many-pathways reaction: The importance of isomerization. Organometallics. 2011;30:611-617.

${ }^{105}$ Carrera N, Gutiérrez E, Benavente R, Villavieja MM, Albéniz AC, Espinet P. Stannylated polynorbornenes as new reagents for a clean Stille reaction. Chem Eur J. 2008;14:1014110148. 
${ }^{106}$ Martínez-Arranz S, Carrera N, Albéniz AC, Espinet P, Vidal-Moya A. Batch Stille coupling with insoluble and recyclable stannylated polynorbornenes. Adv Synth Catal. 2012;354:3551-3560.

${ }^{107}$ Carsten B, He F, Son HJ, Xu T, Yu L. Stille polycondensation for synthesis of functional materials. Chem Rev. 2011;111:1493-1528.

${ }^{108} \mathrm{Li} \mathrm{Z}$, Lu J, Tse SC et al. Synthesis and applications of difluorobenzothiadiazole based conjugated polymers for organic photovoltaics. J Mat Chem. 2011;21:3226-3233.

${ }^{109}$ Albeniz AC, Espinet P, Martín-Ruiz B. The Pd-catalyzed coupling of allyl halides and tin aryls: Why the catalytic reaction works and the stoichiometric reaction does not. Chem Eur J. 2001;7:2481-2489.

${ }^{110}$ Chen QY, Li ZT. Palladium-catalyzed alkynyl-dehydroxylation of polyfluorophenols. $J$ Chem Soc Perkin Trans 1. 1992;2931-2934.

${ }^{111}$ Nguyen BV, Yang ZY, Burton DJ. General method for the preparation of functionalized fluorinated phenyl alkynes. J Org Chem. 1993;58:7368-7376.

${ }^{112}$ Collings JC, Burke JM, Smith PS, Batsanov AS, Howard JAK, Marder TB. The synthesis and crystal structures of halogenated tolans $p-\mathrm{X}-\mathrm{C}_{6} \mathrm{H}_{4}-\mathrm{C} \equiv \mathrm{C}-\mathrm{C}_{6} \mathrm{~F}_{5}$ and $p-\mathrm{X}-\mathrm{C}_{6} \mathrm{~F}_{4}-\mathrm{C} \equiv \mathrm{C}-\mathrm{C}_{6} \mathrm{H}_{5}(\mathrm{X}$ = F, Cl, Br, I). Org Biomol Chem. 2004;2:3172-3178.

${ }^{113}$ Bandini M, Luque R, Budarin V, Macquarrie DJ. Aryl alkynylation versus alkyne homocoupling: unprecedented selectivity switch in $\mathrm{Cu}$, phosphine and solvent-free heterogeneous Pd-catalysed couplings. Tetrahedron 2005;61:9860-9868.

${ }^{114}$ Orbach M, Choudhury J, Lahav M et al. Palladium-catalyzed cross-coupling reactions with fluorinated substrates: mechanistic insights into the undesired hydrodehalogenation of aryl halides. Organometallics 2012;31:1271-1274.

${ }^{115}$ Aspin S, Goutierre AS, Larini P, Jazzar R, Baudoin O. Synthesis of aromatic $\alpha$-aminoesters: palladium-catalyzed long-range arylation of primary $\mathrm{Csp}^{3}$-H bonds. Angew Chem Int Ed. 2012;51:10808-10811.

${ }^{116}$ Larini P, Kefalidis CE, Jazzar R, Renaudat A, Clot E, Baudoin O. On the mechanism of the palladium-catalyzed $\beta$-arylation of ester enolates. Chem Eur J. 2012;18:1932-1944.

${ }^{117}$ Yuan K, Boixel J, Le Bozec H, et al. Perfluorocyclohexene bridges in inverse diarylethenes: synthesis through Pd-catalysed $\mathrm{C}-\mathrm{H}$ bond activation, experimental and theoretical studies on their photoreactivity. Chem Commun. 2013;49:7896-7898.

${ }^{118}$ Luo HQ, Dong W, Loh TP. Pd-catalyzed decarboxylative cross-coupling of perfluorobenzoic acids with simple arenes. Tetrahedron Lett. 2013;54:2833-2836.

${ }^{119}$ Lafrance M, Rowley CN, Woo TK, Fagnou K. Catalytic intermolecular direct arylation of perfluorobenzenes. J Am Chem Soc. 2006;128:8754-8756.

${ }^{120}$ Lafrance M, Shore D, Fagnou K. Mild and general conditions for the cross-coupling of aryl halides with pentafluorobenzene and other perfluoroaromatics. Org Lett. 2006;8:5097-5100.

${ }^{121}$ García-Cuadrado D, de Mendoza P, Braga AAC, Maseras F, Echavarren AM. Protonabstraction mechanism in the palladium-catalyzed intramolecular arylation: substituent effects. J Am Chem Soc. 2007;129:6880-6886.

${ }^{122}$ Gorelski SI, Lapointe D, Fagnou K. Analysis of the concerted metalation-deprotonation mechanism in palladium-catalyzed direct arylation across a broad range of aromatic substrates. J Am Chem Soc. 2008;130:10848-10849.

${ }^{123}$ Fan S, Yang J, Zhang X. Pd-catalyzed direct cross-coupling of electron-deficient polyfluoroarenes with heteroaromatic tosylates. Org. Lett. 2011;13:4374-4377.

${ }^{124}$ Fan S, He CY, Zhang X. Direct Pd-catalyzed benzylation of highly electron-deficient perfluoroarenes. Chem Commun. 2010;46:4926-4928.

${ }^{125} \mathrm{Yu}$ YB, Fan S, Zhang X. Copper- and phosphine-ligand-free palladium-catalyzed direct allylation of electron-deficient polyfluoroarenes with allylic chlorides. Chem Eur J. 2012;18:14643-14648.

${ }^{126}$ Fan S, Chen, Zhang X. Direct palladium-catalyzed intermolecular allylation of highly electron-deficient polyfluoroarenes. Angew Chem Int Ed. 2011;50:5918-5923.

${ }^{127} \mathrm{He} \mathrm{CY}$, Wu CZ, Quina FL, Zhang X. Direct (het)arylation of fluorinated benzothiadiazoles 
and benzotriazole with (het)aryl iodides. J Org Chem. 2014;70:1712-1718.

${ }^{128}$ Stuart DR, Fagnou K. The catalytic cross-coupling of unactivated arenes. Science 2007;316:1172-1175.

${ }^{129}$ Wei Y, Su W. Pd(OAc) $)_{2}$-catalyzed oxidative C-H/C-H cross-coupling of electron-deficient polyfluoroarenes with simple arenes. J Am Chem Soc. 2010;132:16377-16379.

${ }^{130} \mathrm{Li} \mathrm{H}$, Liu J, Sun CL, Li BJ, Shi ZJ. Palladium-catalyzed cross-coupling of polyfluoroarenes with simple arenes. Org Lett. 2011;13:276-279.

${ }^{131} \mathrm{He}$ CY, Fan S, Zhang X. Pd-catalyzed oxidative cross-coupling of perfluoroarenes with aromatic heterocycles. J Am Chem Soc. 2010;132:12850-12852.

${ }^{132} \mathrm{He}$ CY, Min QQ, Zhang X. Palladium-catalyzed aerobic dehydrogenative cross-coupling of polyfluoroarenes with thiophenes: facile access to polyfluoroarene-thiophene structure. Organometallics 2012;31:1335-1340.

${ }^{133}$ Fan S, Chen F, Zhang X. Direct palladium-catalyzed intermolecular allylation of highly electron-deficient polyfluoroarenes. Angew Chem Int Ed.2011;50:5918-5923.

${ }^{134} \mathrm{Li}$ Z, Zhang Y, Liu ZQ. Pd-catalyzed olefination of perfluoroarenes with allyl esters. Org Lett. 2012;14:74-77.

${ }^{135}$ Fang X, Huang Y, Chen X et al. Preparation of fluorinated biaryls through direct palladiumcatalyzed coupling of polyfluoroarenes with aryltrifluoroborates. J Fluorine Chem. 2013;151:50-57.

${ }^{136}$ Zhao H, Wei Y, Xu J Kan J, Su W, Hong M. Pd/PR -catalyzed cross-coupling of aromatic carboxylic acids with electron-deficient polyfluoroarenes via combination of decarboxylation with sp ${ }^{2}$ C-H cleavage. J Org Chem. 2011;76:882-893.

${ }^{137}$ Heinze PL, Burton DJ. Palladium-catalyzed cross-coupling of perfluoroalkenylzinc reagents with aryl iodides. A new, simple synthesis of $\alpha, \beta, \beta$-trifluorostyrenes and the stereoselective preparation of 1-arylperfluoropropenes. J Org Chem. 1988;53:2714-2720.

${ }^{138}$ Davis CR, Burton DJ. Stereoselective preparation of $(\mathrm{Z})-\alpha, \beta$-difluorostyrenes. Tetrahedron Lett. 1996;37:7237-7240.

${ }^{139}$ Koroniak H, Karwatka P, Pluskota D, Fiedorow P, Jankowski J. Synthesis of some 5perfluoroalkenyl derivatives of uracil. J Fluorine Chem. 1995;71:135-137.

${ }^{140}$ Shigeoka T, Kuwahara Y, Watanabe K et al. Synthesis of fluorine analogs of protoporphyrin potentially useful for diagnosis and therapy of cancer. IV.1) Synthesis of (trifluorovinyl)vinyl- and (1-chloro-2,2-difluorovinyl)vinyldeuteroporphyrins. Chem Pharm Bull. 1999;47:1326-1329.

${ }^{141}$ Roemer M, Kang YK, Cheng YK, Lentz D. Ferrocenes with perfluorinated side chains and ferrocenophanes with fluorinated handles. Chem Eur J. 2012;18:3371-3389.

${ }^{142}$ Akkerman FA, Kickbusch R, Lentz D. Synthesis of fluorinated dienes by palladiumcatalyzed coupling reactions. Chem Asian J. 2008;3:719-731.

${ }^{143}$ Tellier F, Sauvêtre R, Normant JF. Preparation et reactivite de quelques enynes fluores. Tetrahedron Lett. 1986;27:3147-3148.

${ }^{144}$ Dutheuil G, Paturel C, Lei X, Couve-Bonnaire S, Pannecoucke X. First stereospecific synthesis of $(E)$ - or $(Z)$ - $\alpha$-fluoroenones via a kinetically controlled Negishi coupling reaction. J Org Chem. 2006;71:4316-4319.

${ }^{145}$ Shen Y, Wang G. Stille cross-coupling reaction of polyfluorovinylstannanes Stereospecific synthesis of polyfluoro-alkenes and - $\alpha, \beta$-unsaturated ketones. J Fluorine Chem. 2004;125:91-94.

${ }^{146}$ Wang Y, Lu L, Burton DJ. Stereospecific preparation of (Z)- and (E)-2,3-difluoro-3stannylacrylic ester synthons and a new, efficient stereospecific route to (Z)- and (E)-2,3difluoroacrylic esters. J Org Chem. 2005;70:10743-10746.

${ }^{147}$ Brisdon AK, Pritchard RG, Thomas A. Pentafluoropropenyl complexes of mercury, germanium, tin, and lead derived from $(\mathrm{Z})-\mathrm{CFH}=\mathrm{CFCF}_{3}$ and their use as transfer reagents. Organometallics 2012;31:1341-1348. 
${ }^{148}$ Yamada S, Ishii E, Konno T, Ishihara T. Preparation of perfluorocyclopentenylmetal species and their cross-coupling reaction with electrophilesdRemarkable accesses to versatile perfluorocyclopentene derivatives. Tetrahedron 2008;64:4215-4223.

${ }^{149}$ Lim C, Burton DJ, Wesolowski CA. The stereospecific preparation of two perfluoro-1,3butadiene synthons; (E)-1-trimethylsilyl-1,2,3,4,4-pentafluoro-1,3-butadiene and (E)-1tributylstannyl-1,2,3,4,4-pentafluoro-1,3-butadiene. J Fluorine Chem. 2003;119:21-26.

${ }^{150} \mathrm{Xu}$ J, Burton DJ. Stereospecific preparation of symmetrical (1Z, 3Z)- and (1E, 3E)-2, 3difluoro-1,4-disubstituted-buta-1,3-dienes from 1-bromo-1-fluoroalkenes. J Fluorine Chem. 2007; 128:71-77.

${ }^{151}$ Babudri F, Cardone A, Farinosa GM et al. Synthesis of poly(arylenevinylene)s with fluorinated vinylene units. Eur J Org Chem. 2008;1977-1982.

${ }^{152}$ Cardone A, Martinelli C, Pinto V et al. Synthesis and characterization of perfluorinated arylenevinylene aolymers. J Pol Sci A: Pol Chem. 2010;48:285-291.

${ }^{153}$ Chen C, Wilcoxen K, Strack N, McCarthy JR. Synthesis of fluorinated olefins via the palladium catalyzed cross-coupling reaction of 1-fluorovinyl halides with organoboranes. Tetrahedron Lett. 1999;40:827-830.

${ }^{154}$ Wilson PG, Percy JM, Redmond JM, McCarter AW. Suzuki-Miyaura coupling reactions of iodo(difluoroenol) derivatives, fluorinated building blocks accessible at near-ambient temperaturas. J Org Chem. 2012;77:6384-6393.

${ }^{155}$ Wójtowicz-Rajchel H, Koroniac H. Synthesis of 5-fluorovinyl derivatives of pyrimidines via Suzuki-Miyaura coupling and their 1,3-dipolar cycloaddition reactions with nitrotes. $J$ Fluorine Chem. 2012;135:225-230.

${ }^{156}$ Eddarir S, Francesca C, Mestdagh H, Rolando C. Synthesis of fluorinated enynes via 1bromo l-fluoro alkenes. Tetrahedron Lett. 1990;31:4449-4452.

${ }^{157}$ Yang ZY, Burton DJ. Facile, general method for the preparation of fluorinated enynes. Tetrahedron Lett. 1990;31:1369-1372.

${ }^{158}$ Yang ZY, Burton DJ. Palladium-catalyzed reaction of fluorinated vinyl iodides with terminal alkynes: a new and general route to fluorinated enynes. J Fluorine Chem. 1991;53:307-326.

${ }^{159}$ Zemmouri R, Kajjout M, Castanet Y, Edarir S, Rolando C. Palladium-catalyzed stereoconvergent formylation of (E/Z)- $\beta$-bromo- $\beta$-fluorostyrenes: straightforward access to (Z)- $\beta$-fluorocinnamic aldehydes and (Z)- $\beta$-fluorocinnamic alcohols. J Org Chem. 2011;76:7691-7698.

${ }^{160}$ Zatolochnaya OV, Gevorgyan V. Synthesis of fluoro- and perfluoroalkyl arenes via palladium-catalyzed [4 + 2] benzannulation reaction. Org Lett. 2013;15:2562-2565.

${ }^{161}$ Kawatsura M, Wada S, Hayase S, Itoh T. Palladium-catalyzed regioselective allylic alkylation of 1-aryl-2,3,3-trifluoroallyl acetates. Synlett. 2006;2483-2485.

${ }^{162}$ Bélanger E, Pouliot MF, Courtemanche MA, Paquin JF. Design, synthesis, and applications of potential substitutes of t-Bu-phosphinooxazoline in Pd-catalyzed asymmetric transformations and their use for the improvement of the enantioselectivity in the Pdcatalyzed allylation reaction of fluorinated allyl enol carbonates. J Org Chem. 2012;77:317331.

${ }^{163}$ The crystallographic structure of this complex is available: Grushin VV, Marshall WJ. Unexpected $\mathrm{H}_{2} \mathrm{O}$-induced Ar-X activation with trifluoromethylpalladium(II) aryls. J Am Chem Soc. 2006;128:4632-4641.

${ }^{164}$ Algarra AG, Grushin VV, Macgregor SA. Natural bond orbital analysis of the electronic structure of $\left[\mathrm{L}_{n} \mathrm{M}\left(\mathrm{CH}_{3}\right)\right]$ and $\left[\mathrm{L}_{n} \mathrm{M}\left(\mathrm{CF}_{3}\right)\right]$ complexes Organometallics 2012;31:1467-1476.

${ }^{165}$ Yang D-S, Bancroft GM, Puddephatt RJ, Tses JS. Electronic structure of square-planar cisbis(trifluoromethyl)platinum(II) complexes from UV Photoelectron Spectra and SCF-MS-X Calculations. Inorg Chem. 1990;29:2496-2501.

${ }^{166}$ Sheppard WA. Pentafluorophenyl group. electronic effect as a substituent. J Am Che. Soc. 1970;92:5419-5422.

${ }^{167}$ Clot E, Mégret C, Eisenstein O, Perutz RN. Exceptional sensitivity of metal-aryl bond energies to ortho-fluorine substituents: Influence of the metal, the coordination sphere, and 
the spectator Ligands on M-C/H-C bond energy correlations. J Am Chem Soc. 2009;131:7817-7827.

${ }^{168}$ Guihaumé J, Clot E, Mégret C, Eisenstein O, Perutz RN. Importance of palladium-carbon bond energies in direct arylation of polyfluorinated benzenes. Dalton Trans. 2010;39:1051010519.

${ }^{169}$ Casado AL, Espinet P. On the configuration resulting from oxidative addition of RX to $\operatorname{Pd}\left(\mathrm{PPh}_{3}\right)_{4}$ and the mechanism of the cis-to-trans isomerization of $\left[\mathrm{PdRX}\left(\mathrm{PPh}_{3}\right)_{2}\right]$ complexes $(\mathrm{R}=$ Aryl, $\mathrm{X}=$ Halide). Organometallics 1998;17:954-959.

${ }^{170}$ Orbach M, Zenkina OV, Diskin-Posner Y, Iron MA, van del Boom M. A PhosphineAccelerated $\mathrm{Ar}_{\mathrm{F}}-\mathrm{Ch}$ loride Bond Activation Process by Palladium. Organometallics 2013;32:3074-3082.

${ }^{171}$ Powers DC, Geibel MAL, Klein JENMN, Ritter, T. Bimetallic Palladium Catalysis: Direct Observation of Pd(III)-Pd(III) Intermediates. J Am Chem Soc. 2009;131:17050-17051.

${ }^{172}$ Powers DC, Ritter T. Bimetallic redox synergy in oxidative palladium catalysis Acc Chem Res. 2012;45:840-850.

${ }^{173}$ Ye Y, Ball ND, Kampf JW, Sanford MS. Oxidation of a cyclometalated Pd(II) dimer with " $\mathrm{CF}_{3}{ }_{3}$ ": Formation and reactivity of a catalytically competent monomeric $\mathrm{Pd}(\mathrm{IV})$ aquo complex. J Am Chem Soc. 2010;132:14682-14687.

${ }^{174}$ Racowski JM, Ball ND, Sanford MS. C-H Bond activation at palladium(IV) centers. J Am Chem Soc. 2011;133:18022-18025.

${ }^{175}$ Casado AL, Casares JA, Espinet P. An aryl exchange reaction with full retention of configuration of the complexes: Mechanism of the aryl exchange between $\left[\mathrm{PdR}_{2} \mathrm{~L}_{2}\right]$ complexes in chloroform $(\mathrm{R}=$ Pentahalophenyl, $\mathrm{L}=$ Thioether $)$. Organometallics . 1997; 16:5730-5736.

${ }^{176}$ Pérez-Temprano MH, Nova A, Casares JA, Espinet P. Observation of a hidden intermediate in the Stille reaction. Study of the reversal of the transmetalation step. J Am Chem Soc. 2008;130(32):10518-10519.

${ }^{177}$ Casado AL, Espinet P. Quantitative evaluation of the factors contributing to the "Copper Effect" in the Stille reaction. Organometallics 2003;22:1305-1309.

${ }^{178}$ Casado AL, Espinet P, Gallego AM. Mechanism of the Stille reaction. 2. Couplings of aryl triflates with vinyltributyltin. Observation of intermediates. A more comprehensive scheme $J$ Am Chem Soc. 2000;122:11771-11782.

${ }^{179}$ Espinet P, Echavarren AM. The mechanisms of the Stille reaction. Angew Chem Int Ed. 2004;43:4704-4734.

${ }^{180}$ Casado AL, Espinet P, Gallego AM, Martínez-Ilarduya JM. Snapshots of a Stille reaction. Chem Commun. 2001:339-340.

${ }^{181}$ Casado AL, Espinet P. A novel reversible aryl exchange involving two organometallics: Mechanism of the gold(I)-catalyzed isomerization of trans-[PdR $\left.\mathrm{PL}_{2}\right]$ complexes $(\mathrm{R}=\mathrm{Aryl}, \mathrm{L}$ $=\mathrm{SC}_{4} \mathrm{H}_{8}$ ) Organometallics. 1998; 17:3677-3683.

182 delPozo J, Carrasco D, Pérez-Temprano MH, García-Melchor M, Álvarez R, Casares JA, Espinet P. Stille coupling involving bulky groups feasible with gold cocatalyst. Angew Chem Int Ed. 2013;52:2189-2193.

${ }^{183}$ delPozo J, Casares JA, Espinet P. The decisive role of ligand metathesis in Au/Pd bimetallic catalysis. Chem Commun. 2013;49:7246-7248.

${ }^{184}$ Pérez-Temprano MH, Casares JA, de Lera AR; Álvarez R. Espinet P. Strong metallophilic interactions in the palladium arylation by gold aryls. Angew Chem Int Ed. 2012;20:49174920.

${ }^{185}$ Casares JA, Espinet P, Fuentes B, Salas G. Insights into the mechanism of the Negishi reaction: ZnRX versus $\mathrm{ZnR}_{2}$ reagents. $J$ Am Chem Soc. 2007;129:3508-3509.

${ }^{186}$ For retro-transmetalation processes and group exchange between palladium and zinc see also: (a) Fuentes B, Garcia-Melchor M, Lledós A. et al. Palladium round trip in the Negishi coupling of trans-[PdMeCl$\left.\left(\mathrm{PMePh}_{2}\right)_{2}\right]$ with $\mathrm{ZnMeCl}$. An experimental and DFT study of the transmetallation step. Chem Eur J. 2010;16:8596-8599. (b) Garcia-Melchor M, Fuentes B, Lledós A, Casares JA, Ujaque G, Espinet P. Cationic intermediates in the Pd-catalyzed 
Negishi coupling. Kinetic and Density Functional Theory study of alternative transmetalation pathways in the Me-Me coupling of $\mathrm{ZnMe}_{2}$ and trans-[PdMeCl$\left.\left(\mathrm{PMePh}_{2}\right)_{2}\right] J$ Am Chem Soc. 2011;133(34):13519-13526.

${ }^{187}$ The exchange of organic groups between organozinc and palladium complexes have been reported: van Asselt, R.; Elsevier, C. J. Rigid bidentate nitrogen ligands in organometallic chemistry and homogeneous catalysis. 8. On the mechanism of formation of homocoupled products in the carbon-carbon cross-coupling reaction catalyzed by palladium complexes containing rigid bidentate nitrogen ligands: Evidence for the exchange of organic groups between palladium and the transmetalating reagent. Organometallics. 1994;13:1972-1980.

${ }^{188}$ The aryl-by aryl exchange between organozinc and palladium complexes has also been reported: Liu Q, Lan Y, Liu J, Li G, Wu YD, Lei A, Revealing a second transmetalation step in the Negishi coupling and its competition with reductive elimination: Improvement in the interpretation of the mechanism of biaryl syntheses. J Am Chem Soc. 2009;131:1020110210.

${ }^{189}$ Hughes PR, Overby JS, Williamson A, Lam K-C, Concolino TE, Rheingold AL. Synthesis, molecular structures, and dynamics of primary and secondary fluoroalkyl complexes of palladium(II) with tetramethylethylenediamine (TMEDA) ligands. Evaluation of the structural trans-influences of methyl and fluoroalkyl groups as ligands within the same coordination sphere. Organometallics. 2000;19:5190-5201.

${ }^{190}$ Hughes RP, Meyer MA, Tawa MD, Ward AJ, Williamson A, Rheingold AL, Zakharov LN. Does $\alpha$-fluorination affect the structural trans-influence and kinetic trans-effect of an alkyl ligand? Molecular structures of $\mathrm{Pd}(\mathrm{TMEDA})\left(\mathrm{CH}_{3}\right)(\mathrm{RF})$ and a kinetic study of the trans to cis isomerization of $\mathrm{Pt}(\mathrm{TMEDA})\left(\mathrm{CH}_{3}\right)_{2} \mathrm{I}(\mathrm{RF})\left[\mathrm{RF}=\mathrm{CF}_{2} \mathrm{CF}_{3}, \mathrm{CFHCF}_{3}, \mathrm{CH}_{2} \mathrm{CF}_{3}\right]$ Inorg Chem. 2004;43:747-756.

${ }^{191}$ Culkin DA, Hartwig JF. Carbon-carbon bond-forming reductive elimination from arylpalladium complexes containing functionalized alkyl groups. Influence of ligand steric and electronic properties on structure, stability, and reactivity. Organometallics. 2004;23:3398-3416.

${ }^{192}$ Grushin VV, Marshall WJ. Facile $\mathrm{Ar}_{-} \mathrm{CF}_{3}$ bond formation at Pd. Strikingly different outcomes of reductive elimination from $\left[\left(\mathrm{Ph}_{3} \mathrm{P}\right)_{2} \mathrm{Pd}\left(\mathrm{CF}_{3}\right) \mathrm{Ph}\right]$ and $\left[(\mathrm{Xantphos}) \mathrm{Pd}\left(\mathrm{CF}_{3}\right) \mathrm{Ph}\right] . J$ Am Chem Soc. 2006;128:12644-12645.

${ }^{193}$ The effect of the bite angle on the reductive elimination processes has been profusely studied. The issue has been recently reviewed: Birkholz M-N, Freixa Z, van Leeuwen PWNM. Bite angle effects of diphosphines in $\mathrm{C}-\mathrm{C}$ and $\mathrm{C}-\mathrm{X}$ bond forming cross coupling reactions. Chem Soc Rev. 2009;38:1099-1118.

${ }^{194}$ Kamer PCJ, van Leeuwen PWNM, Reek JNH. Wide bite angle diphosphines: Xantphos ligands in transition metal complexes and catalysis. Acc Chem Res. 2001;34:895-904.

${ }^{195}$ Bakhmutov VI, Bozoglian F, Gomez K, Gonzalez G, Grushin VV, Macgregor SA, Martin E, Miloserdov FM, Novikov MA, Panetier JA, Romashov LV. $\mathrm{CF}_{3}-\mathrm{Ph}$ reductive elimination from [(Xantphos) $\left.\mathrm{Pd}\left(\mathrm{CF}_{3}\right)(\mathrm{Ph})\right]$. Organometallics 2012;31:1315-1328.

${ }^{196}$ Anstaett P, Schoenebeck F. Reductive elimination of $\mathrm{Ar}-\mathrm{CF}_{3}$ from bidentate $\mathrm{Pd}^{\mathrm{II}}$ complexes: A computational study. Chem Eur J. 2011;17:12340-12346.

${ }^{197}$ For the effect of additives in the reductive elimination and its mechanisms see: PérezRodríguez M, Braga AAC, Garcia-Melchor M, et. al. C-C reductive elimination in palladium complexes, and the role of coupling additives. A DFT study supported by experiment. $J \mathrm{Am}$ Chem Soc. 2009;131:3650-3657 and references therein.

${ }^{198}$ Chaouche N, Fornies J, Fortuño C, Kribii A, Martín A, Karipidis P, Tsipis AC, Tsipis CA. Experimental and quantum chemical study of the mechanism of an unexpected intramolecular reductive coupling of a bridging phosphido ligand and $\mathrm{C}_{6} \mathrm{~F}_{5}$ group and the reversible oxidative addition of $\mathrm{PPh}_{2} \mathrm{C}_{6} \mathrm{~F}_{5}$. Organometallics 2004;23:1797-1810.

${ }^{199}$ Ara I, Chaouche N, Fornies J, Fortuño C, Kribii A, Tsipis AC. Formation of $\mathrm{PPh}_{2} \mathrm{C}_{6} \mathrm{~F}_{5}$ through phosphido platinum and/or palladium(III) intermediates. Organometallics 
2006;25:1084-1091.

${ }^{200}$ Ball ND, Kampf JW, Sanford MS. Aryl- $\mathrm{CF}_{3}$ Bond-Forming Reductive Elimination from Palladium(IV). J Am Chem Soc. 2010;132:2878-2879

${ }^{201}$ Ball ND, Gary JB, Ye Y, Sanford MS. Mechanistic and computational studies of oxidativelyinduced Aryl- $-\mathrm{CF}_{3}$ bond-formation at Pd: Rational design of room temperature aryl trifluoromethylation. J Am Chem Soc. 2011;133:7577-7584.

${ }^{202}$ Albeniz AC, Espinet P, Foces-Foces C, Cano FH. "Pd $\left(\mathrm{C}_{6} \mathrm{~F}_{5}\right) \mathrm{Br}$ ", a convenient precursor for studying the endo attack of nucleophiles on olefins. X-ray Structure of bis(p-bromo)bis(4(pentafluoropheny1)-1-3- $\eta^{3}$-cyc1ohexenyl)dipalladium(II). Organometallics 1990;9:10791085.

${ }^{203}$ Albeniz AC, Espinet P. On the requirements of the precursor complex for olefin insertion: reactivity of cis- and trans- $\mathrm{Pd}\left(\mathrm{C}_{6} \mathrm{~F}_{5}\right)(\mathrm{L})_{2}{ }^{+}$with dienes. J. Organomet. Chem. 1993;452:229234.

${ }^{204}$ Albeniz AC, Espinet P, Jeannin Y, Philoche-Levisalles M, Mann B, Synthesis and subsequent rearrangement of chloro(pentafluoropheny1)-1,5-cyclooctadienepalladium(II), an Illustrative example of endo attack to a coordinated double bond. J. Am. Chem. Soc. 1990;112:6594-6600.

${ }^{205}$ Albéniz AC, Espinet P, Lin YS. Regioselectivity of the insertion of dienes into Pd-R bonds. diastereoselection in the isomerization of an $\left(\eta^{1}-\eta^{2}\right.$-enyl)palladium complex to an $\left(\eta^{3}\right.$ allyl)palladium complex. Organometallics 1995;14:2977-2986.

${ }^{206}$ Albéniz AC, Espinet P. A case of slow isomerization of a ( $\sigma-\pi$-hexeny1)palladium complex and its relevance to organic synthesis. Organometallics 1991;10:2987-2988.

${ }^{207}$ Albéniz AC, Espinet P, Lin YS. Palladium migration along linear carbon chains: the detection of $\left(\eta^{1}-\eta^{2}\right.$-enyl) intermediates and the study of their rearrangement. Organometallics 1997; 16:4138-4144.

${ }^{208}$ Albéniz AC, Espinet P, Lin YS. Involvement of intramolecular hydride transfer in the formation of alkanes from palladium alkyls. Organometallics 1997;16:4030-4032.

${ }^{209}$ Albéniz AC, Espinet P, Lin YS, Martín Ruiz B. "Gated migration” for enantioselective synthesis of palladium allyls using a "PdHBr" synthon. Organometallics 1999;16:33593363.

${ }^{210}$ Albéniz AC, Espinet P, López-Fernández R, Sen A. A warning on the use of radical traps as a test for radical mechanisms: they react with palladium hydrido complexes $J$ Am Chem Soc. 2002;124:11278-11279.

${ }^{211}$ Molina de la Torre JA, Espinet P, Albeniz AC. Solvent-induced reduction of palladium-aryls, a potential interference in Pd catalysis. Organometallics 2013;32:5428-5434.

${ }^{212}$ Albéniz AC, Espinet P, Lin YS. Study of the evolution of $\left(\eta^{1}-\eta^{2}\right.$-enylpalladium complexes when the palladium-migration process is blocked. Organometallics 1997;16:5964-5973.

213 (a) Albéniz AC, Espinet P, Lin YS, Orpen AG, Martín A. Insertion of alkenyl sulfides into a palladium-aryl bond. 1. Synthesis and evolution of a three-membered thiopalladacycle. Xray crystal structure of a new tetrameric palladium derivative with bridging (phenylthio)alkyl ligands. Organometallics 1996;15:5003-5009. (b) Albéniz AC, Espinet P, Lin YS. Insertion of alkenyl sulfides into a palladium-aryl bond. 2. Stabilization of $\sigma$-yl-kS chelates and decomposition reactions through C-S cleavage. Organometallics 1996;15:5010-5017.

${ }^{214}$ Albéniz AC, Espinet P, Lin YS. Cyclization versus Pd-H elimination-eeaddition: Skeletal rearrangement of the products of $\mathrm{Pd}-\mathrm{C}_{6} \mathrm{~F}_{5}$ addition to 1,4-pentadienes. $J$ Am Chem Soc. 1996;118:7145-7152.

${ }^{215}$ Albéniz AC, Espinet P, López-Fernández R. Competition of insertion and transmetalation pathways in the reactions of alkenylsilanes with aryl complexes of palladium(II). An experimental study. Organometallics 2006;25:5449-5455.

${ }^{216}$ Kashiwabara T, Tanaka M. Decarbonylative coupling of fluorobenzoyl chlorides with hexamethyldisilane in the presence of a palladium complex catalyst: Extremely facile decarbonylation of pentafluorobenzoyl-Pd complex relevant to $\mathrm{C}_{6} \mathrm{~F}_{5} \mathrm{SiMe}_{3}$ formation. Organometallics 2006;25:4648-4652. 
${ }^{217}$ Usón R, Forniés J, Espinet P, Lalinde E. Reactions of $\left[\mathrm{Pd}\left(\mathrm{C}_{6} \mathrm{~F}_{5}\right)_{2}(\mathrm{CNR})_{2}\right]$ with $\left[\mathrm{PdCl}_{2}(\mathrm{NCPh})_{2}\right]$. Insertion of p- $\mathrm{MeC}_{6} \mathrm{H}_{4} \mathrm{NC}$ into $\mathrm{Pd}-\mathrm{C}_{6} \mathrm{~F}_{5}$ bonds. J Organomet Chem . 1983;254:371-379.

${ }^{218}$ Usón R, Forniés J, Espinet P, Lalinde E, Jones PG, Sheldrick GM. Terminal Pentafluorobenzimidoylpalladium(II) complexes. X-ray structure of trans$\left.\left[\mathrm{Pd}\left\{\mathrm{C}_{6} \mathrm{~F}_{5}\right)=\mathrm{NMe}\right\} \mathrm{Cl}(\mathrm{CNMe})_{2}\right]$. J Organomet Chem. 1985;288:249-259.

${ }^{219}$ See for example: Barluenga J, Valdés C. Tosylhydrazones: New uses for classic reagents in palladium-catalyzed cross-coupling and metal-free reactions. Angew Chem Int Ed. 2011;50:7486-7500.

${ }^{220}$ Albéniz AC, Espinet P, Manrique R, Pérez-Mateo A. Observation of the direct products of migratory insertion in aryl palladium carbene complexes and their subsequent hydrolysis. Angew Chem Int Ed. 2002;41:2363-2366.

${ }^{221}$ Albéniz AC, Espinet P, Manrique R, Pérez-Mateo A. Aryl palladium carbene complexes and carbene-aryl coupling reactions. Chem Eur J. 2005;11:1565-1573.

${ }^{222}$ Albéniz AC, Espinet P, Pérez-Mateo A, Nova A, Ujaque G. Formation of a Vinyliminium Palladium Complex by C-C Coupling in Vinylcarbene Palladium Aryl Complexes. Organometallics 2006;25:1293-1297.

${ }^{223}$ Wang X, Xu Y, Deng Y, et al. Pd-carbene migratory insertion: application to the synthesis of trifluoromethylated alkenes and dienes. Chem. Eur. J. 2014;20:961-965.

${ }^{224}$ Nova A, Mas-Ballesté R, Lledós A. Breaking C-F bonds via nucleophilic attack of coordinated ligands: transformations from $\mathrm{C}-\mathrm{F}$ to $\mathrm{C}-\mathrm{X}$ bonds $(\mathrm{X}=\mathrm{H}, \mathrm{N}, \mathrm{O}, \mathrm{S})$. Organometallics 2012;31:1245-1256.

${ }^{225}$ Sun AD, Love JA. Cross coupling reactions of polyfluoroarenes via C-F activation. Dalton Trans. 2010;39:10362-10364.

${ }^{226}$ Kiplinger JL, Richmond TG, Osterberg CE, Activation of carbon-fluorine bonds by metal complexes. Chem Rev. 1994;94:373-431.

${ }^{227}$ Grushin VV. The organometallic fluorine chemistry of palladium and rhodium: studies toward aromatic fluorination. Acc Chem Res. 2010;43:160-171.

${ }^{228}$ Vigalok A. Electrophilic fluorination of group 10 organometallic complexes: Chemistry beyond oxidative addition. Organometallics 2011;30:4802-4810.

229 (a) Widdovson DA, Wilhelm R. Palladium catalysed cross-coupling of (fluoroarene)tricarbonylchromium(0) complexes. Chem Commun. 1999;2211-2212. (b)

Wilhelm R, Widdovson DA. Palladium catalysed cross-coupling of (fluoroarene)tricarbonylchromium(0). J Chem Soc Perkin Trans. 1. 2000;3808-3813.

${ }^{230}$ Kim YM, Yu S. Palladium(0)-catalyzed amination, Stille coupling, and Suzuki coupling of electron-deficient aryl fluorides. J Am Chem Soc. 2003;125:1696-1697.

${ }^{231}$ Widdovson DA, Wilhelm R. Palladium catalysed Suzuki reactions of fluoroarenes. Chem Commun. 2003;578-579

${ }^{232}$ Mikami K, Miyamoto T, Hatao M. A highly efficient asymmetric Suzuki-Miyaura coupling reaction catalyzed by cationic chiral palladium(II) complexes. Chem Commun. 2004; 20822083

${ }^{233}$ Cargill MR, Sandford G, Tadeusiak AJ, et al. Palladium-catalyzed C-F activation of polyfluoronitrobenzene derivatives in Suzuki-Miyaura coupling reactions. J Org Chem. 2010;75:5860-5866.

${ }^{234}$ Bahmanyar S, Borer BC, Kim YM, Kurtz DM, Yu S. Proximity effects in the palladiumcatalyzed substitution of aryl fluorides. Org Lett. 2005;7:1011-1014.

${ }^{235}$ Yu D, Shen Q, Lu L. Selective palladium-catalyzed C-F activation/carbon-carbon bond formation of polyfluoroaryl oxazolines. J Org Chem. 2012; 77:1798-1804.

${ }^{236} \mathrm{Yu} \mathrm{D}, \mathrm{Lu} \mathrm{L}$, Shen Q. Palladium-catalyzed coupling of polyfluorinated arenes with heteroarenes via C-F/C-H activation. Org Lett. 2013;15:940-943. 
${ }^{237}$ Braun T, Izundu J, Steffen A, Neumann B, Stammler HG. Reactivity of a palladium fluoro complex towards silanes and $\mathrm{Bu}_{3} \mathrm{SnCH}=\mathrm{CH}_{2}$ : Catalytic derivatisation of pentafluoropyridine based on carbon-fluorine bond activation reactions. Dalton Trans. 2006;5118-5123.

${ }^{238}$ Breyer D, Braun T, Kläring P. Synthesis and reactivity of the fluoro complex trans- $[\mathrm{Pd}(\mathrm{F})(4-$ $\left.\left.\mathrm{C}_{5} \mathrm{NF}_{4}\right)\left(\mathrm{Pr}_{2} \mathrm{PCH}_{2} \mathrm{CH}_{2} \mathrm{OCH}_{3}\right)_{2}\right]$ : $\mathrm{C}-\mathrm{F}$ bond formation and catalytic $\mathrm{C}-\mathrm{F}$ bond activation reactions. Organometallics 2012;31:1417-1424.

${ }^{239}$ Dankwardt JW. Transition metal catalyzed cross-coupling of aryl Grignard reagents with aryl fluorides via Pd- or Ni-activation of the $\mathrm{C}-\mathrm{F}$ bond: an efficient synthesis of unsymmetrical biaryls - application of microwave technology in ligand and catalyst screening. J Organomet Chem. 2005;690:932-938.

${ }^{240}$ Saeki T, Takashima Y, Tamao K. Nickel- and palladium-catalyzed cross-coupling reaction of polyfluorinated arenes and alkenes with Grignard reagents. Synlett. 2005;1771-1774.

241 (a) Manabe K, Ishikawa S. Ortho-selective cross-coupling of fluorobenzenes with Grignard reagents: acceleration by electron-donating ortho-directing groups. Synthesis 2008;26452649. (b) Ishikawa S, Manabe K. Synthesis of substituted monohalobenzenes via orthoselective cross- coupling of dihalobenzenes with electron-donating ortho-directing groups. Synthesis 2008;3180-3182.

${ }^{242}$ Sun L, Rong M, Kong D, Bai Z, Yuan Y, Weng Z. Synthesis of polyfluorinated aryl ethers via ligand-free palladium- catalyzed $\mathrm{C}-\mathrm{F}$ activation of pentafluorobenzene. J Fluorine chem. 2013; 150:117-123.

${ }^{243}$ Braun T, Rothfeld S, Schorlemer V, Stammler A, Stammler HG. Palladium mediated activation of a $\mathrm{C}-\mathrm{F}$ bond in pentafluoropyridine: synthesis, structure and reactivity of a pyridyloxy complex. Inorg Chem Commun. 2003;6:752-755.

${ }^{244}$ Scharf A, Goldberg I, Vigalok A. Palladium-assisted room-temperature nucleophilic substitution of an unactivated aryl fluoride. Organometallics 2012;31:1275-1277.

${ }^{245}$ Ohashi M, Kambara T, Hatanaka T, Saijo H, Doi R, Ogoshi S. Palladium-catalyzed coupling reactions of tetrafluoroethylene with arylzinc compounds. J Am Chem Soc. 2011;133:32563259.

${ }^{246}$ Ohashi M, Shibata M, Saijo H, Kambara T, Ogoshi S. Carbon-fluorine bond activation of tetrafuoroethylene on palladium( 0 ) and nickel(0): heat or lewis acidic additive promoted oxidative addition. Organometallics 2013;32:3631-3639.

${ }^{247}$ Ohashi M, Saijo H, Shibata M, Ogoshi S. Palladium-catalyzed base-free Suzuki-Miyaura coupling reactions of fluorinated alkenes and arenes via a palladium fluoride key intermediate. Eur J Org Chem. 2013;443-447.

${ }^{248}$ Heitz W, Knebelkamp A. Makromol Chem Rapid Commun. 1991;12:69-75.

${ }^{249}$ Yokota M, Fujita D, Ichikawa J. Org. Lett. 2007;9:4639-4642.

${ }^{250}$ (a) Sakoda K, Mihara J, Ichikawa J. Chem Commun. 2005;4684-4686. (b) Ichikawa J, Sakoda K, Mihara J, Ito N. Heck-type 5-endo-trig cyclizations promoted by vinylic fluorines: Ring-fluorinated indene and $3 \mathrm{H}$-pyrrole syntheses from 1,1-difluoro-1-alkenes. $J$ Fluorine chem. 2006;127:489-504.

${ }^{251}$ Ichikawa J, Nadano R, Ito, N. 5-endo Heck type cclization of 2-(trifluoromethyl)allyl ketone oximes: synthesis of 4-difluoromethylene-substituted 1-pyrrolines. Chem Commun. 2006;4425-4427.

${ }^{252}$ Hazari A, Gouverneur V, Brown JM. Palladium-catalyzed substitution of allylic fluorides Angew Chem Int Ed. 2009;48:1296-1299.

253 (a) Pigeon X, Bergeron M, Barabé F, Dubé P, Frost HN, Paquin JF. Activation of allylic C-F bonds: Palladium-catalyzed allylic amination of 3,3-difluoropropenes. Angew Chem Int Ed. 2010;49:1123-1127. (b) Paquin JF. Synthesis of monofluoroalkenes via the activation of allylic C-F bonds: A novel route to $\beta$-aminofluoroalkenes using Pd-catalyzed allylic amination reactions of 3,3-difluoropropenes. Synlett. 2011;289-293.

${ }^{254}$ Kuehnel MF, Lentz D, Braun T. Synthesis of fluorinated building blocks by transitionmetal-mediated hydrodefluorination reactions. Angew. Chem. Int. Ed. 2013;52:3328-3348. 
${ }^{255}$ Hudlicky M. Catalytic hydrogenolysis of carbon-fluorine bonds: $\pi$-bond participation mechanism. J. Fluor Chem. 1989;44:345-359.

${ }^{256}$ Ukisu Y, Miyadera T. Hydrogen-transfer hydrodehalogenation of aromatic halides with alcohols in tiaepresence of noble metal catalysts. J Mol Catal A: Chem. 1997;125:135-142.

${ }^{257}$ Yang H, Gao H, Angelici RJ. Hydrodefluorination of fluorobenzene and 1,2-difluorobenzene under mild conditions over rhodium pyridylphosphine and bipyridyl complexes tethered on a silica-supported palladium catalyst. Organometallics 1999;18:2285-2287.

${ }^{258}$ Sabater S, Mata JA, Peris E. Hydrodefluorination of carbon-fluorine bonds by the synergistic action of a ruthenium-palladium catalyst. Nature Commun. 2013;4:2553. DOI: 10.1038/ncomms3553.

${ }^{259}$ Breyer D, Braun T, Pender A. Isolation and reactivity of palladium hydrido complexes: intermediates in the hydrodefluorination of pentfluoropyridine. Dalton Trans. 2010;39:75137520 .

${ }^{260}$ Chen Z, He CY, Yin Z, Chen L, He Y, Zhang X. Palladium-catalyzed ortho-selective C-F activation of polyfluoroarenes with triethylsilane: a facile access to partially fluorinated aromatics. Angew Chem Int Ed. 2013;52:5813-5817.

${ }^{261}$ Narumi T, Tomita K, Inokuchi E et al. Facile synthesis of fluoroalkenes by palladiumcatalyzed reductive defluorination of allylic gem-difluorides. Org Lett. 2007;9:3465-3468.

${ }^{262}$ Hamashima Y, Yagi K, Takano H, Tamás L, Sodeoka M. An efficient enantioselective fluorination of various $\beta$-ketoesters catalyzed by chiral palladium complexes. J Am Chem Soc. 2002;124:14530-14531.

${ }^{263}$ Hamashima Y, Suzuki T, Takano H et al. Highly enantioselective fluorination reactions of $\beta$ ketoesters and $\beta$-ketophosphonates catalyzed by chiral palladium complexes. Tetrahedron 2006;62:7168-7179.

${ }^{264}$ Hamashima Y, Takano H, Hotta D, Sodeoka M. Immobilization and reuse of Pd complexes in ionic liquid: efficient catalytic asymmetric fluorination and Michael reactions with $\beta$ ketoesters. Org Lett. 2003;18:3225-3228.

${ }^{265} \mathrm{Kim}$ SM, Kim HR, Kim DY. Catalytic enantioselective fluorination and amination of $\beta$-keto phosphonates catalyzed by chiral palladium complexes. Org Lett. 2005;7:2309-2311

${ }^{266} \mathrm{Kim}$ HR, Kim DY. Catalytic enantioselective fluorination of $\alpha$-cyano acetates catalyzed by chiral palladium complexes. Tetrahedron Lett. 2005;46:3115-3117.

${ }^{267}$ (a) Paull DH, Scerba MT, Alden-Danforth E, Widger LR, Lectka T. Catalytic, asymmetric $\alpha-$ fluorination of acid chlorides: dual metal-ketene enolate activation. J Am Chem Soc. 2008;130:17260-17261. (b) Erb J, Paull DH, Dudding T, Belding L, Lectka T. From bifunctional to trifunctional (tricomponent nucleophile-transition metal-lewis acid) catalysis: the catalytic, enantioselective $\alpha$-fluorination of acid chlorides. J Am Chem Soc. 2011;133:7536-7546.

${ }^{268}$ Hull KL, Anani WQ, Sanford MS. Palladium-catalyzed fluorination of carbon-hydrogen bonds. J Am Chem Soc. 2006;128:7134-7135.

${ }^{269}$ Wang X, Mei TS, Yu JQ. Versatile $\mathrm{Pd}(\mathrm{OTf})_{2} \cdot 2 \mathrm{H}_{2} \mathrm{O}$-catalyzed ortho-fluorination using NMP as a promoter. J Am Chem Soc. 2009;131:7520-7521.

${ }^{270}$ Chan KSL, Wasa M, Wang X, Yu JQ. Palladium(II)-catalyzed selective monofluorination of benzoic acids using a practical auxiliary : a weak-coordination approach. Angew Chem Int Ed. 2011;50:9081-9084

${ }^{271}$ Furuya T, Kaiser HM, Ritter T. Palladium-Mediated Fluorination of Arylboronic Acids. Angew Chem Int Ed. 2008;47:5993-5006.

${ }^{272}$ Furuya T, Benitez D, Tkatchouk E, et al. Mechanism of C-F reductive elimination from palladium(IV) fluorides. J Am Chem Soc. 2010;132:3793-3807.

${ }^{273}$ Mazzotti AR, Campbell MG, Tang P, Murphy JM, Ritter T. Palladium(III)-catalyzed fluorination of arylboronic acid derivatives. J Am Chem Soc. 2013;135:14012-14015.

${ }^{274}$ Peng H, Liu G. Palladium-Catalyzed Tandem Fluorination and Cyclization of Enynes. Org Lett. 2011; 13:772-775. 
${ }^{275}$ Qui S, Xu T, Zhou J, Guo Y, Liu G. Palladium-catalyzed intermolecular aminofluorination of styrenes. J Am Chem Soc. 2010;132:2856-2857.

${ }^{276} \mathrm{Wu}$ T, Yin G, Liu G. Palladium-catalyzed intramolecular aminofluorination of unactivated alkenes. J Am Chem Soc. 2009;131:16354-16355.

${ }^{277}$ Engle KM, Mei TS, Wang X, Yu JQ. Bystanding $\mathrm{F}^{+}$oxidants enable selective reductive elimination from high-valent metal centers in catalysis. Angew Chem Int Ed. 2011;50:14781491.

${ }^{278}$ See for example: Hartwig JF. Evolution of a fourth generation catalyst for the amination and thioetherification of aryl halides. Acc Chem Res. 2008;41:1534-1544 and references therein.

${ }^{279}$ Watson DA, Su M, Teverovskiy G et al. Formation of ArF from LPdAr(F):

Catalytic conversion of aryl triflates to aryl fluorides. Science 2009;325:1661-1664.

${ }^{280}$ Lee HG, Milner PJ, Buchwald SL. An improved catalyst system for the Pd-catalyzed fluorination of (hetero)aryl triflates. Org Lett. 2013;15:5602-5605.

${ }^{281}$ Noël T, Maimone TJ, Buchwald SL. Accelerating palladium-catalyzed C-F bond formation: use of a microflow packed-bed reactor. Angew Chem Int Ed. 2011;50:8900-8903.

${ }^{282}$ Maimone TJ, Milner PJ, Kinzel T, Zhang Y, Takase MK, Buchwald SL. Evidence for in situ catalyst modification during the pd-catalyzed conversion of aryl triflates to aryl fluorides. $J$. Am. Chem. Soc. 2011;133:18106-18109.

${ }^{283}$ Lee HG, Milner PJ, Buchwald SL. Pd-catalyzed nucleophilic fluorination of aryl bromides. $J$. Am. Chem. Soc. 2014; doi.org/10.1021/ja5009739.

${ }^{284}$ Lee E, Kamlet AS, Powers DC et al. A fluoride-derived electrophilic late-stage fluorination reagent for PET imaging. Science 2011;334:639-642.

${ }^{285}$ Sakakura T, Chaisupakitsin M, Hayashi T, Tanaka M. Efficient acid fluoride synthesis via carbonylation of organic halides. J Organomet Chem. 1987;334:205-2011.

${ }^{286}$ Hollingworth C, Hazari A, Hopkinson MN et al. Palladium catalyzed allylic fluorination. Angew Chem Int Ed. 2011;50:2613-2617.

${ }^{287}$ Katcher MH, Doyle AG. Palladium-catalyzed asymmetric synthesis of allylic fluorides. $J$ Am Chem Soc. 2010;132:17402-17404.

${ }^{288}$ Katcher MH, Sha A, Doyle AG Palladium-catalyzed regio- and enantioselective fluorination of acyclic allylic halides. J Am Chem Soc. 2011;133:15902-15905.

${ }^{289}$ Fraser SL, Antipin MY, Khroustalyov VN, Grushin VV. Molecular fluoro palladium complexes. J Am Chem Soc. 1997;119:4769-4770.

${ }^{290}$ Pilon MC, Grushin VV. Synthesis and characterization of organopalladium complexes containing a fluoro ligand. Organometallics 1998;17:1774-1781.

${ }^{291}$ Grushin VV, Palladium fluoride complexes: one more step toward metal-mediated C-F bond formation. Chem Eur J. 2002;8:1006-1014.

${ }^{292}$ a) Yahav A, Goldberg I, Vigalok, A. Synthesis of the elusive $\left(\mathrm{R}_{3} \mathrm{P}\right)_{2} \mathrm{MF}_{2}(\mathrm{M}=\mathrm{Pd}, \mathrm{Pt})$ complexes. J Am Chem Soc. 2003;125:13634-13635 b) Kaspi AW, Yahav-Levi, Goldberg I, Vigalok A. Xenon difluoride induced aryl iodide reductive elimination: a simple access to difluoropalladium(II) complexes. Inorg Chem. 2008;47:5-7.

${ }^{293}$ Braun T, Steffen A, Schorlemer V, Neumann B, Stammler HG. Routes to unique palladium A-frame complexes with a bridging fluoro-ling and. Dalton Trans. 2005;3331-3336.

${ }^{294}$ Grushin VV, Marshall WJ. Is fluoride bonded to two Pd acceptors still basic? Three $\mathrm{CH}_{2} \mathrm{Cl}_{2}$ molecules encapsulating a $\mathrm{Pd}_{2}(\mu-\mathrm{F})_{2}$ square and new implications for catalysis. Angew Chem Int Ed. 2002;41:4476-4479.

${ }^{295}$ Grushin VV, Marshall WJ. Ar-F reductive elimination from palladium(II) revisited. Organometallics 2007;26:4997-5002.

${ }^{296}$ Yandulov DV, Tran NT. Aryl-fluoride reductive elimination from Pd(II): Feasibility assessment from theory and experiment. J Am Chem Soc. 2007;129:1342-1358.

${ }^{297}$ Flemming JP, Pilon MC, Borbulevitch OY, Antipin MY, Grushin VV. The trans influence of $\mathrm{F}, \mathrm{CI}, \mathrm{Br}$ and I ligands in a series of square-planar Pd(II) complexes. Relative affinities of halide anions for the metal centre in trans- $\left[\left(\mathrm{Ph}_{3} \mathrm{P}\right)_{2} \mathrm{Pd}(\mathrm{Ph}) \mathrm{X}\right]$. Inorg Chim Acta 1998;280:8798. 
${ }^{298}$ Martínez-Prieto LM, Melero C, del Río D, Palma P, Cámpora J, Álvarez E. Synthesis and reactivity of nickel and palladium fluoride complexes with PCP pincer ligands. NMR-based assessment of electron donating properties of fluoride and other monoanionic ligands. Organometallics 2012; 31:1425-1438.

${ }^{299}$ Marshall WJ, Thorn DL, Grushin VV. Single-Crystal X-ray and solution ${ }^{13}$ C NMR study of fluoro(p-nitrophenyl)bis(triphenylphosphine)palladium(II). are there effects of throughconjugation? Organometallics 1998; 17:5427-5430.

${ }^{300}$ Grushin VV. Generation of a "naked" fluoride ions in unprecedentedly high concentrations from a fluoropalladium complex. Angew Chem Int Ed. 1998;37:994-996.

${ }^{301}$ Roe DC, Marshall WJ, Davidson F, Soper PD, Grushin VV. Structure and solution synamics of $\left[\left(\mathrm{Ph}_{3} \mathrm{P}\right)_{2} \mathrm{Pd}(\mathrm{Ph})(\mathrm{FHF})\right]$. Organometallics 2000;19:4575-4582.

${ }^{302}$ Furuya T, Ritter T. Carbon-fluorine reductive elimination from a high-valent palladium fluoride. J Am Chem Soc. 2008;130:10060-10061.

${ }^{303}$ Ball ND, Sanford MS. Synthesis and reactivity of a mono- $\sigma$-aryl palladium(IV) fluoride complex. J Am Chem Soc. 2009;131:3796-3797.

${ }^{304}$ Bosque R, Clot E, Fantacci S, et al. Inertness of the aryl-F bond toward oxidative addition to osmium and rhodium complexes: thermodynamic or kinetic origin? J Am Chem Soc. 1998;120:12634-12640.

${ }^{305}$ Braun T, Cronin L, Higgitt CL, McGrady JE, Perutz RN, Reinhold M. Coordination and oxidative addition of octafluoronaphthalene at a nickel centre: Isolation of an intermediate in C-F bond activation. New J Chem. 2001;25:19-21.

${ }^{306}$ Johnson SA, Taylor ET, Cruise SJ. A Combined experimental and computational study of unexpected C-F Bond activation intermediates and selectivity in the reaction of pentafluorobenzene with a $\left(\mathrm{PEt}_{3}\right)_{2} \mathrm{Ni}$ synthon. Organometallics 2009;28:3842-3855.

${ }^{307}$ Schaub T, Fischer P, Steffen A, Braun T, Radius U, Mix A. C-F Activation of fluorinated arenes using NHC-stabilized nickel(0) complexes: Selectivity and mechanistic investigations. J Am Chem Soc. 2008;130:9304-9317

${ }^{308}$ Jiao E, Xia F, Zhu, H. Density functional study for the C-F bond activation of the reaction of $\left[\mathrm{Pt}\left(\mathrm{PCy}_{3}\right)_{2}\right]$ with $\mathrm{C}_{6} \mathrm{~F}_{6}$. Comp Theor Chem. 2011;965:92-100.

${ }^{309}$ Macgregor SA, Roe DC, Marshall WJ, Bloch KM, Bakhmutov VI, Grushin VV. The F/Ph rearrangement reaction of $\left[\left(\mathrm{Ph}_{3} \mathrm{P}\right)_{3} \mathrm{RhF}\right]$, the fluoride congener of Wilkinson's catalyst. $J \mathrm{Am}$ Chem Soc. 2005; 127:15304-15321.

${ }^{310}$ Cronin L, Higgitt CL, Karch R, Perutz RN. Rapid intermolecular carbon-fluorine bond activation of pentafluoropyridine at nickel(0): comparative reactivity of fluorinated arene and fluorinated pyridine derivatives. Organometallics 1997;16:4920-4928.

${ }^{311}$ Jasim NA, Perutz RN, Whitwood AC, et al. Contrasting reactivity of fluoropyridines at palladium and platinum: C-F oxidative addition at palladium, P-C and C-F activation at alatinum. Organometallics 2004;23:6140-6149.

${ }^{312}$ Clot E, Eisenstein O, Jasim N, MacGregor SA, McGrady JE, Perutz RN. C-F and C-H bond activation of fluorobenzenes and fluoropyridines at transition metal centers: how fluorine tips the scales. Acc Chem Res. 2011;44:338-348.

${ }^{313}$ Nova A, Erhardt S, Jasim NA, et al. Competing C-F activation pathways in the reaction of $\mathrm{Pt}(0)$ with fluoropyridines: Phosphine-assistance versus oxidative addition. J Am Chem Soc. 2008;130:15499-15511.

${ }^{314}$ Jakt M, Johannissen L, Rzepa HS, Widdowson DA, Wilhelm R. A computational study of the mechanism of palladium insertion into alkynyl and aryl carbon-fluorine bonds. J Chem Soc Perkin Trans. 2 2002;576-581.

${ }^{315}$ Doyle, G. The reaction of formyl fluoride with transition metal complexes J Organomet Chem. 1982;224:355-362.

${ }^{316}$ Kurosawa H, Ogoshi S, Kawasaki Y, Murai S, Miyoshi M, Ikeda I. Novel dependency of stereochemistry upon metal, ligand, and solvent in oxidative addition of allylic chloride to Pd(0) and Pt(0) complexes. J Am Chem Soc. 1990;112:2813-2814. 
${ }^{317}$ Granberg KL, Bäckvall JE. Isomerization of ( $\pi$-allyl)palladiumcomplexes via nucleophilic displacement by palladium(0). A common mechanism in palladium(0)-catalyzed allylic substitution. J Am Chem Soc. 1992;114:6858-6863.

${ }^{318}$ Zhao H, Ariafard A, Lin Z. $\beta$-Heteroatom versus $\beta$-hydrogen elimination: A theoretical study. Organometallics 2006;25:812-819.

${ }^{319}$ Brothers PJ, Roper WR. Transition-metal dihalocarbene complexes. Chem Rev. 1988;88:1293.

${ }^{320}$ Hughes RP. Conversion of carbon-fluorine bonds $\alpha$ to transition metal centers to carbonhydrogen, carbon-carbon, and carbon-heteroatom bonds. Eur J Inorg Chem. 2009:45914606.

${ }^{321}$ Usón R, Forniés J, Espinet $\mathrm{P}$ et al. $\mathrm{C}\left(\mathrm{C}_{6} \mathrm{~F}_{4} \mathrm{PPh}_{2}-\mathrm{O}\right) \mathrm{NMe}$ a C,P-Chelate and $\mathrm{C}, \mathrm{N}$-bridging ligand. Crystal structure of $\left[\left\{\mathrm{Pd}\left[\mathrm{C}\left(\mathrm{C}_{6} \mathrm{~F}_{4} \mathrm{PPh}_{2}-\mathrm{O}\right) \mathrm{N}(\mathrm{Me})\right] \mathrm{Cl}\right\}_{2}\right] 0.88(4)-\mathrm{CH}_{2} \mathrm{Cl}_{2}$. J Organomet Chem. 1985;282:C35-C38.

${ }^{322}$ Grushin VV. Thermal stability, decomposition paths, and $\mathrm{Ph} / \mathrm{Ph}$ exchange reactions of $\left[\left(\mathrm{Ph}_{3} \mathrm{P}\right)_{2} \mathrm{Pd}(\mathrm{Ph}) \mathrm{X}\right]\left(\mathrm{X}=\mathrm{I}, \mathrm{Br}, \mathrm{Cl}, \mathrm{F}\right.$, and $\left.\mathrm{HF}_{2}\right)$. Organometallics 2000;19:1888-1900.

${ }^{323}$ Marshall WJ, Grushin VV. Palladium(II) and palladium(0) complexes of BINAP(O) (2(diphenylphosphino)-2'-(diphenylphosphinyl)-1,1'-binaphthyl). Organometallics 2003;22:555-562.

${ }^{324}$ Racowski J.M, Gary JB, Sanford MS. Carbon(sp3) fluorine bond-forming reductive elimination from palladium(IV) complexes. Angew Chem Int Ed. 2012;51:3414-3417.

${ }^{325}$ Pérez-Temprano MH, Racowski JM, Kampf JW, Sanford MS. Competition between $\mathrm{sp}^{3}-\mathrm{C}-\mathrm{N}$ vs sp ${ }^{3}-\mathrm{C}-\mathrm{F}$ reductive elimination from $\mathrm{Pd}^{\mathrm{IV}}$ complexes $J$ Am Chem Soc. 2014;136:4097-4100. 